Portland State University

PDXScholar

$1-1-1987$

\title{
Segment Congruence Analysis: An Information Theoretic Approach
}

Jamshid Hosseini-Chaleshtari

Portland State University

Follow this and additional works at: https://pdxscholar.library.pdx.edu/open_access_etds Let us know how access to this document benefits you.

Recommended Citation

Hosseini-Chaleshtari, Jamshid, "Segment Congruence Analysis: An Information Theoretic Approach" (1987). Dissertations and Theses. Paper 797.

https://doi.org/10.15760/etd.797

This Dissertation is brought to you for free and open access. It has been accepted for inclusion in Dissertations and Theses by an authorized administrator of PDXScholar. Please contact us if we can make this document more accessible: pdxscholar@pdx.edu. 
SEGMENT CONGRUENCE ANALYSIS:

AN INFORMATION THEORFTIC APPROACH

by

JAMSHID HOSSEINI-CHALESHTARI

A dissertation submitted in partial fulfillment of the requirements for the degree of

DOCTOR OF PHILOSOPHY
in

SYSTEMS SCIENCE

Portland State University

(C) 1987 
TO THE OFFICE OF GRADUATE STUDIES AND RESEARCH:

The members of the committee approve the dissertation of Jamshid Hosseini-Chaleshtari presented June 29, 1987.

R.bbert R. Harmon, Chairman

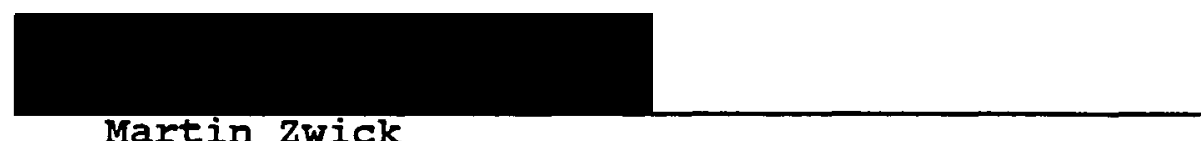

Martin 2wick

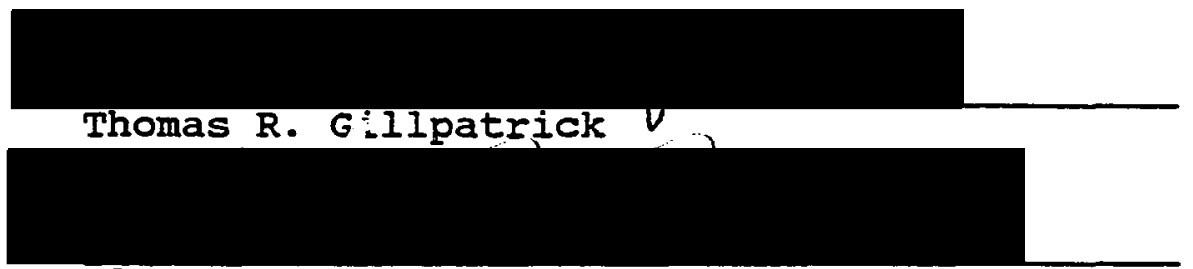

Alan R. Raedels
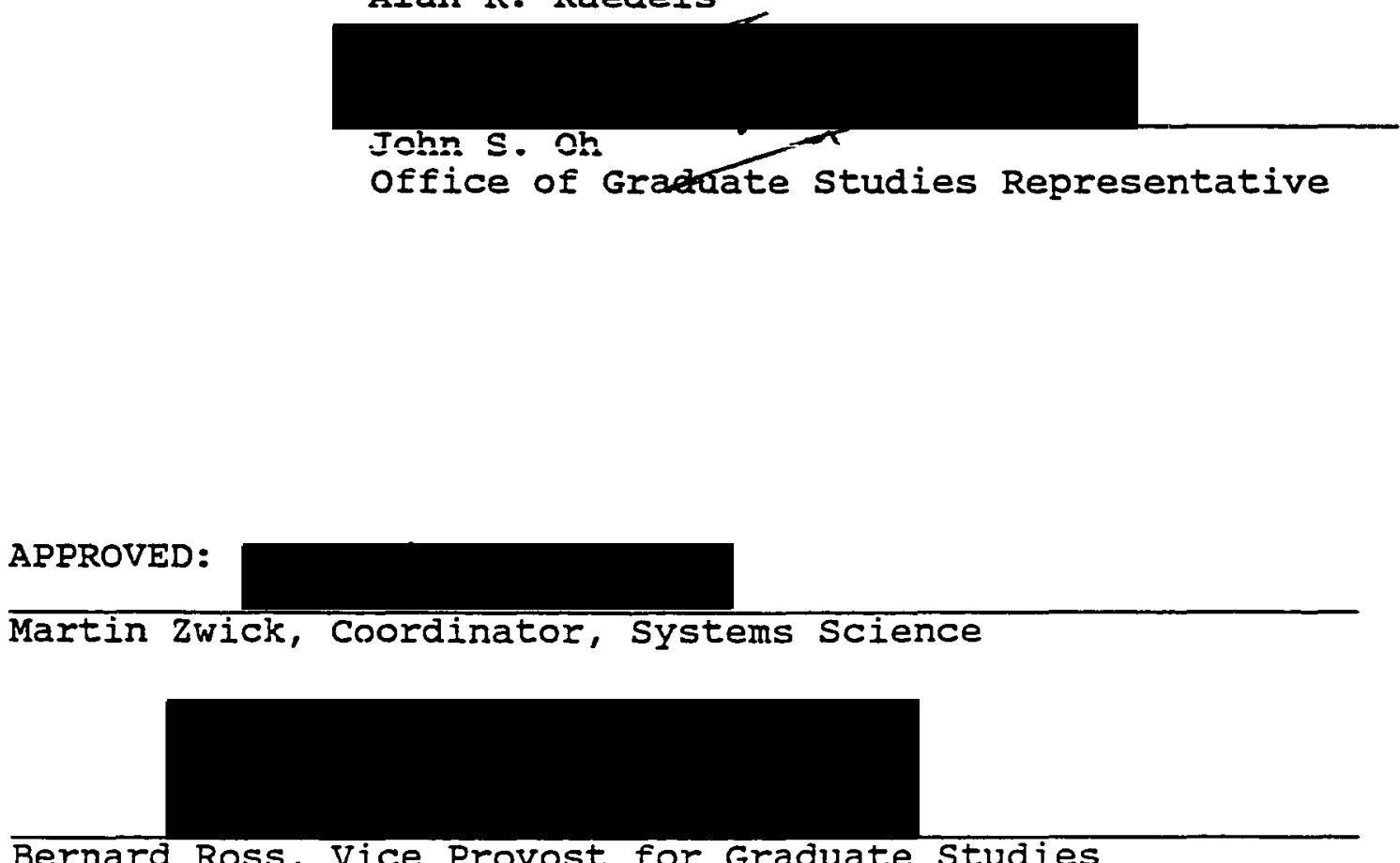

Bernard Ross, Vice Provost for Graduate Studies 
To my father, Khodayar, and my mother, Tooba, whose unconditional love has been a guiding force in my life and an inspiration to me as a parent.

To my lovely wife, Pamela, whose patience and loving support provided the peace of mind I needed to undertake this project.

And to my children, Nema and Arman, who give meaning and purpose to my life. 


\section{ACKNOWLEDGEMENTS}

I wish to express my sincere gratitude to the many individuals who provided assistance and encouragement to me throughout my studies. In particular, I am immeasurably indebted to Dr. Robert Harmon whose support I could rely on and whose guidance kept me on track. He was always willing to listen and offer well placed and practical advice. I have been most fortunate to know him as a teacher, a friend, and a colleague.

Words cannot express my respect and gratitude for Dr. Martin Zwick whose quest for excellence and perfection has had a profound effect on my academic and personal life. He introduced me to the general systems methodology area and, during the past several. years, he has helped me to develop scientific and critical thinking. I am proud and honored to have had the benefit of knowing and working with such a true scholar.

My grateful appreciation also extends to the other members of my dissertation committee, Drs. Thomas Gilipatrick, Alan Raedels, and John Oh, for their generous help and kindly advice. Their discussions and comments 
regarding the nature and direction of this work were both stimulating and constructive.

other colleagues and friends also assisted me in many ways. I am especially thankful to my friend Muhannad Khawaja who was an excellent listener and facilitated the acquisition of the data I used for this study. To that end, I am thankful to Dr. Gill Peach and Ms. Karen Schoch of Pacific Power and Light Company and Mike Austin and Erick Westman of Bonneville Power Administration who were instrumental in the process.

I am also indebte $\mathbf{a}$ to Mr. Wess Brenner, Ms. An Hotan, and Mr. Dave Sato of the Academic Computing at Portland State University for their technical support and good humor. Ms. Dawn Kuenle of the System Science Department, Ms. Maureen Eldred and Berni Pilip of the Office of Graduate Studies and, Ms. Sally Lopez all helped facilitate the operational aspects of my dissertation for which I am grateful.

Finally, my beloved family, my lovely wife, Pamela, my dearest sons Nema and Arman, my brother Behzad and my sister Maryam, deserve immeasurable gratitude for keeping me sane throughout this work. Not many people are fortunate enough to have so many individuals on their side and so much encouragement as I had. 
AN ABSTRACT OF THE DISSERTATION OF Jamshid HosseiniChaleshtari for the Doctor of Philosophy in Systems Science presented June 29, 1987.

Title: Segment Congruence Analysis: An Information Theoretic Approach

APPROVED BY MEMBERS OF THE DISSERTATION COMMITTEE:

Robert R. Harmon, Chairman

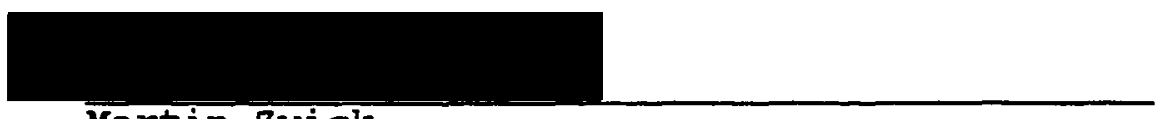

Martin $\overline{\text { Zwick }}$

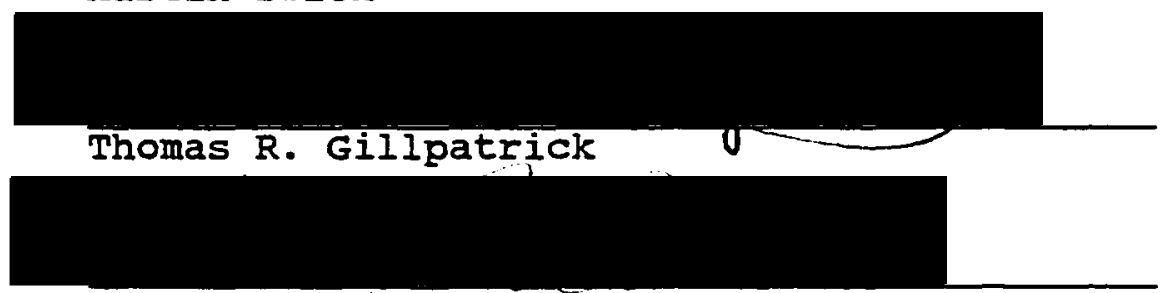

Alan R. Raedels

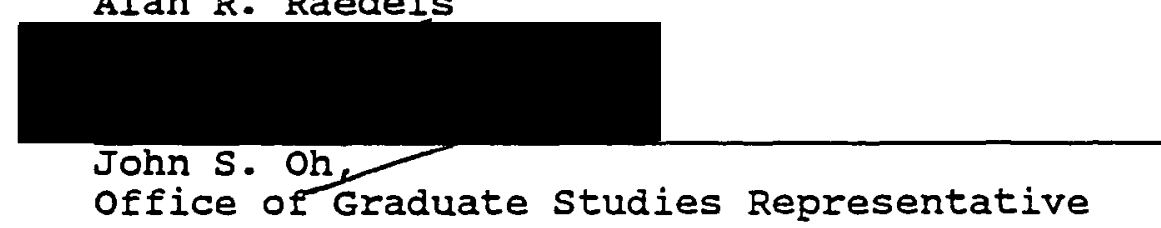

During the past three decades, marketers have developed various methods to partition the total market into subgroups of consumers whose responses to certain marketing strategies are homogeneous within the subgroups and heterogeneous across them. Usually, this analysis begins by identifying a segmentation variable (a priori and/or by 
clustering) which characterizes consumer behavior. A particular class of this variable is identified as the target segment for which a marking strategy is to be developed. This target segment is then analyzed in terms of descriptor variables.

When there are several possible segmentation variables, marketers must investigate the ramifications of their potential interactions. These include their mutual association, the identification of the best (the distinguished) segmentation variable and its predictability by a set of descriptor variables, and the structure of the multivariate system(s) obtained from the segmentation and descriptor variables. This procedure has been defined as segment congruence analysis ( $\underline{S C A}$ ) (Green and Carmone 1977). Traditionally, this has been done using general loglinear and logit models. This study utilizes the information theoretic approach, as well as the loglinear/logit approach, to address a variety of research questions in segment congruence analysis. It is shown that the information theoretic approach expands the scope of SCA and offers some advantages over traditional methods.

Data obtained from a survey conducted by the Bonneville Power Administration and Northwest utilities (PNWRES, the 1983 Pacific Northwest Residential Survey) is used to demonstrate the efficacy of the information theoretic and the log-Iinear/logit approaches and compare 
these two methods. The survey was designed to obtain information on energy consumption habits, attitudes toward selected energy issues, and the conservation measures utilized by the residents in the Pacific Northwest

The analyses are performed in two distinct phases. Phase I includes assessment of mutual association among segmentation variables and four methods (based on different information theoretic functions) for identifying candidates for the distinguished variable. Phase II addresses the selection and analysis of the distinguished variable. This variable is selected either a priori or by assessment of its predictability from (segmentation or exogenous) descriptor variables. The relations between the distinguished variable and the descriptor variables are further analyzed by examining the predictability issue in greater detail and by evaluating structural models of the multivariate systems. SPSS $^{X}(1986)$ is used to 1) combine clusters of conceptually related variables in the original survey data into aggregate variables; 2) perform general log-linear, hierarchical log-linear, and logit analyses; and 3) produce input files for the information theory program (a FORTRAN 77 program) .

The methodological conclusions of this study are that the information theoretic and log-linear methods have deep similarities. Therefore, it is possible to utilize the readily available information theoretic measures in 
developing log-linear models. In this study, several new log-linear models, which have not been used in the past, are developed based on their information theoretic counterparts. PNWRES analyses produced intuitively plausible results. In Phase I, energy related awareness, behavior, perceptions, attitudes, and electricity consumption were identified as candidate segmentation variables. In Phase II, using exogenous descriptor variables, electricity consumption was selected as the distinguished variable. The analysis of this variable indicated that the demographic factors, type of dwelling, and geoclimatic environment are among the most important determinants of electricity consumption. 
TABLE OF CONTENTS

PAGE

ACKNOWLEDGEMENTS...................... iv

TABLE OF CONTENTS....................... vi

IIST OF TABLES........................ $\mathrm{x}$

LIST OF FIGURES.......................

CHAPTER

I INTRODUCTION..................... I

objectives of the study............ 4

Motivation for the study........... 11

II REVIEW OF THE IITERATURE.............. 14

An Overview of Market Segmentation..... 15

Segment Congruence Analysis......... 19

The Role of Contingency Table Analysis

The Log-Iinear/Logit Approach........

Relevant Applications of the General Log-Linear Model

Relevant Applications of the Logit Model 
The Information Theoretic Approach.....

Some Important Properties of Information Theory

Information Theoretic Definitions of a leading Part

statistical Significance of Informational Measures Information Theory as a Tool for Segment Congruence Analysis

The General Probability and Chi-square Approach.................... 50

summary..................... 53

III RESEARCH QUESTIONS, METHODOLOGY, AND

PROCEDURES.................... 56

Research Questions............... 56

Phase I: Analysis of the overlap

Phase II: Identification and Analysis of the Distinguished Variable

Methodology.....................

Information Theoretic Methodology

Computer Program

The General Log-Linear/Logit Approach

Sumnary of the Methodology

The Data and Procedures............

Description of the study

Selection and Aggregation of

Variables 
Analysis of the segmentation

Variables.

Existence and Degree of Mutual Association Among Segmentation Variables

Identification of candidates for the Distinguished Variables

Determination and Analysis of the

Distinguished Segmentation Variable

Using the other segmentation

Variables as the Descriptor

Variables...................

Electricity Consumption as the Distinguished Segmentation Variable (A Priori)

Analytical Identification of the Distinguished segmentation Variable

Determination and Analysis of the Distinguished Segmentation Variable Using Exogenous Descriptor

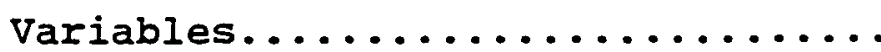

Summary of Analysis.............. 176

$\mathrm{V}$ CONCLUSIONS AND FUTURE DIRECTIONS........... 181

Findings of the study............. 181 
Contributions of the study............

Contribution to segment congruence Analysis

Contribution to Market Segmentation Contribution to Log-Iinear Modeling Contribution to Information Theoretic Modeling

Limitations.................. 196

Directions for Further Research....... 198

BIBLIOGRAPHY........................... 200

APPENDIX I General Log-Linear Models.......... 213

APPENDIX II Entropy and Information Theoretic

Concepts.................. 220

APPENDIX III Pacific Northwest Residential

Consumption Survey (Spring 1983)

- Code Book.................. 229

APPENDIX IV Pacific Northwest Residential

Consumption Survey (1983) - List

of Variables............... 286

APPENDIX $V$ Sample Input/Output for SYSENT, The

Information Theoretic Data

Analysis Package............ 299 
PLEASE NOTE:

Page(s) not included with original material and unavailable from author or university. Filmed as received.

\section{$\mathrm{U} \cdot \mathrm{M} \cdot \mathrm{I}$}


VIII Information Theoretic and Log-Linear Mathematical Expressions Used for Segment Congruence Analysis (Phase

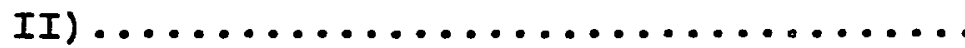

IX Comparison of the Two Approaches to

Segment Congruence Analysis......... 110

$\mathrm{x}$ List of Variables prior to Aggregations... 120

XI Isist of Variables (or Clusters) After

Aggregations.................... 122

XII List of the segmentation Variables....... 127

XIII Assessment of the Mutual Association...... 128

XIV Assessment of the Contribution of Each

Variable to the Mutual Association..... 131

XV Assessment of the Contribution of Each

Variable to the Joint Variability in

the system of segmentation Variables... 136

XVI Assessment of the Contribution of Each

Variable to the Total Uncertainty in

the system of segmentation Variables... 139

XVII Assessment of the Number of significant

Dyadic Relations................. $\quad \cdot 142$

XVIII Predictability of a Particular class vs.

All Other Classes of the Distinguished

variable..................... 
XIX Prioritized Effect of the other

Segmentation Variables on Energy

Consumption ................... 149

$\mathrm{XX}$ Dependency Analysis of the Four-Variable

System of GENATT, ENRATT, PERCEPT, and

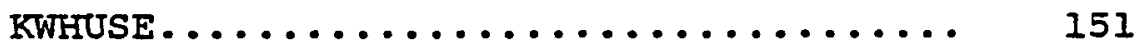

XXI Dependency Analysis of the Six-Variable

System of segmentation Variables.......

XXII Predictability of Candidates for the

Distinguished segmentation Variable....

XXII Predictability of a Particular class vs.

All Other Classes of Energy Related

Behavior......................

XXIV Prioritization of the Effect of the Other

Segmentation Variables on Energy

Related Behavior (BEHAVE)........... 161

XXV Dependency Analysis of the Four-Variable

System of BEHAVE, AWARE, KWHUSE, and

ENRATT.....................

XXVI List of the Exogenous Descriptor

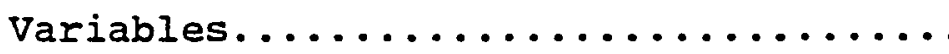

XXVII Predictability of Candidates for the

Distinguished Segmentation Variable

Using Exogenous Descriptor Variables... 
XXVIII Predictability of a Particular Class vs.

All Other Classes of the Distinguished

Variable, (KWHUSE)................ 169

XXIX Prioritized Effect of the Exogenous

Descriptor Variables on Energy

Consumption...................

Xxx Dependency Analysis of the Four-Variable

System of KWHUSE, DEMOG, TYPDWEL, and

CLIMGEO.......................

XXXI Dependency Analysis of the Distinguished

Variable and the Exogenous Descriptor

variables.......................

XXXII Analysis of the Mutual Association Among

Segmentation Variables and

Identification of Candidates for the

Distinguished Segmentation Variable....

XXXIII Analysis Using other Segmentation

Variables as Descriptor Variables

(Electricity Consumption as the

Distinguished Variable (a priori) )....

XXXIV Analysis Using other segmentation

Variables as Descriptor Variables

(Analytical Identification and Analysis

of the Distinguishd Variable)........ 
XXXV Analysis Using Exogenous Descriptor variables...................... 180

XXXVI Significance of the Results of segment

Congruence Analysis to the PNWRES

Study: Phase I.................. 184

XXXVII Significance of the Results of segment

Congruence Analysis to the PNWRES Study

(Phase II: A Priori Distinguished

Variable) .....................

XXXVIII Significance of the Results of segment

Congruence Analysis to the PNWRES study

(Phase II: Other Segmentation Variables

as Descriptor Variables (Analytical))..

XXXIX Significance ot the Results of segment

Congruence Analysis to the PNWRES study

(Phase II: Exogenous Descriptor

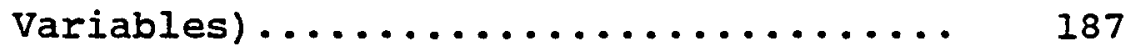

XXXX Actual and Expected cell Frequencies for a $2 \times 2$ Contingency Table............. 


\section{IIST OF FIGURES}

1. A Schematic View of the Total study........ 20

2. Segment Congruence Analysis Process as

Proposed by Green and Carmone (1977)... 22

3. Spatial Representation of Transmission..... 74

4. A System of Four Variables.............. 76

5. Systematic Entropy of a Four-Variable

system....................... 78

6. Determination of Variable $\mathbf{A}$ With the

Knowledge of the Descriptor Variables

$\mathrm{X}, \mathrm{Y}$, and $\mathrm{z} \ldots \ldots \ldots \ldots \ldots \ldots \ldots \ldots \ldots$

7. Prioritization of the contributions of

Variables $X, Y$, and $Z$ to Determine

Variable A.................... 88

8. A Sample Dependency Analysis Diagram..... 92

9. Flow Chart for Selection and Aggregation

of Research Variables..............

10. Flow Chart of the Log-Linear/Logit

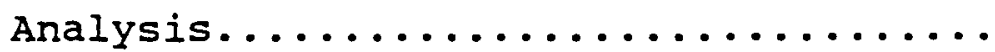

11. Flow chart of the Information Theoretic

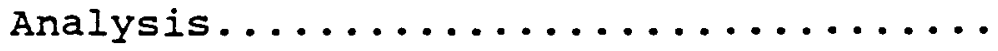


$x v i$

FIGURE

PAGE

12. Condensed Flow Chart of Data Analysis..... 125

13. Graphical Representation of the Dependency Analysis for the Four-Variable system of GENATT, ENRATT, PERCEPT, and KWHUSE.....................

14. Graphical Representation of the Dependency structures for the Six-Variable system of Segmentation Variables........... 156

15. Graphical Representation for the Dependency Structures for the FourVariable system of BEHAVE, AWARE, KWHUSE, and ENRATT..............

16. Graphical Representation of the Dependency Structures for the Four-Variable system of KWHUSE, DEMOG, TYPDWEL, and CLIMGEO. ....................

17. Graphical Representation of the Dependency Structures for the system of Distinguished and Exogenous Variables..................... 
CHAPTER I

\section{INTRODUCTION}

Market segmentation is defined as the grouping of potential customers into subgroups such that their responses to certain market related stimuli (i.e., price, promotion, product, and distribution strategies) are homogeneous within each group and heterogeneous between groups (Lillien and Kotler, 1983). Market segmentation has been an extremely popular subject since late 1940's and early 1950's, and gained prominence through pioneering work by wendell smith (1956) .

The process of market segmentation begins by identifying a variable based on which the market is to be partitioned. Then, different classes of this variable are selected as different market segments. Next, these segments are examined for homogeneity within and heterogeneity across them (e.g., by analyzing variance within and between segments), and stability (e.g., by utilizing the multinomial fixed or variable Markov models) (wind, 1978). Then, the predictability of each segment by a set of descriptor variables is assessed (e.g., through multiple regression 
analysis) (Blattberg and sen, 1976), or its discrimination from other segments is examined (e.g., using multiple discriminant analysis) (Perreault, et al 1979).

In certain market segmentation studies, several variables, instead of one, may be deemed suitable for partitioning the market. In such cases, the primary concern involves the handling of this multivariate problem-- e.g., the identification of a variable to serve as the distinguished segmentation variable, the predictability of this variable given the knowledge of the values, or classes, of other variables, etc.

"Traditionally, researchers have selected some variable or a battery of variables and proceeded from there." (Green 1977, p. 66) This a priori approach either reflects the manager's experience or hunch, or implies the existence of a highly developed body of theory or past research. One disadvantage of this approach is that one's prior convictions can act more as a set of blinders than as guide to further study and understanding. In addition, "regardless of the complexity of reality, human beings find it difficult to classify objects by more than two or three characteristics at a time. If reality requires greater complexity, we are severely constrained by our own conceptual limitations." (Frank et al 1972, p. 122). Strategy considerations should facilitate the choice of segmentation variables. However, even based on strategic 
issues cases can arise in which several candidate bases are plausible choices. What is needed, then, is a method that will:

(1) show how closely related various clusters obtained from alternative bases are, (2) test whether some clusters are independent of the remaining ones, and (3) find the clustering that exhibits the highest contribution to the mutual association among the subset of clusters that evince significant associations in the first place. Furthermore, even if the researcher has settled in advance on a distinguished set of variables to serve as the segmentation base, s/he may be interested in finding out how well the clustering obtained from this distinguished set can be predicted by some function derived from the other clusters (Green, 1977, p. 67)

Analysis of interrelationships among multivariate segmentation problems has been defined as segment congruence analysis (Green and Carmone 1977). The basic premise of this method is that, if mutual association exists among segmentation bases, knowledge of a population unit's membership in one segment may increase or decrease the likelihood of his/her membership in another segment. Segment congruence analysis is useful to the researcher in a number of ways: 1) It enables the elimination of unnecessary segmentation variables. 2) It helps reduce the overlap in marketing mix strategies directed to different segments. 3) It is instrumental in the identification of the "best" (or the "distinguished") segmentation variable. 
OBJECTIVES OF THE STUDY

Not unlike other multivariate problems, segment congruence analysis entails a great deal of complexity. This is due to the fact that, here, several variables are considered simultaneously rather than individually or sequentially. This study investigates two methods of analyzing multivariate problems in the context of segment congruence analysis; the information theoretic approach (i.e., the proposed method) and the log-linear/logit approach (i.e., the traditional method).

The main objectives of this study include: (1) enrichment of segment congruence analysis by introducing numerous relevant questions which have not previously been addressed, (2) introduction and application of information theory as a method to address all of the relevant questions in this area, and (3) comparison of this proposed method with the log-linear/logit models as traditionally used for the purpose of segment congruence analysis.

Data collected through a survey conducted by the Bonneville Power Administration (BPA) in collaboration with the local utilities in the Northwest region (i.e., the Pacific Northwest Residential Energy Consumption Survey, PNWRES) provided the basis for an empirical application of segment congruence analysis. This data was used to 
demonstrate the usefulness of segment congruence analysis (including the newly introduced research questions), the efficacy of the information theoretic approach, and its comparison with the log-linear/logit approach.

The PNWRES study was designed to gain better understanding of energy use habits, attitudes, and conservation activities in the Pacific Northwest region. The BPA decision makers are concerned with energy related issues such as the level of energy usage (i.e., for their energy planning activities) and promotion of conservational programs (particularly, during a period of energy shortage). For both of these purposes, the knowledge of consumers' demographic, socioeconomic, attitudinal, behavioral, and other relevant dimensions, as well as the characteristics of their residence are essential. It is through this knowledge that the energy usage can be predicted, or the success of a conservation oriented program can be estimated prior to its actual implementation.

PNWRES data was first utilized to determine a set of attitudinal, behavioral, perceptual, awareness, and consumption variables as potential segmentation variables. The demographic and socioeconomic profiles of the residents (e.g., income, education, ethnicity, etc.) as well as the characteristics of residential units (e.g, geographic, climatic, size, level of insulation, etc.) were identified as possible descriptor variables. 
Each variable was obtained by grouping a number of related variables, in the original data, into a single variable (i.e., using a cluster analysis procedure). In this context, clustering means grouping of similar classes of several variables into corresponding classes of a combined variable. Table I contains a listing of these (clustered) variables.

Segment congruence analysis as proposed by Green and Carmone (1977) and expanded here, includes two phases: (1) analysis of the multivariate system of segmentation variables (i.e., analysis of mutual association and identification of candidates variables for the distinguished segmentation variable), and (2) identification and analysis of the best segmentation variable among the candidate distinguished variables (i.e., predictability and identification of the best descriptor variables). 
TABLE I

A CONDENSED IIST OF VARIABLES USED IN THIS STUDY

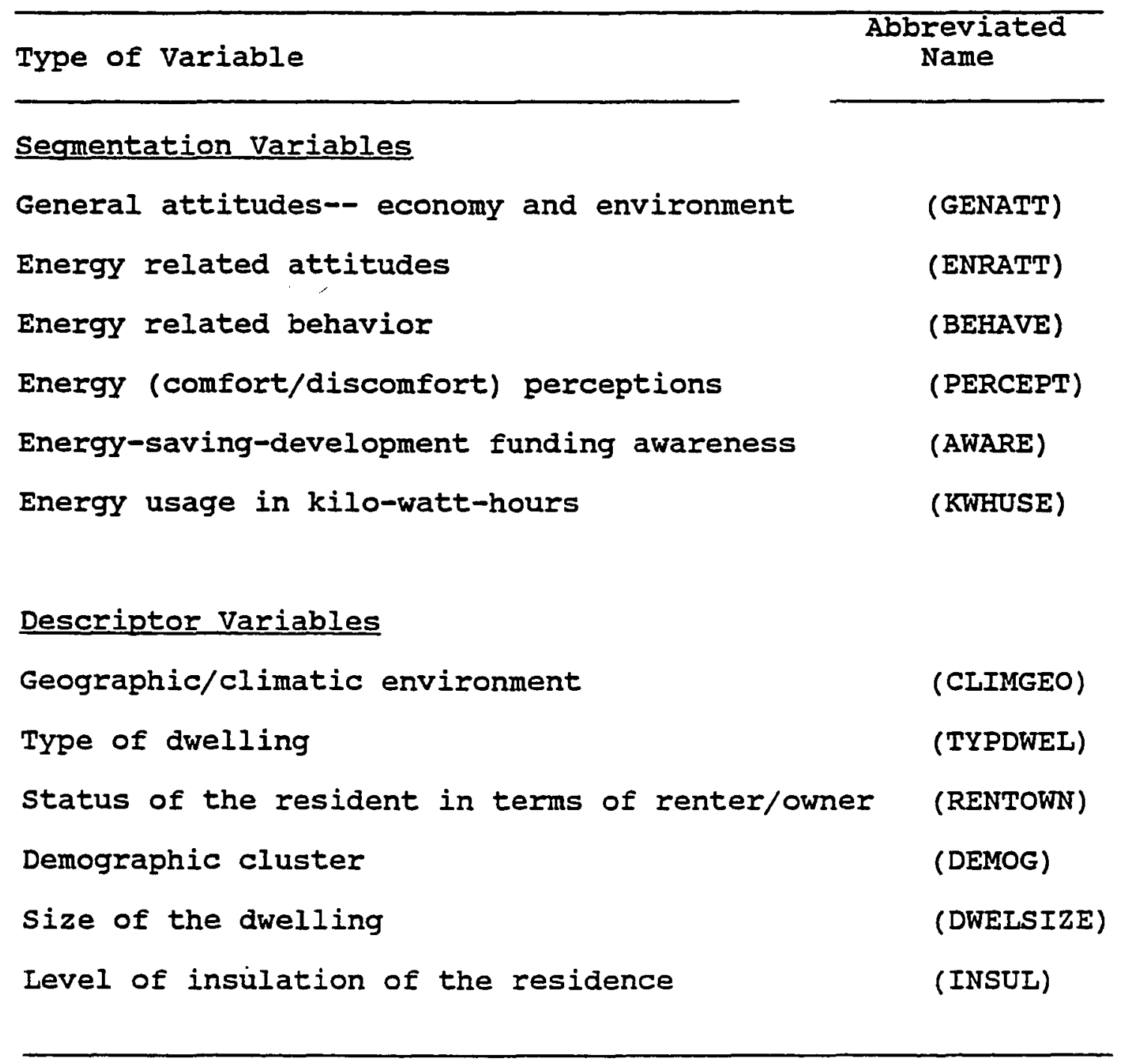


In the context of energy-usage-prediction or conservationprogram-evaluation some or all of the following questions are addressed when analyzing PNWRES data:

a) Do the attitudinal, perceptual, behavioral, awareness, and consumption variables show overlap (i.e., is it possible to gain knowledge about the class of one variable if the classes of other variables are known)?

b) Given that the overlap exists among these variables, which one variable should be considered as a candidate distinguished variable (e.g., which variable contribute the most toward the overlap etc.)?

c) Given a number of candidate distinguished segmentation variables, which one is best determined with the knowledge of the descriptor variables)?

d) Given a best segmentation variable, how well are different classes of this variable predicted by the descriptor variables?

e) Can the number of descriptor variables be reduced without significant loss in the predictive power of the prediction model?

f) What is the structure of the multivariate system composed of the descriptor variables and segmentation variable (i.e., can a simpler model of the system be developed?)

Decision makers at $B P A$, as well as local utilities in the region and even the energy industry as a whole, can benefit from such analyses in several critical ways. First, by analyzing the overlap, they will know whether the knowledge of certain variables, which can be measured with higher validity and reliability, can contribute to the knowledge of other, more vague, variables. For instance, 
consider a situation in which the consumers attitudes toward energy use are sought for a conservation program. If it is established that knowledge of energy consumption contributes significantly to the knowledge of the consumers' energy use attitudes, it may be advisable to measure the more objective energy consumption variable and assess the attitudinal variable based on the energy consumption variable.

second, the issue of predictability of segmentation variables is essential in market segmentation studies. For instance, assuming that the result of the preliminary segment congruence analysis reveals that general attitudes about the environment, energy related behavior, and energy consumption are the candidates for distinguished segmentation variables. Predictability of each of these candidate variables is then assessed and the variable that is best predicted is designated as the best (analytically derived) best segmentation variable. Third, once a set of descriptor variables are identified, particularly if a large number of such variables are considered, it is necessary to eliminate variables which do not contribute significantly to the prediction of the segmentation variable. Unnecessary variables are not only costly in terms time and computational aspects, but cloud the analysis, and in some cases lead to incorrect analyses and conclusions. 
In general, multivariate problems, including simultaneous assessment of variables in market segmentation studies, are very complex. Segment congruence analysis provides a framework to deal with this complexity which is intuitively plausible. Green and Carmone (1977) used the log-linear and logit approach to segment congruence analysis. The present undertaking employs information theory to further develop this framework.

The proposed information theoretic methodology for segment congruence analysis enables the researcher in the field of energy marketing to gain increased understanding about different segments within the residential consumers and their accessibility. Segment congruence analysis is obviously not limited to energy related issues. Any industry in which a dominant segmentation variable is not readily available can use segment congruence analysis. Even when a distinguished segmentation variable exists, these analyses will enhance understanding of the market segments and their related descriptor variables.

In the present study, several procedures available in the $\operatorname{spsS}^{\mathrm{X}}$ (1986) package are used to 1) combine classes of conceptually related variables into aggregate classes and produce variables with clustered categories: 2) perform general log-linear, hierarchical log-linear, and logit analysis in addressing the above issues; and 3) produce input files for the information theory program from the raw 
data file. A FORTRAN 77 computer program is developed to perform the information theoretic analysis.

\section{MOTIVATION FOR THE STUDY}

As an area of inquiry, market segmentation is one of the most widely investigated areas in marketing profession. It is the foundation of the modern marketing concept in which consumer needs are among the primary considerations in developing marketing strategy. Market segmentation has enjoyed considerable attention in recent years due to, at least, the following reasons:

1. As a way of thinking about underlying strategy of a corporation's marketing program, the concept can be pervasive -- it can affect every component of the program.

2. The concept of segmentation is unusual in that it is perceived both as an important building block in the marketing program of many firms and as a stimulus to the intellectual curiosity of research professionals in both the business and academic communities (Frank et al 1972, p. 246).

Segmentation studies using multiple variables are becoming more common as the marketing practitioners realize the need for further refining of market segments. When multiple variables are used, an individual may belong to two or more of the segments identified. For instance, the individual may seek fresh breath as well as decay prevention in choosing a toothpaste. Looking at this issue from the view point of product positioning, a specific brand of 
chewing gum may compete with other brands of gum as a candy substitute as well as competing with certain brands of mouthwash or with different brands of toothpaste or even cigarettes! (Arabie et al 1981)

In both segmentation and positioning contexts, marketing theoreticians and practitioners must deal with overlapping market segments. Failure to recognize that an individual belonging to the "socially aware" segment may also belong to the "health oriented" segment in the toothpaste market segmentation can result in the wrong choice in developing the marketing mix strategy. This problem becomes particularly critical when the overlap between the two segments is large. The same can be said with respect to product positioning and mixed (segmentation and positioning) situations.

Segment congruence analysis, therefore, is extremely important in situations where multiple segmentation variables are used. These situations are among the most difficult problems to deal with due to the level of complexity involved.

The proposed information theoretic method is valuable because there exists no other single method (or family of techniques) that can effectively deal with all of the aspects of segment congruence analysis. The following chapter reviews the current literature in segment congruence analysis and its relevant topics. It will be shown that, 
not only there is no unified family of techniques that can be used to address these issues, but some of the techniques are based on assumptions which may not be suitable for categorical data. Categorical data are the most widely used types of data available for defining different market segments.

Watanabe (1969) refers to information theory as a powerful tool for analyzing the organizational structure. This is because information theory offers a methodology for quantifying organization or patterning (Miller, 1953). "This creates great fascination for information theory as applied to psychology [and related social sciences]." (Attneave, 1959) This dissertation provides some additional support for these assertions. 
CHAPTER II

REVIEW OF THE LITERATURE

The process of market segmentation begins with the identification of a variable (e.g., deal proneness) or a set of variables (e.g., deal proneness and brand loyalty) as the segmentation set. These variables are drawn from a segmentation base (or basis) ${ }^{1}$. The identification of segmentation set of variables (or base) has traditionally been done either on a priori or a posteriori basis, or a hybrid of the two. (These approaches will be discussed shortly.)

Then, certain classes of a single variable (e.g., the deal prone or the non-deal prone) or combinations of classes 1 The definition of $a$ base, and its distinction from a group of variables (or even one variable), is context dependent. In general, a segmentation base is comprised of such factors as demographic/ socioeconomic characteristics, consumption patterns, personality traits, and attitudes, perceptions, and preferences. Frank, et al (1972 pp.26-28) treat variables, such as stage of life cycle and social class, as bases. Furthermore, in discussing the criteria for evaluating alternative bases for segmentation, they treat variables and bases interchangeably. Both Green and Carmone (1977) and Van Auken and Lonial (1984) treat specific variables as bases, in segment congruence analysis. 
of the set of variables (e.g., deal prone and brand loyal, deal prone and brand disloyal, etc.) are identified as the target groups (i.e., target segments). Next, a profile of each target segment is created on the basis of demographic, socioeconomic, geographic, etc., characteristics of its members. Subsequently, an attempt is made to reach (access) these segments through different marketing strategies (a market segment is called accessible if one can identify and/or target the members of that segment effectively).

\section{AN OVERVIEW OF MARKET SEGMENTATION}

Market segmentation is one of the most intensively investigated topics among both marketing researchers and practitioners today. A bibliography of publications in market segmentation during the past three decades would probably sum to several hundred pages. However, there has been relatively little substantial research to put in perspective, among certain other important issues, the role and importance of multivariate segmentation analyses.

one typology for market segmentation involves different approaches by which the segmentation variables are identified. The most common approaches include a priori, a posteriori (also referred to as post-hoc or clustering based), or hybrid (mixed) segmentation schemes (Wind 1978). In a priori segmentation, the researcher chooses some 
oluster-defining descriptor in advance, such as usage rate of a product. Respondents are then classified into clusters (or segments), such as heavy-users and light-users, and are further examined regarding their differences in other characteristics, such as demographics or benefits sought.

a posteriori (i.e, post-hoc or clustering-based) segmentation, on the other hand, starts by clustering respondents according to the similarity of their multivariate profiles regarding such characteristics as purchase behavior, attitudes, personality, life styles, and/or any other meaningful set of variables. Following this, the segments are examined for differences in other characteristics, not used in the original profile definition.

Some studies require a hybrid of the two approaches. For instance, respondents could first be grouped based on their usage rate, a priori, and then a clustering procedure could be employed to see if other segments revealing common characteristics, such as different benefit-seeking groups, emerge.

Another typology for market segmentation concerns the methods of analysis for segmentation and/or descriptor variables. These include multivariable and multivariate analysis. (Frank, et al 1972) Multivariable analysis refers to situations where a single segmentation variable is considered as the criterion (dependent) variable and several 
other variables are considered as descriptor (independent) variables. In this case, if multiple segmentation variables are identified, they are treated separately and independently of each other. (i.e., separate functions are developed for assessing the relationship between each segmentation variable and (a set of) descriptor variables.) Several quantitative techniques are available for this general conceptualization ${ }^{2}$.

The second approach, known as multivariate, requires that several segmentation variables be considered simultaneously. This approach, in general, is more complex than the multivariable approach. Here, first, a number of segmentation variables are analyzed (simultaneously) and a distinguished variable is selected. Next, relevant descriptor variables, which best determine or predict the segmentation variable, are identified.

One group of techniques is primarily suitable for simultaneous analysis of segmentation problems with intervally scaled dependent variables ${ }^{3}$. A major shortcoming

2 For instance, regression analysis, multiple discriminant analysis, multiple classification analysis (MCA), analysis of variance (ANOVA), automatic interaction detection (AID), conjoint analysis, etc.

3 These problems have been addressed in the literature using, among others, canonical correlation analysis (Alpert 1971), multivariate analysis of variance (MANOVA), additive clustering (ADDCLUS) (Arabie et. al 1981.), mathematical programing clustering (MAPCLUS) (Arabie 1977), and nonparametric multidimensional scaling. (Green and Rao 1972). 
of some of these and traditional techniques, such as regression analysis, automatic interaction detection (AID), ANOVA, and their extensions, is that most of the practical applications would violate one or more of their assumptions. Green, et al (1977) and Desarbo and Hildebrand (1980), attribute this problem to:

1. The assumption of normality for the criterion variable is unreasonable.

2. The criterion variable does not display constant variance (homoscedasticity) across variations in the predictors.

3. The model's predictions could fall outside of the range of 0 to 1 .

"Multidimensional scaling methods, [on the other hand, ] offer no analytical solution, but rather act as a transformation function to provide spatial structures underlying a data base through a series of interactive procedures ..." (Best 1975, p.16-17) Therefore, these techniques cannot be employed, for instance, to predict the behavior (or response to marketing stimuli) of a particular segment.

Another group of techniques are intended for all discrete (nominal or ordinal) data for both dependent and independent variables. It is this group of techniques that is the subject of segment congruence analysis and is explored throughout this study. In the next section, segment congruence analysis is introduced and relevant prior 
research in this area is explored. The second section describes the role of multivariate contingency tables in segment congruence analysis. Section three provides a more detailed discussion of the log-linear and logit approach to simultaneous contingency table analysis. Then, in section four, the information theoretic approach is introduced along with some of the more recent applications in this area. section five provides a brief introduction to the general probability and chi-square analysis. Finally, a summary of this chapter is presented in section six. Figure I presents a schematic view of this study.

\section{SEGMENT CONGRUENCE ANALYSIS}

Segment congruence analysis, as proposed by Green and Carmone (1977), was based on techniques for analyzing categorical data. The basic procedures for segment congruence analysis are described by Green (1977) as follows (Here, a "cluster" refers to a class of a variable $\left(x_{i}, i=1\right.$ or 2 or $3 \ldots$, or a composite class of several variables expressed simultaneously $\left(x_{i} y_{j} z_{k} \ldots, i=1\right.$ or 2 or $3 \ldots, j=1$ or 2 or 3 ..., etc.); while, a "clustering" refers to a variable $\left(x_{i}, i=1,2, \ldots\right)$, or a set of variables $\left(x_{i} y_{j} z_{k} \ldots\right.$, $i=1,2,3 \ldots, j=1,2,3 \ldots$, , etc. $)$ : 


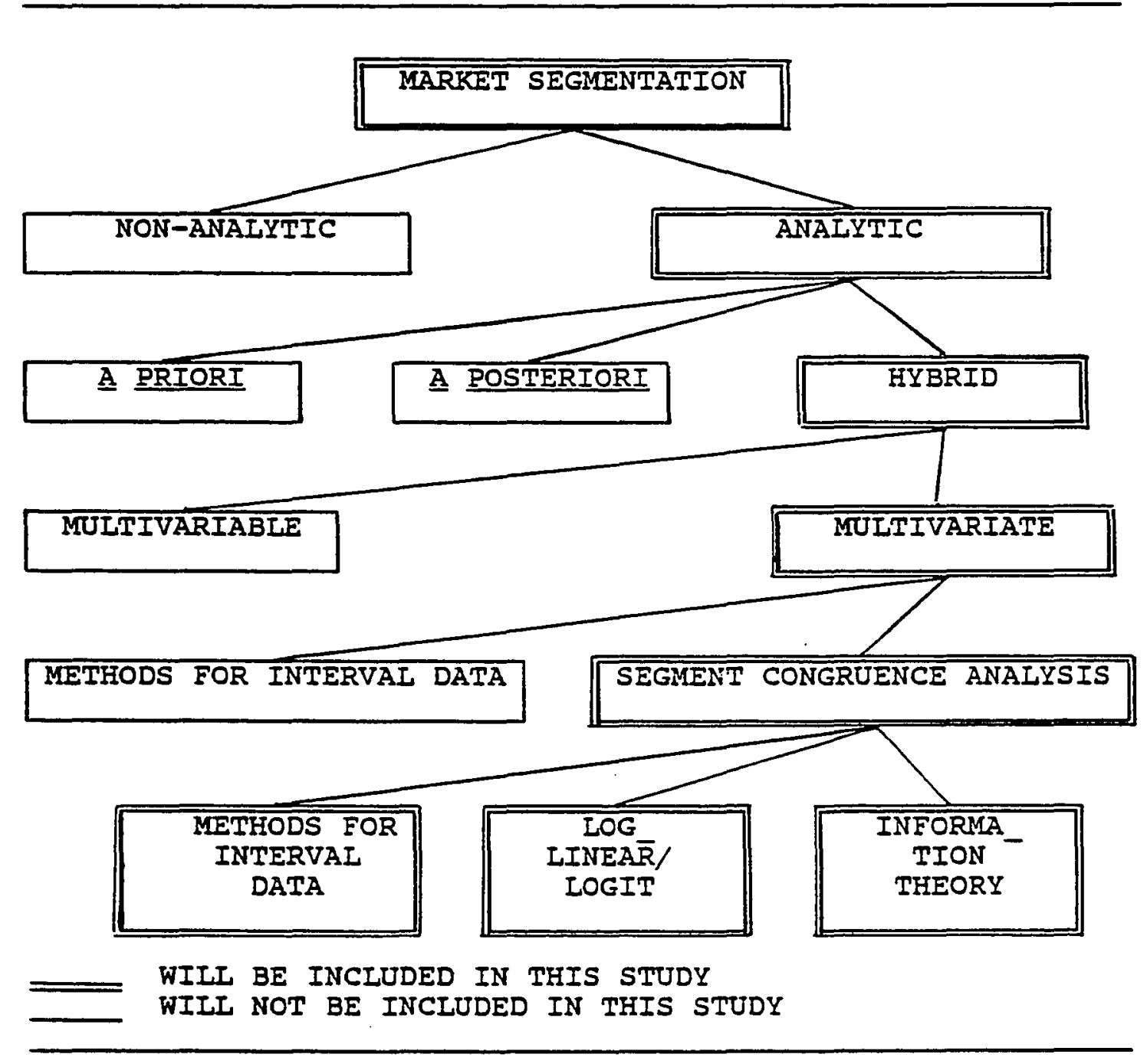

Figure 1. A schematic view of the total study. 
1. Each candidate segmentation base (battery of variables) is independently used to cluster the respondents into some specified number of groups. The number of clusters so formed may or may not be the same across bases, depending upon the researcher's objectives.

2. Each clustering serves as a categorical variable (where the clusters represent the categories), and a multidimensional contingency table is formed.

3. A variety of tests can then be carried out on the multiway table to see which clusters are related to which other ones.

4. One clustering can be chosen as the distinguished clustering and a model can be developed for predicting this clustering from clusters obtained from other bases. In this way one can ascertain how closely associated some subset of clusters may be with the distinguished clustering. If desired, clusters on non-distinguished sets can be repeated with subsets of the variables that individually evince high association with the distinguished-set clustering.

This process is presented in a diagram in Figure 2 . Green and Carmone (1977) used the log-linear models and the likelihood ratio chi-square tests of independence to conduct segment congruence analysis. This work was a continuation of an earlier paper by Green, et al (1977) in which they advocated the use of log-linear models in market structure analysis ${ }^{4}$.

In Green and Carmone's approach, the log-linear model is first utilized to determine the presence (or absence) and

4 Their work draws upon work by Bishop (1969) and Bishop, et al (1975), Goodman (1971), and Haberman (1972, 1979), who developed and used log-linear models in contingency table analysis. 
the degree of mutual association among the segmentation

variables in a multidimensional contingency table. Next, in the case that a distinguished base is selected a priori, the logit model is utilized to address predictability of this variable by the remaining segmentation variables.

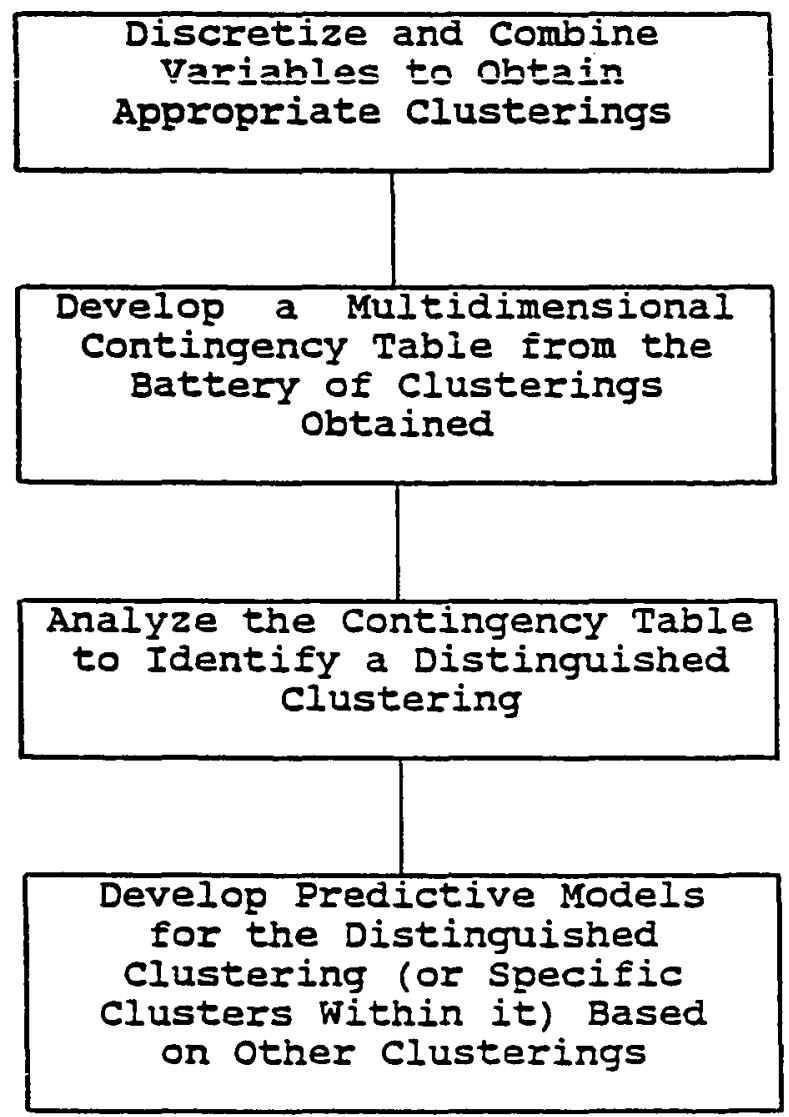

Ficure 2. Segment congruence analysis process as proposed by Green and Carmone (1977). 
Segment congruence analysis is, in fact, discrete multivariate analysis in the context of selection and analysis of market segmentation variables. As such, this concept need not be limited to the particular analyses introduced by Green and carmone (i.e., the overlap, the contributions of individual variable to the overlap, and predictability of the distinguished segmentation variable by other segmentation variables.) Some instances of additional questions follows.

1. Identification of the distinguished segmentation variable can be based on several different techniques, not all of which would necessarily identify the same variable as being central.

2. It is more theoretically sound to include exogenous descriptor variables to address predictability of the distinguished base and related questions. In traditional market segmentation, the base (or segmentation variable) is identified first and, then, a group of independent variables are sought to produce profiles of the individuals within segments.

3. Assessing the order of contributions of a large number of descriptor variables would enable the researcher to select only variables which are significant in their explanatory power.

4. Finally, structure analysis has been identified as a useful area of inquiry for marketing research (particularly in descriptive analysis of market segments (Clogg and Munch 1984). A method of structural modeling of the segmentation and descriptor variables would, therefore, be well justified.

These are some additional research questions which will be addressed in this study, along with the research 
questions raised by Green and Carmone (1977). Van Auken and Lonial (1984, pp.15-16) state that:

...selection of "the" superior segmentation base may have to be based on strategy and pragmatics...., rather than on a simplistic quantitative interpretation based on log-iinear modeling. still, the application of such modeling may be a prerequisite for [further] studies... Given associations, other issues may prevail, such as the prospect of combining segmentation bases, as well as the usage of a logit analysis on a distinguished segmentation base.

Admittedly, in many cases, the choice of segmentation variables is, indeed, dependent on the "expert's" understanding of the industry and its environment. However, it is through explorative studies such as segment congruence analysis that experts gain this understanding. In the explorative process of segment congruence analysis, it is reasonable to attempt to answer as many relevant questions as possible, because the incremental cost is small.

\section{The Role of Contingency Table Analysis}

Contingency table analysis is the building block of segment congruence analysis in the context of the two main methods discussed in this study ${ }^{5}$. Green (1977) mentioned

5 The history of contingency table analysis includes work by Pearson (1904), Fisher (1925), Yule and Kendall (1937), wilks (1943), Cramer (1946), Lazarsfeld (1942), Rao (1952), Mitra (1955), Roy and Kastenbaum (1955), Kullback (1959), Friedlander (1961), Darroch (1962), Goodman (1963 thru 1978), Bishop (1969), and many others from various fields. This effort has resulted in a rich literature in contingency table analysis. 
the use of multidimensional contingency table analysis as a general approach to multivariate segmentation studies. Even though cross-tabulation of data in the form of a contingency table is a common activity in marketing research, the applications of multivariate analysis to contingency tables have been relatively scarce (Green, et al 1977). Most practical applications using contingency tables are limited to simple cross tabulations and measures of association 6 .

Contingency table analysis for market segmentation can be performed sequentially (i.e., to address multivariable problems) or simultaneously (i.e., to address multivariate problems). The purpose of analysis can be descriptive, where interrelationships are investigated symmetrically (i.e., without a notion of causality) or predictive, where the analysis are asymmetric (i.e., causal modeling). Following are brief discussions of sequential and simultaneous contingency table analyses.

Sequential contingency Table Analysis. These techniques generally use some method of partitioning the contingency table sequentially such that some measure of dispersion within each partition is minimized. Generally,

6 A sample of some of the more sophisticated applications in market segmentation and related analyses includes the work by Myers and Nicosia (1967), Green, et al (1976 and 1977), Green and Carmone (1977), Rao and Solgaard (1977), Rao and Winter (1978), Russell and Rayan (1979), Fritzsche (1981), and Clogg and Munch (1984). 
such analyses begin by developing a contingency table which includes, as its dimensions, a criterion variable and a number of descriptor variables. Then, the contingency table is partitioned on the descriptor variable which is deemed to give the best partitioned subtables.

Each of these subtables are further partitioned based on the best descriptor variable(s) at that level, etc. For instance, the automatic interaction detection (AID) techniques partitions the data by selecting descriptor variables which minimize the variances within subtables (Green 1978) ${ }^{7}$.

Some of the related approaches to AID include THAID (Morgan and Messenger 1973), Multiple Classification Analysis (MCA) (Andrews, et al 1969), Multivariate Nominal Analysis (MNA) (Andrews and Messnger 1973), and Chi-square Automatic Interaction Detection (CHAID) (Perreault, Jr. and Barksdale, Jr. 1980). These techniques have been used in marketing research frequently ${ }^{8}$.

This techniques for contingency table analysis are invaluable. However, like most other data analysis techniques, the very property that makes them so useful in

7 An information theoretic method can be developed which is completely analogous to AID, the only difference being the fact that here the entropy of each subtable is minimized.

8 Some examples include Armstrong and Anders (1970), Assael (1970), Martin and Wright (1974), Peters (1970), Newman and Staelin (1971), Bernhardt and Kinnear (1976), and Green (1978). 
certain types of analysis limits their usefulness in certain other applications.

It should be kept in mind that the sequential partitioning methods are multivariable procedures, but not multivariate ones. All of the variables are not considered simultaneously, but rather, they are considered sequentially. Thus, a single optimal solution is not guaranteed. The partitioning which occurs in subsequent stages may be influenced by splits that have already been made. (Perreault and Barksdale 1980).

Although these techniques can be a very helpful means of reducing complexity of relationships in a large data base, and do so in a way that facilitates communication of the results, one should remember that the higher order relations will be overlooked (Perreault and Barksdale 1980). This may severely distort the analysis and yield misleading results.

Simultaneous Contingency Table Analysis. This type of analysis is not only concerned with the direct (first-order) interactions between variables but also with relationships which are a result of higher-order interactions between multiple variables. Three variables may have significant two way relationships but insignificant three way (or second-order) correlations (LaGrace 1974). In this case the researcher is able to exclude the three way interactions and 
produce a simpler model for description or prediction of the essential variables.

The analysis of interactions in contingency tables

have been performed through several methods. These techniques include weighted least squares, dummy variable analysis, graph theory, general probability and chi-square analysis, log-linear models, and information theory.

The most relevant techniques among the above will be discussed throughout the remainder of this review in the following manner. First, the general $10 \mathrm{~g}$-linear/logit approach will be discussed. Next, the information theoretic approach will be covered. Then, other techniques which are particularly suitable for contingency table analysis will be explored.

THE LOG-IINEAR/IOGIT APPROACH

Green and Carmone (1977) proposed the general loglinear and the logit models for segment congruence analysis. This work was a continuation of an earlier paper by Green, 9 For excellent discussions of these techniques, see the following references: weighted least squares (Grizzle, et al 1969, and Theil 1970), dummy variable analysis (Miller and Erickson 1974, and Andrews, et al 1969), graph theory (Davis 1975) general probability and chi-square analysis (Bartlett 1955, Simpson 1951, and Lewis 1962), log-linear models (Goodman 1963, 1964a, 1964b, 1964c, 1964d, 1965a, 1965b, 1965c, 1969a, 1969b, 1972, Bishop, et al 1969, and 1975, and Haberman 1972 and 1979), and information theory (KIir 1976, Cavallo and Klir 1979, 1981, Conant 1974, Broekstra 1978, 1981, 1982, Krippendorff 1979, 1981, and Abrahamse and van Bueren 1980). 
et al (1977) in which they advocated the use of log-linear models in market structure analysis. Their work draws upon work by Bishop (1969) and Bishop, et al (1975), Goodman (1971a), and Haberman (1972, 1979), who introduced loglinear models as a tool for contingency table analysis. A brief description of log-linear and logit models appears in Appendix I.

Relevant Applications of the General Log-Linear Model

In Green and Carmone's approach, the log-linear model is first utilized to determine the presence (or absence) and degree of mutual association among the segmentation variables in a multi-dimensional contingency table. Data collected in a 1975 survey of 534 respondents' views about certain aspects of their automobile insurance was used by Green and Carmone. This data was subsequently analyzed through factor analysis (in order to combine certain related variables into single variables) and (on an a priori basis) four segmentation variables were identified. These segmentation variables included "insurance supplier," "psychographic," "image," and "demographic" variables. Then the following hypotheses were tested through the general log-linear model:

a) All four clusterings are mutually independent.

b) Insurance supplier is independent of the other three segmentations. 
c) Psychographics are independent of the other three segmentations.

d) Image is independent of the other three segmentations.

e) Demographics are independent of the other three segmentations.

The general log-linear independence model (see Appendix I) was then utilized to demonstrate the following results:

1. The four segmentation variables exhibit significant mutual association (at the alpha risk level $<=.05$ ).

2. None of the four segmentations is independent of the other three.

Then, they used the drop in the chi-square between the mutual independence model and that of conditional independence models to conclude that the "image" base demonstrates the highest association with the remaining three bases. Therefore, they suggested that if one were selecting bases on a posteriori basis, the image base would be the most attractive candidate.

Next, they utilized the logit model to address the predictability of this variable by the remaining segmentation variables. This will be further discussed in the next section.

Van Auken and Lonial (1984) used Green and Carmone's approach to analyze different segmentation bases for dog 
food. Their study involved a convenience sample of ninetyfour dog food purchasers (This would, of course, raise questions regarding representativeness of the sample and reliability of the results, but, as an application of segment congruence analysis methodology, it is noteworthy.) Sixteen attitudinal variables, eleven benefit variables, and five problem concerns were measured, and usage data was also collected on each respondent

Each group of variables for attitudes, benefits, and problems were combined into dichotomous clusterings (This, further, raises issues concerning spurious or suppressive results created through artificial collapsings of classes of variables). For this purpose, they used factor analysis and a minimum-variance hierarchical clustering algorithm.

Using the same four hypotheses as Green and Carmone's, they concluded that the four segmentation bases attitudes, benefits, problems, and usage, are mutually associated (In reality, in spite of their claim that all four variables were mutually associated, the hypothesis of mutual independence is rejected even if only two of the variables are significantly associated.) Then using the one-variable independence log-linear models, they concluded that the problems base was independent of the other three, but attitudes, benefits, and usage were not independent of the others. 
Then, by calculating the differences between the chisquare of the mutual independence model and the one-variable independence models, they concluded that the benefits base was the distinguished base. But they also used the USAGE variable for further analysis, regarding predictability, using the main-effects logit model. They further interpreted the logit model to prioritize the effect of the remaining variables. Benefits, attitudes, and problems contributed to explaining usage, in the order given here. Other applications of the general log-linear model include Goodman (1970). The first application describes the general approach to estimation and hypothesis testing for two, three, four, and five variables (Goodman 1970). It also reanalyzes data pertaining to five dichotomized variables that classify individuals according to their information sources (e.g., newspapers, radio, books, etc.) and knowledge of cancer. He demonstrates that the logIinear models fit the data better than those obtained in earlier published analysis of the same data.

Another application by Goodman (1971b) illustrates stepwise procedures and estimation methods for building models of multiple classifications. These procedures are somewhat analogous to the classical stepwise regression methods for adding terms (forward selection) and deleting terms (backwards elimination) for qualitative models. This application gave rise to the now well-known hierarchical 
log-linear models. These methods are illustrated by Goodman with a classification of 1008 consumers according to their preference for laundry detergents, previous use of a certain brand, water temperature, and water softness.

In another application, Goodman (1972) shows how recursive and nonrecursive path diagrams and more general systems can be formulated and tested using the general loglinear model. This approach is illustrated in a reanalysis of data from wilner, et al (1955) study of the contact hypothesis, which states that the close proximity of whites and blacks in integrated housing increases favorable interracial sentiments through indirect effects by other variables such as contact and local norms.

\section{Relevant Applications of the Logit Model}

Predictive models for nominally scaled dependent variables have traditionally been based on the techniques with assumptions which are more appropriate for interval data. The traditional assumptions underlying regression analysis are violated when the dependent variable is categorical (Goodman 1973). If only the independent variables are nominally scaled, techniques for quantitative data, such as regression analysis, can be utilized by creating dumm variables which partition the nominal variable(s) into a set of sequential binary variables. Aside from the fact that in most cases it becomes extremely 
cumbersome to interpret the regression results, the multicolinearity among these variables cannot conveniently be attributed to specific sets of variables.

Goodman (1972), building upon earlier work by Bishop (1969) and others, proposed a modified multiple regression approach for analyzing data of this kind. This approach uses the "logit model," a special case of the general loglinear models, and the maximum likelihood estimation procedure instead of least squares. For a description of the logit model, refer to Appendix I.

To illustrate the logit model, Goodman (1972) reanalyzed the famous study of the American Soldier by Stouffer, et al (1949), Coleman (1964), Zeisel (1968), and Theil (1970). Stouffer, et al (1949) and Coleman (1964) used regression analysis and analysis of variance to analyze this data. Coleman admits that the model differs from the actual in certain systematic ways. The variances of the estimates of the main effects would have been smaller had they used a more efficient method estimation method such as the maximum likelihood estimation methods (Goodman 1972). Zeisel (1968) and Theil (1970) used the weighted logisticcurve-transformation regression model to analyze the same data. Again, the estimates by these models have somewhat larger variances than the maximum likelihood estimates by Goodman (Rao 1965). 
Goodman (1978) presented comparisons between Goodman's logit model and the dumny-variable regression model. He concluded that, though both models yield meaningful estimations, the logit model fits the data (the American Soldier data) substantially better than the corresponding aditive probability models.

Chi-square measure is used to evaluate logit models. obviously, small values of chi-square would be sought in order to establish that the logit model accounts for the variations in the data. Haberman (1982) proposed two additional measures (i.e., a measure of dispersion and one of concentration) as criteria for evaluating logit models. He gave the entropy measure as a measure of dispersion between the actual and estimated frequencies and the squared Euclidean distance between them as a measure of concentration. These measures are presented below.

$$
\begin{aligned}
& \text { (1) } H(f, F)=\sum_{i} \quad f_{i} \log \left(f_{i} / F_{i}\right) \\
& \text { (2) } C(f, F)=\sum_{i} \quad\left(f_{i}-F_{i}\right)^{2},
\end{aligned}
$$

where

$$
\begin{aligned}
f_{i} & =\text { Actual frequency in } i \quad(i=1,2, \ldots, N) \\
F_{i} & =\text { Estimated frequency in } i(i=1,2, \ldots, N) \\
H(f, F) & =\text { Measure of Dispersion } \\
c(f, F) & =\text { Measure of Concentration }
\end{aligned}
$$


Applications of the logit models in marketing have been extremely rare. Referring back to the work by Green and his colleagues; Green, et al (1977) advocated the use of these techniques in marketing. Applying the logit model to the insurance company data, Green and Carmone (1977) assessed the predictability of an a priori selected distinguished base (i.e., the insurance supplier variable). They noted that "the objective of logit analysis is to construct a model for predicting the probability of membership in the segment representing the sponsoring firm's customers, given knowledge of the respondent's membership in the segments developed from the other three bases: psychographics, image and demographics." (Green and Carmone 1977, p.220). Other applications of the general $\log -1$ inear and logit models in marketing include consumer resource allocation, (Batsell 1980), individual and aggregate consumer brand choice (Batsell and Lodish 1981, Chapman and Staelin 1982, Currim 1981 and 1982, Gensch and Recker 1979, Jones and Zufryden 1980, Punj and Staelin 1978), marketing data analysis in general (Flath and Leonard 1979, Silk and Urban 1978), consumer response models (Hauser and Urban 1977), information load and information processing (Malhotra $1982 \mathrm{a}$ and 1982b, Malhotra, et al 1982). 
THE INFORMATION THEORETIC APPROACH

A concept which is fundamental in general system theory and methodology and in system theoretic data analysis in particular is the concept of a system. "In general, a system is an abstraction distinguished on an object by an observer, which reflects the interaction between the observer and the object" (KIir 1986, p. 267). In the data analytic sense, this abstraction is conceived as a set of variables together with a characterization of relationship, dependency, correlation, or any other expression of a constraint among the variables.

Constraint analysis as proposed by Ashby (1964), and later developed by $\mathrm{KI} i r$ and his colleagues, evaluates a system in terms of the information contained in its given subsystems. "Information is considered here in terms of the reduced uncertainty it produces in the overall system infarred (Klir 1986, p. 269)."

To make this principle [of the information content within a system] operational, a unique and well justified measure of uncertainty must be determined for the mathematical formalism employed (Klir 1986, p. 269).

Such measure exists in statistical information theory. Herniter (1973) gives the following statement about information theory's measure of uncertainty: 
The great advance provided by information theory lies in the discovery that there is a unique, unambiguous criterion for the "amount of uncertainty" represented by a discrete probability distribution, which agrees with our intuitive notions that a broad distribution represents more uncertainty than does a sharply peaked one, and satisfies all other conditions which make it reasonable.

\begin{abstract}
statistical information theory draws its foundations from "entropy" in thermodynamics. Entropy, as used by Shannon (Shannon and Weaver 1949), refers to uncertainty associated with a signal transmitted through a channel of communication. The amount of "information" in a message is defined as the reduction of the uncertainty achieved by receiver of the message. "It has been proven in several alternative ways that shannon entropy is the only function that possesses all known properties for a probabilistic measure of uncertainty." (KIir 1986, p. 269)

An extensive body of literature has been devoted to the study of the relationship between entropy and information (Stumpers 1953; Bartlett 1955; Brillouin 1956; and Cherry 1952; have produced extensive bibliographies concerning this relationship.)
\end{abstract}

The work by McGill (1954), Kullback (1959), Garner (1962), Theil (1967), Ku and Kullback (1968), and many others, has further developed information theory and its application to social sciences. 
Applications of the information theoretic models to marketing include the following: Bernhardt and Mackenzie (1968), Herniter (1973 and 1974), Bass (1976). A brief summary of the concept and some extensions of information theory appears in Appendix II.

This study employs Shannon's (Shannon and Weaver 1949) entropy measure as a measure of uncertainty (and hence the information that can be acquired when this uncertainty is reduced in a system) to assess discrete multivariate systems. It will be demonstrated that the data analysis properties of information theory, also referred to as uncertainty analysis (Conant 1974) and constraint analysis (Broekstra 1978, 1981), provide a powerful vehicle for assessing interrelationships and the structure of a system.

Some Important Properties of Information Theory

Recent developments in information theoretic methods for constraint analysis, reconstructability analysis, structural modeling (Conant 1974, Klir 1976, Cavallo and Klir 1979, 1981, Broekstra 1981, and Krippendorff 1981) have promised an approach to contingency table analysis different from and potentially superior to the general log-linear and logit methods. These methods enable the researcher to assess the structure of the data with respect to their mutual association, the strength of each variable's contribution to the total mutual association, and 
predictability of each variable based on the other variables.

This is done by utilizing the decomposability property of the information theoretic measures which enables assessment of total interactions as well as partial interactions among variables. The present undertaking will demonstrate that segment congruence analysis can be performed using the information theoretic approach.

The most important properties of the entropy measure (Horowitz and Horowitz 1976) include the following:

1) As the probabilities of possible states tend to equalization, the entropy of the system approaches its maximum.

2) Between two systems at maximum entropy the one with a larger number of states has a higher entropy measure.

3) Entropy of a system is between zero and the logarithm of the number of possible states of the system.

$4)$ It is possible to calculate all conditional entropies including the measures parallel to the Bayesian posterior probabilities.

5) The entropy measure is fully decomposable, enabling the calculation of entropies within and between groups in a manner similar to ANOVA.

It has been shown that information theoretic data analysis and the analysis of variance are analogous. Uncertainties can be estimated directly from variances in certain cases, using the relationships between the two. (Garner and MCGill 1956, Attneave 1959). The decision as to 
which of the techniques to use depends partly on the properties of the criterion variable.

Only uncertainty analysis may be used with nonmetric criterion variables since uncertainties are dimensionless (using no metric), however, uncertainty analysis has a generality which may make it useful even when variances can be computed. (Garner and MCGill 1956, p. 219)

Considering the fact that research in social sciences (including marketing) uses qualitative data extensively, information theoretic analysis can be applied in this area. This was the primary motivation for work in information theory by a great number of researchers.

\section{Information Theoretic Definitions of a Leading Part}

The concept of leading part was initially introduced by von Bertalanffy (1968) in connection with tendency of systems for segregation and/or centralization. He defined this concept in the following manner:

\footnotetext{
Suppose that the coefficients of one element, $\mathrm{P}_{\mathrm{S}}$ ' are large in all equations [of a system of differential equations, as a model of a system under investigation, ] while the coefficients of the other elements are considerably smaller or even equal to zero. ... We may call the element $P_{s}$ a leading part, or say that the system is centered around $\mathrm{P}_{\mathbf{S}}$. (von Bertalanffy 1968, p.71)
}

The significance of a leading part is in that a small change in this part (e.g., element, variable, or group of variables) may cause a significant change in the whole 
system. In information theoretic data analysis, similar to the other descriptive data analysis methods, much attention has been given to identifying one (or a set of). most significant variable(s)

Several schemes for information theoretic definition of the leading part have been developed by Zwick (1979), which will be further extended and presented briefly in this section. (The reader unfamiliar with information theory should review Appendix II at this point). The information theoretic leading part can be defined according to, at least, four perspectives:

1. The part which makes the highest contribution to the transmission.

2. The part which makes the highest contribution to the joint variability (systematic entropy) in the system.

3. The part which makes the highest contribution to the overall uncertainty in the system.

4. the part which has the greatest number of interactions with the other variables.

The mathematical expressions for these four schemes are developed in Chapter 3 (Research Questions, Methodology, and Procedures), in the context of segment congruence analysis. Those expressions can easily be generalized to include all multivariate systems. Therefore, they will not be repeated here. 
Statistical Significance of Informational Measures

Based on Wilks' (1935) work and Miller's (1953) proof, informational measures are known to be asymptotically chisquare distributed. This work facilitated the application of information theory to social science problems, where sampling is prevalent, and the analyses are performed to assess the behavior of the population from which the sample is drawn.

Significance Test for the Transmission Measure. With $\mathrm{N}$ denoting the sample size, the exact likelihood ratio chisquare value for the information theoretic measure of transmission is 2.N.T.LOG(2). Attneave (1959), using the then much more common $\mathrm{x}^{2}$ (Pearson's chi-square) states that this relationship is an approximation. However, in Table II (Part a) derivation of likelihood ratio chi-square from transmission is presented and it is shown that this relationship is exact. Therefore, using information theoretic transmission measures provides the researcher with tests of independence, using the likelihood ratio chisquare with degrees of freedom given by Broekstra (1981, p.51) and presented below:

(3) $\operatorname{df}(T)=-\sum_{i}\left(\left|s\left(V_{i}\right)\right|-I\right)+|A|-I$ 
In equation $3, i=$ number of variables in $T, s\left(v_{i}\right)=$ number of states (classes) in variable $i$, and $|A|=$ number of aggregate states (cells) in the contingency tables.

Significance Test for the Entropy Measure. In principle, the chi-square tests are capable of entertaining any null hypothesis with respect to the distribution (or generating function) of the expected frequencies. Blalock (1972, p.275) states that: "The chi-square test is a very general test that can be used whenever we wish to evaluate whether or not frequencies which have been empirically obtained differ significantly from those which would be expected under certain set of theoretical assumptions." The chi-square test of independence assumes that the expected (cell) frequencies are products of the marginal frequencies. This (independence) is an implicit assumption in the calculation of the transmission measure.

In order to test the entropy measure, one can calculate the chi-square value using the empirically observed frequencies against a uniform distribution. The null hypothesis would then assess the departure from the uniform distribution (i.e., maximum entropy) for the observed frequencies. The calculation of the likelihoodration chi-square for measures of entropy is presented in Table II (Part b)

As all information theoretic measures can be converted to entropy terms (see, for instance, Krippendorff 1979), 
this procedure provides a test of significance for all such measures. The literature search, conducted as part of this research, has not found any previous work utilizing this relatively trivial but useful conclusion.

Degrees of freedom in this case are calculated as $n-I$, where $\mathrm{n}$ is the number of cells in the contingency table (the only constraint being the sum of relative frequencies (i.e., probabilities must) be one. For the more complex measures (e.g., systematic entropy), by analogy to the degrees of freedom for complex transmissions (Broekstra 1981), the algebraic sum of the degrees of freedom would produce the total number of degrees of freedom.

The derivation in Table II (Part b) has an implication that can prove to be quite useful. By performing the appropriate linear transformation on $\mathrm{H}$, one can obtain the likelihood-ratio chi-square for the entropy measure. Though, the likelihood-ratio chi-square measure has been used extensively for the transmission based measures, it has not been used for statistical assessment of information theoretic measures other than transmission, such as entropy. This derivation shows that $L^{2}$ for the transmission measure can be used to test departure from independence while $\mathrm{L}^{2}$ for the entropy measure can be used to test departure from maximum entropy. 
TABLE II

RELATIONSHIP BETWEEN LIKELIHOOD-RATIO CHI-SOUARE

AND THE INFORMATION THEORETIC MEASURES

(Part a) Calculation of the likelihood-Ratio chi-square for the transmission measure

$$
\begin{aligned}
& I^{2}=2 \sum O \log (O / E) \text {, } \\
& \text { where, } 0=f_{i j}=n_{i j} P_{i j} \text {, and } E=F_{i j}=n_{i j} P_{i \cdot} P_{\cdot j} \\
& =2 \Sigma_{i} \Sigma_{j} n_{i j} P_{i j} \log \left(n_{i j} P_{i j} / n_{i j} P_{i \cdot} P_{\cdot j}\right) \\
& =2 N \Sigma_{i} \Sigma_{j} P_{i j} \log \left(P_{i j} / P_{i \cdot P} \cdot j\right) \\
& =2 N \Sigma_{i} \Sigma_{j} P_{i j}\left(\log P_{i j}-\log P_{i \cdot}-\log P_{\cdot j}\right) \\
& =2 N\left(\Sigma_{i} \Sigma_{j} P_{i j} \log P_{i j}-\quad \Sigma_{i} P_{i .} \log P_{i \cdot}-\Sigma_{j} P_{\cdot j} \log P_{. j}\right) \\
& \left.T=\Sigma_{i} \Sigma_{j} P_{i j} \log _{2} P_{i j}-\Sigma_{i} P_{i \cdot} \log _{2} P_{i}-\Sigma_{j} P_{\cdot j} \log _{2} P_{\cdot j}\right) \\
& \text { therefore; } \\
& L^{2}=2 N \log (2)(T)
\end{aligned}
$$

(Part b) Calculation of the likelihood-Ratio chi-square for the entropy measure

$$
L^{2}=2 N \quad \sum_{i} \Sigma_{j} \quad P_{i j} \log P_{i j} /(1 / A)
$$

where

$A=$ Total number of states (i.e., number of cells in the contingency table

$$
\begin{aligned}
& =2 N \Sigma_{i} \Sigma_{j} P_{i j} \log P_{i j}+2 N \Sigma_{i} \Sigma_{j} P_{i j} \log (I / A) \\
& =2 N \log (2) \Sigma_{i} \Sigma_{j} P_{i j} \log _{2} P_{i j}-2 N \log \left(A \Sigma_{i} \Sigma_{j} P_{i j}\right. \\
& =-2 N \log (2)(H)+2 N \log (A) \\
& =-2 N \log (2)\left[H-H_{M A X}\right]
\end{aligned}
$$


Information Theory as a Tool for segment congruence Analys is Information theory strengthens segment congruence analysis in several critical ways. (1) It readily provides a measure of the strength of association as well as addressing the question of statistical existence of the association. (2) The leading part identification schemes provide a tool of identifying several candidates for segmentation variables. (3) It avails the structure analysis techniques, which are very well developed using the information theoretic ideas, to researchers in marketing and other social sciences. Following is a brief discussion of these features and their critical role in data analysis. Information Theoretic Measures of Association as a measure of overlap in multivariate data. As will be seen in the methodology chapter, the general log-linear approach hypothesize a model for the data (e.g., all variables are independent). This model is then used to generate the expected cell frequencies, using the functions of the appropriate marginals, or in some cases (when no direct estimations are possible-- to be explained later) an iterative process. Next, the expected cell frequencies, generated based on the model, are compared with the actual cell frequencies to draw statistical inference regarding the goodness-of-fit of the model. 
But this only assesses the existence (or lack of existence) of a particular hypothesized relationship in the data. Blalock (1972, p.291) states the following:

We have set up null hypotheses to the effect that $[$ e.g.,] there is no relationship and have then tried to reject these hypotheses. But just how much have we accomplished when we are able to reject? we refer to a relationship as being statistically significant when we have established, subject to the risk of a type I error, that there is a relationship between the two [or more] variables. But does this mean that the relationship is significant in the sense of being a strong relationship or an important one? Not necessarily. The question of the strength of a relationship is a completely different question from that of whether or not the relationship exists.

Although many measures of association are available for assessing the strength of relations, the information theoretic measures of association (e.g., transmission and systematic entropy) have the following advantages:

a) They have definite minimum and maximum values.

b) There exists a direct formula which produces the likelihood-ratio chi-square for each measure.

b) Different models can be hypothesized using information theoretic models.

No other technique (e.g., the general log-linear models or the chi-square alone) has all of the above properties. The log-linear approach only provides a scheme of generating the cell frequencies, and likelihood-ratio chi-square alone is not nearly as flexible (nor as established) in model generation. 
Use of the concept of Leading Part in the Identification of Alternative candidates for the Distinguished Segmentation Variable. There exists a great deal of controversy concerning whether the post-hoc (or even the hybrid) segmentation schemes identify meaningful market segments. (e.g., Green 1977, wind 1978) This is partly due to the fact that most clustering-based segmentation methods identify a single variable (or clustering) as the segmentation base, then, proceed to assess their predictability through the background variables. The four definitions of leading part enable the researcher to, first, identify several candidate segmentation variables (or clusterings). Then, through the assessment of predictability of these candidate variable, a distinguished variable (or clustering) is identified. This is obviously a critical advantage of using the proposed information theoretic approach.

Advances in Information Theoretic Structure Analysis Useful in Segment Congruence Analysis. As a descriptive family of techniques structural modeling is invaluable in any multivariate analysis context. For instance, one of the reasons for clustering-based techniques' inability to produce meaningful market segments can be attributed to the lack of a thorough understanding of the structure and strength of interrelationships among the variables in the models analyzed. 
Researchers in general system methodology have consistently been at forefront of structural modeling techniques. Constraint analysis, dependency analysis, reconstructability analysis, etc. are familiar terms in general system methodology. The primary tool used for these analysis techniques is information theory.

The log-linear based hierarchical modeling or the general probability models are still in infancy. The methods used to generate and assess models parallel those of information theoretic approach, however, information theory is more established and defined. Information theory, therefore, offers a valuable family of techniques to the researcher in marketing, which improves his/her understanding of the complex multivariate systems. This underutilized approach can enable the marketing practitioners to make better-informed decisions.

THE GENERAL PROBABILITY AND CHI-SQUARE APPROACH

In addition to $\log -\mathrm{linear}$ and information theoretic methods, a variety of other techniques have been used to address mutually associated variables. Among these methods, only the general probability and chi-square analysis warrants inclusion in this review. Two reasons justify this inclusion: I) this approach has occasionally been used to analyze contingency tables directly (Goodman 1971a, 1974, 
Dempster, et al (1977), Clogg and Munch 1984), and 2) the chi-square measure is used in both log-linear and information theoretic approaches to assess statistical significance for different models.

The general probability approach primarily deals with calculations of joint and conditional probabilities of occurrence of mutually dependent events. Principles of this approach are present in (and used by) both the general loglinear models and the information theoretic models. Some recent work in this area is attributed to Goodman (1974), Dempster, et al (1977), and Clogg and Munch (1984), among others.

Clogg and Goodman (1985) developed the simultaneous latent structure analysis model in general terms. This model is a probability based model which searches for unobservable (latent) variable(s) underlying the data. These models could also be used for studying structural models by searching for the observable variable(s) that are best determined by the other variables, however, no applications of this was found. 
Chi-Square Test of Independence. In general terms, independence means that the probability of one event is not affected by occurrence or non-occurrence of another event. Variables in a contingency table are independent if the probability of occurrence of each composite class (e.g., $\left.P_{i j k}\right)$ is equal to the product of the relevant marginal probabilities (i.e., $P_{i . . .}{ }^{*} . j .{ }^{*} P_{. K}$, where, $P_{i . .}=\Sigma_{j} \Sigma_{k} P_{i j k}$, etc.) .

To test the hypothesis that an observed crossclassification fits the independence model, the estimated expected cell-frequencies are calculated from the marginal frequencies:

$$
\text { (4) } \quad F_{i j k}=n *\left(f_{i} . . / n\right) *\left(f_{\cdot j \cdot j} / n\right) *\left(f \ldots k^{/ n}\right)
$$

These expected values are then compared with the observed values using either the Pearson chi-square statistic (2), or the likelihood-ratio chi-square (3):

$$
\begin{aligned}
& \text { (5) } x^{2}=\Sigma_{i} \Sigma_{j} \Sigma_{k}\left(f_{i j k}-F_{i j k}\right)^{2} / F_{i j k} \\
& \text { (6) } I^{2}=2 \Sigma_{i} \Sigma_{j} \Sigma_{k} f_{i j k} \log \left(f_{i j k} / F_{i j k}\right)
\end{aligned}
$$

"Although less widely known than $G^{2}{ }^{2}$ [Pearson $x^{2}$ ], the likelihood ratio chi-square has the advantage of being divisible into additive portions, much as the total sum of squares in the analysis of variance is partitioned." 
(Reynolds 1977, p.8-9) "Both statistics are asymptotically distributed as chi-square, with $n$ - p degrees of freedom, where $n$ is the number of cells in the table, and $p$ is the number of independent parameters fitted." (Desarbo and Fildebrand 1980, p.44)

\section{SUMMARY}

This chapter produced a review of the literature in segment congruence analysis and other relevant concepts and techniques. First, it was established that in order to perform segment congruence analysis in its intended form, a multidimensional contingency tables of segmentation variables must be developed. This highlighted the significant role of multivariate discrete analysis using contingency tables. It was then demonstrated that techniques for sequential analyses or those which assume interval dependent variables are not suitable for segment congruence analysis.

Next, simultaneous discrete multivariate analysis was introduced, followed by concepts and applications of loglinear/logit, information theoretic, and general probability methods of performing such analysis. It was seen that, in spite of great interest in the information theoretic approach, actual applications of these techniques in marketing are scarce. Considering that segment congruence 
analysis seems to be a logical area of application for information theory, it is justified to use and compare this technique with other traditional methods.

Table III presents an outline of different sections of this literature review and a sample of relevant publications in each area. 
TABIE III

SELECTED PUBLICATIONS IN THE AREAS CENTRAL TO THE PRESENT STUDY

\begin{tabular}{|c|c|c|}
\hline TOPIC & SOURCE & DATE \\
\hline $\begin{array}{l}\text { Segment } \\
\text { Congruence } \\
\text { Analysis }\end{array}$ & $\begin{array}{l}\text { Green \& Carmone } \\
\text { Green } \\
\text { Arabie, et al } \\
\text { Van Auken \& Lonial } \\
\text { Harmon, Zwick, \& Hosseini }\end{array}$ & $\begin{array}{l}1977 \\
1977 \\
1981 \\
1984 \\
1986\end{array}$ \\
\hline - Contingency Table & Analysis: & \\
\hline $\begin{array}{c}\text {-Log-Linear } \\
\text { Approach }\end{array}$ & $\begin{array}{l}\text { Haberman } \\
\text { Goodman } \\
\text { Bishop } \\
\text { Bishop, et al } \\
\text { Green, et al } \\
\text { Green \& Carmone } \\
\text { Malhotra } \\
\text { DeSarbo \& Hildebrand } \\
\text { Currim } \\
\text { Batsell \& Iodish } \\
\text { Batsell \& Itaelin } \\
\text { Chapman \& Stan } \\
\text { Gensch \& Recker } \\
\text { Jones \& Zufryden } \\
\text { Punj \& Staelin } \\
\text { Flath \& Ieonard } \\
\text { Silk \& Urban } \\
\text { Hauser \& Urban }\end{array}$ & $\begin{array}{l}1972,1979 \\
1963-1972 \\
1969 \\
1975 \\
1977 \\
1977 \\
1982 \\
1980 \\
1981,1982 \\
1980 \\
1981 \\
1982 \\
1979 \\
1980 \\
1978 \\
1979 \\
1978 \\
1977\end{array}$ \\
\hline $\begin{array}{c}\text {-Information } \\
\text { Theoretic } \\
\text { Approach }\end{array}$ & $\begin{array}{l}\text { Klir } \\
\text { Cavallo \& Klir } \\
\text { Conant } \\
\text { Broekstra } \\
\text { Krippendorff } \\
\text { Abrahamse \& van Bueren } \\
\text { Bernhardt \& Mackenzie } \\
\text { Herniter } \\
\text { Bass }\end{array}$ & $\begin{array}{l}1976-1981 \\
1979,1981 \\
1974-1982 \\
1978-1981 \\
1976-1981 \\
1980 \\
1968 \\
1973,1974 \\
1976\end{array}$ \\
\hline $\begin{array}{l}\text {-General } \\
\text { Probability } \\
\text { Chi-square } \\
\text { Analysis }\end{array}$ & $\begin{array}{l}\text { Bartlett } \\
\text { Simpson } \\
\text { Goodman } \\
\text { Clogg \& Goodman } \\
\text { Dempster, et al } \\
\text { Clogg \& Munch }\end{array}$ & $\begin{array}{l}1935 \\
1951 \\
1963-1978 \\
1985 \\
1977 \\
1984\end{array}$ \\
\hline
\end{tabular}


CHAPTER III

RESEARCH QUESTIONS, METHODOLOGY, AND PROCEDURES

This chapter is composed of three parts: research questions, methodology, and description of the data used. First, a description of the research questions addressed in this study is presented. Next, the information theoretic and the log-linear/logit approaches are described in the context of segment congruence analysis is given. Finally, a description of the study and the procedures employed for selection and aggregation of variables is provided.

\section{RESEARCH QUESTIONS}

This section describes the research questions proposed for conducting segment congruence analysis and their significance in general and as applied to the data used (i.e., the residential energy survey data). When multiple segmentation variables are used, the researcher must investigate the implications of interactive relationships among these variables (i.e., mutual causal effects, strength of relations, predictability of each variable or set of 
variables, reduction of the predictor-variable set, and the structure of the segments). This investigation is conducted in this study through two distinct phases, (I) analysis of the overlap, and (2) identification and analysis of the distinguished segmentation base.

Phase I: Analysis of the overlap

This phase concentrates on the issues of mutual association among various segmentation variables. At this stage, therefore, only the segmentation variables are used. These variables (or clusterings) were identified in the PNWRES study as GENATT, general attitudes toward economy and environment, ENRATT, energy related attitudes, BEHAVE, energy consumption behavior, PERCEPT, energy (comfortdiscomfort) perceptions, and KWHUSE, energy usage in kilowatt-hours. (Procedures used and the rationale for selection of these variables (or clusterings) are discussed in detail in the third section of this chapter). Five research questions were addressed:

\section{1) Are the segmentation variables mutually} associated? If yes, how can this mutual association be measured?

For instance, assuming that each consumer is assigned to each one of four bases independently, a multivariate 
profile for each individual results: $x_{i}=\left(x_{i 1}, x_{i 2}, x_{i 3}\right.$, $\left.x_{i 4}\right)$, where $x_{i k}$ indicates membership in segment $i$ of segmentation variable $k(k=1,2,3,4)$ (Green and Carmone, 1977). Then, the question of whether these bases are mutually interdependent is useful in determining whether one will know anything about the individual's membership in other segments when his/her membership in a set of (one or more) segments is known.

The hypothesis tested for the mutual association assessment is as follows:

$$
\begin{aligned}
& \mathrm{H}_{0}: \text { All of the variables in the system of } \\
& \text { segmentation variables are independent. } \\
& \mathrm{H}_{\mathrm{A}} \text { : Two or more of the segmentation variables are } \\
& \text { interdependent }
\end{aligned}
$$

Question \#1, in the context of PNWRES study is stated as: Are the variables GENATT, ENRATT, BEHAVE, PERCEPT, AWRRE, and KWHUSE mutually associated? I.e., do attitudes, behavior, perception, awareness, and energy use overlap, such that knowing about one of these, e.g., about a person's attitudes, would reduce one's uncertainty about another, e.g., energy use? If so, how would this association be measured using the general log-linear model versus the information theoretic method. 
2) Which basis (or set of bases) makes the highest contribution toward the mutual association?

This question addresses a method to obtain a candidate distinguished base. The answer to this question is particularly useful when the remaining segmentation variables are "accessible." One can then designate the selected variable as the distinguished segmentation variable and the remaining variables as descriptor variables.

A sample of the hypotheses tested for contributions of insividual variables to the mutual association are similar to the following:

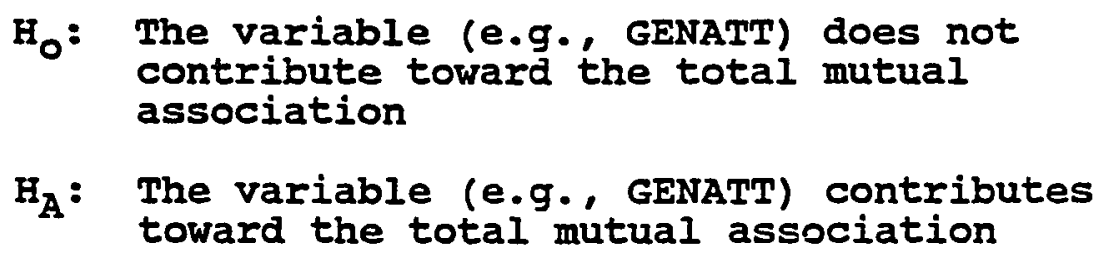

As applied to the PNWRES study, this research question becomes: Which one (or set of) variables GENATT, ENRATT, BEHAVE, PERCEPT, AWARE, and KWHUSE contributes the most toward the mutual association measured through question \#1? Such a set would then be designated as a candidate for the distinguished base. 
3) Which basis (or set of bases) makes the highest contribution toward the joint variability in the system of secmentation bases?

This question addresses a second method for identifying a candidate segmentation variable (or set of variables). In information theoretic terms, this research question differs from the previous one in that the mutual association addresses the transmission in a multivariate system, while joint variability addresses the systematic entropy.

A multivariate system may include overlapping variables for which usual tests of independence indicate statistically significant mutual association. But, since the usual association measures (e.g., chi-square, Yule's $Q$, phi-square, transmission measure, etc.) include higher levels of interaction more than once (i.e., two-way interactions are represented once, three-way interactions are represented twice, etc.), a statistically significant association measure does not necessarily indicate that a significant portion of the total system has been accounted for.

Krippendorff's "systematic entropy," which in principle measures the joint area between variables only once, will be utilized to assess how much of the uncertainty in the system is accounted for. This is the same as 
measuring the area of uncertainty shared between variables in a multivariate system.

A sample of the hypotheses tested for contributions of individual variables to the joint variability in the system of segmentation variables:
$H_{0}$ : The variable (e.g., GENATT) does not contribute toward the joint variability in the system of segmentation variables.
$H_{A}$ : The variable (e.g., GENATT) contributes toward the joint variability in the system of segmentation variables.

In the context of the PNWRES study this question investigates the contribution of each variable to the joint variability between GENATT, ENRATT, PERCEPT, AWARE, BEHAVE, and KWHUSE.

4) Knowledge of which base (or set of bases) makes the highest contribution toward the reduction of overall uncertainty in the overall system of segmentation bases?

This is a third way of identifying a candidate distinguished segmentation base. Definition of the distinguished set is analogous to von Bertalanffy's (1968) definition of the "leading part". The identification of the leading part in a multivariate system can be based on the search for the variable-set which contributes most significantly to the "systemness" of the multivariate system 
(i.e., to the mutual association, or to the joint variability in the system addressed in research questions \#2 and \#3, above), or it can be based on the set that reduces the total uncertainty in the multivariate space (research question \#4).

A sample of the hypotheses tested for contributions of individual variables to the overall uncertainty in the system of segmentation variables:

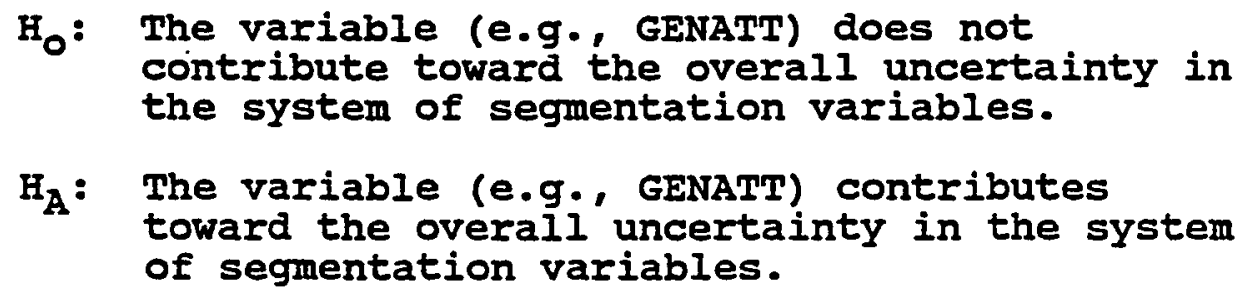

Again, in the PNWRES study this question would address the identification of a third candidate for the distinguished base. The question may be stated as: of the above variables, which one (or set) reduces the uncertainty in the system $S=$ (GENATT, ENRATT, BEHAVE, PERCEPT, AWARE, KWHUSE), the most?

A fourth way of determining the leading part is to identify the variable-set which is most central in terms of pairwise interrelationships with other variables in the system. This property is explored through research question \#5. 
5) Which variable (or set of variables) has the greatest number of statistically significant dyadic interrelationships with other variables?

This question addresses a fourth method for identifying a candidate aistinguished set. In this method the variable (or a set of variables) which interacts with a greater number of other variables is considered as being more central, and therefore, distinguished.

A sample of the hypotheses tested for the dyadic interrelationships with other variables:

$$
\begin{aligned}
& \mathrm{H}_{0}: \text { The two variables being considered are } \\
& \text { independent. } \\
& \mathrm{H}_{\mathrm{A}} \text { : The two variables are interdependent. }
\end{aligned}
$$

Phase II: Identification and Analysis of the Distinguished Variable

This phase addresses the analysis of the candidate distinguished bases identified in phase I, and selection of a best segmentation base. In this phase, the study will proceed along two distinct paths. First, the other segmentation variables are used as descriptor variables to select and analyze the distinguished base. second, a set of exogenous descriptor variables will be used to select and analyze the distinguished base.

This second approach, therefore, requires that a set of independent (descriptor) variables be identified. 
Considering that a large number of descriptor variables may be available, it is necessary to address the issues of data reduction and reinterpretation. This can be done by assessing the data in order to identify a parsimonious set of variables which best determine the segmentation variable. The descriptor variables identified for the PNWRES study include geographic/climatic environment, CLIMGEO, type of dwelling, TYPDWEL, the status of the resident in terms of renter/owner distinction, RENTOWN, the demographic cluster, DEMOG, size of the dwelling, DWELSIZE, and the level of insulation of the residence, INSUL. (A detailed discussion of selection and aggregation of variables which comprise each of the above variables (or clusterings) is given in the third section of this chapter.)

If it is assumed, for the sake of discussion, that, for example, KWHUSE, ENRATT, and BEHAVE have been identified as candidates for the distinguished base, the following four research questions are posed in this second phase of analysis: 


\section{1) Which one of the candidate distinguished}

segmentation bases, identified in phase $I$, is best

predicted once we have the knowledge of a set of relevant independent variables?

As already mentioned, once a segment (or a set of segments) is identified, the researcher needs to know how to identify and reach the individuals within this segment. It is certainly useful to know that the high electrical consumption group is comprised of individuals who, say, reside in north side, are married, have two or more children, and live in houses that were built prior to 1960, etc. The present research question addresses this issue by searching for the candidate segmentation base best predictable given a set of demographic, socioeconomic, geographic, and/or psychographic variables. (Predictability means knowing the class of the dependent variable, given the classes of the independent variables).

Therefore, in the PNWRES study the predictability is addressed as: Which base (KWHUSE, ENRATT, or BEHAVE) is best determined (i.e., accounted for) once we have the knowledge of the descriptor variable set (CLIMGEO), (TYPDWEL)，(RENTOWN)，(DEMOG)，(DWELSIZE), and (INSUL)? 


\section{2) How much of the uncertainty in the distinguished} variable is reduced when it is dichotomized into a class (or group of classes) versus all of the other classes?

It is possible to isolate the segment which is of particular interest as one class, and all other segments as another (i.e., to form a dichotomous segmentation variable, where one of the classes is composed of the "sought" group). Then, the predictability (or reduction in uncertainty) in the new variable due to the knowledge of the descriptor variables can be investigated.

In the PNWRES study, assuming that KWHUSE (containing 5 classes) is selected as the distinguished base in question \#1 of Phase II, this question may be stated as: How much of the uncertainty in the dichotomized KwHUSE is reduced with the knowledge CLIMGEU, TYPDWEL, RENTOWN, DEMOG, DWELSIZE, and INSUI under the following dichotomization schemes?

1) Classes $1 \& 2$ vs. all other classes (the lowconsumption group vs. others).

2) Class 3 vs. all other classes (the mediumconsumption group vs. others).

3) Classes $4 \& 5$ vs. all other classes (the highconsumption group vs. others).

The information obtained through these analyses is useful in assessing predictability of individual groups. 
One knows how much is known about the variable of interest given the knowledge of the descriptor variables.

3) How can one prioritize the effect of the independent variables on the distinguished set?

This question, in a way, addresses the principle of parsimony in obtaining data on the independent variables (i.e., which independent variables must be retained and which ones can be dropped). If, in prioritizing the amount of prediction made by each independent variable, it becomes evident that a variable contributes very little to predicting the dependent variable, it may be disregarded and more effort spent on obtaining data on variables with higher priority.

In the PNWRES study, under the assumption that KWHUSE is selected as the distinguished base in question \#1, Phase II: Prioritize the effect of (CLIMGEO), (TYPDWEL), (RENTOWN), (DEMOG), (DWELSIZE), and (INSUL) in determining the segmentation base KWHUSE-- i.e., which one contributes the most toward the determination of KWHUSE, which one contributes second most, etcetera?

After eliminating the descriptor variables with insignificant contribution toward the segmentation base determine the answer to the following question: 
4) What is the dependency structure in each class of the distinguished segmentation base?

In general system methodology, a structure is interpreted as "a maximally-simple, adequately-complete set of subrelations which taken together capture most or all of the constraints and relations exhibited by the system..." (Conant 1982, p.243). Dependency analysis as developed by Conant (1981, 1982) deals with a method of determining an acceptable structural model (called dependency structure) for a system taking into account the strength of relations among its variables. Later in this chapter, conant's method will be described in detail.

This research question not only addresses the construction of a structural model of the data (i.e., a diagram), but to assess the strength of the direct effects in such model. Furthermore, the subset of (statistically) significant variables in the system can be interpreted as the leading part.

Finally, in the PNWREs study one would want to: Quantify the dependency structure for each of the structural models obtained in 3 above-- i.e., given each structural model find strong and weak interrelationships. This quantification is significant due to the same reasons given for the fourth research question (i.e., gain in the 
understanding of the determinants of the criterion variable and their relationships).

Tables IV and V presents summary of the research questions and their significance in segment congruence analysis in Phases I and II, respectively. All of the research questions have significance and are of interest to marketing practitioners and theoreticians in general including those in the electrical utility industry (and other energy related fields). 
TABLE IV

RESEARCH QUESTIONS AND THEIR SIGNIFICANCE IN SEGMENT CONGRUENCE ANALYSIS (PHASE I)

\begin{tabular}{|c|c|}
\hline RESEARCH QUESTION \#1 & $\begin{array}{l}\text { Are the segmentation variables } \\
\text { mutually associated? If yes, how } \\
\text { can this mutual association be } \\
\text { measured? }\end{array}$ \\
\hline SIGNIFICANCE: & $\begin{array}{l}\text { Is it possible, by obtaining } \\
\text { information about a subset of these } \\
\text { variables, to learn about the } \\
\text { remaining variables? }\end{array}$ \\
\hline RESEARCH QUESTION \#2 & $\begin{array}{l}\text { Which basis (or set of bases) makes } \\
\text { the highest contribution toward the } \\
\text { mutual association? }\end{array}$ \\
\hline SIGNIFICANCE: & $\begin{array}{l}\text { Identifying a candidate for the } \\
\text { distinguished segmentation set } \\
\text { (Method 1). }\end{array}$ \\
\hline RESEARCH OUESTION \#3 & $\begin{array}{l}\text { Which basis (or set of bases) makes } \\
\text { the highest contribution toward the } \\
\text { joint variability in the system of } \\
\text { segmentation bases? }\end{array}$ \\
\hline SIGNIFICANCE: & $\begin{array}{l}\text { Identifying a candidate for the } \\
\text { distinguished segmentation set } \\
\text { (Method 2). }\end{array}$ \\
\hline RESEARCH OUESTION \#4 & $\begin{array}{l}\text { Knowledge of which base (or set of } \\
\text { bases) makes the highest } \\
\text { contribution toward the reduction } \\
\text { of overall uncertainty in the } \\
\text { overall system of segmentation } \\
\text { bases? }\end{array}$ \\
\hline SIGNIFICANCE: & $\begin{array}{l}\text { Identifying a candidate for the } \\
\text { "distinguished" segmentation set } \\
\text { (Method 3). }\end{array}$ \\
\hline RESEARCH OUESTION \#5 & $\begin{array}{l}\text { Which variable (or set of } \\
\text { variables) has the greatest number } \\
\text { of statistically significant dyadic } \\
\text { interrelationships with other } \\
\text { variables? }\end{array}$ \\
\hline SIGNIFICANCE: & $\begin{array}{l}\text { Identifying a candidate for the } \\
\text { "distinguished" segmentation set } \\
\text { (Method 4). }\end{array}$ \\
\hline
\end{tabular}


TABLE V

RESEARCH QUESTIONS AND THEIR SIGNIFICANCE IN SEGMENT CONGRUENCE ANALYSIS (PHASE II)

\begin{tabular}{|c|c|}
\hline RESEARCH QUESTION \#I & $\begin{array}{l}\text { Which one of the candidate } \\
\text { distinguished segmentation bases, } \\
\text { identified in phase I, is best } \\
\text { predicted once we have the } \\
\text { knowledge of a set of relevant } \\
\text { independent variables? }\end{array}$ \\
\hline SIGNIFICANCE: & $\begin{array}{l}\text { To select a "distinguished" } \\
\text { segmentation basis (or a set of } \\
\text { bases) amongst the candidates } \\
\text { obtained in questions } 2,3,4 \text {, and } \\
5 \text { of Phase } I \text {. }\end{array}$ \\
\hline RESEARCH QUESTION \#2 & $\begin{array}{l}\text { How much of the uncertainty in the } \\
\text { distinguished variable is reduced } \\
\text { when it is dichotomized into a } \\
\text { class (or group of classes) versus } \\
\text { all of the other classes? }\end{array}$ \\
\hline SIGNIFICANCE: & $\begin{array}{l}\text { This will show which class (or } \\
\text { group of classes) of the } \\
\text { distinguished variable is best } \\
\text { represented by the independent } \\
\text { variables in the system. }\end{array}$ \\
\hline RESEARCH QUESTION \#3 & $\begin{array}{l}\text { How can one prioritize the effect } \\
\text { of the independent variables on the } \\
\text { distinguished set? }\end{array}$ \\
\hline SIGNIFICANCE : & $\begin{array}{l}\text { To rank order the contributions of } \\
\text { the independent variables to } \\
\text { explaining (reducing uncertainty } \\
\text { in) each dependent variable. }\end{array}$ \\
\hline RESEARCH QUESTION \#4 & $\begin{array}{l}\text { What is the dependency structure in } \\
\text { each class of the distinguished } \\
\text { segmentation base? }\end{array}$ \\
\hline SIGNIFICANCE: & $\begin{array}{l}\text { What is the ordinality of } \\
\text { relationships among variables? }\end{array}$ \\
\hline
\end{tabular}




\section{METHODOLOGY}

This section includes four parts. The first part describes the information theoretic expressions used in assessing each of the research questions. The second part entails a brief description of the computer programs used to perform the information theoretic analysis. The third part describes, where applicable, the log-linear and logit models used to assess the research questions. And finally, the fourth part discusses the reasons for using the information theoretic methodology as compared with the more commonly used $\log -1$ inear and logit models.

\section{Information Theoretic Methodology}

As mentioned earlier, Appendix II introduces the basic concepts in information theory and its extension to analysis of dependence and independence in a multivariate system and structural modeling. Information theoretic methods can be used to address the research questions introduced in the previous part.

Phase I: Analysis of the Segmentation Variables. This phase addresses the assessment of mutual association among the segmentation variables, and then, discusses four methods of identifying candidate segmentation variables. 
Research Question 1. Information theoretic entropy transmission can be used to assess interrelationships among a number of segmentation variables. To measure the degree of association among variables $A, B, C$, and $D$, the transmission measure, $T(A: B: C: D)$, is computed using equations (7) and (8):

(7) $H(A, B, C, D)=-\Sigma_{i} \Sigma_{j} \Sigma_{k} \Sigma_{1} P\left(A_{i}, B_{j}, C_{k}, D_{I}\right) \log _{2} P\left(A_{i}, B_{j}, C_{k}, D_{l}\right)$

(8) $T(A: B: C: D)=H(A)+H(B)+H(C)+H(D)-H(A, B, C, D)$

where $i=1,2, \ldots, n_{A}, j=1,2, \ldots, n_{B}, k=1,2, \ldots, n_{C}$
$l=1,2, \ldots, n_{D^{\prime}}$ and $n_{A}, n_{B}{ }^{n} n^{\prime}$ and $n_{D}$ are numbers of
classes of variables $A,{ }_{B},{ }^{\prime}$, and $D^{\prime}$ respectively.

This addresses the first research question in phase I (i.e., the mutual association among the segmentation bases). Transmission in a multivariate system measures the amount of overlap between the uncertainties of the variables. Additionally, it measures this overlap with respect to the ordinalities of the overlaps: Those parts that overlap once (i.e., demonstrate two-way interactions) are represented once, those with three-way interactions are represented twice, those with four-way interactions are represented three times, etc.

Figure 3 demonstrates this property spatially. In terms of the areas numbered in the venn diagram in Figure 3, transmission is represented as: 
$T(A: B: C: D:)=H(A)+H(B)+H(C)+H(D)-H(A, B, C, D)$

$(2,4,5,5,6,7,7,8,8,8,9,9,10,11,11,12)=$

$(1,2,4,5,7,8,10,11)+(2,3,5,6,8,9,11,12)+$

$(7,8,9,10,11,12,13,15)+(4,5,6,7,8,9,13,14)-$

$(1,2,3,4,5,6,7,8,9,10,11,12,13,14,15)$.

Note that overlapping areas $2,4,6,10,12$, and 13

are included once in the total transmission; 5, 7, 9, and 11 are included twice, and 8 is included three times.

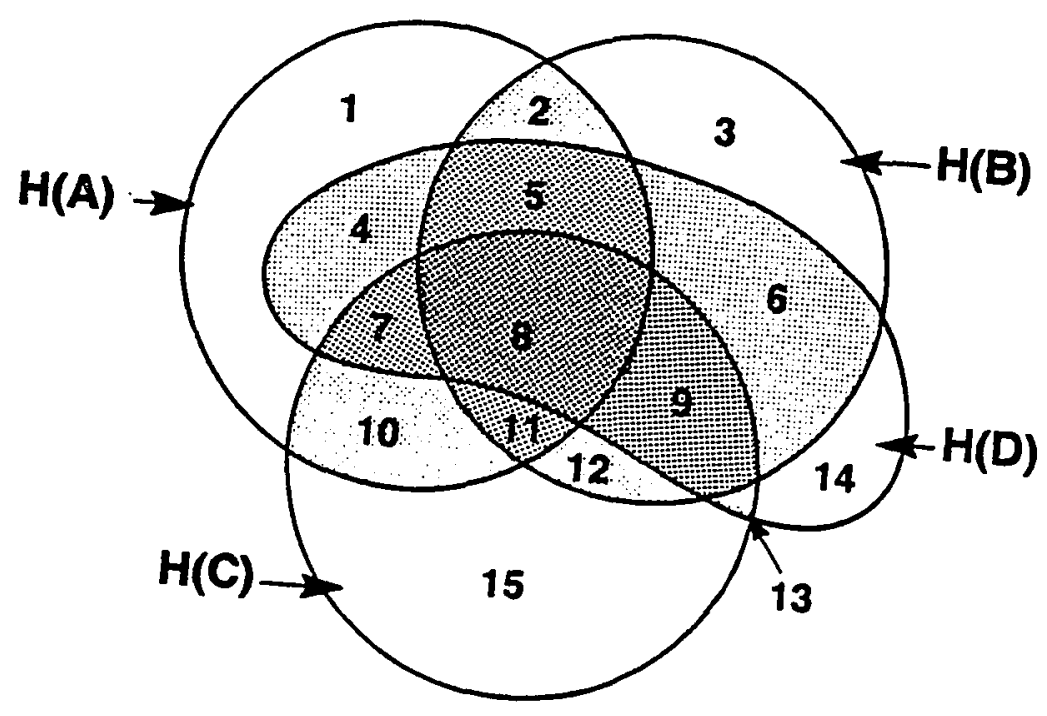

Figure 3. Spațial representation of transmission.

As previously mentioned, transmission measure approximates a chi-square distribution (Attneave, 1959) with the likelihood ratio chi-square equal to $2 . \log (2) \cdot N . T$. The degrees of freedom are computed based on the formula given 
by Broekstra (198I) and reproduced in Chapter II (see Page 43).

Transmission-based measures of association have been used widely in general system methodology (Broekstra, van Bueren, etc.). A normalized measure of transmission can be obtained by computing the ratio as shown in Equation (9):

(9) $T_{\text {NORM }}=\frac{T}{T_{\max }}=\frac{T}{\sum_{i} H\left(X_{i}\right)-\operatorname{Max}\left(H\left(X_{i}\right)\right\}}$

This measure has a minimum of zero and a maximum of $I$. These are desired properties in evaluating measures of association (Blalock 1972).

Research question $\# 2$. The second research question in phase I (i.e., which variable (or set) contributes the most toward the total mutual association) is addressed by computing the differences between the overall association, $T(A: B: C: D)$, and each of the conditional associations, $T_{A}(B C D), T_{B}(A C D), T_{C}(A B D), T_{D}(A B C), T_{A B}(C D), T_{A C}(B D)$, $T_{A D}(B C), T_{B C}(A D), T_{B D}(A C)$, and $T_{C D}(A B)$. Equation (10) calculates the transmission difference $\left(\Phi_{i} T\right)$ :

$$
\begin{aligned}
\Phi_{1} T & =T(A: B: C: D)-T_{A}(B: C: D) \\
\Phi_{2} T & =T(A: B: C: D)-T_{B}(A: C: D) \\
\Phi_{3} & =T(A: B: C: D)-T_{C}(A: B: D) \\
\Phi_{4} & =T(A: B: C: D)-T_{D}(A: B: C) \\
\Phi_{5} T & =T(A: B: C: D)-T_{A B}(C: D) \\
\Phi & T=T(A: B: C: D)-T_{A C}(B: D) \\
\text { etC. } &
\end{aligned}
$$


The largest difference would identify the variable with the highest contribution to the mutual association (or equivalently, with the lowest remaining association among the unknown variables). The four-variable system in Figure 4 will be used to demonstrate questions 2,3 , and 4 in phase I, graphically:

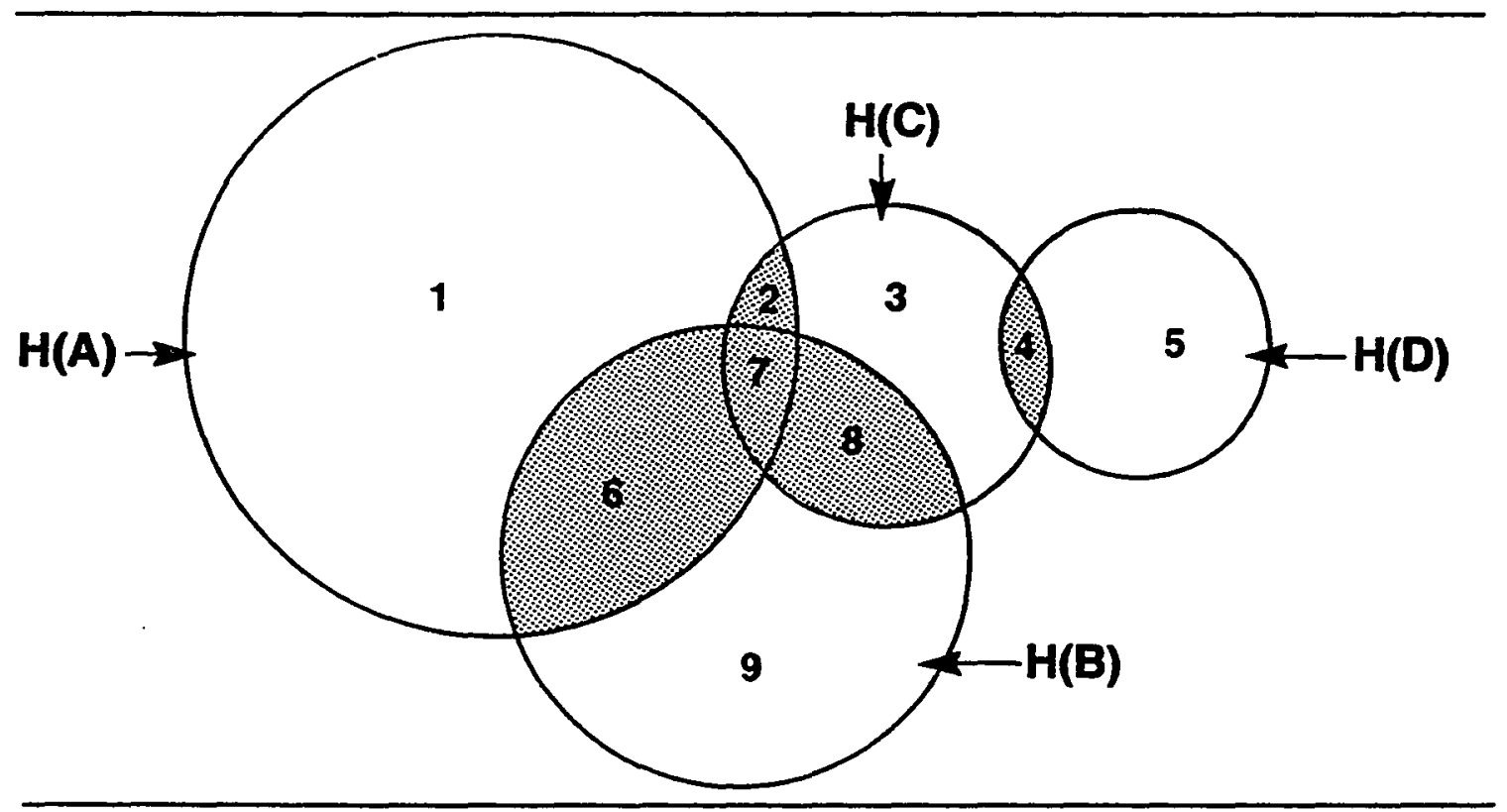

Figure 4. A System of four variables.

Variable B clearly includes most of the mutual association (as measured by transmission) among these four variables. The total mutual association is composed of areas $2,4,6,2 * 7$, and 8 . Variable $B$ accounts for 6, 7, and 8 which is the highest amount as compared to 2,6 , and 7 
accounted for by $A, 2,7$, and 8 accounted for by $C$, and 4 accounted for by $D$.

A significance test can be performed on any of the equations in (3). Here, the null hypothesis will be stated as, e.g., $T_{A}$ (B:C:D) is not consistent with the independence model for $(B, C, D)$ subtables within each class of $A$ (or, equivalently, $T(A: B: C: D)-T_{A}(B: C: D)$ fits the data). Then, small values of chi-square would reject the null hypothesis (or large values of the alternative model would reject its null hypothesis) and establish significance for the given variable. This is because if much of the mutual association is due to the known variable, then, holding it constant would render the rest of the variables independent (i.e., non-communicating, in information theoretic terms)

Alternately, partial-normalized transmissions or partial covariability coefficients can be used to measure the changes in the overlap given each variable. Here, then, a threshold criterion (e.g., 20\%) would be established to judge these measures with.

Research Question \#3. The previous research question addressed the contribution of each segmentation variable to the total mutual association among the segmentation variables. The third research question assesses this contribution to the overall joint variability among these variables. 
To calculate the joint variability in a system Krippendorff's measure of systematic entropy will be used. systematic entropy is computed by subtracting the noise factor for each variable from the total uncertainty in the system:

$$
S(W: X: Y: Z)=H(W, X, Y, Z)-H_{X Y Z}(W)-H_{W Y Z}(X)-H_{W X Z}(Y)-H_{W X Y}(Z)
$$

This calculation, as shown in Equation (11), in effect excludes the uncertainty in each variable which is not accounted for by any other variable (i.e., the noise). What remains is a "one-layer" measurement of the overlap in the system. Figure 5 depicts a four-variable system. The shaded area is the systematic entropy of this system.

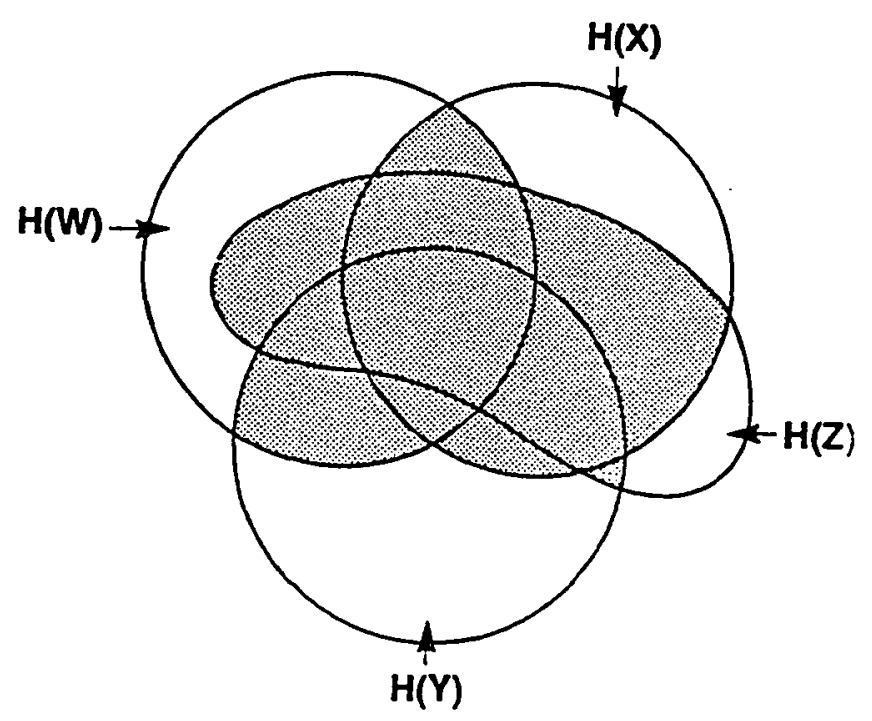

Eigure 5. Systematic entropy of a four-variable system. 
Partial systematic entropies can then be used to assess the contribution of each variable to the joint variability in the system. The information theoretic equations for the four-variable case developed below:

$$
\begin{aligned}
& \Phi_{1} S=S(A: B: C: D)-S_{A}(B: C: D) \\
& \Phi_{2} S=S(A: B: C: D)-S_{B}(A: C: D) \\
& \Phi_{3} S=S(A: B: C: D)-S_{C}(A: B: D) \\
& \Phi_{4} S=S(A: B: C: D)-S_{D}(A: B: C) \\
& \Phi_{5} S=S(A: B: C: D)-S_{A B}(B: C) \\
& \Phi_{6} S=S(A: B: C: D)-S_{A C}(B: D)
\end{aligned}
$$

etc.

Interestingly, the above equations are identical to difference between ( $n$ )-variable and (n-k)-variable transmissions, where, $n$ refers to the first term in the above equations (e.g., $A: B: C: D)$, and $n-k$ refers to the unknown portion of the second term (e.g., B:C:D). The proof for this assertion follows:

The three variable case

$$
\begin{aligned}
S(A B C)-S_{A}(B C)= & H(A B C)-H_{B C}(A)-H_{A C}(B)-H_{A B}(C) \\
& -\left(H_{A}(B C)-H_{A C}(B)-H_{A B}(C)\right) \\
= & H(A B C)-H_{B C}(A)-H_{A}(B C) \\
= & H(A B C)-H(A B C)+H(B C)-H(A B C)+H(A) \\
= & -H(A B C)+H(B C)+H(A) \\
= & H(A)+H(B)+H(C)-H(A B C)-H(B)-H(C)+H(B C) \\
= & {[H(A)+H(B)+H(C)-H(A B C)] } \\
& -[H(B)+H(C)-H(B C) \\
= & T(A B C)-T(A B)
\end{aligned}
$$


In General

$$
\begin{aligned}
& S(A B C \ldots)-S_{A}(B C \ldots) \\
&= H(A B C \ldots)-H_{B C} \ldots(A)-H_{A C} \ldots(B)-H_{A B} \ldots(C)-\ldots \\
&-\left(H_{A}(B C \ldots)-H_{A C} \ldots(B)-H A B \ldots(C)-\ldots g\right. \\
&= H(A B C \ldots)-H_{B C} \ldots(A)-H_{A}(B C \ldots) \\
&=H(A B C \ldots)-H(A B C \ldots)+H(B C \ldots)-H(A B C \ldots)+H(A) \\
&=-H(A B C \ldots)+H(B C \ldots)+H(A) \\
&=H(A)+H(B)+H(C)+\ldots-H(A B C \ldots)-H(B)-H(C)-\ldots+H(B C \ldots) \\
&=[H(A)+H(B)+H(C)+\ldots \ldots-H(A B C \ldots)] \\
&-[H(B)+H(C)+\ldots-H(B C \ldots)] \\
&= T(A B C \ldots)-T(B C \ldots)
\end{aligned}
$$

Therefore, a significance test can easily be performed (e.g., the null hypothesis is $T(A: B: C: D)-T(B: C: D)$ adequately represents the system, i.e., no significant difference between the expected frequencies generated through products of marginals and the observed values, resulting in a statistically insignificant (or small) likelihood-ratio chisquare value). Here, obviously, sufficiently large values of chi-square would result in the rejection of the null hypotheses.

Again, a normalized measure may be developed which would reveal the amount, rather than just the significance, of these measures. This measure is $1-s_{i} / S$, where $S$ is the total systematic entropy in the system and $s_{i}$ is the systematic entropy remaining once variable $i$ is known. This measure is equal to zero only if knowing $i$ does not reduce 
the systematic entropy, and it is equal to one if $i$ accounts for all of the systematic entropy.

Research Question \#4. The fourth research question in phase I (i.e., which variable(s) contributes the most toward the total uncertainty in the system of segmentation bases) is assessed using a similar approach as that used for the second question; however, here, instead of transmission differences, uncertainty differences are computed using equation (7). This is shown in the set of equations in (13) below:

$$
\begin{aligned}
& \Phi_{1} H=H(A, B, C, D)-H(A) \\
& \Phi_{2} H=H(A, B, C, D)-H(B) \\
& \Phi_{3} H=H(A, B, C, D)-H(C) \\
& \Phi_{4} H=H(A, B, C, D)-H(D) \\
& \Phi_{5} H=H(A, B, C, D)-H(A B) \\
& \Phi_{6} H=H(A, B, C, D)-H(A C)
\end{aligned}
$$

etc.

In Figure 4, judging spatially, variable A accounts for most of the area of uncertainty occupied by the fourvariable system. Therefore, knowledge of variable A would reduce the uncertainty about the total system by the most amount

Research Question \#5. In order to assess the fifth research question in phase I (i.e., which variable has the greatest number of significant dyadic transmissions with the remaining variables) a series of two-way transmission tables are utilized in the following form: 


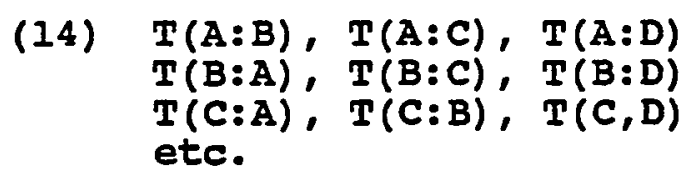

Then, a likelihood-ratio chi-square test of significance is performed for each transmission value in the table and non-significant transmissions are eliminated. Finally, the variable with the greatest number of surviving transmissions with other variables is identified as the candidate for the distinguished base.

Again, referring to Figure 4 , variable $c$ has the greatest number of dyadic relations within the system (i.e., it has interrelations with $A, B$, and D), While $A$ and $C$ have two such relations and D has only one. By this criterion, variable $c$, therefore, would be most central in the fourvariable system.

Phase II: Selection and Analysis of the Distinguished Segmentation Variable. This phase first selects a distinguished segmentation variable (or set of variables), then, proceeds with analyzing it in detail. So far, variables $A, B, C$, and $D$ have been used to exemplify the segmentation variables. In order to continue with this example, variables $W, X, Y$, and $Z$ will by introduced to exemplify exogenous descriptor variables. 
The actual analyses will first use the other segmentation variables as descriptor variables, and next the analyses are repeated with the exogenous variables. Here, however, only the exogenous variables are used for the discussion of the methodological issues, as the two sets of analyses are identical with respect to the methods used.

Research Question \#I. In order to address the first research question in phase II (i.e., which segmentation basis is best determined once we have knowledge of a set of relevant descriptor variables?), the variability (entropy) in each candidate distinguished segmentation base, obtained in phase $I$, can be decomposed into the portion determined by the descriptor variables and the portion which remains unexplained. The expression, $\mathrm{H}_{X Y Z}(A)[=H(A X Y Z)-H(X Y Z)]$ is interpreted as variability in $A$ unaccounted for by variables $X, Y$, and $Z$. The descriptor variables $X, Y, Z$, etc. can be selected either from the set of segmentation variables or other (exogenous) variables. Similar expressions are then obtained for other candidate segmentation variables: .

$$
\begin{aligned}
& \Phi H(A)=H(A)-H_{X Y Z}(A) \\
& \Phi H(B)=H(B)-H_{X Y Z}(B) \\
& \Phi H(C)=H(C)-H_{X Y Z}(C) \\
& \Phi H(D)=H(D)-H_{X X Z}(D) \\
& \Phi H(A B)=H(A B)-H_{X Y Z}(A B) \\
& \Phi H(A C)=H(A C)-H_{X Y Z}(A C) \\
& \text { etc. }
\end{aligned}
$$


Equation (15) computes the reduction in uncertainty of the segmentation variable due to the descriptor variables $x$, $\mathrm{Y}$, and $\mathrm{Z}$. The segmentation variable with the greatest reduction in its uncertainty is then said to be determined "best" by these descriptor variables. This variable can then be designated as the "distinguished" variable.

Figure 6 presents $\mathrm{H}_{\mathrm{XYZ}}(\mathrm{A})$ graphically. The shaded portion of the area of uncertainty for $A$ is the portion represented by the (descriptor) variables $X, Y$, and $Z$.

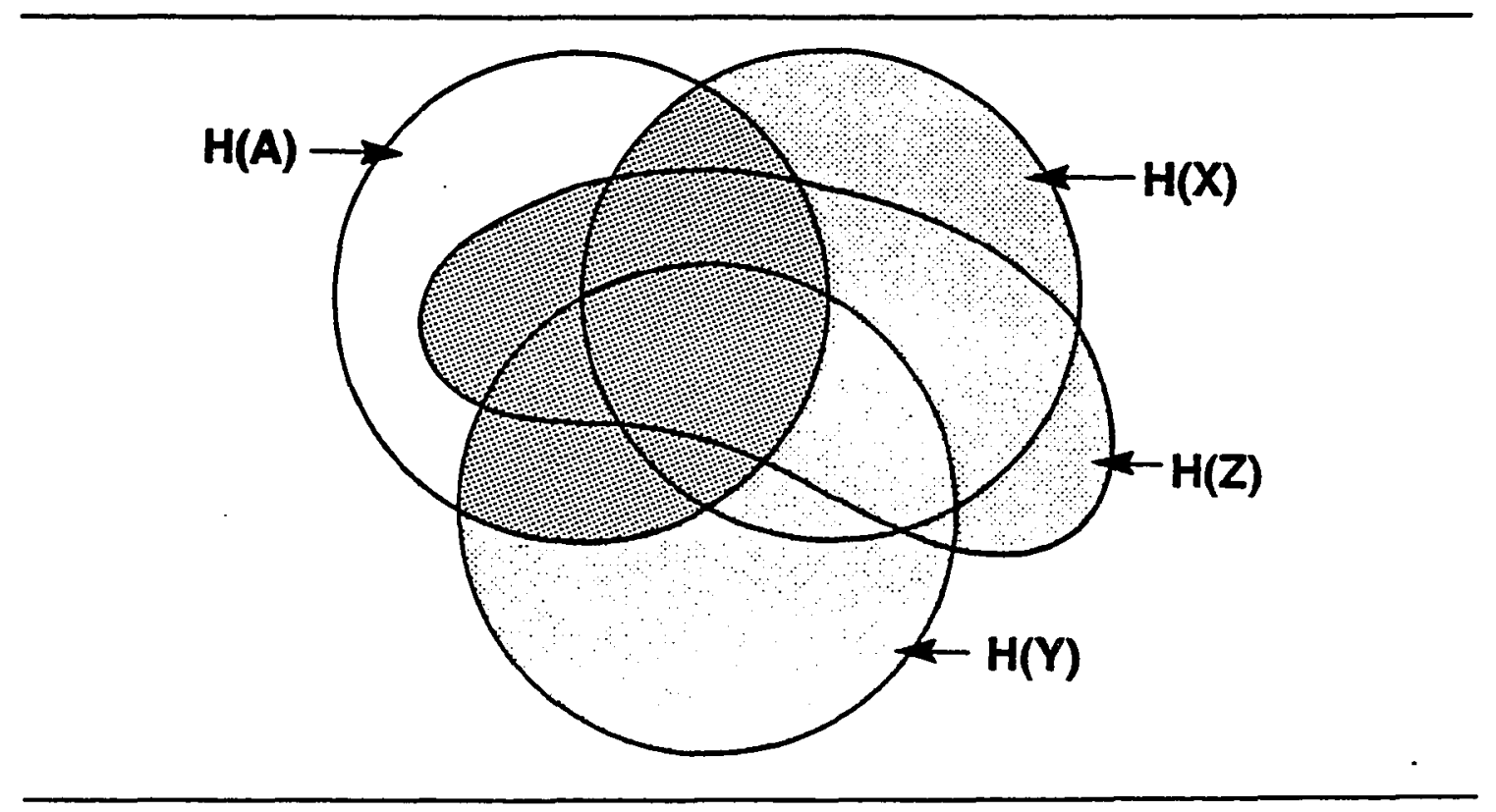

Figure 6. Determination of variable $A$ with the knowledge of the descriptor variables $X, Y$, and $\mathbf{Z}$.

As an example of how this method works, consider the case where the other segmentation variables are used as 
descriptors. Therefore, if, for example, variable $A$ is under investigation as a potential distinguished variable, B, C, and D are considered the descriptor variables. For instance, considering variables $A, C$, and D in Figure 4; it is evident that a greater proportion (or percent) of the uncertainty in $C$ is accounted for with the knowledge of the other variables, followed by $A$ and $D$. Visually, the following relationship between the proportions of uncertainties accounted for seems to exist for these three variables:

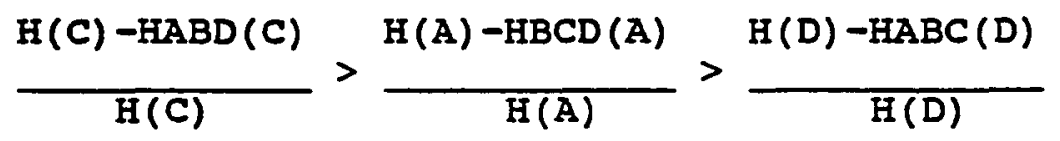

or, as represented by the areas in figure 4:

$(2,4,7,8) /(2,3,4,7,8)>(2,6,7) /(1,2,6,7)>$ $4 /(4,5)$.

Research Question \#2. This research question uses the algorithm developed for the first research question in this phase to assess the predictability (or reduction in uncertainty) of the distinguished segmentation variable under various collapsing schemes. The criteria for aggregation of classes (i.e., collapsing schemes) are subjective and based on the needs of the decision maker.

In terms of electricity consumption one may collapse the "very low," "low", and "medium," electricity consumption 
groups as the first class, and high and very high groups as the second class. Here, the predictability of each of electricity consumption can be assessed in a dichotomized variable which is easy to interpret.

Research Question \#3. The third question in Phase II (i.e., prioritizing the effect of the independent variables on the distinguished set) can be assessed using a method proposed by Ashby (1964) and used by Abrahamse and van Bueren (1980) and Krippendorff (1981). In this method, first marginal entropy (marginal variability) is considered for the variable of interest (e.g., A), then, bivariate transmissions are computed between the segmentation variable and the descriptor variables (i.e., $T(A: W), T(A: X), T(A: Y)$, $T(A: Z))$. These bivariate transmissions are in turn used to identify the descriptor variable that contributes the most to the segmentation variable. Then conditional bivariate transmissions are computed using the variable obtained above -- i.e., assuming $\mathrm{X}$ is selected as the variable with the highest bivariate transmission with $A, T_{X}(A: Y)$ and $T_{X}(A: Z)$ are computed, and the largest of these is selected. Repeating this process for all of the independent variables will result in some partitioning similar to the following:

$$
\text { (17) } H(A)=T(A: X)+T_{X}(A: Y)+T_{X Y}(A: Z)+H_{X Y Z}(A)
$$


where, $\mathrm{x}$ is the variable with the highest bivariate transmission with $A ; Y$ is the variable with the highest bivariate transmission with $A$, given $X ; z$ is the variable with the highest bivariate transmissios with $A$, given $X$ and $\mathrm{Y}$; and $\mathrm{H}_{\mathrm{XYZ}}(\mathrm{A})$ is the remaining variability in $\mathrm{A}$ not explained by variables $\mathrm{X}, \mathrm{Y}$, or $\mathrm{Z}$. Therefore, we can assert that: of the variability in variable $A$, the amount equivalent to $T(A: X), T_{X}(A: Y)$, and $T_{X Y}(A: Z)$ are explained by variables $X, Y$, and $Z$, respectively, while $\mathrm{H}_{X Y Z}(A)$ is exclusive to variable $A$.

Figure 7 illustrates this situation. Variable $A$ is assumed to be the segmentation variable. Again, judging spatially, variable $\mathrm{z}$ seems to be the highest contributor as $(4,5,11)$ occupies the largest area of uncertainty as compared to $W($ i.e., $3,4,8), X$ (i.e., 8,9) and $Y$ (i.e., 10, 11). It is also apparent that $w$ is the next highest contributor as $(3,8)$ (i.e., $\mathrm{T}_{\mathrm{Z}}(\mathrm{A}: \mathrm{W})$ ) is larger than $(10)$ (i.e., $T_{Z}(A: Y)$ ) and $T_{Z}(A: Y)=(8,9)$. By the same token, $Y$ and $\mathrm{x}$ are next in the ranking, respectively.

Research Question \#4. The fourth question in phase II is stated as: What is the dependency structure in each class of the distinguished segmentation base? This question can be approached by utilizing the technique for "dependency analysis" suggested by conant (1982). 


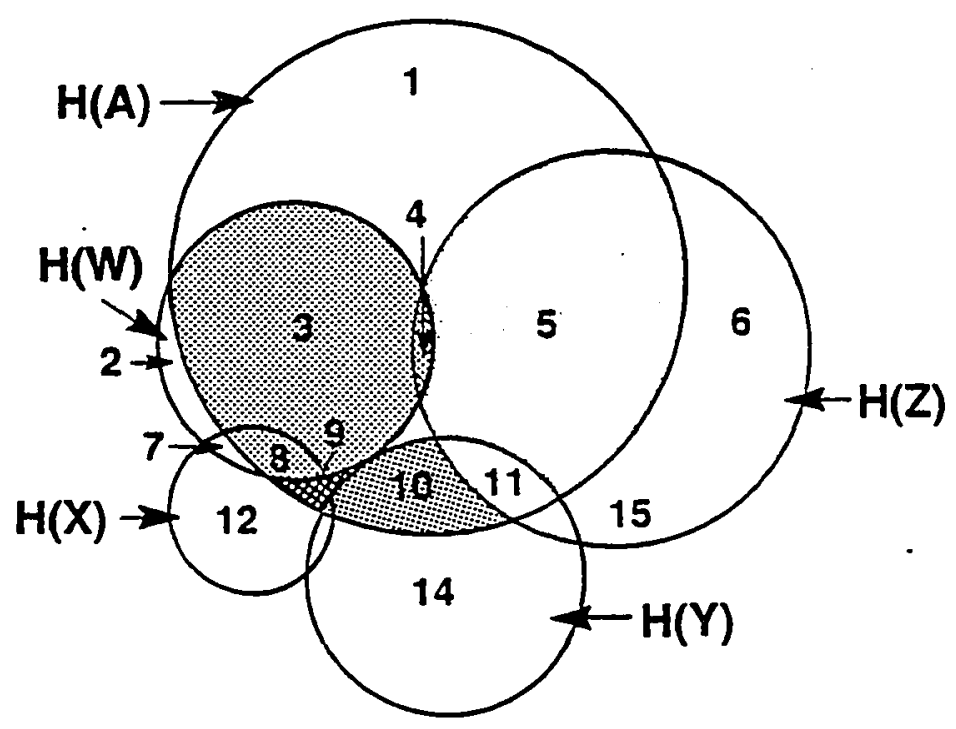

Figure 7. Prioritization of the contributions of variables $W, X, Y$, and $Z$ to determine variable $A$.

In dependency analysis, first bivariate transmission between each variable $(e . g ., w)$ and the remaining variables in the system is computed. Then the single variable with the highest transmission is identified.--i.e., $\operatorname{Max}(T(W: X)$, $T(W: Y), T(W: Z)\}$. Next, bivariate transmissions between the variable under consideration (i.e., w) and two-variable combinations, taken as a whole, of other variables (i.e., $X Y, X Z, Y Z$ ) are computed in order to determine the twovariable component that has the highest transmission with the variable under investigation --i.e., $\operatorname{Max}(T(W: X Y)$, $T(W: X Z), T(W: Y Z)\}$. Next, bivariate transmissions between the variable under investigation (i.e., w) and three- 
variable combinations, taken as a whole, of the remaining variables are computed such that the maximum is identified (note that, here, there is only one three-variable combination.

Next, another variable is considered (e.g., $\mathrm{x}$ ). In the same manner as above, then, the highest bivariate transmissions with single, double, triple, etc. variables are identified. subsequently, a table similar to Table VI is constructed.

In Table VI, column 1 is the variable under consideration, column 2 is the number of variables in the combination $s_{i}$, column 3 is the $s_{i}$ which has the highest bivariate transmission with the variable in column 1 , column 4 lists bivariate transmissions between the variables in column 1 and 3 , the latter taken as an aggregate variable. Column 5 is column 4 divided by the entropy of the variable in column 1, column 6 is column 5 as a percent of $\operatorname{Max}(T / H(i))$, and Column 7 is the incremental effect of variables for each group measured by the transmission differences.

This table is in turn used to produce graphs of each variable and other variables showing the strength of relationships. 
TABLE VI

CALCULATIONS FOR DEPENDENCY ANALYSIS OF WXYZ

\begin{tabular}{|c|c|c|c|c|c|c|}
\hline (1) & (2) & (3) & (4) & (5) & $(6)$ & (7) \\
\hline$i$ & $\mathbf{n}$ & $s_{i}$ & $T\left(i: S_{i}\right)$ & $T_{n} / H(i)$ & $\%$ & $T(n)-T(n-1)$ \\
\hline $\bar{W}$ & $\begin{array}{l}1 \\
2 \\
3\end{array}$ & $\begin{array}{l}X \\
X Y \\
X Y Z\end{array}$ & $\begin{array}{l}T(W: X) \\
T(W: X Y) \\
T(W: X Y Z)\end{array}$ & $\begin{array}{l}\overline{\mathrm{T}_{1} / \mathrm{H}(\mathrm{W})} \\
\mathrm{T}_{2} / \mathrm{H}(\mathrm{W}) \\
\mathrm{T}_{3} / \mathrm{H}(\mathrm{W})\end{array}$ & $\begin{array}{l}\star \\
* \\
*\end{array}$ & $\begin{array}{l}\overline{T(W: X)} \\
T(W: X Y)-T(W: X) \\
T(W: X Y Z)-T(W: X Y)\end{array}$ \\
\hline$x$ & $\begin{array}{l}1 \\
2 \\
3\end{array}$ & $\begin{array}{l}\text { W } \\
\text { WY } \\
\text { WYZ }\end{array}$ & $\begin{array}{l}T(X: W) \\
T(X: W Y) \\
T(X: W Y Z)\end{array}$ & $\begin{array}{l}T_{I} / H(X) \\
T_{2} / H(X) \\
T_{3} / H(X)\end{array}$ & $\begin{array}{l}* \\
* \\
*\end{array}$ & $\begin{array}{l}T(X: W) \\
T(X: W Y)-T(X: W) \\
T(X: W Y Z)-T(X: W Y)\end{array}$ \\
\hline$Y$ & $\begin{array}{l}1 \\
2 \\
3\end{array}$ & $\begin{array}{l}\mathbf{X} \\
\mathrm{XW} \\
\mathrm{XWWZ}\end{array}$ & $\begin{array}{l}T(Y: X) \\
T(Y: X W) \\
T(Y: X W Z)\end{array}$ & $\begin{array}{l}\mathrm{T}_{1} / \mathrm{H}(\mathrm{Y}) \\
\mathrm{T}_{2} / \mathrm{H}(\mathrm{Y}) \\
\mathrm{T}_{3} / \mathrm{H}(\mathrm{Y})\end{array}$ & $\begin{array}{l}* \\
* \\
*\end{array}$ & $\begin{array}{l}T(Y: X) \\
T(Y: X W)-T(Y: X) \\
T(Y: X W Z)-T(Y: X W)\end{array}$ \\
\hline $\mathbf{Z}$ & $\begin{array}{l}1 \\
2 \\
3\end{array}$ & $\begin{array}{l}X \\
X Y \\
X Y W\end{array}$ & $\begin{array}{l}T(Z: X) \\
T(Z: X Y) \\
T(Z: X Y W)\end{array}$ & $\begin{array}{l}\mathrm{T}_{1} / \mathrm{H}(\mathrm{Z}) \\
\mathrm{T}_{2} / \mathrm{H}(\mathrm{Z}) \\
\mathrm{T}_{3} / \mathrm{H}(\mathrm{Z})\end{array}$ & $\begin{array}{l}* \\
* \\
*\end{array}$ & $\begin{array}{l}T(Z: X) \\
T(Z: X Y)-T(Z: X) \\
T(Z: X Y W)-T(Z: X Y)\end{array}$ \\
\hline
\end{tabular}

* $T\left(i: S_{i}\right)$ as a percent of the $\max \left(T\left(i: S_{i}\right)\right)$ in the block $i$.

In order to construct such graphs, Conant (1981, 1982) suggests that a threshold level be selected based on which components of the dependency structure are identified. For instance, a threshold level of $80 \%$ would suggest that for each variable $i$ (Column 1 ) in Table VI level $n$ (Column 2) is selected such that the $\frac{o}{\gamma}($ Column 6 ) is at most (to be explained shortly) 80\%. The level $\Omega$, as selected in the above manner would, then, determine what other variables are included in the component which includes variable $i$. 
The above procedure would ensure that variables included in a component include at most $80 \%$ of the transmission between variable $i$ and the remaining variables. - Then, the variables suggested by level $n$ for each $i$ are used to form graphs of each component. For instance, let us assume that $n=2$ for $i=w$. This indicates that variables $x$ and $Y$ should be included in that component. Further, suppose that $T(n)-T(n-1)$ (Column 7 ) for the above $X$ and $Y$ are 0.4 and 0.1 , respectively. This would suggest that the first component on the dependency structure be WXY, with a graph in which the width of the line indicating the relation between $W$ and $X$, and $W$ and $Y$, represented such that the contributions of the variables $X$ and $Y$. to the transmission $T(W: X Y)$ are reflected.

In order to ensure that the sequence of inclusion of variables in each component (i.e., which variable was selected first, second, etc.) is reflected, they are numbered (as 1, 2, etc.) on the graph of each component. This will indicate that the contribution of a variable selected as number two assumes the inclusion of the first variable (i.e., it shows the incremental contribution to the variable i), and so on. 
Finally, after all of the components are selected and their graphs are constructed, a combined graph is discerned from the individual graphs. It should be noted that, often, the width of the line between two variables determined by two different levels of $i$ do not coincide (i.e., they do not show the same amount of contribution). The reason for this is the fact that the two width show incremental effects based on two separate conditions (i.e., preceding variables) In these cases, Conant selected the wider line, a convention which is followed here. Hypothetical graphs of the four components shown in Table VI and the final graph which emerges from them are illustrated in figure 8.

(a)

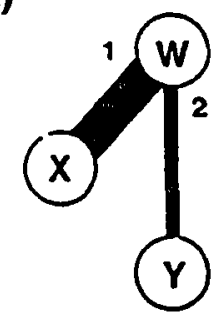

(b)

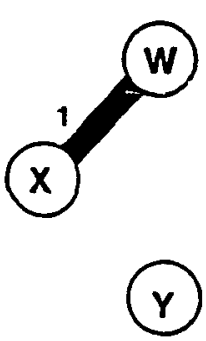

(e)

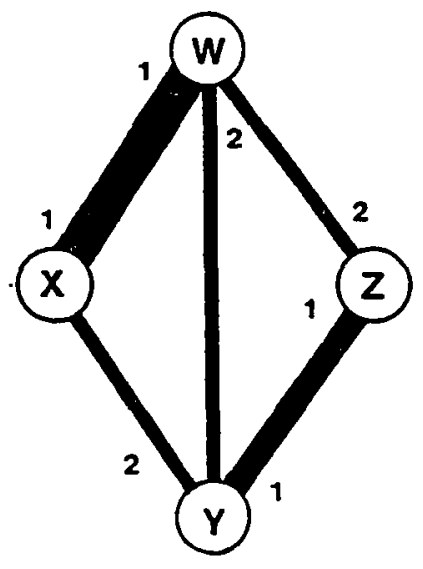

(d)

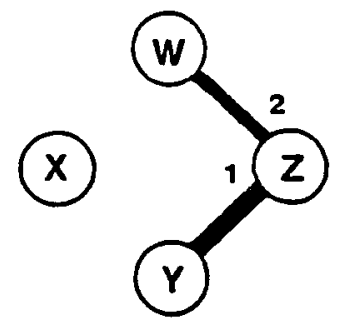

(c)

(w)

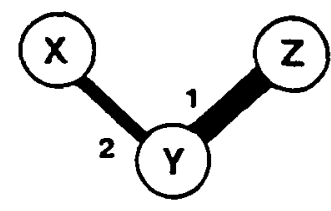

Figure 8. A sample dependency analysis diagram. 
In order to discern the structural model (in the traditional sense, e.g., WXY/XZ/YZ) the following procedure is used. First, all components are included. Thus, based on Figure 8 , WXY/WX/WYZ/XYZ is obtained. Then, all of the embedded elements (i.e., components included in another component) as well as all but one of the identical elements are removed. This will produce $W X Y / W X Z / X Y Z$ in the above example. This will produce a structure of the data which is an approximation of the optimal structural model ( Broekstra 1981, Conant 1981, 1982).

\section{Computer Program}

A computer program was developed to perform the information theoretic analyses. SYSENT is a FORTRAN program, available in both mainframe and microcomputer formats. It performs calculations of entropies, transmissions, and systematic entropies for an arbitrary number of variables (currently set at 10) and an arbitrary number of classes for each variable (currently set at 10). SYSENT takes as input NDIM, the number of variables in the system, IDIM(i), $i=1,2, \ldots, N D I M$, number of classes per variable, and $\operatorname{LTABLE}(j), j=1,2, \ldots, \operatorname{Prod}(\operatorname{IDIM}(i), i=1,2, \ldots$, NDIM, cell frequencies (or probabilities) for the contingency table, and produces as output, all marginal and conditional entropies, transmissions, systematic entropies. SYSENT also produces tables of values to answer a number of 
specific research questions included in this study. Appendix $V$ includes a samples of the output file for SYSENT.

The General Log-Linear/Logit Approach

This section develops the general log-linear and logit expressions and models used to address the research questions, where applicable. As will be demonstrated, certain research questions have been addressed by Green and Carmone or others, in the development of segment congruence analysis while certain other questions have not applied in that context.

of those questions which have not been addressed in segment congruence analysis, certain ones have been addressed in other contexts, using log-linear models, while certain others, though not addressed by any major published material, could conceivably be addressed by these models. Yet a fourth category of research questions, or certain aspects within them, cannot be addressed by the general loglinear models. The reader unfamiliar with the general loglinear and logit models should refer to Appendix I for a brief introduction to these models.

This section employs the same variables which were used to discuss the information theoretic approach. I.e., the segmentation variables are assumed to be $A, B, C$, and $D$; 
while the descriptor variables are assumed to be $X, Y$, and Z.

Phase I: Analysis of The Segmentation Variables. As noted in Chapter 2, one major shortcoming of the general log-linear models is their inability to generate a measure of the amount of association. In addressing the first three. questions, the information theoretic approach generated methods of measuring the amount of association as well as a measure of the test of independence and/or significance. In applying the general log-linear models, however, only a measure of significance (of approximation to the system by the model) is generated. (One should note, however, that significance tests for both information theoretic and log linear methods are actually done using the likelihood-ratio chi-square).

Research Question \#1. The general log-linear model for independence (i.e., the main-effects model is used to address research question \#l (i.e., Are the segmentation variables mutually associated? If yes, how can this mutual association be measured?). This model for a four-variable system $A B C D$ has the following form:

$$
\text { (18) } \log P_{i j k l}=u+u_{i}^{A}+u_{j}^{B}+u_{k}{ }^{C}+u_{l} D
$$

If the null hypothesis of independence is rejected for the above model, it is concluded that statistically 
significant overlap exists among the variables in the multivariate system. The likelihood-ratio chi-square is used to test the null hypothesis.

No measure of the amount of overlap, which is an important issue in segment congruence analysis, is provided by this approach.

Research question \#2. The second research question (i.e., Which basis (or set of bases) makes the highest contribution toward the mutual association?) can be addressed using the log-linear model, for all two-way interaction effects. Both Green and Carmone (1977) and Van Auken and Lonial (1984) have misinterpreted this question. They assessed the contribution of each segmentation variable to the joint variability (systematic entropy) in the system, but used it for assessing mutual association (transmission). It is given (Broekstra 1982) that:

(19) $T_{A}(B: C: D)=T(A: B: C: D)-T(A: B)-T(A: C)-T(A: D)$

$T(A: B: C: D)-T_{A}(B: C: D)$ assesses the contribution of $A$ to the mutual association (i.e., the effect of $A$ being constant, or given, and this, as just shown, is equal to the sum of the bivariate transmissions including $A$ ). The single-variable independence model in the log-linear approach, which is presented later in this chapter, examines 
contributions not to mutual association but to the joint uncertainty as follows:

$$
\begin{aligned}
& T(A: B: C: D)-T(B: C: D) \text {, or equivalently; } \\
& S(A: B: C: D)-S_{A}(B: C: D) \text {. }
\end{aligned}
$$

This was established by the fact that the same likelihood-ratio chi-square obtained for the ajove information theoretic model and the single-variable independent model, using both Green and Carmone data and the data analyzed in this study.

To assess the contribution of each variable (or set of variables) to the total mutual association, models similar to the following must be employed:

$$
\begin{aligned}
& \text { II: (A:B)(A:C)(A:D) } \Rightarrow \log P_{1 j k l}=u+u_{1} A+u_{j}^{B}+u_{k}^{C} \\
& +u_{1}^{D}+u_{1 j}{ }^{A B}+u_{i k^{A C}}+u_{i 1}^{A D} \\
& (B: A)(B: C)(B: D) \Rightarrow \log P_{i j k 1}=u+u_{i}^{A}+u_{j}^{B}+u_{k}^{C} \\
& +u_{1}^{D}+u_{1 j}^{A B}+u_{y k} B C+u_{y 1}{ }^{B D} \\
& \text { (C:A)(C:B)(C:D) } \Rightarrow>\log P_{\{j k\}}=u+u_{1}^{A}+u_{y}^{B}+u_{x} c \\
& +u_{1}^{D}+u_{1 k}^{A C}+u_{j k}{ }^{B C}+u_{k I}^{C D} \\
& (D: A)(D: B)(D: C) \rightarrow \log P_{1 j k I}=u+u_{1}^{A}+u_{j}^{B}+u_{k}^{C} \\
& +u_{I} D+u_{11} \lambda D+u_{j I B D}+u_{k I} C D
\end{aligned}
$$

This model can then be compared with the independence model with respect to the drop in its likelihood ratio chisquare. The null hypotheses are stated as; e.g., the model $\{A B\}\{A C\}\{A D\}$ fits the data. If this were true, a small 
likelihood ratio chi-square would be obtained, which, in turn, would result in tentatively accepting the hypothesis that $\mathrm{A}$ is significantly associated with $\mathrm{B}, \mathrm{C}$, and $\mathrm{D}$.

In order to understand the implications of the above model, one can use its equivalent test in information theory. As mentioned, $\{A B\}\{A C\}\{A D\}$ is equivalent to $T_{A}(B C D)$. Testing the above log-linear model is, therefore, equivalent to testing whether, given $A ; B, C$, and D are independent (i.e., $\left.H_{0}: T_{A}(B C D)=0\right)$. Accepting such hypothesis (which will result from small likelihood-ratio chi-square) would mean that the significant association observed in the first research question is due to $A$ and not B, C, or D. Rejection of such hypothesis would indicate that, at least, by holding A constant, there still exists association among the remaining variables. Here, then, one would search for the variable which removes a greater amount of association than the rest.

For this model, also, the log-linear approach does not provide a measure of strength of the relation. The loglinear analysis only indicates whether a model fits statistically, but not how much overlap exists.

Research Question \#3. In order to address the third research question (i.e.,_which basis, or set of bases, makes the highest contribution toward the joint variability in the system of segmentation bases?), the single-variable (or single-clustering) log-linear model is utilized: 


$$
\begin{aligned}
& (A)(B C D) \Rightarrow \log P_{1 j k 1}=u+u_{1}^{A}+u_{j}^{B}+v_{k}^{C}+u_{1}^{D} \\
& +u_{j x}{ }^{B C}+u_{j 1} B D+u_{k 1}{ }^{C D}+u_{j k 1}{ }^{B C D} \\
& \text { (B) (ACD) } \rightarrow \log P_{1\} k 1}=u+u_{1}^{A}+u_{y}^{B}+u_{k}^{C}+u_{1}^{D} \\
& +u_{1 k} A C+u_{11}^{A D}+u_{x_{1}} C D_{+} u_{1 \times 1} A C D \\
& \text { (C) (ABD) } \Rightarrow \log P_{1 j k 1}=u+u_{1}^{A}+u_{y}^{B}+u_{k}^{C}+v_{1}^{D} \\
& +u_{11}{ }^{A B}+u_{11}{ }^{A D}+u_{11} B D_{+}+u_{i j 1}{ }^{A B D} \\
& \text { (D) (ABC) } \Rightarrow \log P_{1 j k 1}=u+u_{1}^{A}+u_{j}^{B}+u_{k}^{C}+u_{1}^{D} \\
& +u_{1 j}{ }^{A B}+u_{1 k}{ }^{A C}+u_{j k}{ }^{B C}+u_{1 j k}{ }^{A B C} \\
& \text { (AB) (CD) } \Rightarrow \log P_{1 j k 1}=u+u_{1} A+u_{y}^{B}+u_{k}^{C}+u_{1}^{D} \\
& +u_{11}{ }^{A B}+u_{x 1}^{C D} \\
& \text { (AC) (BD) } \Rightarrow>\log P_{1\} k 1}=u+u_{i}{ }^{A}+u_{j}^{B}+u_{k} c+u_{1}^{D} \\
& +u_{12}{ }^{A C}+u_{y_{2}}^{B D}
\end{aligned}
$$

This model hypothesizes that the model for the independence of the segmentation variable (or set) under the consideration is a good model of the data. Likelihood-ratio chi-square is used here to assess this hypothesis for each variable of group of variables. Small values for the likelihood ratio chi-squares would indicate that its corresponding model (e.g., $\{A\}\{B C D\})$ fits the data adequately, and, therefore the variable under consideration is independent of the rest (e.g., A is independent of BCD). Again, the log-linear approach, unlike the information theoretic approach, does not generate a direct measure of association among the variables in any sense. It should be mentioned, however, that the likelihood-ratio chi-squares generated by the models for research questions one thru 
three have been used as measures of association as well. Both Green and Carmone (1977) and Van Auken and Lonial (1984) used the likelihood-ratio chi-square in this way. Research question \#4. Research question \#4 (i.e., Knowledge of which base (or set of bases) makes the highest contribution toward the reduction of uncertainty in the overall system of segmentation bases?) has not been addressed in the context of log-linear models. This is due to the fact that the notion of total uncertainty in a system has not been investigated in this area. However, it is theoretically possible to address this issue by examining the likelihood-ratio chi-square for the no-effects model. The measure obtained is a linear function of the information theoretic measure of uncertainty (i.e., entropy):

The no-effects is stated as:

$$
\log \hat{F}_{i j}=u
$$

where

$$
\text { then } \begin{aligned}
& u=(I / I J K \ldots) \Sigma_{i} \Sigma_{j} \Sigma_{k} \ldots \log F_{i j k} \ldots \\
& L_{L L}^{2}=-2 N \log (2)(H)+2 N \log (A) \\
&=-2 N \log (2)\left[H-H_{M A X}\right]
\end{aligned}
$$

Obviously, calculating $\mathrm{H}$ alone is much simpler (and more logical) than using the log-linear no-effect model and then testing it for significance using the likelihood ratio chisquare. This becomes particularly evident if it is 
necessary to assess the difference between two uncertainties (as is the case here).

In order to assess the contribution of a variable to the total uncertainty in the system, using the general loglinear model, the no-effects model for both the total system and the variable under consideration must be examined.

Then, the uncertainty should be computed by linear transformations of the likelihood-ratio chi-square for both methods (i.e., such that the H terms are calculated). Then, the difference between the two (i.e., the overall system and the variable under the consideration) should be computed.

Furthermore; most standard computer packages (e.g., SAS or SPSS ${ }^{X}$ ) do not even allow the consideration of the noeffects model. It would be very cumbersome to perform the necessary calculations manually particularly for large tables. This is due to the requirement by the log-linear models to generate the expected cell frequencies by the maximum likelihood estimation method first and, then, calculate the likelihood-ratio chi-square.

Research Question \#5. The fifth research question (i.e., Which variable (or set of variables) has the greatest number of significant interrelationships with other variables?) is addressed by examining the log-linear independence models two-variable combinations of the system of segmentation variables. 
(24)

$$
\begin{aligned}
\{A\}\{B\} \Rightarrow & \log P_{j j k l}=u+u_{i}{ }^{A}+u_{j}{ }^{B} \\
& \{A\}\{C\}=u_{i}{ }^{A}+u_{k} C \\
& \{A\}\{D\} \Rightarrow \log P_{i j k l}=u+P_{i j k l}=u+u_{i}^{A}+u_{I} \\
& \{B\}\{A\} \Rightarrow \log P_{i j k l}=u+u_{j} B+u_{i}{ }^{A} \\
& \{B\}\{C\} \Rightarrow \log P_{i j k l}=u+u_{j B}+u_{k D} \\
& \{B\}\{D\} \Rightarrow \log P_{i j k I}=u+u_{j}+u_{I} \\
& \text { etc. }
\end{aligned}
$$

The variable for which more of these bivariate models are significant (i.e., greatest number of bivariate combinations for it depart from independence significantly) is regarded as the variable with the greatest number of dyadic relations. This variable, therefore, plays a more central role as it has interactions with more components in the system.

Here, also, the information theoretic approach provides a direct measure of association, while, the loglinear approach does not.

Phase II: Selection and Analysis of the Distinguished Segmentation Variable. Using the log-linear/logit approach the four research questions in this phase are approached as follows:

Research Question \#1. Which one of the candidate distinguished segmentation bases, identified in phase $I$, is best predicted once we have the knowledge of a set of relevant independent variables (i.e., which set comprises the best distinguished segmentation base)? Multinomial 
logit models are suitable in addressing this question. The simple (i.e., the two-by-two model) is presented below:

$$
\begin{aligned}
& \text { (A) } B Y(X)(Y)(z) \Rightarrow a_{j k 1^{\lambda}}=b^{\lambda}+b_{j} \lambda X \\
& +b_{x} X_{+} b_{1} \lambda z+ \\
& \text { (B) BY }(X)(Y)(z) \Rightarrow O_{j k 1}{ }^{B}=b^{B}+b_{j}^{B X} \\
& +b_{x}^{B Y}+b_{1}^{B 2}+\cdot \\
& \text { (c) } B Y(X)(y)(z) \rightarrow o_{1 f k l^{c}}{ }^{c} b^{c}+b_{j} c X \\
& +b_{k} C Y+b_{1} c z+ \\
& \text { (D) } B X(X)(Y)(2) \Rightarrow O_{1 j k 1} D=b^{D}+b_{f} D X \\
& +b_{x}^{D Y}+b_{1} D Z+
\end{aligned}
$$

All classes of each variable in the contingency table can be estimated as a function of the other variables in the model. The likelihood ratio chi-square is used to evaluate the model. The smaller the likelihood-ratio chi-square, the better the model fits the data.

The logit models shown above are known as the maineffects models. In general, if these models do not produce good estimations of cell frequencies statistically, highereffects models will be introduced.

Research Question \#2. Similar to the information theoretic counterpart of this research question, the logit models developed in the first research question can be utilized to address this research question. Here, the dependent variables are dichotomous, each one having a group 
of particular interest as one class and the other groups as the second class.

Research Question \#3. Green and Carmone (1977)

briefly mentioned that the magnitude of the coefficients of the logit model can be used to address the third research question in phase II. This research question is stated as: How can one prioritize the effect of the independent variables on the distinguished set?

This approach is not practical, because the number of coefficients generated for each independent variable is equivalent to the degrees of freedom for a two way contingency table, with the dependent and the independent variable as its dimensions. In estimating each cell frequency from the parameters produced by the logit model, one need to decide which coefficients are present and then sum these coefficients. Then the antilogarithm of this sum gives the estimated odds of a particular class of the dependent variable against all other classes.

In the applications used by Green and Carmone (1977) and Van Auken and Lonial (1984), all of the variables were dichotomized. In such case, only one coefficient is generated for each two dimensional contingency table of the dependent variable and a dependent variable. Here, the coefficients can be used to judge the significance of each variable. However, there is no collective way to judge the significance of a variable based on its coefficients, as 
suggested by Green and Carmone (1977), if any of the variables used is multichotomous.

The general log-linear model can be used to develop successive models (much the same as the information theoretic approach for this question) of significance of variables in a marginal and partial sense. First, a set of two-variable independence models (of the distinguished variable and the descriptor variables) are run and the most significant variable is identified $(e . g .,\{A\}\{B\},\{A\}\{C\}$, etc.). Then, a set of partial single-variable independence models are run composed of the distinguished variable versus the most significant variable found above and the remaining variables (e.g., assuming that $B$ was selected as the most significant variable, $\{A\}\{C B\},\{A\}\{C D\}$, etc.). Repeating this process to include all combinations (with the last one being $(A\}\{B C D E . .\}$.$) will result in a ranking identical to$ that of the information theoretic approach.

Research question \#4. The fourth research question (i.e., What is the dependency structure in each class of the distinguished segmentation base? How can it be quantified?) has neither been addressed directly, nor indirectly, in the segment congruence analysis context. Nevertheless, the HILOGLINEAR procedure in SPSSX, generated parameter estimates, which in turn can be used to develop a diagram of effects, including magnitudes of effects. This is the essence of dependency analysis. 
Tables VII and VIII present the algebraic expressions, if applicable, for the research questions in the two phases, respectively.

Summary of the Methodology

Properties and theoretical underpinnings of information theory were detailed in the last section of Chapter 2 and in Appendix II. It was established that the information theoretic approach has numerous properties that make it a viable technique for data analysis. For instance, the decomposability property of informational measures and the analogy between these measures and variance (Garner and McGill, 1956) enables one to perform ANOVA like analyses of data with discrete dependent variables. Furthermore, this property provides a powerful structural modeling tool for researchers concerned with the analysis of nominally scaled data, in all fields (Broekstra, 1978, 1981, 1982, Krippendorff, 1979, 1981, KIir, 1976, 1986, etc.).

The methodology section of the present chapter established the fact that the information theoretic approach is capable of addressing all of the question concerning segment congruence analysis raised by this undertaking. Table IX presents a list of these research questions and the capability of the information theoretic approach as well as the log-linear approach to segment congruence analysis. 
As Table IX suggests the information theory is capable of addressing all issues discussed within the framework of segment congruence analysis. 
TABLE VII

\section{INFORMATION THEORETIC AND LOG-IINEAR MATHE- MATICAL EXPRESSIONS USED FOR SEGMENT CONGRUENCE ANALYSIS (PHASE I)}

\section{QI.I) Overlap}

IT: $\quad T(A: B: C: D)=H(A)+H(B)+H(C)+H(D)-H(A, B, C, D)$ where;

$H(A, B, C, D)=-\Sigma_{i} \Sigma_{j} \Sigma_{k} \Sigma 1 \quad P\left(A_{i}, B_{j}, C_{k}, D_{1}\right) \log _{2} P\left(A_{i}, B_{j}, C_{k}, D_{1}\right)$

$L L: \quad\{A\}\{B\}\{C\}\{D\} \Rightarrow \log P_{i j k l}=u+u_{i}^{A}+u_{j}{ }^{B}+u_{k} C+u_{l} D$

\section{Q I.2) Contribution to Overlap}

IT:

etc.

$$
\begin{aligned}
& \Phi_{1} T=T(A: B: C: D)-T_{A}(B: C: D) \\
& \Phi_{2} T=T(A: B: C: D)-T_{B}(A: C: D) \\
& \Phi_{3} T=T(A: B: C: D)-T_{C}(A: B: D) \\
& \Phi_{4} T=T(A: B: C: D)-T_{D}(A: B: C) \\
& \Phi_{5} T=T(A: B: C: D)-T_{A B}(C: D) \\
& \Phi_{6} T=T(A: B: C: D)-T_{A C}(B: D)
\end{aligned}
$$

II: $\{A: B\}\{A: C\}\{A: D\} \Rightarrow \begin{aligned} & \log P_{i j k I}=u+u_{i} A+u_{j} B+u_{k} C \\ &+u_{I}+u_{j j} A B+u_{j k} C^{C}+u_{i l} A D\end{aligned}$

$$
\begin{aligned}
& \{B: A\}\{B: C\}\{B: D\} \Rightarrow \log P_{i j k l}=u+u_{i}{ }^{A}+u_{j} B+u_{k} C \\
& +u_{1}{ }^{D}+u_{i j} A B+u_{j k} B C+u_{j l} B D
\end{aligned}
$$

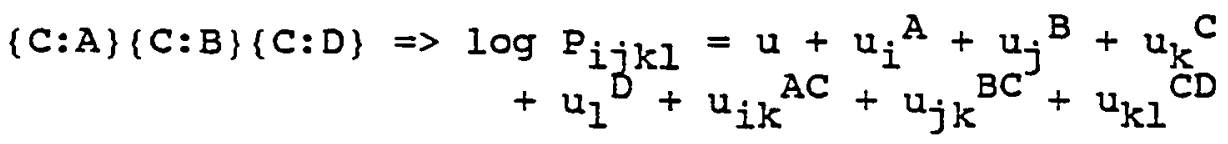

$\{D: A\}\{D: B\}\{D: C\} \Rightarrow \log P_{i j k l}=u+u_{i}^{A}+u_{j} B+u_{k} C$

$$
+u_{1}+u_{i I} A D+u_{j I B D}+u_{k I} C D
$$


Table VII - (continued)

\section{Q I.3) Contribution to Joint Variability}

IT:

$$
\begin{aligned}
\Phi_{1} S & =S(A: B: C: D)-S_{A}(B: C: D) \\
\Phi_{2} S & =S(A: B: C: D)-S_{B}(A: C: D) \\
\Phi_{3} S & =S(A: B: C: D)-S_{C}(A: B: D) \\
\Phi_{4} S & =S(A: B: C: D)-S_{D}(A: B: C) \\
\Phi_{5} S & =S(A: B: C: D)-S_{A B}(B: C) \\
\Phi_{6} S & =S(A: B: C: D)-S_{A C}(B: D) \\
\text { etc. } &
\end{aligned}
$$

LL: $\begin{aligned}\{A\}\{B C D\} \Rightarrow>\log P_{i j k l} & =u+u_{i}{ }^{A}+u_{j} B+u_{k} C+u_{l} D \\ & +u_{j k} B C^{B}+u_{j l} B D+u_{k l} C D+u_{j k l} B C D\end{aligned}$

$\begin{aligned}\{B\}\{A C D\} \Rightarrow \log P_{i j k l} & =u+u_{i}^{A}+u_{j}^{B}+u_{k} C+u_{I} D \\ & +u_{i k} A C^{A}+u_{i l} A D+u_{k l} C D_{1}+u_{i k l} A C D\end{aligned}$

$\{C\}\{A B D\} \Rightarrow \log P_{i j k I}=u+u_{i}^{A}+u_{j}^{B}+u_{k} C+u_{I} D$ $+u_{i j} A B+u_{i l} A D+u_{j l} B D_{1} u_{i j l} A B D$

(D) $\{A B C\} \Rightarrow \log P_{i j k I}=u+u_{i}^{A}+u_{j}^{B}+u_{k} C+u_{I} D$ $+u_{i j} A B+u_{i k} A C+u_{j k} B C+u_{i j k} A B C$

$\begin{aligned}\{A B\}\{C D\} \Rightarrow \log P_{i j k l} & =u+u_{i}^{A}+u_{j}^{B}+u_{k} C+u_{l}{ }^{D} \\ & +u_{i j}{ }^{A}+u_{k l}\end{aligned}$

$\begin{aligned}\{A C\}\{B D\} \Rightarrow \log P_{i j k l} & =u+u_{i}^{A}+u_{j}^{B}+u_{k} C+u_{l}^{D} \\ & +u_{i k} A C^{2}+u_{j l} B\end{aligned}$

etc. 
Table VII - (continued)

\section{Q I.4) Reduction of Uncertainty in the System}

IT:

$$
\begin{aligned}
& \Phi_{1} H=H(A, B, C, D)-H(A) \\
& \Phi 2 H=H(A, B, C, D)-H(B) \\
& \Phi 3 H=H(A, B, C, D)-H(C) \\
& \Phi 4 H=H(A, B, C, D)-H(D) \\
& \Phi_{5} H=H(A, B, C, D)-H(A B) \\
& \text { क6 } H=H(A, B, C, D)-H(A C) \\
& \text { etc. }
\end{aligned}
$$

IL: $\{\cdot\}\{\cdot\} \Rightarrow \log P_{i j k l}=u-\log P_{i}=u_{i}$

$$
\begin{aligned}
& \{\cdot\}\{\cdot\} \Rightarrow \log P_{i j k l}=u-\log P_{j}=u_{j} \\
& \{\cdot\}\{\cdot\} \Rightarrow \log P_{i j k l}=u-\log P_{k}=u_{k} \\
& \{\cdot\}\{\cdot\} \Rightarrow \log P_{i j k I}=u-\log P_{i}=u_{1} \\
& \{\cdot\}\{\cdot\} \Rightarrow \log P_{i j k I}=u-\log P_{i}=u_{i j} \\
& \{\cdot\}\{\cdot\} \Rightarrow \log P_{i j k I}=u-\log P_{i}=u_{i k} \\
& \text { etc. }
\end{aligned}
$$

\section{Q I.5) Number of Dyadic Relations}

IT:

$$
\begin{aligned}
& T(A: B), T(A: C), T(A: D) \\
& T(B: A), T(B: C), T(B: D) \\
& T(C: A), T(C: B), T(C, D) \\
& \text { etc. }
\end{aligned}
$$

LI: $\quad\{A\}\{B\} \Rightarrow \log P_{i j k l}=u+u_{i A}^{A}+u_{j} B$

$\{A\}\{C\} \Rightarrow \log P_{i j k l}=u+u_{i A}+u_{k} C$
$\{A\}\{D\} \Rightarrow \log P_{i j k l}=u+u_{i}+u_{I}$

$\{B\}\{A\} \Rightarrow \log P_{i j k l}=u+u_{j B}^{B}+u_{i C}^{A}$

$\{B\}\{C\} \Rightarrow \log P_{i j k l}=u+u_{j B}+u_{k D}$

$\{B\}\{D\} \Rightarrow \log P_{i j k I}^{i j k I}=u+u_{j} B+u_{I}$

etc. 
$109(a)$

TABLE VIII

INFORMATION THEORETIC AND LOG-IINEAR MATHE-

MATICAL EXPRESSIONS USED FOR SEGMENT

CONGRUENCE ANALYSIS (PHASE II)

QII.I) Predictability

IT:

$$
\begin{aligned}
& \Phi H(A)=H(A)-H_{X Y Z}(A) \\
& \Phi \mathrm{H}(\mathrm{B})=\mathrm{H}(\mathrm{B})-\mathrm{H}_{X Y Z}(\mathrm{~B}) \\
& \Phi H(C)=H(C)-H_{X Y Z}(C) \\
& \Phi \mathrm{H}(\mathrm{D})=\mathrm{H}(\mathrm{D})-\mathrm{H}_{\mathrm{XYZ}}(\mathrm{D}) \\
& \Phi H(A B)=H(A B)-H_{X Y Z}(A B) \\
& \text { etc. } \\
& \Phi \cdot H(A C)=H(A C)-H_{X Y Z}(A C)
\end{aligned}
$$

IL: $\begin{aligned}(A) B Y\{X\}\{Y\}\{Z\} \Rightarrow o_{i j k I}{ }^{A}=b^{A} & +b_{j}^{A X}+b_{k}^{A Y} \\ & +b_{1}^{A Z}+e\end{aligned}$

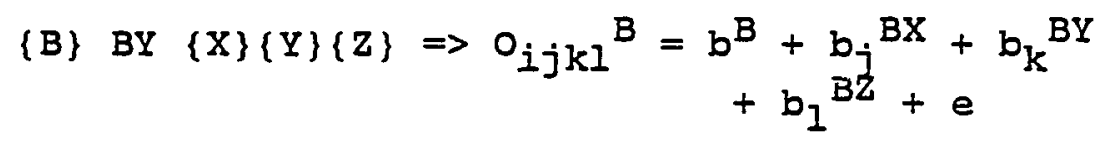

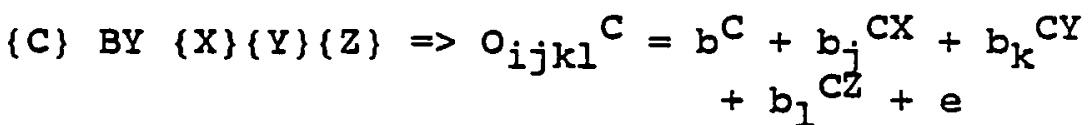

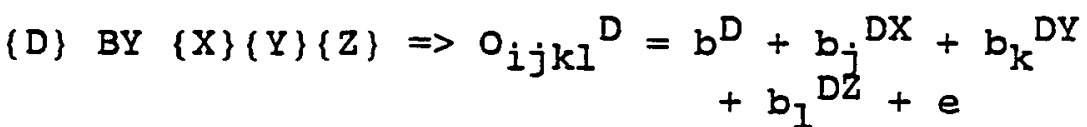

where; e.g.,

$O_{i j k l}^{A}=\log \left(p_{(j j k l}{ }^{A=1} / 1-p_{i j k l}{ }^{A=1}\right)$,

$b^{A}, b_{j} A X, b_{k} A \bar{Y}$, and $b_{1} A Z$ are the parameters

estimated, and

$e$ is the residual

Note: The above logit models are known as the main-effects models. In general, if these models do not produce good estimations of cell frequencies statistically, highereffects models will be introduced. 
Table VIII - (Continued)

Q II.2) Information/Noise ratio (Systematic entropy)

Same as $Q$ II.I after reclassifications of the distinguished variables.

QII.3) Prioritization

$I T: H(A)=T(A: X)+T_{X}(A: Y)+T_{X Y}(A: Z)+H_{X Y Z}(A)$

LL: Magnitudes of coefficients in the logit model

QII.4) Dependency Analysis

IT: Conant's (1982) Dependency Analysis

IL: The prioritization scheme developed in Q II.3 
TABIE IX

COMPARISON OF THE TWO APPROACHES TO SEGMENT CONGRUENCE ANAIYSIS

\begin{tabular}{|c|c|c|c|c|}
\hline \multicolumn{2}{|c|}{ QUESTION } & \multirow[b]{2}{*}{ THEORY } & \multirow[b]{2}{*}{ LOG-IINEAR } & \multirow{2}{*}{$\begin{array}{l}\text { APEIIED } \\
\text { TO SCA } \\
\text { BEFORE }\end{array}$} \\
\hline NUMBER & DESCRIPTION & & & \\
\hline QI.I & $\begin{array}{l}\text { OVERLAP: } \\
\text { (EXISTENCE) } \\
\text { (AMOUNT) }\end{array}$ & $\star$ & + & $\begin{array}{l}\text { YES } \\
\text { NO }\end{array}$ \\
\hline ㅇ.2 & $\begin{array}{l}\text { CONTRIBUTION } \\
\text { TO OVERIAP: } \\
\text { (EXISTENCE) } \\
\text { (AMOUNT) }\end{array}$ & * & $\ddot{\#}$ & $\begin{array}{l}\text { NO } \\
\text { NO }\end{array}$ \\
\hline 요.3 & $\begin{array}{l}\text { CONTRIBUTION } \\
\text { TO JOINT VAR: } \\
\text { (EXISTENCE) } \\
\text { (AMOUNT) }\end{array}$ & $\begin{array}{l}\star \\
\star\end{array}$ & +1 & $\begin{array}{l}\text { YES } \\
\text { NO }\end{array}$ \\
\hline Q I. I & $\begin{array}{l}\text { CONTRIBUTION TO } \\
\text { UNCERTAINTY: } \\
\text { (EXISTENCE) } \\
\text { (AMOUNT) }\end{array}$ & $\star$ & $\hat{\wedge} 2$ & $\begin{array}{l}\text { No } \\
\text { No }\end{array}$ \\
\hline Q I.5 & $\begin{array}{l}\text { NUMBER OF DYADIC } \\
\text { RELATIONS }\end{array}$ & * & $\#$ & No \\
\hline 2 II.I & PREDICTABILITY & * & + & YES \\
\hline ㅇII.2 & $\begin{array}{l}\text { REFINEMENT OF } \\
\text { PREDICTABILITY }\end{array}$ & * & $\#$ & No \\
\hline ㅇII.3 & PRIORITIZATION & * & $\#^{3}$ & YES \\
\hline QII.4 & $\begin{array}{l}\text { DEPENDENCY } \\
\text { ANALYSIS }\end{array}$ & $\star$ & $\sim$ & No \\
\hline $\begin{array}{l}\bar{\star}=\text { Fir } \\
\#=\text { Fir } \\
t=\text { Reg } \\
-=\text { Has } \\
\hat{A}=\text { Has }\end{array}$ & $\begin{array}{l}\text { done in this thesis } \\
\text { t done in this thesis } \\
\text { rted earlier in litera } \\
\text { not been, but can easi } \\
\text { not been done (too cum }\end{array}$ & $\begin{array}{l}\text { (new pr } \\
\text { (using } \\
\text { ture } \\
\text { ly be d } \\
\text { bersome }\end{array}$ & $\begin{array}{l}\text { rams) } \\
\text { ss') }\end{array}$ & cogram) \\
\hline
\end{tabular}

1 The chi-square statistic has been used to make comparisons between models.

2 Appropriate no-effects models must be developed and their corresponding likelihood-ratio chi-squares must be computed to assess existence and amount.

3 Using coefficients of the logit function has been suggested for this purpose, but it only applies to cases where all variables are dichotomous. 
THE DATA AND PROCEDURES

A survey conducted by the Bonneville Power Administration (BPA) in collaboration with the Pacific Northwest Utilities Conference Committee (PNUCC) and the Northwest Regional Power Planning Council (NRPPC) provides the basis for an empirical application of segment congruence analysis using the information theoretic approach. Among the existing techniques, the general log-linear and logit models are applied to this data as well, in order to demonstrate the efficacy of the information theoretic methods.

The Pacific Northwest Residential Energy Survey (PNWRES), was designed to gain knowledge on issues of energy use habits, attitudes towards energy use and conservation, and the extent of conservation activities (practices and installations) in the Pacific Northwest region. The information is used to support activities which are authorized by the Pacific Northwest Electric Power Planning and Conservation Act of 1980.

\section{Description of the study}

The survey was administered during the summer months of 1983. The population was approximated at three million dwellings in the Pacific Northwest region. of the original sample size of 6227 accounts, 546 were determined to be 
ineligible, and 481 respondents refused to complete the interview. Also, interviewers were unable to contact an eligible respondent in 345 cases.

Overall, approximately nine percent (9\%) of the accounts were determined to be ineligible and approximately eighty six percent $(86 \%)$ of the eligible residents responded to the survey, an overall completion rate of over seventy six percent (76\%). This amounted to a total sample size of 4703. The field work was conducted by Louis B. Harris and Associates, Inc.

According to the PNWRES User's Guide, "data collection began in late May 1983, and was completed in September 1983. Over 80 percent of the interviews were conducted in June and July." (PNWRES User's Guide, 1983) Eligibility of a respondent household consisted of existing service from an electricity utility to the home in question. Emphasis was placed upon residential meters, one residential unit per meter, and year-round as opposed to seasonal occupation of the home.

Average interview time was approximately one hour, and all surveys were edited, keypunched, and 100 percent verified. Ten percent of interviews were verified by phone. First, utilities serving fewer than 1000 customers were grouped together or combined with larger utilities. Second, three of the larger privately owned utilities were disaggregated on the basis of political (state) boundaries 
and geographic zones. These modifications to the clustering of utilities resulted in: Pacific Power and light being subdivided and represented in every geographic and weather zone except Western Washington; the Washington Water Power Company was represented both in Eastern Washington and Idaho; and Idahe Tower Company was represented both in Idaho and Eastern oregon. This resulted in the generation of 116 mutually exclusive and exhaustive utility groupings.

A multistage cluster sampling technique involving two dimensions of stratification (by six levels of geographic regions and two levels of utility ownership, i.e., public and private) was employed for selecting the sample of utilities for 1983 PNWRES.

Within each of the six geographic strata composed of publicly owned utilities, substrata were constructed independently for each stratum, based upon the number of residential accounts served by utilities within that stratum. Individual utility clusters were then selected within substrata with probabilities proportionate to size, with the number of residential accounts as the measure of size.

All privately owned utilities within the sampling frame were selected with certainty into the survey sample. One pair of privately owned utilities was included in the sample as a utility cluster: Pacific Power and Light's Montana operations and Montana Light and Power Company. 
Following selection of the 57 utility clusters, the number of subclusters (Meter Reading Routes or MIR) within a utility on which interviews were to be attempted was determined to ensure that substrata contributed relatively equal numbers of observations within strata and that strata contributed relatively equal numbers of observations to the total survey sample. Specific MRR's were selected via the proportional to size procedures. Finally, fifteen residential accounts were selected systematically by utilities for each MRR assigned.

variables Measured. Nine general topics were included in the questionnaire for field interviews:

1. Basic dwelling unit characteristics.

2. Energy-related attitudes/opinions.

3. Conservation measures utilized.

4. Space heating fuels and equipment.

5. Water heating fuels and equipment.

6. Air conditioning fuels and equipment.

7. Household appliance characteristics.

8. Resident demographics.

9. Physical measurement of dwelling unit and water temperature.

With the consent of the respondent, interviewers measured the temperature of the hot water and the outside dimensions of the dwelling unit living space. Also, respondents were asked to sign a "Waiver Form" permitting 
Bonneville Power Administration to access the respondents' electricity and, where appropriate, natural gas billing histories from relevant suppliers.

The billing history data includes both the amount of fuel used and the total cost of the fuel for each billing period between September 1981 and December 1982. In order to support the evaluation of Bonneville's residential weatherization program, thirteen utility clusters (nine different utilities) were asked to supply additional billing data for the period ending April 1983.

Additionally, data on weather and climatic changes were collected, in terms of daily maximum and minimum and heating-degree-days and cooling-degree-days for the period between september 1, 1981 and March 31, 1983. This data was collected with the help of National oceanographic and Atmospheric Administration (NOAA) .

\section{Selection and Aggregation of Variables}

A flow chart of procedures used for selection and aggregation of research variables is presented in Figure 9.

The variable selection process began by including all the variables which would (even remotely) lend themselves to a marketing analysis study in the PNWRES data. The arrows by the variable names in Appendix IV point to such variables. Six clusters of segmentation variables were identified using common sense and expert opinion. Several 
officials from the Bonneville Power Administration, BPA, and the Pacific Power and Light Company, PP\&I were interviewed and their judgments were taken into account in determination of relevant groups of variables.

Table $x$ includes a listing of the variables used in the clusterings, their description and their corresponding question numbers in the questionnaire (see Appendix III for the questionnaire and Appendix IV for the complete variable listing).

SPSSX's clustering procedures were utilized to combine related variables into single composite variables. Table XI presents the list of these clusters, their description and the variable names, corresponding to the variable list in Appendix IV, for each cluster.

The questions related to four groups of these variables were so closely related or interwoven within each group that it was easily justified to cluster them together and treat them as aggregates of variables. These four groups included the energy related attitude questions, VAR049 thru VAR055, feelings and perceptions questions about the environment, VAR066 thru VAR073, awareness of funding for energy efficiency improvement programs, VAR074, VAR076, and VAR078, and major uses of electricity variables, VAR365, VAR367, VAR369, VAR371, and VAR373. 

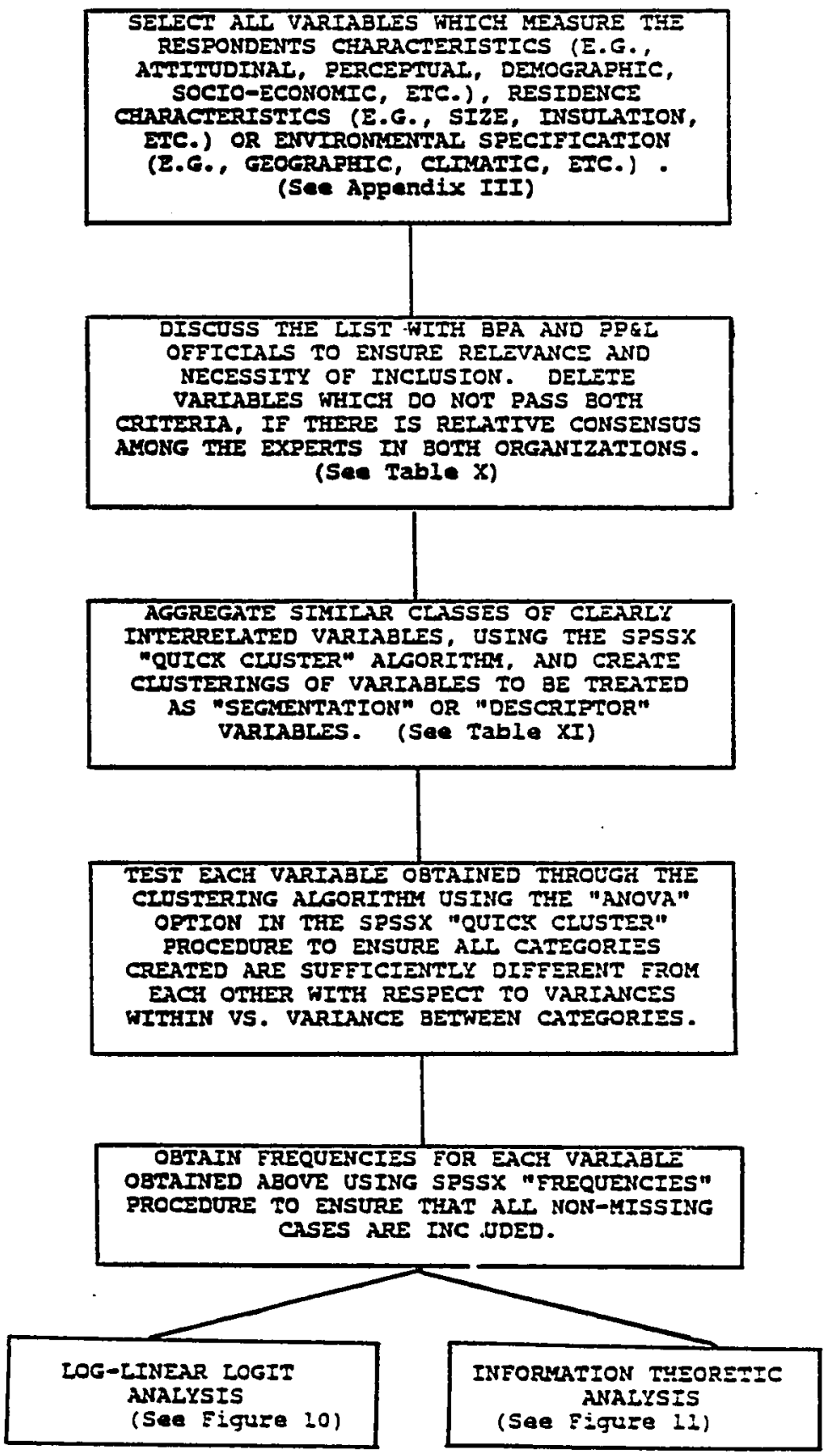

Figure 9. Flow chazt tor selection and aggzegatior: of research variables 


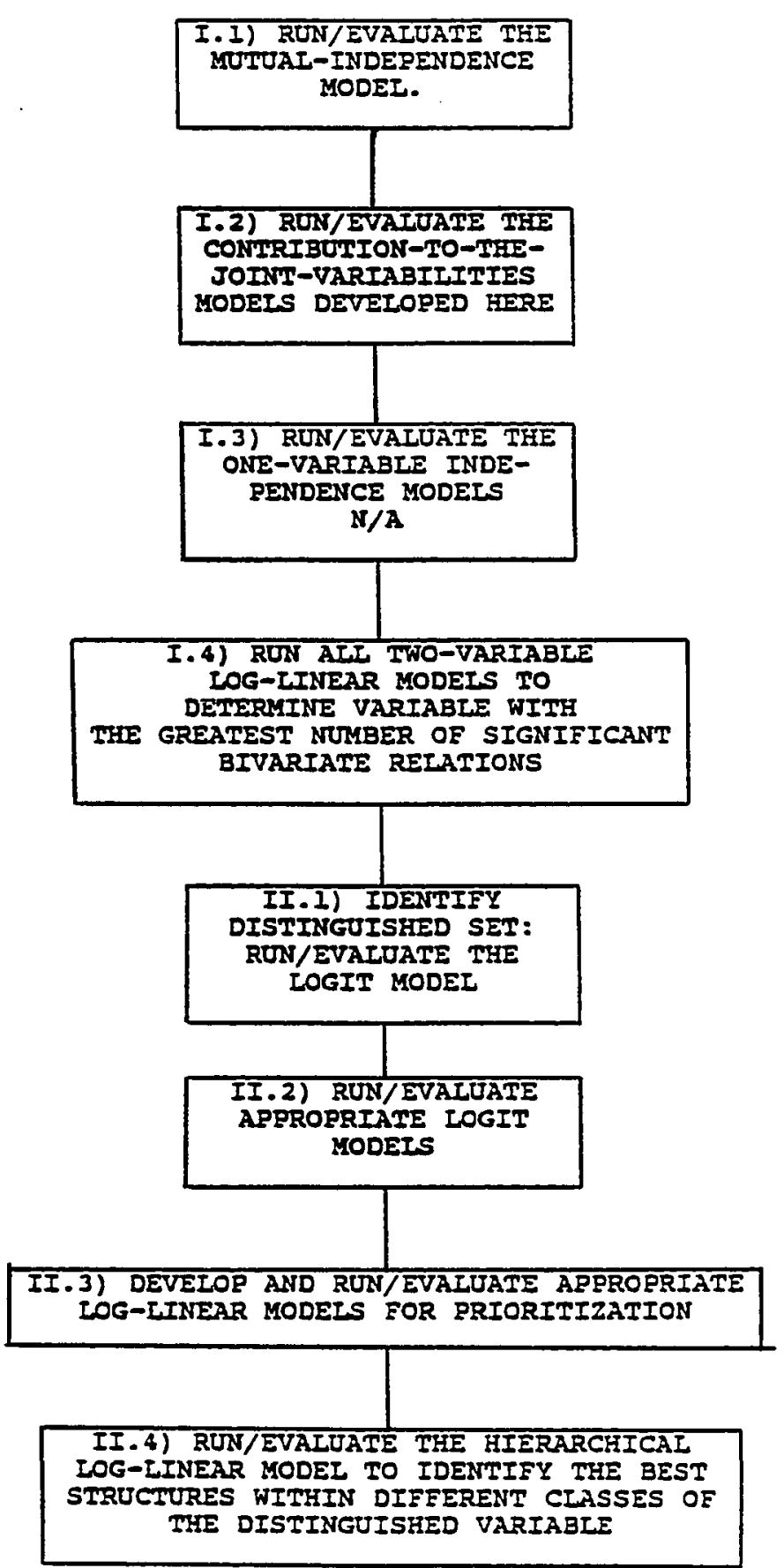

Eigure 10. Flow chart of the log-linear/logit analysis. 


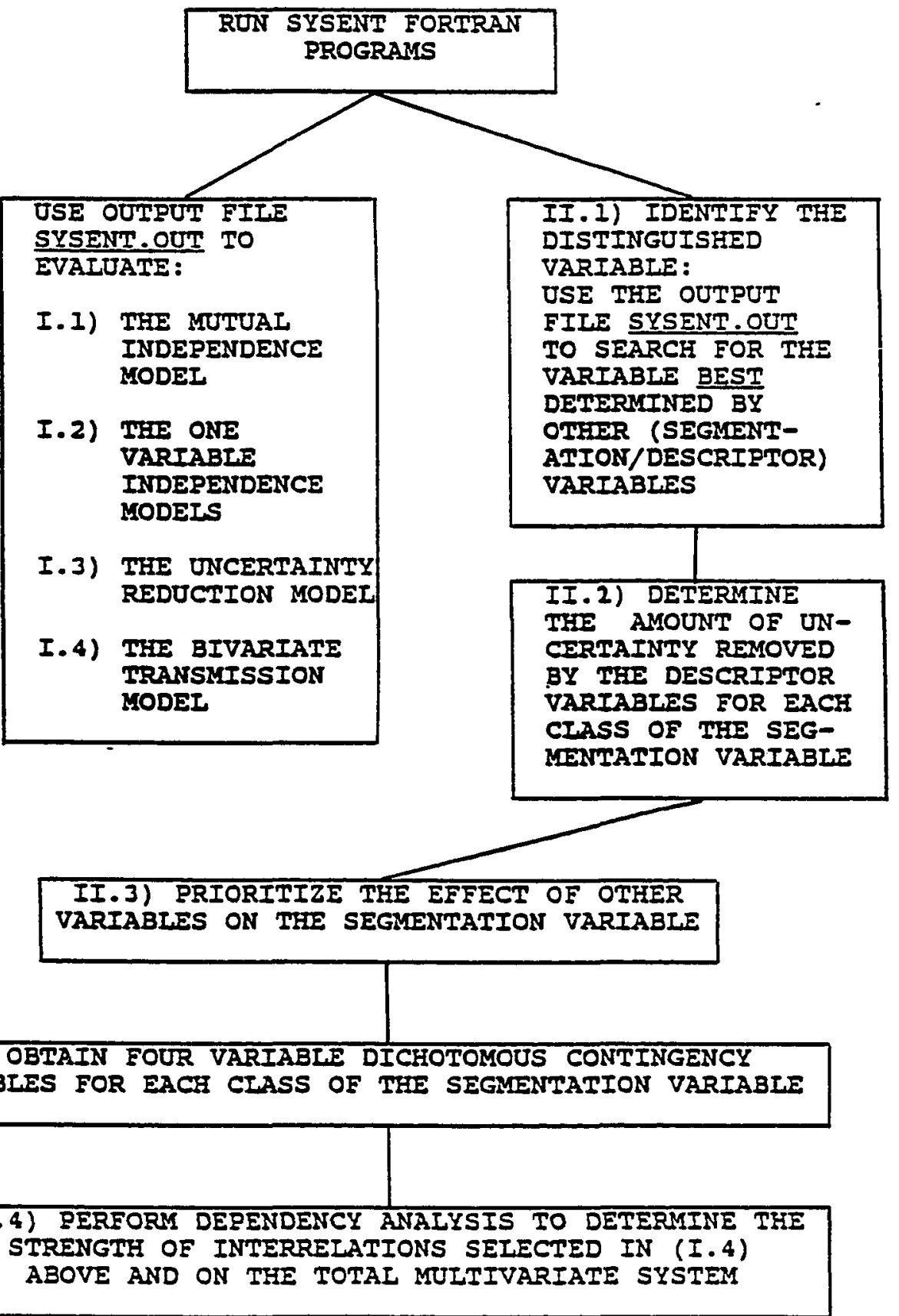

Figure 11. Flow chart of the information theoretic analysis 
TABIE $X$

IIST OF VARIABLES PRIOR TO AGGREGATIONS

\begin{tabular}{|c|c|c|}
\hline $\begin{array}{l}\text { VARIABIE } \\
\text { NAMEE }\end{array}$ & $\begin{array}{l}\text { VARIABLE } \\
\text { LABEL }\end{array}$ & $\begin{array}{l}\text { TION } \\
\text { BER }\end{array}$ \\
\hline $\begin{array}{l}\text { GENATWT } \\
\text { VAR049 } \\
\text { VAR050 } \\
\text { VAR051 } \\
\text { VAR052 } \\
\text { VAR053 } \\
\text { VAR054 } \\
\text { VAR055 }\end{array}$ & $\begin{array}{l}\text { Energy Efficiency of home } \\
\text { Thought on environmental pollution } \\
\text { Thoughts on cost of energy } \\
\text { Thoughts on unemployment } \\
\text { Thoughts on scarcity of energy } \\
\text { Thoughts on inflation } \\
\text { Thoughts on crime }\end{array}$ & $\begin{array}{l}20 \\
20 \\
20 \\
20 \\
20 \\
20 \\
20\end{array}$ \\
\hline $\begin{array}{l}\text { ENRATT } \\
\text { VAR067 } \\
\text { VAR069 } \\
\text { VAR073 }\end{array}$ & $\begin{array}{l}\text { statement-reducing water temp. saves } \\
\text { statement-tuming down temp. worthwhile } \\
\text { statement-conserving energy=change in life }\end{array}$ & $\begin{array}{l}22 \\
22 \\
22\end{array}$ \\
\hline $\begin{array}{l}\text { BERAVE } \\
\text { VAR075 } \\
\text { VAR077 } \\
\text { VAR079 }\end{array}$ & $\begin{array}{l}\text { Made use of federal tax credit } \\
\text { Made use of state tax credit } \\
\text { Made use of low interest loan }\end{array}$ & $\begin{array}{l}24 \\
24 \\
24\end{array}$ \\
\hline $\begin{array}{l}\text { PERCEPT } \\
\text { VARO66 } \\
\text { VAR071 } \\
\text { VAR072 }\end{array}$ & $\begin{array}{l}\text { Statement-comfortable at or }<68 \mathrm{~F} \\
\text { statement-right to use energy } \\
\text { Statement-price more important than energy }\end{array}$ & $\begin{array}{l}22 \\
22 \\
22\end{array}$ \\
\hline $\begin{array}{l}\text { AWARE } \\
\text { VAR074 } \\
\text { VAR076 } \\
\text { VAR078 }\end{array}$ & $\begin{array}{l}\text { Heard of Federal tax credit } \\
\text { Heard of state tax benefit } \\
\text { Heard of low interest loan }\end{array}$ & $\begin{array}{l}22 \\
22 \\
22\end{array}$ \\
\hline$\frac{\text { KWTUSE }}{\text { KWE }}$ & Total kilo-watt-hours used in 12 months & $\star$ \\
\hline$\frac{\text { CLIMGEO }}{\text { GEOAREA }}$ & Geographic area & $\star \star$ \\
\hline $\begin{array}{l}\text { TYPDWEL } \\
\text { VAROOS } \\
\text { VAR469 } \\
\text { VAR470 } \\
\text { VAR471 }\end{array}$ & $\begin{array}{l}\text { Type of dwelling } \\
\text { Total square feet first floor } \\
\text { Total square feet second floor } \\
\text { Total square feet third floor }\end{array}$ & $\begin{array}{l}2 \\
\star \star \\
\star \star \\
\star \star\end{array}$ \\
\hline$\frac{\text { RENTOWN }}{\text { VAROOB }}$ & Means of payment for housing & 5 \\
\hline $\begin{array}{l}\text { DEMOG } \\
\text { VAR318 } \\
\text { VAR360 } \\
\text { VAR362 } \\
\text { VAR364 }\end{array}$ & $\begin{array}{l}\text { Total number of residents } \\
\text { Ethnic origin } \\
\text { Level of education } \\
\text { Combined } 1982 \text { income }\end{array}$ & $\begin{array}{l}129 \\
132 \\
133 \\
134\end{array}$ \\
\hline $\begin{array}{l}\text { INSUL } \\
\text { VARI33 } \\
\text { VARI4I } \\
\star \text { Merged } \\
\star \star \text { No ques }\end{array}$ & $\begin{array}{l}\text { Percent insulation, roof } \\
\text { Percent insulation, floor } \\
\text { from a different questionnaize } \\
\text { stion number listed in the questionnaire. }\end{array}$ & $\begin{array}{l}34 \\
41\end{array}$ \\
\hline
\end{tabular}


The SPSSX QUICK CLUSTER procedure was utilized to aggregate each of the above groups of variables. These clusters were named GENATT, ..., ENRATT, attitudinal cluster, TIRCEPT, perceptual cluster, AWARE, awareness cluster, and BEHAVE, electricity usage behavior. The study began by identifying a set of segmentation variables in two separate stages. In the first stage the a priori method was used to identify a large number of variables. Next, the clustering approach was used to group these variables into reasonable clusters identify the segmentation and descriptor variables. Then again, Phase I and II of research questions were addressed. The results of this study establish a set of market segments useful to energy planners and other decision makers in the field of utilities and power administration.

Chapter IV presents analyses and results of the PNWRES study through the two main approaches (i.e., information theoretic and $\log -1$ inear methods). 
TABLE XI

IIST OF VARIABIES (OR CLUSTERS) AFTER AGGREGATION

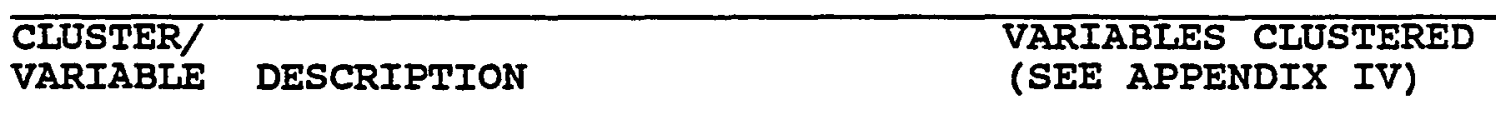

\begin{tabular}{|c|c|c|}
\hline GENATT & $\begin{array}{l}\text { General attitudes } \\
\text { (economy and environment) }\end{array}$ & VAR049 THRU VAR055 \\
\hline ENRATT & Energy related attitudes & $\begin{array}{l}\text { VAR067, VAR069, } \\
\text { VAR073 }\end{array}$ \\
\hline PERCEPT & $\begin{array}{l}\text { Energy (comfort/discomfort) } \\
\text { perceptions }\end{array}$ & $\begin{array}{l}\text { VAR066, VAR071, } \\
\text { VAR072 }\end{array}$ \\
\hline AWARE & $\begin{array}{l}\text { Energy-saving-development } \\
\text { funding awareness }\end{array}$ & $\begin{array}{l}\text { VAR074, VAR076, } \\
\text { VAR078 }\end{array}$ \\
\hline BEHAVE & Energy consumption behavior & $\begin{array}{l}\text { VAR075, VAR077, } \\
\text { VAR079 }\end{array}$ \\
\hline KWHUSE & $\begin{array}{l}\text { Energy usage in kilo-watt- } \\
\text { hours }\end{array}$ & KWH \\
\hline \multicolumn{3}{|c|}{ B. DESCRIPTOR CLUSTERS: } \\
\hline CLIMGEO & $\begin{array}{l}\text { Climatic/geographic } \\
\text { environment }\end{array}$ & GEOAREA \\
\hline TYPDWEL & Type of dwelling & $\begin{array}{l}\text { VAR005 } \\
\text { VAR469, VAR470 } \\
\text { VAR47I }\end{array}$ \\
\hline RENTOWN & $\begin{array}{l}\text { Status of the resident } \\
\text { in terms of renter/owner }\end{array}$ & VAR008 \\
\hline DEMOG & Demographic cluster & $\begin{array}{l}\text { VAR318, VAR360 } \\
\text { VAR362, VAR364 }\end{array}$ \\
\hline INSUL & $\begin{array}{l}\text { Level of insulation of } \\
\text { the residence }\end{array}$ & VAR133, VARI41 \\
\hline
\end{tabular}


CHAPTER IV

RESULTS

This chapter presents the results of the segment congruence analysis of PNWRES data. First, the research questions for Phase I are addressed. In this phase the segmentation variables are analyzed to:

1. Determine the existence and amount of the total mutual association among segmentation variables.

2. Identify the variable(s) with the highest contribution toward the total mutual association.

3. Identify the variable(s) with the highest contribution toward the joint variability in the system of segmentation variables.

4. Identify the variable(s) which result in the highest amount of reduction in the uncertainty of the system of segmentation variables.

5. Identify the variable(s) with the greatest number of significant dyadic relations with the other variables.

Phase II, the selection and analysis of the distinguished segmentation variable, is addressed next. The other segmentation variables are used as descriptor 
variables in one set of analyses, while five exogenous descriptor variables are used in another, in order to:

1. Identify the distinguished (i.e., the best) segmentation variable.

2. Assess the changes in the reduction of uncertainty in the distinguished variable, due to the descriptor variables, under different collapsing schemes.

3. Prioritize the contributions of the descriptor variables to the determination of the distinguished variable.

4. Analyze the structure of the distinguished variable and all of the descriptor variables using dependency analysis.

The energy usage variable (KWHUSE) is of particular importance to the. BPA decision makers, as well as the local utilities. Therefore, this variable will be included as a distinguished variable in addition to the analytically selected distinguished variable, should they not be the same.

In order to provide a comparative base with the classical segment congruence analysis, Phase II is first implemented using the endogenous variables (i.e., other segmentation variables) as descriptor variables, with the a priori selected, electricity consumption as the distinguished variable. Next, the analytically derived distinguished variable is analyzed. (It will be seen that energy related behavior is selected analytically.) 
Phase II is then repeated using exogenous descriptor variables to identify the distinguished variable and proceed with its analysis. (Here electricity consumption is selected analytically.) Figure 12 shows the flow of these analyses:

\section{PHASE I:}

A) ASSESS THE EXISTENCE AND AMOUNT OF MUTUAL ASSOCIATIONS AMONG SEGMENTATION VARIABLES (RESEARCH QUESTION \#1).

B) IDENTIFY CANDIDATE FOR THE DISTINGUISHED BASE THROUGH THE FOUR METHODS PROPOSED (RESEARCH QUESTION \#2 THRU \#5)

PHASE II WITH ENDOGENOUS VARIABLES:

A) A PRIORI, SEIECT ELECTRICITY CONSUMPTION, KWEUSE, AS THE DISTINGUISHED VARIABLE. THEN, ANALYZE KWHUSE USING OTHER SEGMENTATION VARIABLES AS THE DESCRIPTOR

VARIABLES (RESEARCK QUESTIONS \#I THRU \#4).

B) IDENTIFY THE DISTINGUISHED SEGMENTATION VARIABLE, ANAIYTICAILY, USING THE OTHER SEGMENTATION VARIABLES AS DESCRIPTOR VARIABLES (RESEARCH QUESTION \#I).

C) ANALYZE THE DISTINGUISHED VARIABIE OBTAINED IN (B), USING THE OTHER SEGMENTATION VARIABLES AS DESCRIPTOR VARIABLES (RESEARCH QUESTIONS \#2 THRU \#5).

PHASE II WITH EXOGENOUS VARIABLES:

A) IDENTIFY THE DISTINGUISHED SEGMENTATION VARIABLE, ANALYTICALLY, USING EXOGENOUS DESCRIPTOR VARIABLES (RESEARCH QUESTION \#I).

B) ANALYZE THE DISTINGUISHED SEGMENTATION VARIABLE, SEIECTED IN (C), USING THE EXOGENOUS VARIABLES AS THE DESCRIPTOR VARIABLES (RESEARCH QUESTIONS \#2 THRU \#5).

Figure 1.2. Condensed flow chart of data analysis. 


\section{ANALYSIS OF THE SEGMENTATION VARIABLES}

In addition to assessing the mutual association among the segmentation variables, this section identifies a set of candidate segmentation variables using various methods. In cases where a variable is selected as the candidate base by two different methods, as a convention, the second best variable will be designated as the candidate. This will ensure that each method will produce a new candidate. The logic for this convention relates to the fact that segment congruence analysis is ideally performed on a large number of variables, therefore, retaining at least four variables for further analyses is reasonable.

Existence and Degree of Mutual Association Among Segmentation Variables

First a set of segmentation variables (or clusterings) are introduced. Table XI in chapter III presents these variables, and Table $x$ Iists the variables selected from the PNWRES data (see Appendix IV for a complete list of variables). Chapter III also describes procedures used to obtain and cluster all of the variables used in this study. Table XII reproduces the listing of the segmentation variables for ease of reference. 
TABLE XII

IIST OF THE SEGMENTATION VARIABLES

\begin{tabular}{llllc}
\hline $\begin{array}{l}\text { Variable } \\
\text { (Abbreviation) }\end{array}$ & Description & \# of classes \\
\cline { 1 - 1 } GENATT & (G) & General Attitudes & & 4 \\
ENRATT & (E) & Energy Related Attitudes & 5 \\
PERCEPT & (P) & Energy Related Perceptions & & 4 \\
AWARE & (A) & Energy Related Awareness & 3 \\
BEHAVE & (B) & Energy Related Behavior & 2 \\
KWHUSE & (R) & Electricity Consumption & 5 \\
& & &
\end{tabular}

The analyses in Phase I will use the above segmentation variables. The first research question involves the assessment of mutual association among these variables.

Research Question \#1. The hypothesis tested for the mutual association assessment is as follows:

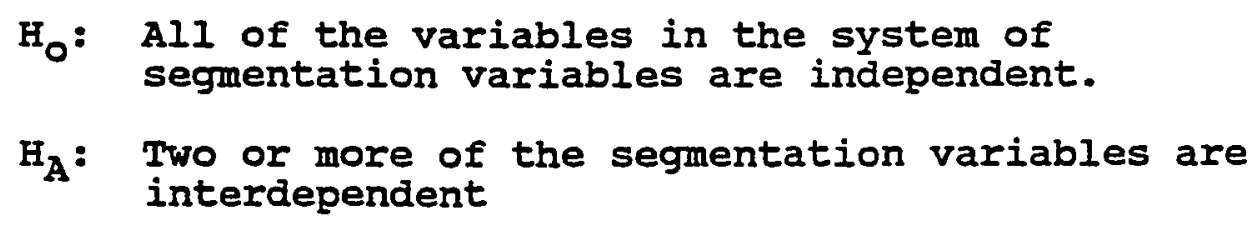

Table XIII presents the results of 1 ) the independence model based on the general log-linear approach, and 2) the transmission-based analysis of the mutual association among the segmentation variables: 
TABLE XIII

ASSESSMENT OF THE MUTUAL ASSOCIATION

Variables: GENATT (4), ENRATT (5), PERCEPT (4), AWARE (3), BEHAVE (2), KWHUSE (5),

where; $(\mathrm{n})=$ Corresponding variable has $\mathrm{n}$ Classes.

IOG-IINEAR APPROACH

Model: [GENATT\} \{ENRATT\} \{PERCEPT \} \{AWARE\} \{BEHAVE\} \{KWHUSE\}

$\frac{I^{2} L I}{3150.14} \frac{d f}{2382} \frac{p}{=0} \frac{N}{3335}$

\section{INFORMATION THEORETIC APPROACH}

Model : T (GENATT : ENRATT : PERCEPT : AWARE : BEHAVE : KWHUSE)

\begin{tabular}{|c|c|c|c|c|c|}
\hline $\mathbf{T}$ & $\mathrm{T} / \mathrm{T}_{\mathrm{MAX}}$ & $L^{2} I L$ & $d f$ & $\mathbf{p}$ & $\mathbf{N}$ \\
\hline 0.6814 & .0765 & 3150.14 & 2382 & $=0$ & 3335 \\
\hline
\end{tabular}

Examining Table XIII reveals that both the general log-linear model and the information theoretic model reject the null hypothesis with likelihood-ratio chi-square of 3150.14, $d f=2382$, and $p=0$. The transmission measure, $T$, of .6814 , and $T / T_{\max }$ of .0765 indicate that the amount of 
overlap is relatively small (but highly significant, statistically).

Thus, it is concluded that general attitudes, energy related attitudes, perceptions, awareness, and behavior, and energy consumption are mutually associated. This indicates that knowledge about the class of one or more of the variables reveals information about the classes of the other variables.

The remaining research questions in this phase, aim at determining which variables are likely candidates for a distinguished segmentation variable. Four different methods are proposed: Contribution of each segmentation variable to the (a) total mutual association, (b) the joint variability in the system, and (c) total uncertainty in the system; and (4) the number of significant (dyadic) relations between variables associated with each candidate variable.

Identification of Candidates for the Distinguished Variables Research Question \#2. Following is the list of the hypotheses tested in order to assess contribution of individual variables to the total mutual association among the segmentation variables:

1) $H_{0}$ : GENATT does not contribute toward the total mutual association in the system of segmentation variables.

$\mathrm{H}_{\mathrm{A}}$ : GENATT contributes toward the total mutual association in the system of segmentation variables. 
2) $\mathrm{H}_{0}$ : ENRATT does not contribute toward the total mutual association in the system of segmentation variables.

$\mathrm{H}_{\mathrm{A}}$ : ENRATT contributes toward the total mutual association in the system of segmentation variables.

3) $H_{0}$ : PERCEPT does not contribute toward the total mutual association in the system of segmentation variables.

$\mathrm{H}_{\mathrm{A}}$ : PERCEPT contributes toward the total mutual association in the system of segmentation variables.

4) $H_{0}$ : AWARE does not contribute toward the total mutual association in the system of segmentation variables.

$\mathrm{H}_{\mathrm{A}}$ : AWARE contributes toward the total mutual association in the system of segmentation variables.

5) Ho: BEHAVE does not contribute toward the total mutual association in the system of segmentation variables.

$\mathrm{H}_{\mathrm{A}}$ : BEHAVE contributes toward the total mutual association in the system of segmentation variables.

6) Ho: KWHUSE does not contribute toward the total mutual association in the system of segmentation variables.

$\mathrm{H}_{\mathrm{A}}$ : KWHUSE contributes toward the total mutual association in the system of segmentation variables.

Table XIV shows the results of the log-linear onevariable-independence models and their information theoretic equivalent models: 
TABLE XIV

ASSESSMENT OF THE CONTRIBUTION OF EACH VARIABLE TO THE MUTUAL ASSOCIATION

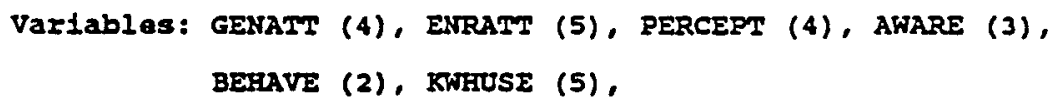

\begin{tabular}{|c|c|c|c|}
\hline $\begin{array}{l}\text { (1) } \\
\text { (i) }\end{array}$ & $\begin{array}{l}\text { (2) } \\
L^{2} L I\end{array}$ & $\begin{array}{l}\text { (3) } \\
\text { dE }\end{array}$ & $\begin{array}{r}\text { (4) } \\
p\end{array}$ \\
\hline GENATY & 2884.37 & 2340 & $=0$ \\
\hline ENRATT & 3013.86 & 2330 & $=0$ \\
\hline PERCEPT & 2861.70 & 2340 & $=0$ \\
\hline AKARE & 1269.70 & 2352 & 1 \\
\hline BEHAVE & 1279.92 & 2366 & 1 \\
\hline KWFUSE & 306889 & 2330 & $=0$ \\
\hline
\end{tabular}

INFORMATION THEORETIC APPROACH

Models: E.G.,

$\Phi_{1} T=T_{\text {GENATT }}$ (ENRATT: PERCEPT: AWARE : BEHAVE: KWHOSE)

\begin{tabular}{|c|c|c|c|c|c|}
\hline $\begin{array}{l}\text { (1) } \\
\text { Base } \\
\text { (i) }\end{array}$ & $\begin{array}{l}(2) \\
T_{i}\end{array}$ & $\begin{array}{c}\text { (3) } \\
I-T_{i} / T\end{array}$ & $\begin{array}{l}(4) \\
L^{2} I T\end{array}$ & $\begin{array}{l}\text { (5) } \\
d f\end{array}$ & $\begin{array}{l}\text { (6) } \\
p\end{array}$ \\
\hline GENATI & 0.6239 & 0.0844 & 2884.29 & 2340 & $=0$ \\
\hline ENRATT & 0.6519 & 0.0433 & 3013.74 & 2330 & $=0$ \\
\hline PERCEPT & 0.6190 & 0.0916 & 2861.64 & 2340 & $=0$ \\
\hline AWARE & 0.2747 & 0.5969 & 1269.84 & 2352 & 1 \\
\hline BEHAVE & 0.2768 & 0.5938 & 1279.55 & 2366 & 1 \\
\hline KWTUSE & 0.6637 & 0.0250 & 3068.29 & 2330 & $=0$ \\
\hline
\end{tabular}


The top part of Table XIV (i.e., the log-linear approach), includes the segmentation variables under the consideration, column 1 , and their corresponding likelihood ratio chi-square, degrees of freedom, and risk levels in columns 2, 3, and 4, respectively. The bottom part of this table, on the other hand, presents the information theoretic models equivalent to the log-linear models.

It is seen that both models generate identical statistics, however, the information theoretic model generates a measure of the amount contribution (i.e., $T_{i}$ in column 2), which is not given in the log-linear approach. Also, column 3 gives a normalized measure of the amount of contribution toward the joint variability in the system (1$T_{i} / T$ ) which has a maximum of one (i.e., when all of the association is due the variable under the consideration), and a minimum of zero (i.e., when the variable under investigation is independent of others).

Columns 4, 5, and 6 present the likelihood-ratio chisquare, degrees of freedom, and the risk levels for each model. ' $\mathrm{L}^{2} I T$ is calculated as $2 \mathrm{~N} \log (2) \mathrm{T}$, and degrees of freedom are calculated based on the formula given by Broekstra (1981) and reproduced in the methodology chapter. These calculations give the same values as those produced by the log-linear model. The differences in the decimals are attributed to the fact that cells with sampling zeros had to 
be given a negligible value (i.e., IE-20) in the log-linear model, as the proportional fitting method used would generate incorrect statistics without such adjustment.

In order to select the best variable the magnitude of the likelihood-ratio chi-square was used for the log-linear approach, and the normalized measure in column 3 (i.e., I$\left.T_{i} / T\right)$ was used for the information theoretic approach. Based on both approaches, the energy related awareness variable, $A W A R E$, is selected as the candidate for the distinguished segmentation variable.

Research Question \#3. This research question assesses the contribution of each of the segmentation variables toward the joint variability in the system of segmentation variables. In information theoretic terms, this assessment leads to the identification of the variable(s) with the highest contribution toward the total systematic entropy in the system.

Following is the list of the hypotheses tested in order to assess the second research question in this phase:

1) $\mathrm{H}_{0}$ : GENATT does not contribute toward the joint variability in the system of segmentation variables.

$\mathrm{H}_{\mathrm{A}}$ : GENATT contributes toward the joint variability in the system of segmentation variables.

2) $H_{0}:$ ENRATT does not contribute toward the joint variability in the system of segmentation variables. 
$\mathrm{H}_{\mathrm{A}}$ : ENRATT contributes toward the joint variability in the system of segmentation variables.

3) $\mathrm{H}_{\mathrm{O}}$ : PERCEPT does not contribute toward the joint variability in the system of segmentation variables.

$\mathrm{H}_{\mathrm{A}}$ : PERCEPT contributes toward the joint variability in the system of segmentation variables.

4) $\mathrm{H}_{0}$ : AWARE does not contribute toward the joint variability in the system of segmentation variables.

$\mathrm{H}_{\mathrm{A}}$ : AWARE contributes toward the joint variability in the system of segmentation variables.

5) $H_{0}$ : BEHAVE does not contribute toward the joint variability in the system of segmentation variables.

$\mathrm{H}_{\mathrm{A}}$ : BEHAVE contributes toward the joint variability in the system of segmentation variables.

6) $\mathrm{H}_{0}$ : KWHUSE does not contribute toward the joint variability in the system of segmentation variables.

$\mathrm{H}_{\mathrm{A}}$ : KWHUSE contributes toward the joint variability in the system of segmentation variables.

As mentioned previously, the single-variable independence model in the log-linear approach has been misinterpreted as assessing the contribution of individual variables toward the total mutual association. It has been shown in the present study that this model assesses instead the contribution of individual variables to the joint variability in the multivariate system. 
Table XV shows the results, based on this new interpretation of the log-linear one-variable-independence models, and their information theoretic equivalent models: The log-linear portion in Table XV includes the segmentation variables under the consideration, column 1 , and their corresponding likelihood ratio chi-square, degrees of freedom, and risk levels in columns 2,3 , and-4, respectively. The bottom part of this table, on the other hand, presents the information theoretic equivalent of this model.

Again, both models generate identical statistics, however, the information theoretic model generates a measure of the amount contribution (i.e., s-s $s_{i}$ in column 2), which is not given in the log-linear approach. Also, column 3 gives the normalized measure of the amount of contribution toward the joint variability in the system.

In order to determine which variable contributes the most toward the joint variability in the system, Green and Carmone's (1977) logic is followed. A model which gives a lower $I^{2}$ fits the data better than a model that gives a higher $L^{2}$. Therefore,. for the single-variable-independence model, the model that gives the highest $\mathrm{L}^{2}$ should be selected as the model which fits the data the least. 
TABLE XV

ASSESSMENT OF THE CONTRIBUTION OF EACY

VARIABLE TO THE JOINT VARIABILITY IN THE SYSTEM OF SEGMENTATION VARIABLES

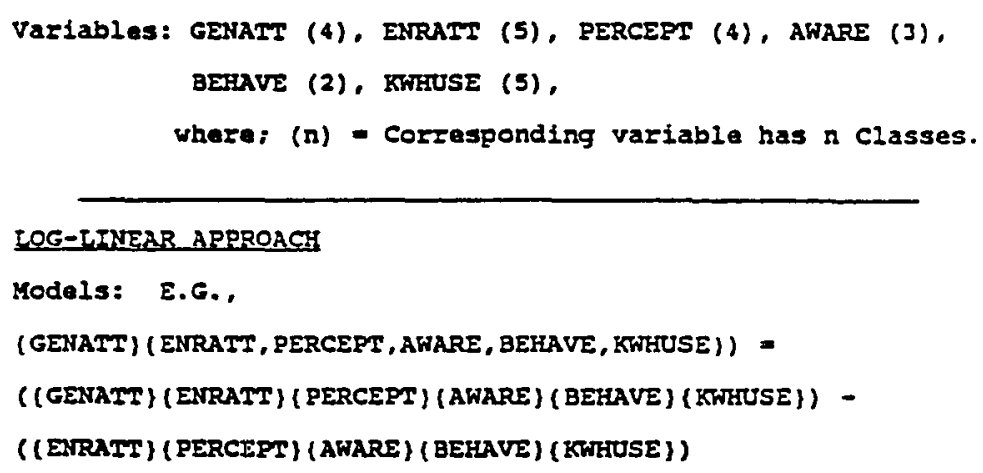

\begin{tabular}{|c|c|c|c|}
\hline $\begin{array}{l}\text { (1) } \\
\text { Base } \\
\text { (1) }\end{array}$ & $\begin{array}{l}(2) \\
\tau^{2} \tau L\end{array}$ & $\begin{array}{l}\text { (3) } \\
\text { de }\end{array}$ & $\begin{array}{l}\text { (4) } \\
\text { p }\end{array}$ \\
\hline GENATT & 858.53 & 1797 & $=0$ \\
\hline ENRATT & 809.31 & 1926 & $=0$ \\
\hline EERCEPT & 861.31 & 1797 & $=0$ \\
\hline AWARE & 2320.77 & 1598 & $=1$ \\
\hline BEHAVE & 2111.75 & 1199 & $=1$ \\
\hline KWTUSE & 724.45 & 1916 & $=0$ \\
\hline
\end{tabular}

\section{INFORMATION THEORETIC ARPROACY}

Models: E.G.,

$\Phi_{1} S$ - S (GENATT: ENRATT : PERCEPT : AWARE: BEHAVE: KWHUSE) -

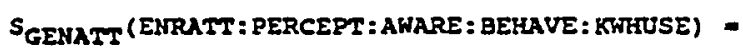
T (GENATT: ENRATT: PERCEPT: AWARE: BEHAVE: KWHUSE) T (ENRATT : PERCEPT : AWARE: BEHAVE: KWTUSE)

\begin{tabular}{|c|c|c|c|c|c|}
\hline $\begin{array}{l}\text { (1) } \\
\text { Base } \\
\text { (i) }\end{array}$ & $\begin{array}{r}\text { (2) } \\
s-s_{i}\end{array}$ & $\begin{array}{c}\text { (3) } \\
1-5_{i} / S\end{array}$ & $\begin{array}{l}(4) \\
I^{2} I I\end{array}$ & (5) & ? \\
\hline GENATT & 0.1857 & 0.1893 & 858.53 & 2797 & $=0$ \\
\hline ENRATT & 0.1751 & 0.1785 & 809.31 & 1916 & $=0$ \\
\hline PERCEPT & 0.1863 & 0.1899 & 862.31 & 2797 & $=0$ \\
\hline AWARE & 0.5020 & 0.5117 & 2320.77 & 1598 & $=:$ \\
\hline SEHAVE & 0.4568 & 0.4656 & 2121.75 & $: 199$ & $=:$ \\
\hline EWHUSE & 0.1567 & 0.1597 & 224.45 & $: 9: 6$ & $=0$ \\
\hline
\end{tabular}


Based on the above reasoning, both the log-linear and the information theoretic approach would result in the choice of the energy related awareness (AWARE) as a candidate for the distinguished segmentation variable. Based on the convention established at the beginning of this section, however, the second best variable (i.e., energy use behavior, BEHAVE) will be selected (because, AWARE has already been selected). Therefore, the variable BEHAVE is designated as the candidate segmentation variable.

Research Question \#4. This research question examines the contribution of each variable toward the overall uncertainty in the system. First, the significance of each contribution is tested, and next, the amount of contribution is used to identify a candidate for the distinguished variable.

Following is the list of hypotheses tested to address the significance of each variable's contribution:

1) $H_{0}$ : GENATT does not contribute toward the overall uncertainty in the system of segmentation variables.

$\mathrm{H}_{\mathrm{A}}$ : GENATT contributes toward the overall uncertainty in the system of segmentation variables.

2) $H_{0}:$ ENRATT does not contribute toward the overall uncertainty in the system of segmentation variables.

$\mathrm{H}_{\mathrm{A}}$ : ENRATT contributes toward the overall uncertainty in the system of segmentation variables. 
3) $H_{0}$ : PERCEPT does not contribute toward the overall uncertainty in the system of segmentation variables.

$\mathrm{H}_{\mathrm{A}}$ : PERCEPT contributes toward the overall uncertainty in the system of segmentation variables.

4) $\mathrm{H}_{\mathrm{O}}$ : AWARE does not contribute toward the overall uncertainty in the system of segmentation variables.

$\mathrm{H}_{\mathrm{A}}$ : AWARE contributes toward the overall uncertainty in the system of segmentation variables.

5) $\mathrm{H}_{0}$ : BEHAVE does not contribute toward the overall uncertainty in the system of segmentation variables.

$\mathrm{H}_{\mathrm{A}}$ : BEHAVE contributes toward the overall uncertainty in the system of segmentation variables.

6) $H_{0}$ : KWHUSE does not contribute toward the overall uncertainty in the system of segmentation variables.

$\mathrm{H}_{\mathrm{A}}$ : KWHUSE contributes toward the overall uncertainty in the system of segmentation variables.

In Chapter 3, it was argued that the application of the log-linear approach to research questions which deal with the uncertainty in a multivariate system is not necessary (i.e., due to eventual necessity to resort to the entropy measure and the lack of availability of a known computer program to perform such analysis). Therefore, the log-linear approach will not be applied for this question as well as other questions dealing with overall system uncertainty. Table XVI presents the results of the information theoretic analysis: 
TABLE XVI

ASSESSMENT OF THE CONTRIBUTION OF EACH VARIABLE TO THE TOTAL UNCERTAINTY IN THE SYSTEM OF

SEGMENTATION VARIABLES

Models: E.G.,

$\Phi_{1} \mathrm{H}=\mathrm{H}$ (GENATT, ENRATT, PERCEPT, AWARE, BEHAVE, KWHUSE) H(GENATT)

INFORMATION THEORETIC APPROACH

(1)

i

GENATT $\quad-$

ENRATT 2.2413

PERCEPT $\quad 1.3687$

AWARE $\quad 1.0131$

BEHAVE $\quad 0.6186$

KWHUSE $\quad 1.4256$
(3)

$\mathbf{H}_{\mathbf{i}}$

5.9859

5.3017

6.1743

6.5300

6.9245

6.1175
(4)

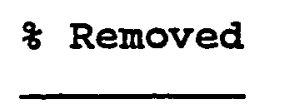

20.64

29.71

18.15

13.43

8.20

18.90
(5)

(6)

(7)

$\begin{array}{rcc}I^{2} & \text { d.f. } & p \\ 2047.66 & 3 & 0 \\ 372.77 & 4 & 0 \\ 2918.68 & 3 & 0 \\ 2643.89 & 2 & 0 \\ 1763.32 & 1 & 0 \\ 4143.99 & 4 & 0\end{array}$

In Table XVI, Column 1, lists the variables whose contribution to the overall uncertainty in the system is being investigate. Column $2, H(i)$, is the uncertainty of each variable $i$. Column $3, \mathrm{H}_{i}$, is the uncertainty in the overall system given the variable $i$ (i.e., the remaining uncertainty). Column 4, \& removed, is $H(i) /$ (total uncertainty in the system). Column 5 , is the $\mathrm{I}^{2}$ calculated 
based on the relationship between uncertainty and likelihood-ratio chi-square (i.e., $I^{2}=2 \mathrm{~N} \log (2) \mathrm{H}(\mathrm{i})-2 \mathrm{~N} \operatorname{logA}$, where $N$ =total number of observations, $H(i)=$ entropy of variable $i$, and $A=$ number of states of variable $i$ ). For instance, the $\mathrm{I}^{2}$ for $i=G E N A T T$ is calculated as:

$$
I^{2}=2 * 3335 * \log (2) * 1.5571-2 * 3335 * \log (4)=2047.66
$$

Columns 6 and 7 list the degrees of freedom and the risk level for each variable under consideration.

Based on the $I^{2}$, all of these variables contribute significantly to the overall uncertainty in the system $(p=0$ for rejecting the null hypotheses for all variables). Beyond this result no other interpretations can be made as the degrees of freedom differ in each case and therefore the $I^{2}$ are not directly comparable.

Judging by the percent of contribution of each variable, the energy related attitudes variable, ENRATT is selected as the candidate for the distinguished segmentation variable (ENRATT contributes $29.71 \%$ to the overall uncertainty).

Research ouestion \#5. In order to address this research question, bivariate associations were investigated for all possible two variable combinations, using both methods. The likelihood-ratio chi-square was then used to assess the significance of each bivariate model. For this purpose, a two dimensional table was constructed of the 
likelihood-ratio chi-squares and, for the information theoretic approach, the amounts of bivariate transmission. To assess the significance of the association within each two-variable combination, hypotheses similar to the following were tested:

$$
\begin{aligned}
& \mathrm{H}_{0}: \text { The two variables being considered are } \\
& \text { independent. } \\
& \mathrm{H}_{\mathrm{A}} \text { : The two variables are interdependent }
\end{aligned}
$$

Table XVII presents the two bivariate tabulations. One based on the likelihood-ratio chi-squares derived from the log-linear models, and another based the information theoretic bivariate transmissions and likelihood-ratio chisquares derived from these transmissions:

The bivariate table for the log-linear approach includes the likelihood-ratio chi-square, the degrees of freedom, and the risk value for each bivariate combination of segmentation variables. These figures appear in the upper triangle of the top bivariate table. The information theoretic approach produces the same bivariate table, with identical upper triangle, but with the addition of measures of association (i.e., bivariate transmissions), presented in the lower triangle. 
TABLE XVII

ASSESSMENT OF THE NUMBER OF SIGNIFICANT DYADIC RELATIONS

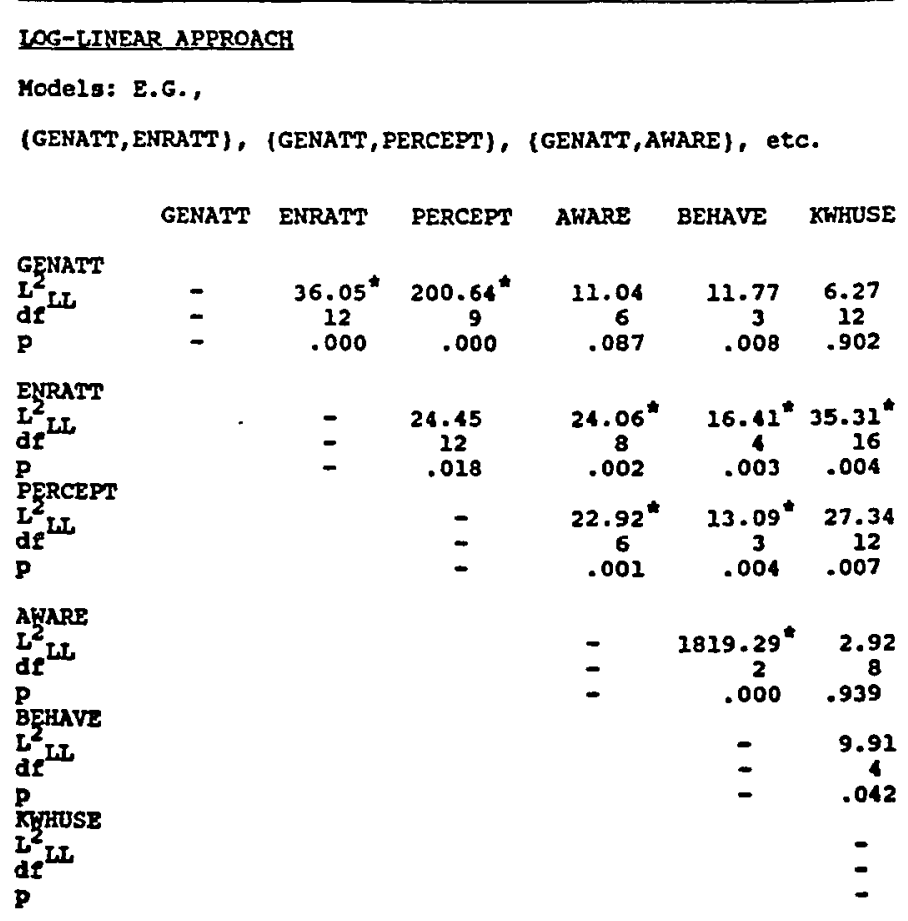

\section{INPORYATON THEORETTC APPROACH}

Hodele: E.G..

T(GENATT:ENRATI)，T(GENATT: PERCEPT)，T(GENATT:AWARE), atc.

GENATT ENRATT PERCEPT AWARE BEHAVE KWHUSE

\begin{tabular}{|c|c|c|c|c|c|c|}
\hline $\begin{array}{l}\text { GPNATT } \\
\mathrm{L}^{2} \mathrm{WT} \\
\mathrm{de} \\
\mathrm{p}\end{array}$ & $\overline{-}$ & $\begin{array}{c}36.05^{\star} \\
12 \\
.000\end{array}$ & $\begin{array}{c}200.64 \\
9 \\
.000\end{array}$ & $\begin{array}{r}11.04 \\
6 \\
.087\end{array}$ & $\begin{array}{c}11.77 \\
3 \\
.008\end{array}$ & $\begin{array}{l}6.27 \\
12 \\
.902\end{array}$ \\
\hline $\begin{array}{l}\text { EXRATT } \\
\text { LELI } \\
\text { de } \\
\text { p }\end{array}$ & 0.0078 & $=$ & $\begin{array}{c}24.45 \\
12 \\
.018\end{array}$ & $\begin{array}{c}24.06^{\star} \\
8 \\
.002\end{array}$ & $\begin{array}{c}16.41 \\
4 \\
.003\end{array}$ & $\begin{array}{r}35.31^{*} \\
16 \\
.004\end{array}$ \\
\hline $\begin{array}{l}\text { PFRCEPT } \\
L^{2} \mathrm{LL} \\
\mathrm{de} \\
\mathrm{p}\end{array}$ & 0.0434 & 0.0053 & $=$ & $\begin{array}{c}22.92^{\star} \\
6 \\
.001\end{array}$ & $\begin{array}{c}13.09 \\
3 \\
.004\end{array}$ & $\begin{array}{l}27.34 \\
12 \\
.007\end{array}$ \\
\hline 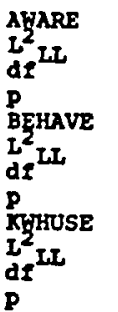 & 0.0025 & 0.0035 & 0.0028 & $\begin{array}{c}= \\
= \\
0.3935\end{array}$ & $\begin{array}{c}1819.29^{\star} \\
2 \\
.000 \\
= \\
= \\
0.0021\end{array}$ & $\begin{array}{c}2.92 \\
9 \\
.939 \\
9.91 \\
4 \\
.042 \\
- \\
- \\
-\end{array}$ \\
\hline
\end{tabular}


Judging on the basis of significance of the bivariate likelihood-ratio chi-square, with the confidence level at 0.005 , the significant bivariate associations are designated with "*" in both tables. It is seen that the energy related attitudes variable, ENRATT, has the highest number of bivariate associations. However, this variable has already been selected, therefore, the energy related perception variable, PERCEPT, is selected as the candidate variable. Note that, there are two other variables (i.e., AWARE and BEHAVE) with the same number of significant bivariate associations, which have already been selected. In case of a tie between two (or more) variables, both (all) of which have not been selected yet, the confidence level is set at progressively narrower levels until one of the variables prevails. In the this application, however, this was not necessary and PERCEPT was selected as the candidate for the distinguished variable.

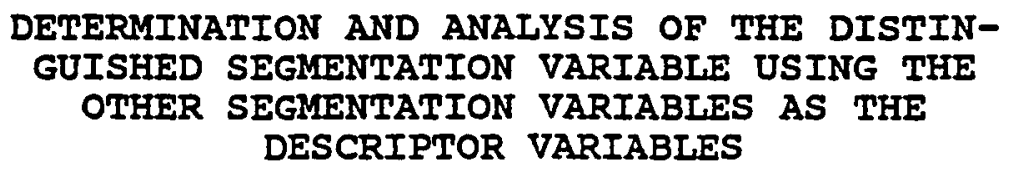

In Phase I, four candidates for the distinguished segmentation variable were identified. These variables include ENRATT, PERCEPT, AWARE, BEHAVE AND KWHUSE. Phase II continues the process of segment congruence analysis by assessing predictability of various segmentation variables 
by a set of descriptor variables (i.e., either endogenous, using other segmentation variables, or exogenous, using other descriptor variables, such as demographics, socioeconomics, etc.).

First, electricity consumption, KWHUSE, is selected as the distinguished variable, a priori. This variable is then analyzed using the other segmentation variables (i.e., endogenous variables) as descriptor variables. This will virtually replicate Green and Carmone (1977) analysis with the addition of other research questions introduced in the present study.

The analyses will then proceed by identifying and analyzing the distinguished segmentation variable using the endogenous variables first. Then, in the section 5.3 , a set of exogenous variables will be used in order to identify and analyze the distinguished variable.

\section{Electricity Consumption as the Distinquished segmentation} Variable (A Priori)

Research Question \#1. Electricity consumption, KWHUSE, was identified by the BPA and PP\&L executives as the most significant variable. In the context of market segmentation, also, this variable is of particular interest, as it is the variable by which the heavy user group can be distinguished from the light user. Therefore, KWHUSE is selected, a priori, as the distinguished segmentation variable in part (A) of this section. 
Research Question \#2. This research question aims at measuring the amount of reduction in uncertainty in the distinguished variable when different classes are reclustered (e.g., into dichotomies of a cluster of interest and all other clusters). The segmentation variable (here, energy consumption, KWHUSE) is examined with respect to three different groupings: the low-consumption group (classes 1 \& 2 of KWHUSE), the medium-consumption group (class 3 of KWHUSE), and the high-consumption group (classes 4 \& 5 of KWHUSE). Table XVIII shows the results of these analyses.

In Table XVIII, the logit analysis for each reclassification scheme, column 1, produces the goodness-offit likelihood ratio chi-square, column 2 , and its associated degrees of freedom, and risk level, columns 3 and 4, respectively. Additionally, the logit model provides the measures of entropy and concentration, which are measures of association between the dependent variable and independent variables (refer to the chapter II, literature search, pp. 35-36). These measure are shown in columns 5 and 6 , respectively.

Column 7 shows the ranking of different reclassifications based on their measure of entropy. 
TABLE XVIII

PREDICTABILITY OF A PARTICULAR CLASS VS. ALI OTHER CLASSES OF THE DISTINGUISHED VARIABLE

IOG-LINEAR APPROACH (THE LOGIT MODEI)

\begin{tabular}{|c|c|c|c|c|c|c|}
\hline $\begin{array}{l}\text { (I) } \\
\text { Reclassi- } \\
\text { fication } \\
\text { Scheme for } \\
\text { KWHUSE }\end{array}$ & $I^{2} I I$ & (3) & (4) & $\frac{\text { Ana. of }}{\text { Entropy }}$ & $\frac{\text { Disper. }}{\text { Concent }}$ & Rank \\
\hline $\begin{aligned} \text { Class } 1 \& 2 & =1 \\
\text { Others } & =2\end{aligned}$ & 218.45 & 466 & 1 & 0.0180 & 0.0122 & 2 \\
\hline $\begin{array}{l}\text { Class } 3=1 \\
\text { Others }=2\end{array}$ & 250.02 & 466 & 1 & 0.0051 & 0.0069 & 3 \\
\hline $\begin{aligned} \text { Class } 4 \& 5 & =1 \\
\text { Others } & =2\end{aligned}$ & 212.16 & 466 & 1 & 0.0111 & 0.0134 & 1 \\
\hline
\end{tabular}

INFORMATION THEORETIC APPROACH

(I)

Reclassi-

fication

scheme for

KWHUSE
(2)

Uncert of
Uncert. of seg.Var. seg. Var. given others H. (R)
(4)

Amount ( $\%$ )

Uncert.

Predicted Rank

$0.0554(6.45 \%) \quad 2$

0.8042

0.9085

$0.0590(6.10 \%) \quad 3$

Class $4 \& 5=1$

Class $3=1$
0.9675

0.5037

0.4474

$0.0563\left(11.18 \frac{8}{6}\right) \quad 1$

$$
\text { Others }=2
$$

0.5037 
Ranking based on only likelihood-ratio chi-square would also produce the same ranking, however, it is customary to rank on the basis of a measure of association rather than a measure of statistical significance. Furthermore, the measure of entropy was selected because it assesses the amount of total variability due to (or accounted for by) the independent variable. Based on this ranking, the high electricity consumption group is predicted better than the other reclassifications $\left(L^{2}=212.16\right)$ followed by the low and the medium-consumption groups $\left(L^{2}=218.45\right.$ and 250.02 , respectively). As can be seen here, in logit analysis, lower amounts of $\mathrm{I}^{2}$ indicated a better fit for the logit function than the higher ones (see Green and Carmone 1977). The information theory model, in Table XVIII, measures (in column 2) the uncertainty in each reclassification (as represented by the entropy of the variable), the uncertainty of that variable, given the descriptor variables, column 3 , the amount and the percent of uncertainty removed by the descriptor variables, column 4.

Column 5 gives the ranking of the predictive power based on the information theoretic model. The ranking is identical to that produced by the logit model. This is not a coincidence, since, the information theoretic model addresses the amount of variability remaining in the criterion variable data, with the knowledge of a set of independent variables. (This is reminiscent to the measure 
of entropy.) The entropy of the dependent variable knowing the independent variables, in essence measures the residuals in the logit model.

Therefore, it is concluded that the low-consumption group is best predicted by the descriptor variables. It is also seen that, the medium-consumption group is determined by the least amount by the other variables.

Research Question \#3. In order to prioritize the contributions of independent variables toward the determination of the energy usage variable, KWHUSE, the general log-linear model was re-configured and applied. The upper part of Table XIX, presents the results.

Abrahams and van Bueren's (1980) procedure was used for the ranking of the descriptor variables using information theory. The bottom part of Table XIX shows this ranking. The first step evaluates the bivariate marginal transmissions (here, ENRATT is selected as the variable with the highest bivariate transmission). Next, bivariate conditional (partial) transmission (on the condition that ENRATT is given/known), and so on.

This procedure produced the following ranking: ENRATT, PERCEPT, GENATT, AWARE, and BEHAVE. The two approaches produced the same ranking.This same procedure is used by conant (1982) in developing the dependency analysis. This technique is applied in Research Question \#4. 
TABLE XIX

PRIORITIZED EFFECT OF THE OTHER SEGMENTATION VARIABIES ON ENERGY CONSUMPTION

\begin{tabular}{|c|c|c|c|}
\hline Iog-Iinear Model & $I^{2} I I$ & de & $\mathbf{P}$ \\
\hline $\begin{array}{l}\{\mathrm{R}\}(\mathrm{G}\} \\
\{\mathrm{K}\}\{\mathrm{E}\} \\
(\mathrm{K}\}\{\mathrm{P}\} \\
\{\mathrm{R}\}\{\mathrm{A}\} \\
\{\mathrm{K}\}\{\mathrm{B}\}\end{array}$ & $\begin{array}{c}6.27 \\
35.31 \star \\
27.34 \\
2.92 \\
9.91\end{array}$ & $\begin{array}{r}12 \\
16 \\
12 \\
8 \\
4\end{array}$ & $\begin{array}{l}0.902 \\
0.004 \\
0.007 \\
0.939 \\
0.042\end{array}$ \\
\hline $\begin{array}{l}\{\mathrm{R}\}(\mathrm{EG}\} \\
\{\mathrm{K}\}\{\mathrm{ER}\} \\
\{\mathrm{R}\}\{\mathrm{EA}\} \\
\{\mathrm{K}\}\{\mathrm{EB}\}\end{array}$ & $\begin{array}{c}102.28 \\
110.86 \star \\
73.68 \\
59.29\end{array}$ & $\begin{array}{l}76 \\
76 \\
56 \\
36\end{array}$ & $\begin{array}{l}0.024 \\
0.006 \\
0.057 \\
0.009\end{array}$ \\
\hline $\begin{array}{l}\text { (K) }(\text { EPG }) \\
(\mathrm{R})(\text { EPA }) \\
(\mathrm{K})(\mathrm{EPB})\end{array}$ & $\begin{array}{l}278.95 * \\
228.93 \\
177.25\end{array}$ & $\begin{array}{l}316 \\
236 \\
156\end{array}$ & $\begin{array}{l}0.934 \\
0.617 \\
0.117\end{array}$ \\
\hline $\begin{array}{l}(\mathrm{K})(\mathrm{EPGA}) \\
(\mathrm{K})(\mathrm{EPGB})\end{array}$ & $\begin{array}{l}563.36 \star \\
437.19\end{array}$ & $\begin{array}{l}956 \\
642\end{array}$ & $\begin{array}{l}1.000 \\
1.000\end{array}$ \\
\hline (K) $($ EPGAB $)$ & $724.45 * 1$ & 1916 & 1.000 \\
\hline
\end{tabular}

INFORYATION THEORETIC APPROACH

\begin{tabular}{|c|c|c|c|c|c|}
\hline Model & $\mathbf{T}$ & $I^{2} I T$ & de & p & Cum $I^{2} I T$ \\
\hline $\begin{array}{l}T(K: G) \\
T(R: E) \\
T(K: P) \\
T(R: A) \\
T(R: B)\end{array}$ & $\begin{array}{l}0.0014 \\
0.0076 \\
0.0059 \\
0.0006 \\
0.0021\end{array}$ & $\begin{array}{r}6.47 \\
35.14 \\
27.28 \\
2.77 \\
9.71\end{array}$ & $\begin{array}{r}12 \\
16 \\
12 \\
8 \\
4\end{array}$ & $\begin{array}{l}\overline{0.902} \\
0.004 \\
0.004 \\
0.939 \\
0.042\end{array}$ & 35.14 \\
\hline $\begin{array}{l}T_{E}(K: G) \\
T_{E}(K: P) \\
T_{E}(K: A) \\
T_{E}(K: B)\end{array}$ & $\begin{array}{l}0.0145 \\
0.0163 \\
0.0083 \\
0.0052\end{array}$ & $\begin{array}{l}67.04 \\
75.36 \\
38.37 \\
24.04\end{array}$ & $\begin{array}{l}60 \\
60 \\
40 \\
20\end{array}$ & $\begin{array}{l}0.024 \\
0.006 \\
0.057 \\
0.009\end{array}$ & 110.50 \\
\hline $\begin{array}{l}T_{E P}(K: G) \\
T_{E P}(K: A) \\
T_{E P}(K: B)\end{array}$ & $\begin{array}{l}0.0364 \\
0.0255 \\
0.0144\end{array}$ & $\begin{array}{r}168.29 \\
217.89 \\
66.58\end{array}$ & $\begin{array}{r}240 \\
160 \\
80\end{array}$ & $\begin{array}{l}0.934 \\
0.617 \\
0.117\end{array}$ & 278.79 \\
\hline $\begin{array}{l}T_{E P G}(K: A) \\
T_{E P G}(K: B)\end{array}$ & $\begin{array}{l}0.0616^{\star} \\
0.0342\end{array}$ & $\begin{array}{l}284.80 \\
158.12\end{array}$ & $\begin{array}{l}640 \\
320\end{array}$ & $\begin{array}{l}1.000 \\
1.000\end{array}$ & 563.59 \\
\hline$T$ (EPGA $_{\text {ER: }}(\mathrm{B})$ & $0.0347^{\star}$ & 160.43 & 960 & 1.000 & 724.02 \\
\hline
\end{tabular}

PRIORITIZATION BASED ON BOTH APPROACHES:

H (KWEUSE) $=T$ (KWTUSE: ENRATT) + TE

+ TENRATT, PERCEPT (KWHUSE:GENATT)

+ TENRATT, PERCEPT, GENATT (KWEUSE: AWARE)

- IENRATT, PERCEPT, GENATT, AWARE (KWHUSE: BEHAVE)

- HENRATT, PERCEPT, GENATT, AWARE, BEHAVE (KWIUSE) 
Research question \#4. This research question requires an analysis of the dependency (Conant 1982) in the system of variables. Dependency analysis can be performed using results of the SYSENT program, which can treat an arbitrary number of variables and classes. First, a reduced (fourvariable) will be analyzed. Next, the total system of segmentation variables, in its original form will be assessed.

To construct the simplified model, the distinguished segmentation variable, energy consumption (KWHUSE) was included along with three top variables in the prioritization step. These variables were energy related attitudes, ENRATT, energY related perceptions, PERCEPT, and general attitudes, GENATT.

Dependency Analysis of the Four-Variable system of KWHUSE, ENRATT, PERCEPT, and GENATT. Table XX presents the dependency analysis results of the four-variable system of KWHUSE, ENRATT, PERCEPT, and GENATT.

In Table $x x$, column I gives the variable under consideration, column 2 gives the level of dependency, e.g., level $1(n=1)$ tests one variable dependencies, level 2. $(n=2)$ tests two-variable dependencies, etc. Column 3 gives the actual variables whose dependency to the variable in column $I$ is being assessed. 
TABLE XX

DEPENDENCY ANALYSIS OF THE FOUR-VARIABLE

SYSTEM OF GENATT, ENRATT, PERCEPT, AND KWHUSE

\begin{tabular}{|c|c|c|c|c|c|c|}
\hline (1) & (2) & (3) & (4) & (5) & (6) & (7) \\
\hline$i$ & n & $s_{i}$ & $T\left(i: S_{i}\right)$ & $T_{n} / H(i)$ & \& & $T(n)-T(n-1)$ \\
\hline $\mathbf{K}$ & $\begin{array}{l}1 \\
2 \\
3\end{array}$ & $\begin{array}{l}\mathbf{E} \\
\mathbf{E P} \\
\text { EPG }\end{array}$ & $\begin{array}{l}0.0076 \\
0.0239 \\
0.0603\end{array}$ & $\begin{array}{l}0.0053 \\
0.0168 \\
0.0423\end{array}$ & $\begin{array}{r}12.60 \\
39.64 \\
100.00\end{array}$ & $\begin{array}{l}0.0076 \\
0.0163 \\
0.0364\end{array}$ \\
\hline $\mathbf{E}$ & $\begin{array}{l}1 \\
2 \\
3\end{array}$ & $\begin{array}{l}\text { G } \\
\text { GK } \\
\text { GKP }\end{array}$ & $\begin{array}{l}0.0078 \\
0.0286 \\
0.0695\end{array}$ & $\begin{array}{l}0.0035 \\
0.0128 \\
0.0310\end{array}$ & $\begin{array}{r}11.22 \\
41.15 \\
100.00\end{array}$ & $\begin{array}{l}0.0078 \\
0.0208 \\
0.0409\end{array}$ \\
\hline P & $\begin{array}{l}1 \\
2 \\
3\end{array}$ & $\begin{array}{l}\mathbf{G} \\
\text { GE } \\
\text { GEK }\end{array}$ & $\begin{array}{l}0.0434 \\
0.0586 \\
0.0968\end{array}$ & $\begin{array}{l}0.0317 \\
0.0428 \\
0.0707\end{array}$ & $\begin{array}{r}44.83 \\
60.54 \\
100.00\end{array}$ & $\begin{array}{l}0.0434 \\
0.0125 \\
0.0382\end{array}$ \\
\hline $\mathbf{G}$ & $\begin{array}{l}1 \\
2 \\
3\end{array}$ & $\begin{array}{l}\mathbf{P} \\
\mathbf{P E} \\
\text { PEK }\end{array}$ & $\begin{array}{l}0.0434 \\
0.0611 \\
0.0975\end{array}$ & $\begin{array}{l}0.0279 \\
0.0392 \\
0.0626\end{array}$ & $\begin{array}{r}44.51 \\
62.67 \\
100.00\end{array}$ & $\begin{array}{l}0.0434 \\
0.0177 \\
0.0364\end{array}$ \\
\hline
\end{tabular}

Column 4 presents the bivariate transmissions between variables in column 1 and column 3 (variables in column 3 are taken as one aggregate variable). Column 5 gives a normalized measure of dependency (i.e., normalized by the entropy in the variable in column 1). Column 6 gives the percent of total dependency accounted for by level $n$. Finally, column 7 gives the amount dependency attributed to each additional level.

Using the information in Table $\mathrm{XX}$ and $\mathrm{a}$ threshold level of $80 \%$, the dependency structures for each variable 
are presented in Figure 13. Each graph includes the variables selected with respect to the threshold level for one of the variable (Figure 13, a thru d). Figure 13 (e) shows the combined structure constructed from the individual dependencies.

As mentioned in the methodology chapter, each variable selected carries the assumption that all variables higher in the ranking are present in the final structure and contribute indirectly to its measure of dependence (i.e., partial transmission). For example, in Figure 13 (a), GENATT contributes 0.0364 to KWHUSE if the relation KWHUSE \-> ENRATT $\longrightarrow->$ PERCEPT is present, otherwise, it contributes 0.0014 on its own.

Referring to Figure 13, the structure is constructed as KEP/EKP/EPG/GEP. However, KEP and EKP are the same and EPG and GEP are also identical. Therefore, the structure becomes KEP/EPG. This structure is presented Figure 13 (f). The interpretation of this structure may be stated as follows. The strongest dependency exists between general attitudes, GENATT, and energy related perceptions, PERCEPT (0.0434). GENATT also has a strong effect of energy related attitudes (0.0078). Energy related attitudes, ENRATT, and perceptions, PERCEPT, in turn are directly interdependent with electricity consumption, KWHUSE. 
Dependency Analysis of the Six-Variable System of KWHUSE, ENRATT, PERCEPT, GENATT, AWARE, and BEHAVE. The six variable system of segmentation variables can be analyzed through conant's method without any alterations in the number of variables or classes. Table XXI shows the dependency analysis results.

(a)

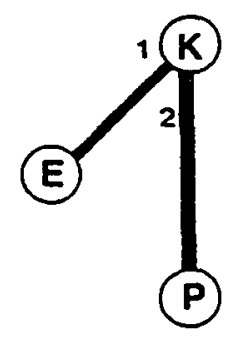

(G)

(d)

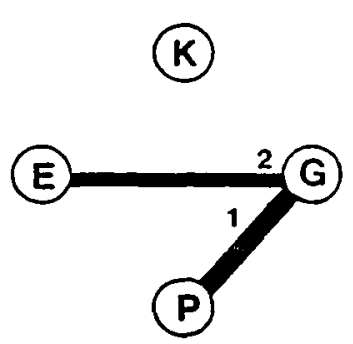

(b)

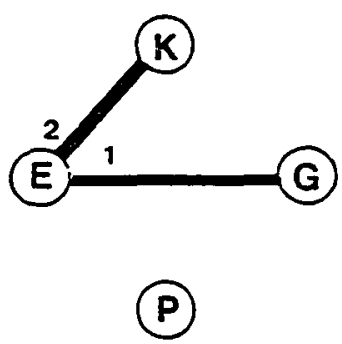

(e)

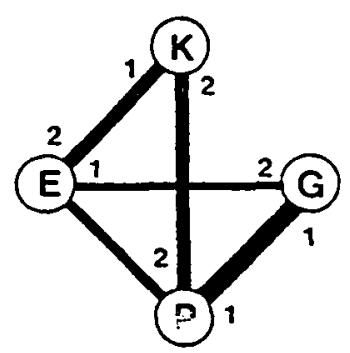

(c)

(6)

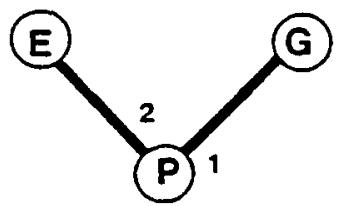

(f)

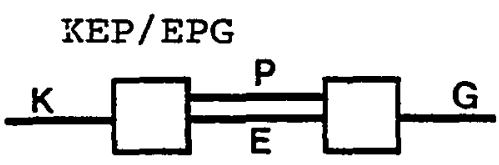

Figure 13. Graphical representation of the dependency analysis for the four-variable system of GENATT, ENRATT, PERCEPT, and KWHUSE. 
The choice of relations to be retained (based on an $80 \%$ threshold) and the final structure are presented in Figure 14 (a) thru (f) and Figure 14 (g) respectively. The structure obtained can be presented in the spectral analysis format. The structure initially has the form of GPEK/EGKP/PGEK/ABK/BA/KEPGA. However, GPER, EGKP, and PGEK are not only identically similar, but, also, embedded in KEPGA. Similarly, BA is embedded in ABK. Therefore, the structure KEPGA/ABK emerges. This structure is presented in Figure 14 (h).

The graph in Figure $14(g)$ is interpreted as variables GENATT, ENRATT, PERCEPT, and KWHUSE being interdependent with varying (but close) strength of relationships. However, the variable AWARE is only related to KWHUSE (significantly) and there is a large amount of interdependence between the variables AWARE and BEHAVE. It is interesting to notice the BEHAVE which was analytically eliminated in the prioritization process (Research Question \#3, Phase II) seems to have little significant direct relationship with the remaining variables. 
TABLE XXI

DEPENDENCY ANALYSIS OF THE SIX-VARIABLE SYSTEM OF SEGMENTATION VARIABLES

\begin{tabular}{|c|c|c|c|c|c|c|}
\hline (I) & (2) & (3) & (4) & (5) & (6) & (7) \\
\hline$i$ & $\mathbf{n}$ & $s_{i}$ & $T\left(i: S_{i}\right)$ & $T_{n} / H(i)$ & $q$ & $T(n)-T(n-I)$ \\
\hline$\overline{\mathbf{G}}$ & $\begin{array}{l}1 \\
2 \\
3 \\
4 \\
5\end{array}$ & $\begin{array}{l}\mathbf{P} \\
\text { PE } \\
\text { PEK } \\
\text { PEKA } \\
\text { PEKAB }\end{array}$ & $\begin{array}{l}0.0434 \\
0.0611 \\
0.0975 \\
0.1550 \\
0.1858\end{array}$ & $\begin{array}{l}0.0279 \\
0.0392 \\
0.0626 \\
0.0995 \\
0.1193\end{array}$ & $\begin{array}{r}23.39 \\
32.86 \\
52.47 \\
83.40 \\
100.00\end{array}$ & $\begin{array}{l}0.0434 \\
0.0177 \\
0.0364 \\
0.0575 \\
0.0308\end{array}$ \\
\hline $\mathbf{E}$ & $\begin{array}{l}1 \\
2 \\
3 \\
4 \\
5\end{array}$ & $\begin{array}{l}\text { G } \\
\text { GK } \\
\text { GKP } \\
\text { GKPA } \\
\text { GKPAB }\end{array}$ & $\begin{array}{l}0.0078 \\
0.0286 \\
0.0695 \\
0.1374 \\
0.1695\end{array}$ & $\begin{array}{l}0.0035 \\
0.0128 \\
0.0310 \\
0.0613 \\
0.0756\end{array}$ & $\begin{array}{r}4.63 \\
16.93 \\
41.01 \\
81.09 \\
100.00\end{array}$ & $\begin{array}{l}0.0078 \\
0.0208 \\
0.0409 \\
0.0679 \\
0.0377\end{array}$ \\
\hline $\mathbf{P}$ & $\begin{array}{l}1 \\
2 \\
3 \\
4 \\
5\end{array}$ & $\begin{array}{l}\text { G } \\
\text { GE } \\
\text { GER } \\
\text { GERA } \\
\text { GERAB }\end{array}$ & $\begin{array}{l}0.0434 \\
0.0586 \\
0.0968 \\
0.1527 \\
0.1863\end{array}$ & $\begin{array}{l}0.0317 \\
0.0428 \\
0.0707 \\
0.1116 \\
0.1361\end{array}$ & $\begin{array}{r}23.29 \\
31.45 \\
51.95 \\
82.00 \\
100.00\end{array}$ & $\begin{array}{l}0.0434 \\
0.0152 \\
0.0382 \\
0.0559 \\
0.0336\end{array}$ \\
\hline $\mathbf{A}$ & $\begin{array}{l}1 \\
2 \\
3 \\
4 \\
5\end{array}$ & $\begin{array}{l}\text { B } \\
\text { BK } \\
\text { BKE } \\
\text { BKEP } \\
\text { BKEPG }\end{array}$ & $\begin{array}{l}0.3935 \\
0.3989 \\
0.4138 \\
0.4442 \\
0.5019\end{array}$ & $\begin{array}{l}0.3884 \\
0.3937 \\
0.4085 \\
0.4385 \\
0.4954\end{array}$ & $\begin{array}{r}78.40 \\
79.47 \\
82.46 \\
88.51 \\
100.00\end{array}$ & $\begin{array}{l}0.3935 \\
0.0054 \\
0.0149 \\
0.0304 \\
0.0577\end{array}$ \\
\hline B & $\begin{array}{l}1 \\
2 \\
3 \\
4 \\
5\end{array}$ & $\begin{array}{l}\text { A } \\
\text { AK } \\
\text { AKE } \\
\text { AKEP } \\
\text { AKEPB }\end{array}$ & $\begin{array}{l}0.3935 \\
0.4004 \\
0.4090 \\
0.4259 \\
0.4567\end{array}$ & $\begin{array}{l}0.6361 \\
0.6473 \\
0.6612 \\
0.6885 \\
0.7383\end{array}$ & $\begin{array}{r}86.16 \\
87.67 \\
89.56 \\
93.26 \\
100.00\end{array}$ & $\begin{array}{l}0.3935 \\
0.0069 \\
0.0086 \\
0.0169 \\
0.0308\end{array}$ \\
\hline $\mathbf{K}$ & $\begin{array}{l}1 \\
2 \\
3 \\
4 \\
5\end{array}$ & $\begin{array}{l}\text { E } \\
\text { EP } \\
\text { EPG } \\
\text { EPGA } \\
\text { EPGAB }\end{array}$ & $\begin{array}{l}0.0076 \\
0.0239 \\
0.0603 \\
0.1220 \\
0.1567\end{array}$ & $\begin{array}{l}0.0053 \\
0.0168 \\
0.0423 \\
0.0856 \\
0.1099\end{array}$ & $\begin{array}{r}4.82 \\
15.29 \\
38.49 \\
77.89 \\
100.00\end{array}$ & $\begin{array}{l}0.0076 \\
0.0163 \\
0.0364 \\
0.0617 \\
0.0347\end{array}$ \\
\hline
\end{tabular}




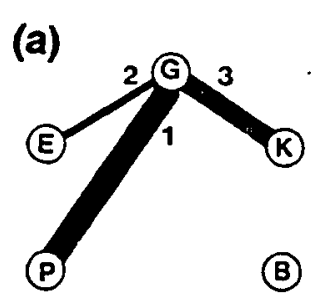

(A)

(d)

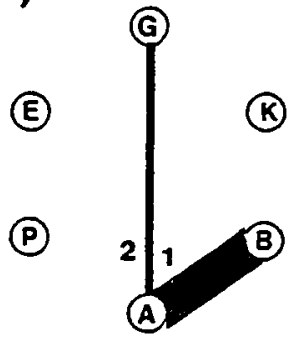

(g)

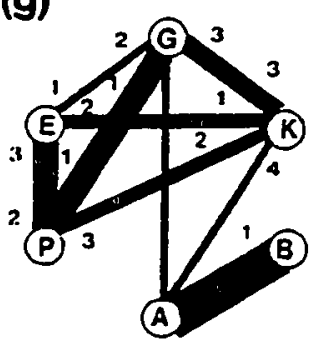

(b)

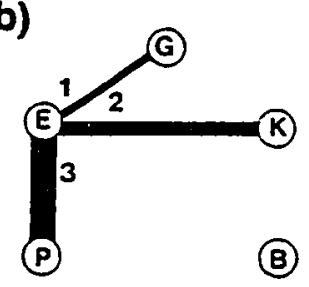

(A) (c)

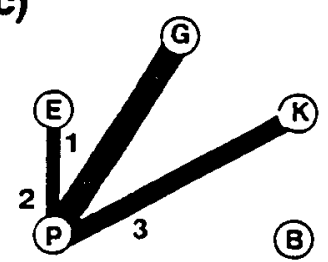

(A)

(f)

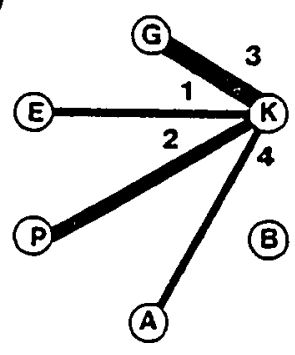

(h) KEPGA/ABK

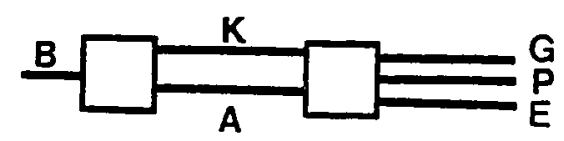

Figure 14. Graphical representation of the dependency structures for the six-variable system of segmentation variables.

Analytical Identification of the Distinguished Segmentation Variable

Research Question \#1. This research question is intended to assess the predictability of each segmentation variable from a set descriptor variables analytically. For the current analyses, each candidate segmentation variable 
is examined for predictability by the other segmentation variables. Table XXII presents the results obtained from the Logit procedure (which is an option within the LOGLINEAR procedure in SPSS ${ }^{x}$ ).

In Table XXII, the logit analysis for each variable, column 1, produces the goodness-of-fit likelihood ratio chisquare, column 2, and its associated degrees of freedom, and risk level, columns 3 and 4, respectively. column 5, 6 and seven, as mentioned earlier, list the measures of entropy, concentration, and the ranking of predictions, respectively. It is evident, based on the ranking, that BEHAVE is the distinguished variable, followed by AWARE, PERCEPT, KWHUSE, and finally, ENRATT.

Again, the information theory model, in table XXI, measures (in column 2) the uncertainty in each variable (as represented by its entropy), the uncertainty of that variable, given the descriptor variables, columns 3 and the amount and the percent of uncertainty removed by the descriptor variables, and the ranking of prediction are given in columns 4 and 5 , respectively. Therefore, the energy related behavior, BEHAVE, is designated as the distinguished variable. In continuing the analyses, therefore, BEHAVE will be treated as the distinguished variable. 
TABLE XXII

PREDICTABIIITY OF CANDIDATES FOR THE

DISTINGUISHED SEGMENTATION VARIABIE

IOG-IINEAR APPROACH (THE IOGIT MODEL)

(1)

Candidate

(i)

ENRATT

PERCEPT

AWARE

BEHAVE

KWHUSE
(2)

(3)

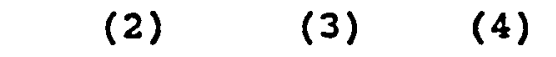
$+$

$I^{2} I I$

df $\mathbf{p}$

$$
683.08
$$

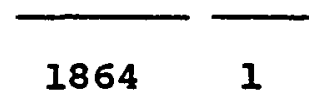

$$
580.05
$$

1755

1

$\begin{array}{lll}445.30 & 1568 & 1\end{array}$

$247.14 \quad 1183 \quad 1$

(5)

Ana. of Disper.

(7)

Entropy Concent Rank
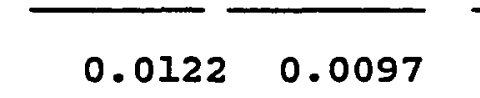

5

$\begin{array}{lll}0.0445 & 0.0371 & 3\end{array}$

$0.4004 \quad 0.3716$

2

$0.6519 \quad 0.6306$

1

$\begin{array}{lll}623.47 & 1864 & 1\end{array}$

$0.0153 \quad 0.0099$

4

\begin{tabular}{|c|c|c|c|c|}
\hline $\begin{array}{l}\text { (1) } \\
\text { Candidate } \\
\text { (i) }\end{array}$ & $\begin{array}{l}\text { (2) } \\
\text { Uncert. of } \\
\text { seg. Var. } \\
H(i)\end{array}$ & $\begin{array}{c}\text { (3) } \\
\text { Uncert of } \\
\text { seg.Var. } \\
\text { given others } \\
\text { H. (i) }\end{array}$ & $\begin{array}{l}(4) \\
\text { Amount ( } 8) \\
\text { Uncert. } \\
\text { Predicted }\end{array}$ & Rank \\
\hline ENRATT & 2.2413 & 2.0663 & $0.1750(7.818)$ & 5 \\
\hline PERCEPT & 1.3687 & 1.1824 & $0.1863(13.618)$ & 3 \\
\hline AWARE & 1.0131 & 0.5111 & $0.5020 \quad(49.55 \%)$ & 2 \\
\hline BEHAVE & 0.6186 & 0.1618 & $0.4568 \quad(73.84 \%)$ & 1 \\
\hline KWHUSE & 1.4256 & 1.2689 & $0.1567(10.998)$ & 4 \\
\hline
\end{tabular}

INFORMATION THEORETIC APPROACH 
Research Question \#2. As the energy related behavior has only two classes, the second research question in Phase II reduces to the results obtained in question \#1 of this phase for the variable BEHAVE. These results are reproduced in Table XXIII.

TABIE XXIII

PREDICTABILITY OF A PARTICUIAR CLASS VS. ALL OTHER CLASSES OF ENERGY RELATED BEHAVIOR

LOG-LINEAR APPROACH (THE LOGIT MODEL)

\begin{tabular}{|c|c|c|c|c|c|}
\hline $\begin{array}{l}\quad \text { (I) } \\
\text { Reclassi- } \\
\text { fication } \\
\text { Scheme for } \\
\text { KWHUSE }\end{array}$ & $I^{2} I I$ & (3) & (4) & $\frac{\text { Ana. of }}{\text { Entropy }}$ & $\frac{\text { Disper. }}{\text { Concent }}$ \\
\hline $\begin{array}{l}\text { Class } 1 \\
\text { Class } 2\end{array}$ & 247.14 & 1183 & 1 & 0.6519 & 0.6306 \\
\hline
\end{tabular}

INFORMATION THEORETIC APPROACH

(1)

Reclassi-

fication

Scheme for

KWHUSE

Class 1

Class 2
(2)

$\frac{\begin{array}{c}\text { Uncert. of } \\ \text { seg. Var. } \\ H(\mathrm{~K})\end{array}}{0.6186}$

(3) Uncert of seg.Var. given others

H. (K)

$\frac{.0}{0.1618}$

(4)

(5)

Amount (q)

Uncert.

Predicted

$0.2568(73.848)$

Descriptions of various columns are given in the previous part, in relation to Table XVIII, which is similar 
to the above table. The high values for the analysis of dispersion (entropy measure $=0.6519$, and concentration $=$ $0.6306)$ for the log-linear model, and the amount of entropy in behave represented by the other variables, indicate that energy related behavior is highly predictable with the knowledge of the other segmentation variables.

Research Question \#3. As expected, both the loglinear approach and the information theoretic approach give the same prioritized set of descriptor variables for BEHAVE. These results are given in Table XXIV. Therefore, variables AWARE, KWHUSE, ENRATT, PRECEPT, GENATT, and BEHAVE form the sequence of priority of contribution to the reduction of uncertainty in BEHAVE.

Research Question \#4. Dependency analysis (similar to other information theoretic structural modeling tools) is a symmetric technique (i.e., it investigates multiple variable simultaneously). The "optimal" dependency structure (subject to the threshold level) for the six-variable system of GENATT, ENRATT, PERCEPT, AWARE, BEHAVE, and KWHUSE will • be the same regardless of which variable is considered as the dependent variable by the investigator. Therefore, the six-variable dependency structure given in connection with KWHUSE as the distinguished variable (see Table XXI and Figure 14), will be the same here, and will not be repeated. 
TABIE XXIV

PRIORITIZATION OF THE EFFECT OF THE OTHER SEGMENTATION VARIABLES ON ENERGY RELATED BEHAVIOR (BEHAVE)

\section{IOG-LINEAR APRBOACF}

Log-Linear Model

(B) (G)

(B) (E)

(B) (P)

(B) (A)

(B) $\{\mathrm{K}\}$

(B) (AG)

(B) $(A E)$

(B) (AP)

(B) (AR)

(B) (ARG)

(B) (AKB)

(B) (AKEG)
(B) (AKEP)

(B) (AKEPG)

\begin{tabular}{|c|c|c|}
\hline$I^{2} I I$ & $d e$ & $\mathbf{p}$ \\
\hline $\begin{array}{r}11.76 \\
16.41 \\
13.09 \\
1819.29 \\
9.91\end{array}$ & $\begin{array}{l}3 \\
4 \\
3 \\
2 \\
4\end{array}$ & $\begin{array}{r}0.008 \\
0.003 \\
0.004 \\
0 \\
0.042\end{array}$ \\
\hline
\end{tabular}

$\begin{array}{lll}1833.95 & 11 & 0 \\ 1834.28 & 14 & 0 \\ 1826.13 & 11 & 0\end{array}$

$1851.30^{\star} 14$

$\begin{array}{lll}1887.27 & 61 & \equiv \\ 1891.07 * & 74 & 0 \\ 1878.44 & 60 & \equiv\end{array}$

1956.46 .299

1969.38 * 299

$2111.75^{\star} 1199$
1

1

INFORMATION THEORETIC APPROACA

\begin{tabular}{|c|c|c|c|c|c|}
\hline Model & $\mathbf{T}$ & $I^{2} I T$ & $d e$ & p & $\cos I^{2} I r$ \\
\hline $\begin{array}{l}T(B: G) \\
T(B: E) \\
T(B: P) \\
T(B: A) \\
T(B: K)\end{array}$ & $\begin{array}{l}0.0025 \\
0.0035 \\
0.0028 \\
0.3935^{\star} \\
0.0021\end{array}$ & $\begin{array}{r}11.55 \\
16.18 \\
12.95 \\
1819.27 \\
9.71\end{array}$ & $\begin{array}{l}3 \\
4 \\
3 \\
2 \\
4\end{array}$ & $\begin{array}{r}\overline{0.008} \\
0.003 \\
0.004 \\
0 \\
0.042\end{array}$ & 1819.27 \\
\hline $\begin{array}{l}T_{A}(B: G) \\
T_{A}(B: E) \\
T_{A}(B: E) \\
T_{A}(B: R)\end{array}$ & $\begin{array}{l}0.0032 \\
0.0032 \\
0.0015 \\
0.0069\end{array}$ & $\begin{array}{r}24.80 \\
14.80 \\
6.94 \\
31.90\end{array}$ & $\begin{array}{r}9 \\
12 \\
9 \\
12\end{array}$ & $\begin{array}{l}0 \\
0 \\
0 \\
0\end{array}$ & 1851.17 \\
\hline $\begin{array}{l}T_{A K}\left(\begin{array}{l}B=G \\
T_{A K}^{A K}\end{array}\right) \\
T_{A K}^{A K}(B: P)\end{array}$ & $\begin{array}{l}0.0078 \\
0.0086 \\
0.0059\end{array}$ & $\begin{array}{l}36.06 \\
39.76 \\
27.28\end{array}$ & $\begin{array}{l}45 \\
60 \\
45\end{array}$ & $\begin{array}{l}\equiv \\
\equiv \\
\vdots \\
0\end{array}$ & 1890.73 \\
\hline $\begin{array}{l}T_{A K E}(B: G) \\
T_{A K E}(B: Q)\end{array}$ & $\begin{array}{l}0.0141 \\
0.0169^{\star}\end{array}$ & $\begin{array}{l}65.19 \\
78.13\end{array}$ & $\begin{array}{l}225 \\
225\end{array}$ & $\frac{1}{1}$ & 1970.06 \\
\hline$T\left(_{\text {AKEP }}(B: G)\right.$ & $0.1018^{\star \prime}$ & 142.40 & 900 & 1 & 2111.46 \\
\hline
\end{tabular}

PRIORITIZATION BASED ON BOTH APPROACHES:

H(BEHAVE) = T(BEHAVE:AWARE) + TAWARE (BETAVE: KWHUSE)

+ TAWARE, KWHUSE (BEFAVE : ENRATT)

- TAWARE, KWHUSE, ENRATT (BEHAVE : PERCERT)

- $T_{\text {AWARE, KWHUSE, ENRATT, ERECEFT (BEHAVE : GENATT) }}$

- HAWARE, KWTUSE, ENRATT, PERCEPT, GENATI ( BEHAVE) 
Next, the three variables in the prioritized set of descriptor variables were selected for structure analysis using conant's method. The variables are AWARE (A), KWHUSE (R), and ENRATT (E) along with the distinguished variable BEHAVE (B) .

This four-variable system (i.e., BEHAVE, AWARE, KWHUSE, ENRATT), however, is different from that analyzed in connection with KWHUSE. This is due to the different orders of prioritization obtained for KWHUSE (as the distinguished variable) versus BEHAVE. 
Dependency Analysis of the Four-Variable System of BEHAVE, AWARE, KWHUSE, AND ENRATT. Table XXV presents the dependency analysis results of the four-variable system of KWHUSE, ENRATT, PERCEPT, and GENATT.

TABLE XXV

DEPENDENCY ANALYSIS OF THE FOUR-VARIABIE SYSTEM OF BEHAVE, AWARE, KWHUSE, AND ENRATT

\begin{tabular}{|c|c|c|c|c|c|c|}
\hline (1) & (2) & (3) & $(4)$ & (5) & (6) & (7) \\
\hline 1 & $\mathbf{n}$ & $s_{i}$ & $T\left(1: s_{i}\right)$ & $T_{n} / H(i)$ & 8 & $T(n)-T(n-1)$ \\
\hline$\overline{\mathbf{B}}$ & $\begin{array}{l}1 \\
2 \\
3\end{array}$ & $\begin{array}{l}\text { A } \\
\text { AKK } \\
\text { AKE }\end{array}$ & $\begin{array}{l}0.3935 \\
0.4004 \\
0.4090\end{array}$ & $\begin{array}{l}0.6361 \\
0.6473 \\
0.6612\end{array}$ & $\begin{array}{r}96.21 \\
97.90 \\
100.00\end{array}$ & $\begin{array}{l}0.39325 \\
0.0069 \\
0.0054\end{array}$ \\
\hline $\mathbf{A}$ & $\begin{array}{l}1 \\
2 \\
3\end{array}$ & $\begin{array}{l}\text { B } \\
\text { BR } \\
\text { BKE }\end{array}$ & $\begin{array}{l}0.3935 \\
0.3989 \\
0.4138\end{array}$ & $\begin{array}{l}0.3884 \\
0.3937 \\
0.4085\end{array}$ & $\begin{array}{r}95.09 \\
96.40 \\
100.00\end{array}$ & $\begin{array}{l}0.3935 \\
0.0054 \\
0.0149\end{array}$ \\
\hline $\mathbf{K}$ & $\begin{array}{l}1 \\
2 \\
3\end{array}$ & $\begin{array}{l}\mathbf{E} \\
\mathbf{E A} \\
E A B\end{array}$ & $\begin{array}{l}0.0076 \\
0.0159 \\
0.0282\end{array}$ & $\begin{array}{l}0.0053 \\
0.0112 \\
0.0198\end{array}$ & $\begin{array}{r}26.95 \\
56.38 \\
100.00\end{array}$ & $\begin{array}{l}0.0016 \\
0.0015 \\
0.0021\end{array}$ \\
\hline $\mathbf{E}$ & $\begin{array}{l}1 \\
2 \\
3\end{array}$ & $\begin{array}{l}\mathrm{K} \\
\mathrm{KA} \\
\mathrm{KAB}\end{array}$ & $\begin{array}{l}0.0076 \\
0.0205 \\
0.0291\end{array}$ & $\begin{array}{l}0.0034 \\
0.0092 \\
0.0130\end{array}$ & $\begin{array}{r}26.12 \\
70.45 \\
100.00\end{array}$ & $\begin{array}{l}0.0076 \\
0.0129 \\
0.0086\end{array}$ \\
\hline
\end{tabular}

Using the threshold level of $80 \%$, the dependency structures with respect to each variable are given in Figure 15 (a thru d). The total dependency structure is presented in Figure 15 (e). The structure in Figure 15 (e) is shown in it alternative format in Figure 15 (f). 
(a)

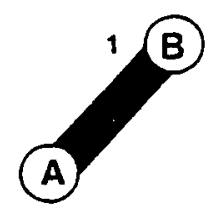

()
(E)

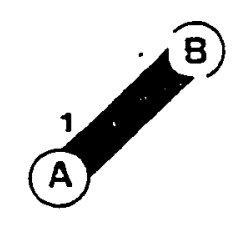

(K) (c)

(B)

(E)

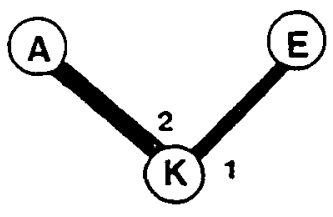

(f)

(e)

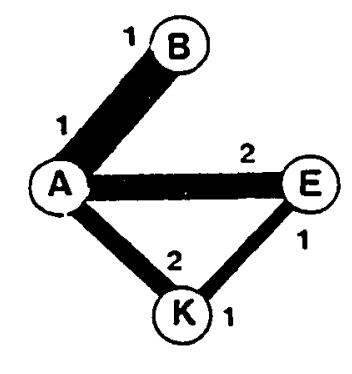

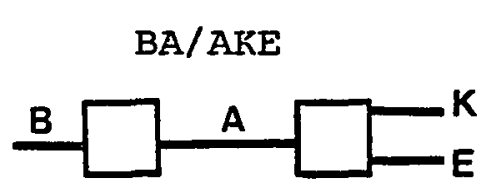

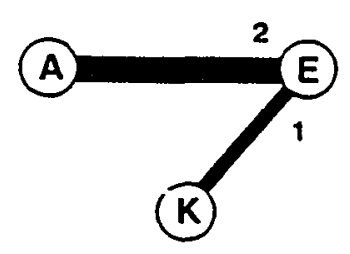

(B)

Fiqure 15. Graphical representation for the dependency structures for the four-variable system of BEHAVE, AWARE, KWHUSE, and ENRATT.

After removing the embedded and identical subsystems. from $\mathrm{BA} / \mathrm{AKE} / \mathrm{KEA} / \mathrm{EAK}$, the structure BA/AKE (Figure 15 (f)) emerges. This structure can be interpreted as follows. Energy related behavior, BEHAVE, directly interact with energy related awareness, AWARE, which has a direct effect on electricity consumption, KWHUSE, and energy related 
attitudes, ENRATT. The latter three variables have mutual effect on each other.

\section{DETERMINATION AND ANALYSIS OF THE DISTIN- GUISHED SEGMENTATION VARIABLE USING EXOGENOUS DESCRIPTOR VARIABLES}

The previous section conducted Phase II of segment congruence analysis using endogenous variables (i.e., other segmentation variables), as descriptor variable, to identify the distinguished segmentation variable and analyze it through structure analysis techniques. This section introduces a set of exogenous variables (or clusterings) to be used as descriptor variables. Then the analysis in Phase II will be repeated using these new descriptor variables.

Table XI in chapter III presents these descriptor variables, and Table $x$ lists the variables selected from the PNWRES data (see Appendix IV for a complete list of variables). Also, in Chapter III, the procedures used to obtain and cluster all of the variables used in this study are described. Table XXVI reproduces the listing of the exogenous variables for ease of reference.

The analysis will proceed with the evaluation of the candidate segmentation variables, obtained in section 5.1 . through the above descriptor variables. These candidate variables are ENRATT, PERCEPT, AWARE, BEHAVE, and KWHUSE. 
TABLE XXVI

IIST OF THE EXOGENOUS DESCRIPTOR VARIABLES

\begin{tabular}{lllll}
\hline $\begin{array}{l}\text { Variable } \\
\text { (Abbreviation) }\end{array}$ & Description & & \# of classes \\
CLIMGEO & (C) & Climatic/Geographic Environment & 3 \\
TYPDWEL & (T) & Type of the Dwelling Unit & & 4 \\
RENTOWN & (R) & The Dwelling is Rental/Owned & 3 \\
DEMOG & (D) & Demographic Characteristics & 3 \\
INSUL & (I) & Level of Insulation & 3 \\
\hline
\end{tabular}

Research question \#I. Table XXVII shows the results of five logit models, each using one of the candidates for the distinguished variable as the dependent variable against the exogenous descriptor variables. The information theoretic analysis, also presented in Table XXVII, calculates the amount of uncertainty in each candidate variable reduced by the descriptor variables.

It is seen that both methods result in the same distinguished variable (i.e., KWHUSE). The two methods, as expected, give identical ranking of the candidates for the distinguished variable, as well. 
TABLE XXVII

PREDICTABIIITY OF CANDIDATES FOR THE

DISTINGUISHED SEGMENTATION VARIABLE USING

EXOGENOUS DESCRIPTOR VARIABLES

\section{IOG-IINEAR APPROACH (THE LOGIT MODEL)}

\begin{tabular}{|c|c|c|c|c|c|c|}
\hline $\begin{array}{c}\text { (1) } \\
\text { Candidate } \\
\text { (i) }\end{array}$ & $\begin{array}{l}(2) \\
I^{2} I I\end{array}$ & $\begin{array}{l}(3) \\
\text { df }\end{array}$ & (4) & $\begin{array}{l}\text { (5) } \\
\text { Ana. of } \\
\text { Entropy }\end{array}$ & $\begin{array}{c}(6) \\
\text { Disper. } \\
\text { Concent }\end{array}$ & $\begin{array}{r}(7) \\
\text { Rank }\end{array}$ \\
\hline ENRATT & 627.89 & 1680 & 1 & 0.0209 & 0.0171 & 4 \\
\hline PERCEPT & 400.12 & 1260 & 1 & 0.0110 & 0.0103 & 5 \\
\hline AWARE & 330.90 & 840 & 1 & 0.0336 & 0.0358 & 3 \\
\hline BEHAVE & 163.81 & 420 & 1 & 0.0504 & 0.0482 & 2 \\
\hline KWHUSE & 408.95 & 1640 & 1 & 0.0632 & 0.0392 & 1 \\
\hline
\end{tabular}

INFORMATION THEORETIC APPROACH

(1)

Candidate

(i)

ENRATT

PERCEPT

AWARE

BEHAVE

KWHUSE
(2) Uncert. of seg. Var. H(i)

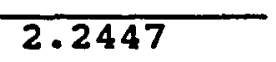

1.3581

1.0354

0.6436

1.4171
(3)

Uncert of

seg.Var.

given others

H. (i)

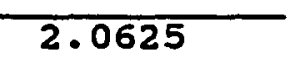

1.2486

0.9284

0.5760

1.2304
(4)

Amount ( 8 )

Uncert.

Predicted Rank

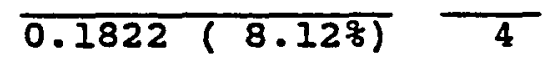

$0.1095(8.06 \%) \quad 5$

$0.1070(10.33 \%) \quad 3$

$0.0676(10.50 \%) \quad 2$

$0.1867(13.18 \%) \quad 1$

Having identified the distinguished segmentation variable KWHUSE, the remaining analyses concentrate on 
assessing the relationships and the structure of the system composed of this variable and the descriptor variables. First, the question of predictability will be pursued further in question \#2. Next, the descriptor variables will be prioritized in question \#3. Finally, the structure of relationships will be assessed in questions \#4 and \#5.

Research Question \#2. The distinguished variable (KWHUSE) was reclassified based on three collapsing schemes (i.e., the same three groupings as used in section 5.2). First, the low-consumption group was aggregated (i.e., classes 1 and 2 ) as class \#1, and the remaining classes were aggregated (i.e., classes 3,4 , and 5) as class \#2. Then, the medium-consumption class (i.e., class \#3) was dichotomized against all other classes combined. Finally, the high-consumption group (i.e., classes 4 and 5) were dichotomized against all other classes.

Then, both the log-Iinear approach (i.e., logit models) and the information theoretic approach were used to assess the differences in the three grouping schemes. The results are presented in Table XXVIII.

It is evident that the high-consumption/other-groups scheme has the highest predictability measures followed by the low-consumption/others, and medium-consumption/others, as second and third best represented schemes, respectively. 
TABLE XXVIII

PREDICTABILITY OF A PARTICULAR CLASS VS. ALI OTHER CLASSES OF THE DISTINGUISHED VARIABLE, (KWHUSE)

\section{IOG-IINEAR APPROACH}

(1)

Reclassi-

fication

Scheme for

KWHUSE

Class $1 \& 2=1$
Others $=2$

(2)

(3)

(4)

(5)

(6)

(7)

Class $3=1$

others $=2$

\begin{tabular}{|c|c|c|c|c|}
\hline$I^{2} I I$ & $d f$ & $\mathbf{p}$ & $\frac{\text { Ana. of }}{\text { Entropy }}$ & $\frac{\text { Disper. }}{\text { Concent }}$ \\
\hline 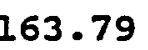 & 420 & 1 & 0.0466 & 0.0560 \\
\hline
\end{tabular}

Class $4 \& 5=1$

Others $=2$

193.55

420

$1 \quad 0.0176$

0.0235

145.07

420

$1 \quad 0.0945$

0.0717

\section{INFORMATION THEORETIC APPROACH}

(1)

Reclassi-

fication

Scheme for

KWHUSE

Class 1\&2 $=1$

others $=2$

Class $3=1$

Others $=2$

Class $4 \& 5=1$
(2)

(3) Uncert of Uncert. of seg.Var. seg. Var given others Amount (\%) H(II) Uncert.

H. (K) Predicted

(4)

(5)

Others $=2$

0.4848

0.4045

16.56 
This, basically, indicates that given the descriptor variables, the uncertainty with respect to highconsumption/others is less than other groupings.

Research Question \#3. Table XXIX presents the logIinear approach and the information theoretic approach to prioritization of contributions of the descriptor variables to the distinguished segmentation variable, KwHUSE. At the bottom of the table, this prioritization is expressed in information theoretic terms.

Here, the descriptor variables DEMOG, TYPDWEL, CIIMGEO, INSUL, and RENTOWN contribute to the representation of distinguished variable. Their priority is in the sequence given above. Therefore, the three most significant descriptor variables needed for research question \#4 are DEMOG, TYPDWEL, and CLIMGEO. 
TABLE XXIX

PRIORITIZED EFFECT OF THE EXOGENOUS DESCRIPTOR VARIABLES ON ENERGY CONSUMPTION

IOG-IMNAAR APPROACE

Iog-Linear ModeI

(R)(c)

\{K\} (T)

(R) (R)

(K) $(\mathrm{D})$

(R) (DC)

(K) (DT)

(R) (DI)

$\{$ R $\}$ (DTC)

(R) (DTI)

(R) (DTCR)

(R) $\{E P G B\}$

(R) (EPGAB)

\begin{tabular}{|c|c|c|}
\hline$I^{2} I I$ & $d e$ & p \\
\hline $\begin{array}{r}56.87 \\
175.22 \\
46.78 \\
182.35 \\
28.75\end{array}$ & $\begin{array}{r}8 \\
12 \\
8 \\
12 \\
8\end{array}$ & $\begin{array}{r}= \\
0 \\
0 \\
0 \\
0\end{array}$ \\
\hline $\begin{array}{l}260.81 \\
313.10 \\
247.06 \\
217.56\end{array}$ & $\begin{array}{l}44 \\
63 \\
44 \\
44\end{array}$ & $\begin{array}{l}0 \\
\vdots \\
0 \\
0 \\
0\end{array}$ \\
\hline $\begin{array}{l}442.06^{*} \\
399.17 \\
386.90\end{array}$ & $\begin{array}{l}211 \\
211 \\
210\end{array}$ & $\equiv \begin{array}{l}0 \\
\vdots \\
0\end{array}$ \\
\hline $\begin{array}{l}565.32 \\
587.93\end{array}$ & $\begin{array}{l}595 \\
595\end{array}$ & $\begin{array}{l}0 \\
0\end{array}$ \\
\hline 786.05 & 1747 & 0 \\
\hline
\end{tabular}

IWEORYATION THEORETIC APPROACH

\begin{tabular}{|c|c|c|c|c|c|}
\hline Model & $\mathbf{T}$ & $I^{2} I T$ & de & P & $\cos I^{2} I T$ \\
\hline $\begin{array}{l}T(R: C) \\
T(R: T) \\
T(R: R) \\
T(R: D) \\
T(K: I)\end{array}$ & $\begin{array}{l}0.0135 \\
0.0416 \\
0.0111 \\
0.0433 \\
0.0068\end{array}$ & $\begin{array}{r}56.84 \\
175.14 \\
46.73 \\
182.30 \\
28.63\end{array}$ & $\begin{array}{r}8 \\
12 \\
8 \\
12 \\
8\end{array}$ & $\begin{aligned} &= 0 \\
& 0 \\
& 0 \\
& 0 \\
& 0\end{aligned}$ & 182.30 \\
\hline $\begin{array}{l}T_{D}(K: C) \\
T_{D}^{(K: T} \\
T_{D}(K: R) \\
T_{D}(K: I)\end{array}$ & $\begin{array}{l}0.0186 \\
0.0311 \\
0.0154 \\
0.0080\end{array}$ & $\begin{array}{r}78.31 \\
130.94 \\
64.84 \\
33.68\end{array}$ & $\begin{array}{l}32 \\
51 \\
32 \\
32\end{array}$ & $\begin{array}{l}\equiv 0 \\
\equiv 0 \\
\vdots \\
0 \\
0\end{array}$ & 313.24 \\
\hline $\begin{array}{l}T_{D T}(K: C) \\
T_{D T}(R: R) \\
T_{D T}(K: I)\end{array}$ & $\begin{array}{l}0.0286^{\star} \\
0.0168 \\
0.0150\end{array}$ & $\begin{array}{r}120.41 \\
70.73 \\
63.25\end{array}$ & $\begin{array}{l}148 \\
148 \\
148\end{array}$ & $\begin{array}{l}\equiv 0 \\
\equiv 0 \\
=0\end{array}$ & 433.65 \\
\hline $\begin{array}{l}T_{\text {DTC }}(\mathrm{X}: R) \\
T_{\text {DTC }}(\mathrm{K}: I)\end{array}$ & $\begin{array}{l}0.0314 . \\
0.0367\end{array}$ & $\begin{array}{l}132.20 \\
154.51\end{array}$ & $\begin{array}{l}384 \\
384\end{array}$ & $\begin{array}{l}0 \\
0\end{array}$ & 588.16 \\
\hline$T($ DTCI $(K: R)$ & $0.0471^{\star}$ & 198.30 & 1152 & 0 & 786.46 \\
\hline
\end{tabular}

PRIORITIZATION BASED ON BOTH APPROACHES:

H(KWHUSE) $=T$ (KWHUSE:DEMOG) - TDEMOG (KWHUSE:TYPDWEI)

+ TDEMOG, TYPDWEL (KWHUSE: CLIMGEO)

+ TDEMOG, TYPDWEL, CLIMGEO (KWHUSE : INSUL)

- TDEMOG, TYPDWEI, CLIMGEO, INSUI (KWHUSE: RENTOWN)

+ HDEMOG, TYPDWEL, CLIMGEO, INSUL, RENTOWN (KWHUSE) 
Research Question \#4. Dependency Analysis of the Four-

Variable System of KWHUSE, DEMOG, TYPDWEL, and CLIMGEO. The results of dependency analysis of KWHUSE, DEMOG, TYPDWEL, and CIIMgEO are given in Table XXX.

TABLE XXX

DEPENDENCY ANALYSIS OF THE FOUR-VARIABLE SYSTEM OF KWHUSE, DEMOG, TYPDWEL, AND CLIMGEO

\begin{tabular}{|c|c|c|c|c|c|c|}
\hline (1) & (2) & (3) & (4) & (5) & (6) & (7) \\
\hline $\mathbf{i}$ & $\mathbf{n}$ & $s_{i}$ & $T\left(i: s_{i}\right)$ & $T_{n} / H(i)$ & 8 & $T(n)-T(n-1)$ \\
\hline $\mathbf{R}$ & $\begin{array}{l}1 \\
2 \\
3\end{array}$ & $\begin{array}{l}\text { D } \\
\text { DT } \\
\text { DTC }\end{array}$ & $\begin{array}{l}0.0433 \\
0.0744 \\
0.1030\end{array}$ & $\begin{array}{l}0.0306 \\
0.0525 \\
0.0727\end{array}$ & $\begin{array}{r}42.04 \\
72.23 \\
100.00\end{array}$ & $\begin{array}{l}0.0433 \\
0.0311 \\
0.0286\end{array}$ \\
\hline D & $\begin{array}{l}1 \\
2 \\
3\end{array}$ & $\begin{array}{l}\text { T } \\
\text { TK } \\
\text { TKC }\end{array}$ & $\begin{array}{l}0.0762 \\
0.1090 \\
0.1301\end{array}$ & $\begin{array}{l}0.0387 \\
0.0553 \\
0.0661\end{array}$ & $\begin{array}{r}58.57 \\
83.78 \\
100.00\end{array}$ & $\begin{array}{l}0.0762 \\
0.0328 \\
0.0211\end{array}$ \\
\hline $\mathbf{T}$ & $\begin{array}{l}1 \\
2 \\
3\end{array}$ & $\begin{array}{l}\text { D } \\
\text { DR } \\
\text { DRC }\end{array}$ & $\begin{array}{l}0.0762 \\
0.1073 \\
0.1222\end{array}$ & $\begin{array}{l}0.0835 \\
0.1175 \\
0.1339\end{array}$ & $\begin{array}{r}62.36 \\
87.81 \\
100.00\end{array}$ & $\begin{array}{l}0.0762 \\
0.0311 \\
0.0149\end{array}$ \\
\hline C & $\begin{array}{l}1 \\
2 \\
3\end{array}$ & $\begin{array}{l}\mathrm{R} \\
\mathrm{KD} \\
\mathrm{KDT}\end{array}$ & $\begin{array}{l}0.0135 \\
0.0261 \\
0.0410\end{array}$ & $\begin{array}{l}0.0085 \\
0.0165 \\
0.0259\end{array}$ & $\begin{array}{r}32.93 \\
63.66 \\
100.00\end{array}$ & $\begin{array}{l}0.0135 \\
0.0126 \\
0.0149\end{array}$ \\
\hline
\end{tabular}

Again, assuming $80 \%$ threshold, the graphs of dependencies were obtained for each variable. These graphs along with the total graph are given in Figure 16 (a thru e). Also, the alternative representation of the total graph is given in Figure 16 (f). 
(a)

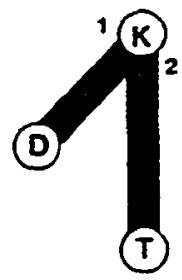

(C)

(d)

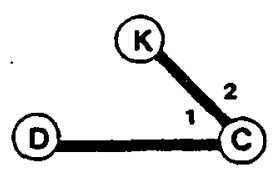

(T) (b)

(

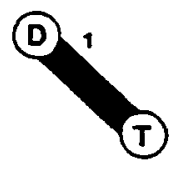

(C)

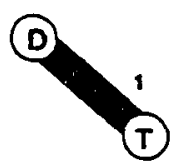

(C)

(c)

(K)

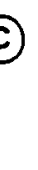

(e)

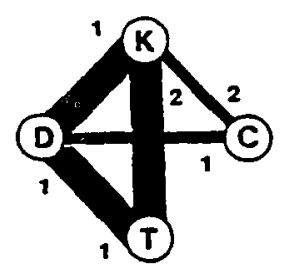

Figure 16. Graphical representation of the dependency structures for the four-variable system of KWHUSE, DEMOG, TYPDWEL, and CLIMGEO.

The emerging structural model based on conant's dependency. analysis is $\mathrm{KDC} / \mathrm{KDT}$. This structure implies that electricity consumption, KWHUSE, is directly affected by the type of dwelling, TYPDWEL, and the geoclimatic environment, CLIMGEO. The demographic variable has direct relationships with KWHUSE, ans CLIMGEO on the one hand, and with KWHUSE and TYPDWEL on the other.

Dependency Analysis of the Six-Variable System of KWHUSE, ENRATT, PERCEPT, GENATT, AWARE, and BEHAVE. Table XXXI shows the dependency analysis results. 
TABIE XXXI

DEPENDENCY ANALYSIS OF THE DISTINGUISHED

VARIABLE AND THE EXOGENOUS DESCRIPTOR

VARIABLES

\begin{tabular}{|c|c|c|c|c|c|c|}
\hline (I) & $(2)$ & (3) & (4) & (5) & (6) & (7) \\
\hline$i$ & $\mathbf{n}$ & $s_{i}$ & $T\left(i: S_{i}\right)$ & $T_{n} / H(i)$ & 8 & $T(n)-T(n-1)$ \\
\hline$\overline{\mathbf{K}}$ & $\begin{array}{l}1 \\
2 \\
3 \\
4 \\
5\end{array}$ & $\begin{array}{l}\text { D } \\
\text { DI } \\
\text { DTC } \\
\text { DTCI } \\
\text { DTCIR }\end{array}$ & $\begin{array}{l}0.0433 \\
0.0744 \\
0.1030 \\
0.1397 \\
0.1868\end{array}$ & $\begin{array}{l}0.0306 \\
0.0525 \\
0.0727 \\
0.0986 \\
0.1318\end{array}$ & $\begin{array}{r}23.22 \\
39.83 \\
55.16 \\
74.81 \\
100.00\end{array}$ & $\begin{array}{l}0.0433 \\
0.0311 \\
0.0286 \\
0.0367 \\
0.0471\end{array}$ \\
\hline C & $\begin{array}{l}1 \\
2 \\
3 \\
4 \\
5\end{array}$ & $\begin{array}{l}\mathrm{K} \\
\mathrm{KD} \\
\mathrm{KDI} \\
\mathrm{KDIT} \\
\mathrm{KDITR}\end{array}$ & $\begin{array}{l}0.0135 \\
0.0261 \\
0.0438 \\
0.0745 \\
0.1151\end{array}$ & $\begin{array}{l}0.0085 \\
0.0165 \\
0.0277 \\
0.0470 \\
0.0727\end{array}$ & $\begin{array}{r}11.69 \\
22.70 \\
38.10 \\
64.65 \\
100.00\end{array}$ & $\begin{array}{l}0.0135 \\
0.0126 \\
0.0177 \\
0.0307 \\
0.0406\end{array}$ \\
\hline $\mathbf{T}$ & $\begin{array}{l}1 \\
2 \\
3 \\
4 \\
5\end{array}$ & $\begin{array}{l}\text { D } \\
\text { DK } \\
\text { DKC } \\
\text { DKCI } \\
\text { DKCIR }\end{array}$ & $\begin{array}{l}0.0762 \\
0.1073 \\
0.1492 \\
0.1753 \\
0.2076\end{array}$ & $\begin{array}{l}0.0835 \\
0.1175 \\
0.1634 \\
0.1920 \\
0.2274\end{array}$ & $\begin{array}{r}36.72 \\
51.67 \\
71.86 \\
84.43 \\
100.00\end{array}$ & $\begin{array}{l}0.0762 \\
0.0311 \\
0.0419 \\
0.0261 \\
0.0323\end{array}$ \\
\hline $\mathbf{R}$ & $\begin{array}{l}1 \\
2 \\
3 \\
4 \\
5\end{array}$ & $\begin{array}{l}\text { D } \\
\text { DI } \\
\text { DIR } \\
\text { DIKC } \\
\text { DIKCT }\end{array}$ & $\begin{array}{l}0.0217 \\
0.0371 \\
0.0628 \\
0.0930 \\
0.1253\end{array}$ & $\begin{array}{l}0.0321 \\
0.0549 \\
0.0929 \\
0.1375 \\
0.1853\end{array}$ & $\begin{array}{r}17.32 \\
29.63 \\
50.14 \\
74.20 \\
100.00\end{array}$ & $\begin{array}{l}0.0217 \\
0.0154 \\
0.0257 \\
0.0302 \\
0.0323\end{array}$ \\
\hline D & $\begin{array}{l}1 \\
2 \\
3 \\
4 \\
5\end{array}$ & $\begin{array}{l}\text { T } \\
\text { TK } \\
\text { TKR } \\
\text { TKRC } \\
\text { TKRCI }\end{array}$ & $\begin{array}{l}0.0762 \\
0.1090 \\
0.1328 \\
0.1670 \\
0.2208\end{array}$ & $\begin{array}{l}0.0387 \\
0.0553 \\
0.0674 \\
0.0848 \\
0.1121\end{array}$ & $\begin{array}{r}34.52 \\
49.33 \\
60.13 \\
75.65 \\
100.00\end{array}$ & $\begin{array}{l}0.0762 \\
0.0328 \\
0.0238 \\
0.0342 \\
0.0538\end{array}$ \\
\hline I & $\begin{array}{l}1 \\
2 \\
3 \\
4 \\
5\end{array}$ & $\begin{array}{l}\mathbf{R} \\
\text { RD } \\
\text { RDK } \\
\text { RDKC } \\
\text { RDKCT }\end{array}$ & $\begin{array}{l}0.0148 \\
0.0209 \\
0.0396 \\
0.0715 \\
0.1075\end{array}$ & $\begin{array}{l}0.0123 \\
0.0174 \\
0.0330 \\
0.0596 \\
0.0896\end{array}$ & $\begin{array}{r}13.73 \\
19.42 \\
36.83 \\
66.52 \\
100.00\end{array}$ & $\begin{array}{l}0.0148 \\
0.0061 \\
0.0187 \\
0.0319 \\
0.0360\end{array}$ \\
\hline
\end{tabular}


The choice of relations to be retained (based on an $80 \%$ threshold) and the final structure are presented in Figure 17 (a) thru (f) and Figure 17 (g) respectively.

(a)

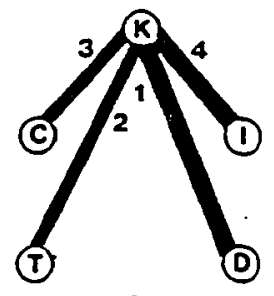

(B)

(d)

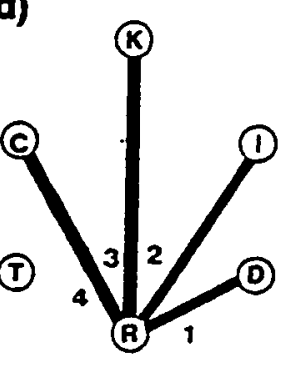

(g)

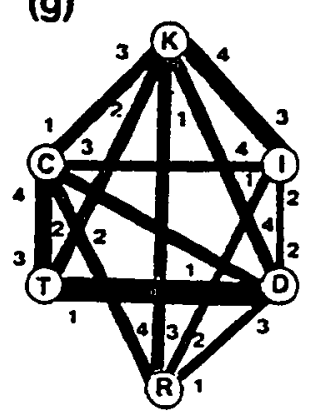

(b)

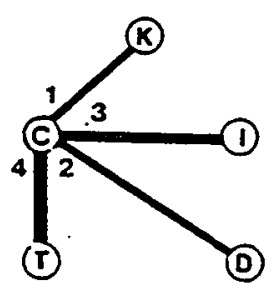

(B)

(e)

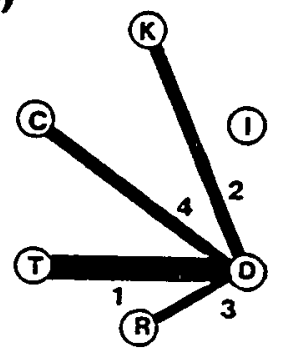

(c)

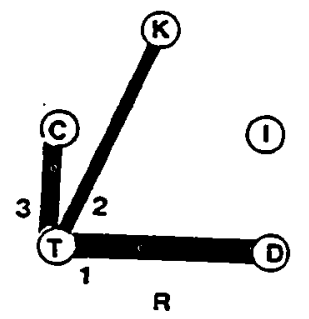

(f)

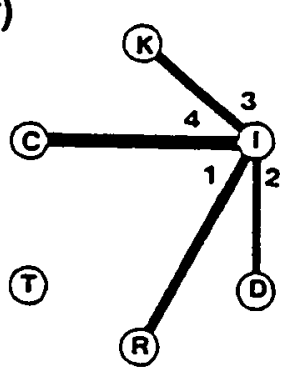

Figure 17. Graphical representation of the dependency structures for the system of distinguished and exogenous variables.

(h) $\mathrm{KCTDI} / \mathrm{KCRDI} / \mathrm{KCTRD}$

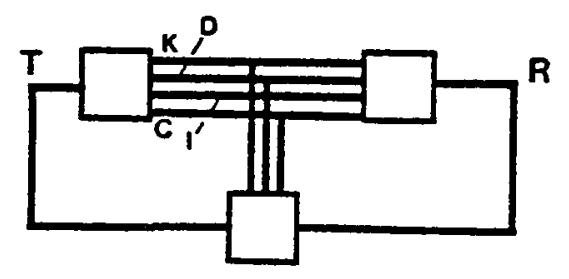


The dependency structure in Figure 17 (g) can be represented as KDTCI/CKDIT/TDKC/RDIKC/DTKRC/IRDKC. However, the first and the second terms in the above structure are identical. Also, TDKC (the third term) is embedded in the first two terms. Furthermore, the fourth and the sixth terms are identical. Therefore, the structure is rewritten, eliminating all but one of the identical terms, as RCTDI/RCRDI/RCTRD. This structure is given in Figure 17 (h) .

\section{SUMMARY OF ANALYSIS}

This chapter used the PNWRES data to demonstrate that all of the proposed questions in segment congruence analysis can be addressed through the proposed methodology. The equivalent log-linear models were proposed to perform these analyses, where applicable and practical. Tables XXXII through XXXV present all of the results obtained throughout this chapter in a brief format.

It is clear that most of the proposed analyses can be performed using either method. However, all of the results were obtained through three main SYSENT runs plus some additional minor runs. The log-linear approach on the other hand required hundreds of runs. Additionally, the amounts of information and/or uncertainty contained in each model could not be obtained through the log-linear approach. 
Therefore, it is proposed that the researchers use the information theoretic approach for exploratory analyses and identification of important variables. After these variables are identified, the algorithms common in loglinear computer packages (e.g., the proportional fitting method) can be used to obtain cell estimates and predictions in a disaggregate form.

TABLE XXXII

ANALYSIS OF THE MUTUAL ASSOCIATION AMONG SEGMENTATION VARIABLES AND IDENTIFICATION

OF CANDIDATES FOR THE DISTINGUISHED SEGMENTATION VARIABLE

\begin{tabular}{|c|c|c|}
\hline Description & $\begin{array}{l}\text { Information } \\
\text { Theoretic } \\
\text { Approach }\end{array}$ & $\begin{array}{l}\text {-Iinear/ } \\
\text { Logit } \\
\text { pproach }\end{array}$ \\
\hline $\begin{array}{l}\text { A) MUTUAL ASSOCIATION } \\
\text { Existence } \\
\text { Amount }\end{array}$ & $\begin{array}{c}\text { Yes } \\
T / T_{\text {MAX }}=0.0765\end{array}$ & Yes \\
\hline \multicolumn{3}{|l|}{$\begin{array}{l}\text { B) CANDIDATES FOR THE } \\
\text { DISTINGUISHED BASE }\end{array}$} \\
\hline $\begin{array}{l}\text { Contribution to } \\
\text { Mutual Association }\end{array}$ & AWARE & AWARE \\
\hline $\begin{array}{l}\text { Contribution to } \\
\text { Joint Variability }\end{array}$ & $\begin{array}{l}\text { (aware) } \\
\text { BEHAVE }\end{array}$ & $\begin{array}{l}\text { (aware) } \\
\text { BEHAVE }\end{array}$ \\
\hline $\begin{array}{l}\text { Contribution to } \\
\text { Uncertainty of System }\end{array}$ & ENRATT, (aware), (behave) & $\sim$ \\
\hline $\begin{array}{l}\text { Number of Significant } \\
\text { Dyadic Relations }\end{array}$ & PERCEPT & PERCEPT \\
\hline JUDGMENT-BASED CANDIDATE & KWHUSE & KWHUSE \\
\hline
\end{tabular}




\section{TABIE XXXIII}

\section{ANALYSIS USING OTHER SEGMENTATION VARIABLES AS DESCRIPTOR VARIABLES}

ELECTRICITY CONSUMPTION AS THE DISTINGUISHED VARIABLE (스 PRIORI)

\begin{tabular}{|c|c|c|}
\hline Description & $\begin{array}{l}\text { Information } \\
\text { Theoretic } \\
\text { Approach }\end{array}$ & $\begin{array}{c}\text { Log-Iinear/ } \\
\text { Logit } \\
\text { Approach }\end{array}$ \\
\hline $\begin{array}{l}\text { PREDICTABIIITY OF COMBINED } \\
\text { CLASSES }\end{array}$ & \multicolumn{2}{|c|}{ (Ranking) } \\
\hline $\begin{array}{l}\text { Class } 1 \& 2 / \text { Others (Low) } \\
\text { Class } 3 / \text { Others (Medium) } \\
\text { Class } 4 \& 5 / \text { Others (High) }\end{array}$ & $\begin{array}{l}(2) \\
(3) \\
(1)\end{array}$ & $\begin{array}{l}(2) \\
(3) \\
(1)\end{array}$ \\
\hline $\begin{array}{l}\text { PRIORITIZATION OF DESCRIPTOR } \\
\text { VARIABIES }\end{array}$ & \multicolumn{2}{|c|}{ (Priority) } \\
\hline $\begin{array}{l}\text { GENATT } \\
\text { ENRATT } \\
\text { PERCEPT } \\
\text { AWARE } \\
\text { BEHAVE }\end{array}$ & $\begin{array}{l}(3) \\
(1) \\
(2) \\
(4) \\
(5)\end{array}$ & $\begin{array}{l}(3) \\
(1) \\
(2) \\
(4) \\
(5)\end{array}$ \\
\hline $\begin{array}{l}\text { STRUCTURE ANALYSIS } \\
\text { The 4-variable system }\end{array}$ & EPK/PG & EPK/PG \\
\hline The 6-variable system & KEPGA/ABK & KEPGA/ABK \\
\hline
\end{tabular}


TABLE XXXIV

\section{ANALYSIS USING OTHER SEGMENTATION VARIABLES \\ AS DESCRIPTOR VARIABLES}

ANALYTICAL IDENTIFICATION AND ANALYSIS OF THE DISTINGUISHED VARIABIE

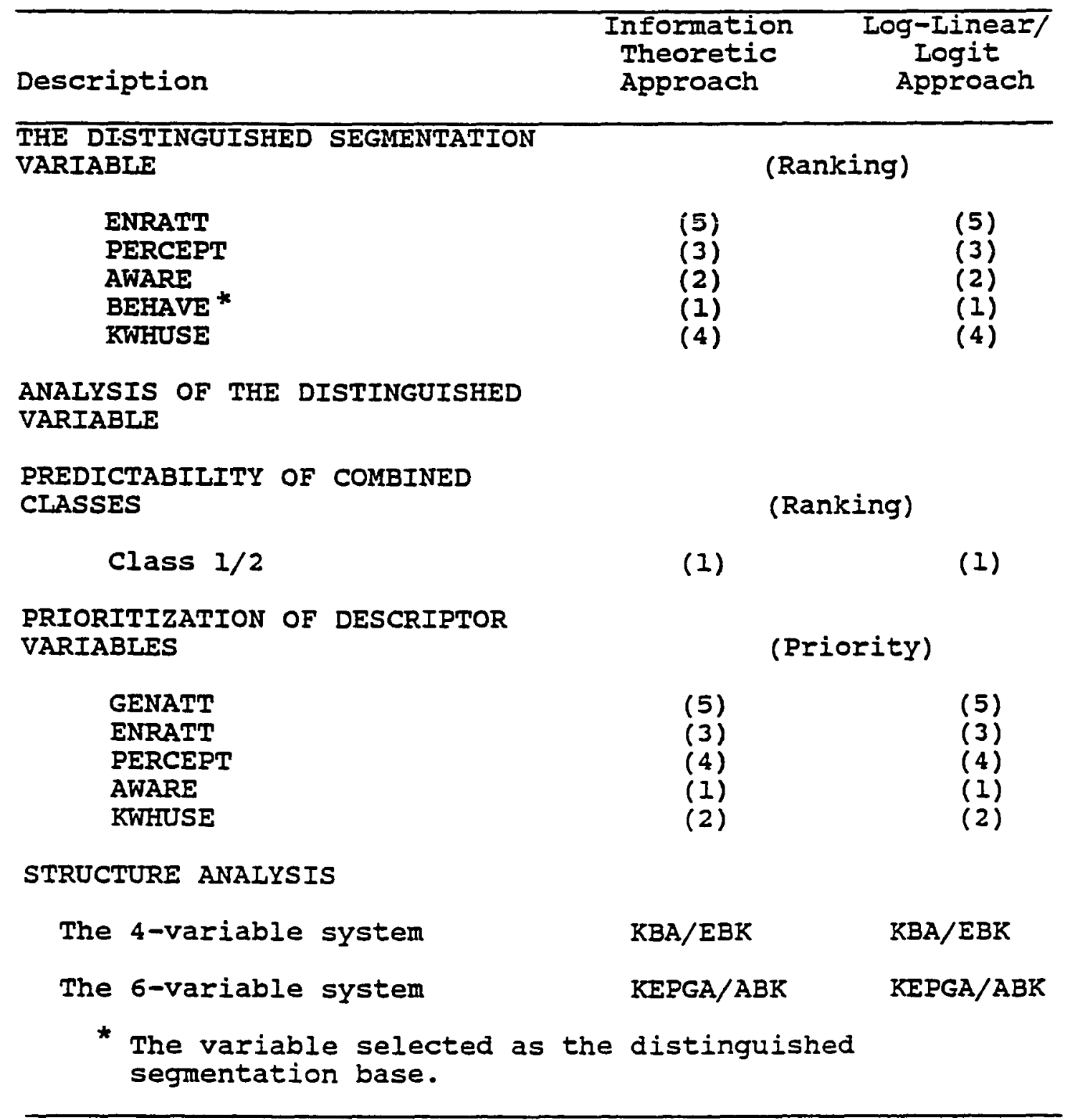


TABLE XXXV

\section{ANALYSIS USING EXOGENOUS DESCRIPTOR} VARIABLES

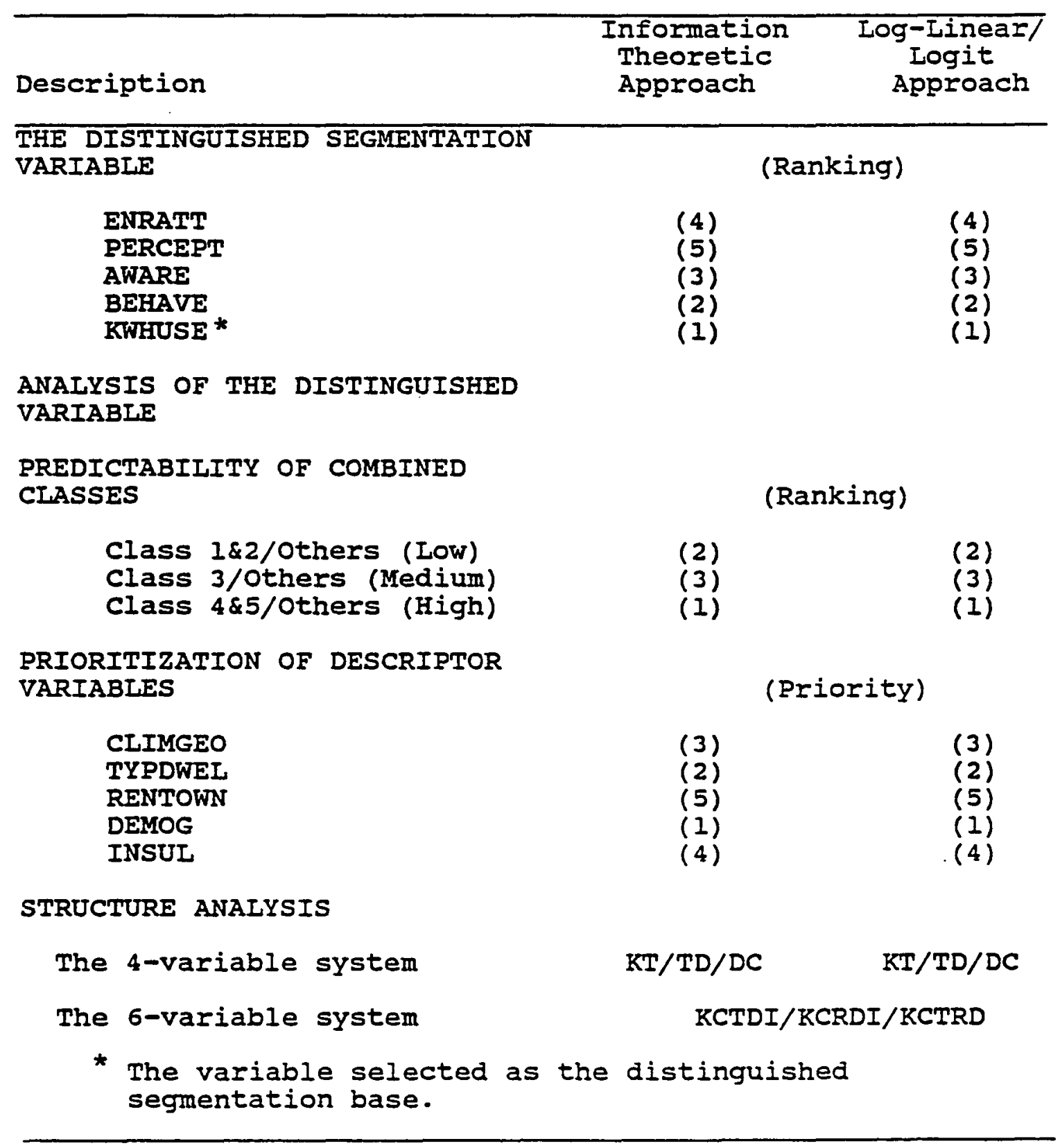




\section{CHAPTER V \\ CONCLUSIONS AND FUTURE DIRECTIONS}

This chapter presents the conclusions of the study as well as several suggestions for extensions and future directions in this area. First, a brief summary of the findings in this study is presented. Next, contributions of this study to (a) segment congruence analysis, (b) market segmentation in general, (c) log-linear modeling, and (d) information theoretic modeling are explored. Then, the limitations of this study are delineated. Finally, some unresolved problems and directions for further research are discussed.

\section{FINDINGS OF THE STUDY}

Multivariate segmentation studies are gaining in popularity due to the realization that, often, managerial judgment in selecting segmentation variables leads to incorrect definition and determination of market segments. one reason for this shortcoming is the dominant practice of identifying only one variable (e.g., usage rate) as the 
segmentation variable, and a class of this variable (e.g., the heavy user) as the target segment.

In reality, however, the choice of a segmentation variable, as well as a target segment, are context dependent. In the electrical utility industry, for example, the electricity consumption is viewed as a segmentation variable with respect to, say, the strategy of identifying and retaining the heavy users. On the other hand, attitudes toward energy conservation is a segmentation variable if, for instance, the utility company plans to implement a conservation program.

Some segmentation variables are more "accessible" than others. Knowledge about the characteristics of the individuals associated with the more accessible segmentation variable may lead to a better understanding of the other, less accessible, variables.

Segment congruence analysis is composed of a family of techniques which deals with multiple variable segmentation studies. The classical segment congruence analysis entailed only a few questions with the primary purpose of assessing mutual association among segmentation variables, assessing the contribution of each segmentation variable to the total mutual association, and assessing the predictability of an a priori selected distinguished variable.

The present study extended segment congruence analysis by introducing several relevant research questions. 
Furthermore, the information theoretic family of techniques was proposed as an approach useful for multivariate segmentation studies in the framework of segment congruence analysis. It was also demonstrated, both in the methodological and application sense, that the information theoretic approach is capable of addressing the old and the new questions in segment congruence analysis.

The results of this application, not only demonstrated the efficacy of the information theoretic and the log-linear approaches, but clearly demonstrated that the results of segment congruence analysis are meaningful to the practitioner as well as the researcher in market segmentation (In the next section of this chapter, contributions of this study will be discussed).

Tables XXXVI thru XXXIX outline all of the research questions in the two phases, the results of the PNWRES study application, and the practical and theoretical significance of these results. The assessment of mutual association and identification of candidate segmentation variables are given in Table XXXVI. The a priori selection of the distinguished variable and its analyses are given in Table XXXVII. The results of the "identification and analysis of the distinguished variable" using the analytical (as opposed to the a priori) approach, based on the endogenous and exogenous descriptor variables, are presented in Tables XXXVIII and XXXIX. 
TABLE XXXVI

PRACTICAI AND/OR THEORETICAI SIGNIFICANCE OF THE RESULTS OF SEGMENT CONGRUENCE ANALYSIS TO THE PNWRES STUDY: PHASE I

\begin{tabular}{|c|c|c|}
\hline Description & Result & Implications \\
\hline $\begin{array}{l}\text { RESEARCH OUESTION \#I } \\
\text { Are the segmentation } \\
\text { variables mutually } \\
\text { associated? If yes, } \\
\text { how can this mutual } \\
\text { association be } \\
\text { measured? } \\
\text { RESEARCH oUESTION \#2 }\end{array}$ & $\begin{array}{l}\text { YES } \\
\text { T/T } \\
0.0765\end{array}$ & $\begin{array}{l}\text { Knowledge about other } \\
\text { variables contributes to } \\
\text { the understanding of a } \\
\text { variable of particular } \\
\text { interest. }\end{array}$ \\
\hline $\begin{array}{l}\text { Which basis (or set } \\
\text { of bases) makes the } \\
\text { highest contribution } \\
\text { toward the mutual } \\
\text { association? } \\
\text { RESEARCH QUESTION \#3 }\end{array}$ & AWARE & $\begin{array}{l}\text { Based on this criterion, } \\
\text { energy related awareness } \\
\text { is a candidate for the } \\
\text { distinguished variable. } \\
\text { (Method \#I) }\end{array}$ \\
\hline $\begin{array}{l}\text { Which variable makes } \\
\text { the highest contribu- } \\
\text { tion toward the joint } \\
\text { variability in the } \\
\text { system of segmenta- } \\
\text { tion bases? } \\
\text { RESEARCH OUESTION } \# 4\end{array}$ & $\begin{array}{l}\text { (aware) }^{I} \\
\text { BEHAVE }\end{array}$ & $\begin{array}{l}\text { Based on this criterion, } \\
\text { energy related behavior } \\
\text { is a candidate for the } \\
\text { distinguished variable. } \\
\text { (Method } \# 2 \text { ) }\end{array}$ \\
\hline $\begin{array}{l}\text { Knowledge of which } \\
\text { variable makes the } \\
\text { highest contribution } \\
\text { toward the reduction } \\
\text { of overall uncert- } \\
\text { ainty in the overall } \\
\text { system of segmenta- } \\
\text { tion bases? } \\
\text { RESEARCH QUESTION \#5 }\end{array}$ & ENRATT & $\begin{array}{l}\text { Based on this criterion, } \\
\text { energy related attitudes } \\
\text { is a candidate for the } \\
\text { distinguished variable. } \\
\text { (Method \#3) }\end{array}$ \\
\hline $\begin{array}{l}\text { Which variable } \\
\text { has the most number } \\
\text { of statistically } \\
\text { significant dyadic } \\
\text { interrelationships } \\
\text { with other variables? }\end{array}$ & $\begin{array}{l}\text { PERCEPT } \\
\text { (aware) }^{2} \\
\text { (behave) }^{2}\end{array}$ & $\begin{array}{l}\text { Based on this criterion, } \\
\text { energy related percep- } \\
\text { tions is a candidate for } \\
\text { the distinguished vari- } \\
\text { able. (Method \#3) }\end{array}$ \\
\hline & KWHUSE & Key var \\
\hline
\end{tabular}

I BEHAVE, the second choice, was selected to increase the number of variables for this illustrative application.

2 All three variables had 3 significant dyadic relations. 
TABLE XXXVII

SIGNIFICANCE OF THE RESULTS OF SEGMENT CONGRUENCE ANALYSIS TO THE PNWRES STUDY

PHASE II: A PRIORI DISTINGUISHED VARIABLE

\begin{tabular}{|c|c|c|}
\hline Description & Result & Implications \\
\hline $\begin{array}{l}\text { RESEARCH QUESTION \#I } \\
\text { Which one of the } \\
\text { candidate distin- } \\
\text { guished segmentation } \\
\text { bases, identified in } \\
\text { phase I, is best } \\
\text { predicted once we } \\
\text { have the knowledge } \\
\text { of a set of relevant } \\
\text { independent variables? } \\
\text { RESEARCH oUESTION \#2 }\end{array}$ & $\begin{array}{l}\text { KWHUSE } \\
(10.998)\end{array}$ & selected a priori. \\
\hline $\begin{array}{l}\text { How much of the } \\
\text { uncertainty in the } \\
\text { distinguished vari- } \\
\text { able is reduced when } \\
\text { it is dichotomized } \\
\text { into a class (or } \\
\text { group of classes) } \\
\text { versus all of the } \\
\text { other classes? } \\
\text { RESEARCH OUESTION \#3 }\end{array}$ & $\begin{array}{l}11.18 \% \\
\text { (4\&5 vs. } \\
\text { others) } \\
6.45 \% \\
\text { (1\&2 vs. } \\
\text { others) } \\
6.10 \% \\
\text { ( } 3 \text { vs. } \\
\text { others) }\end{array}$ & $\begin{array}{l}\text { The classification of the } \\
\text { electricity consumption } \\
\text { variables to dichotomous } \\
\text { variables (light, medium, } \\
\text { or heavy users vs others) } \\
\text { shows the heavy user seg- } \\
\text { ment is best predicted by } \\
\text { the other segmentation } \\
\text { variables. }\end{array}$ \\
\hline $\begin{array}{l}\text { How can one priori- } \\
\text { tize the effect of } \\
\text { the independent } \\
\text { variables on the } \\
\text { distinguished set? }\end{array}$ & $\begin{array}{l}1: \text { ENRATT } \\
2: \text { PERCEPT } \\
3: \text { GENATT } \\
\text { 4: AWARE } \\
\text { 5: BEHAVE }\end{array}$ & $\begin{array}{l}\text { In reducing the number of } \\
\text { descriptor variables to } \\
\text { be used in subsequent } \\
\text { analyses, use the given } \\
\text { order (ENRATT=Most- } \\
\text { BEHAVE=least-important). }\end{array}$ \\
\hline $\begin{array}{l}\text { RESEARCH OUESTION \#4 } \\
\text { What is the depen- } \\
\text { dency structure in } \\
\text { each class of the } \\
\text { distinguished seg- } \\
\text { mentation base? }\end{array}$ & $\begin{array}{l}\text { KEPGA/ } \\
\text { ABK }\end{array}$ & $\begin{array}{l}\text { When four segmentation } \\
\text { variables are used, in- } \\
\text { direct/direct effects } \\
\text { in the simplest structure } \\
\text { are as given. } \\
\text { When all six segmentation } \\
\text { variables are used, in- } \\
\text { direct/direct effects } \\
\text { in the simplest structure } \\
\text { are as given. }\end{array}$ \\
\hline
\end{tabular}


TABLE XXXVIII

SIGNIFICANCE OF THE RESULTS OF SEGMENT CONGRUENCE ANALYSIS TO THE PNWRES STUDY

PHASE II: OTHER SEGMENTATION VARIABLES AS DESCRIPTOR VARIABLES (ANALYTICAL)

\begin{tabular}{|c|c|c|}
\hline Description & Result & Implications \\
\hline $\begin{array}{l}\text { RESEARCH OUESTION \#l } \\
\text { Which one of the } \\
\text { candidate distin- } \\
\text { guished segmentation } \\
\text { bases, identified in } \\
\text { phase I, is best } \\
\text { predicted once we } \\
\text { have the knowledge } \\
\text { of a set of relevant } \\
\text { independent variables? } \\
\text { RESEARCH oUESTION } \# 2\end{array}$ & $\begin{array}{l}\text { BEHAVE } \\
(73.84 \%)\end{array}$ & $\begin{array}{l}\text { When the other segmenta- } \\
\text { tion variables are used } \\
\text { as descriptors, energy } \\
\text { related behavior is best } \\
\text { predicted by these vari- } \\
\text { ables. }\end{array}$ \\
\hline $\begin{array}{l}\text { How much of the } \\
\text { uncertainty in the } \\
\text { distinguished vari- } \\
\text { able is reduced when } \\
\text { it is dichotomized in- } \\
\text { to a class (or group } \\
\text { of classes) versus all } \\
\text { of the other classes? } \\
\text { RESEARCH oUESTION \#3 }\end{array}$ & $\begin{array}{c}73.848 \\
\text { (Classes } \\
1 \& 2 \text { ) }\end{array}$ & $\begin{array}{l}\text { The classification of the } \\
\text { energy related behavior } \\
\text { to two classes of "action } \\
\text { taken/action not taken," } \\
73.848 \text { of uncertainty } \\
\text { regarding this variable } \\
\text { is removed. }\end{array}$ \\
\hline $\begin{array}{l}\text { How can one priori- } \\
\text { tize the effect of } \\
\text { the independent } \\
\text { variables on the } \\
\text { distinguished set? }\end{array}$ & $\begin{array}{l}1: \text { AWARE } \\
2: \text { KWHUSE } \\
3: \text { ENRATT } \\
\text { 4: PERCEPT } \\
5: \text { GENATT }\end{array}$ & $\begin{array}{l}\text { In reducing the number of } \\
\text { descriptor variables to } \\
\text { be used in subsequent } \\
\text { analyses, use the given } \\
\text { order (AWARE=Most- } \\
\text { GENATT=least-important). }\end{array}$ \\
\hline $\begin{array}{l}\text { RESEARCH QUESTION \#4 } \\
\text { What is the depen- } \\
\text { dency structure in } \\
\text { each class of the } \\
\text { distinguished seg- } \\
\text { mentation base? }\end{array}$ & KEPGA/ABK & $\begin{array}{l}\text { When four segmentation } \\
\text { variables are used, in- } \\
\text { direct/direct effects } \\
\text { in the simplest structure } \\
\text { are as given. } \\
\text { When all six segmentation } \\
\text { variables are used, in- } \\
\text { direct/direct effects } \\
\text { in the simplest structure } \\
\text { are as given. }\end{array}$ \\
\hline
\end{tabular}


TABLE XXXIX

SIGNIFICANCE OF THE RESULTS OF SEGMENT CONGRUENCE ANALYSIS TO THE PNWRES STUDY

PHASE II: EXOGENOUS DESCRIPTOR VARIABLES

\begin{tabular}{|c|c|c|}
\hline Description & Result & Implications \\
\hline $\begin{array}{l}\text { RESEARCH OUESTION \#I } \\
\text { Which one of the } \\
\text { candidate distin- } \\
\text { guished segmentation } \\
\text { bases, identified in } \\
\text { phase I, is best } \\
\text { predicted once we } \\
\text { have the knowledge } \\
\text { of a set of relevant } \\
\text { independent variables? } \\
\text { RESEARCH ouESTION \#2 }\end{array}$ & $\begin{array}{l}\text { KWHUSE } \\
(13.18 \%)\end{array}$ & $\begin{array}{l}\text { When the exogenous des- } \\
\text { criptor variables are } \\
\text { used, electricity con- } \\
\text { sumption is the best } \\
\text { predicted segmentation } \\
\text { variable. }\end{array}$ \\
\hline $\begin{array}{l}\text { How much of the } \\
\text { uncertainty in the } \\
\text { distinguished vari- } \\
\text { able is reduced when } \\
\text { it is dichotomized } \\
\text { into a class (or } \\
\text { group of classes) } \\
\text { versus all of the } \\
\text { other classes? }\end{array}$ & $\begin{array}{l}16.56 \% \\
\text { (4\&5 vs. } \\
\text { others) } \\
9.13 \% \\
\text { (1\&2 vs. } \\
\text { others) } \\
6.50 \% \\
\text { (3 vs. } \\
\text { others) }\end{array}$ & $\begin{array}{l}\text { The classification of the } \\
\text { electricity consumption } \\
\text { variables to dichotomous } \\
\text { variables (light, medium, } \\
\text { or heavy users vs others) } \\
\text { shows the light user seg- } \\
\text { ment is best predicted by } \\
\text { the exogenous variables. }\end{array}$ \\
\hline $\begin{array}{l}\text { RESEARCH OUESTION \#3 } \\
\text { How can one priori- } \\
\text { tize the effect of } \\
\text { the independent } \\
\text { variables on the } \\
\text { distinguished set? } \\
\text { RESEARCH oUESTION \#4 }\end{array}$ & $\begin{array}{l}1: \text { DEMOG } \\
2: \text { TYPDWEL } \\
3: \text { CLIMGEO } \\
4: \text { INSUL } \\
5: \text { RENTOWN }\end{array}$ & $\begin{array}{l}\text { In reducing the number of } \\
\text { descriptor variables to } \\
\text { be used in subsequent } \\
\text { analyses, use the given } \\
\text { order (DEMOG=Most- } \\
\text { RENTOWN=least-important). }\end{array}$ \\
\hline $\begin{array}{l}\text { What is the depen- } \\
\text { dency structure in } \\
\text { each class of the } \\
\text { distinguished seg- } \\
\text { mentation base? }\end{array}$ & $\begin{array}{l}\text { KCTDI/ } \\
\text { KCRDI/ } \\
\text { KCTRD }\end{array}$ & $\begin{array}{l}\text { When only four } \\
\text { variables are used, in- } \\
\text { direct/direct effects } \\
\text { in the simplest structure } \\
\text { are as given. } \\
\text { When all six } \\
\text { variables are used, in- } \\
\text { direct/direct effects } \\
\text { in the simplest structure } \\
\text { are as given. }\end{array}$ \\
\hline
\end{tabular}


These results entail several implications for managers at BPA or other utility companies. These implications are exemplified under the context of identifying different segments on the basis of electricity consumption as follows:

- Electricity consumption is significantly interrelated with attitudes and energy related perceptions, awareness, and behavior.

- Electricity consumption (in general! is somewhat better predicted by exogenous descriptor variables (13.18\%), (i.e., demographics of the residents, type of dwelling unit, geoclimatic environment, level of insulation of the residence, and rental/ownership status of the residence) than by the other segmentation variables $(10.99 \%)$.

- The high electricity user is better predicted by the exogenous variables (16.56\%) as compared with the medium and light users (6.50\% and $9.13 \%$, respectively).

- Demographic characteristics of the residents, type of the residential unit, and the geoclimatic variables are the highest contributors to the knowledge of electricity consumption among the exogenous variables considered.

These, and similar interpretations of the segment congruence analysis will enhance the marketing manager's understanding of the issues surrounding segmentation studies. The ultimate decision, of which segment(s) to select for targeting purposes, is, of course up to the marketing manager. 
CONTRIBUTIONS OF THE STUDY

The present study resulted in contributions in several areas including (a) segment congruence analysis, (b) market segmentation (c) log-linear modeling, and (d) information theoretic modeling. These contributions are examined in this section.

\section{Contribution to Segment Congruence Analysis}

Marketing practitioners should recognize that in many markets it is necessary to refine their definition of market segments in order to be able to serve more efficiently and effectively. To do so, they must recognize that segmentation based on a single, a priori selected variable will often result in vague and inaccurately defined (set of) segment(s). On the other hand, in many cases, segmentation variables are a set of discrete (or discretized) variables for which data has been collected on dichotomous or polytomous scales.

The above two characteristics of modern market segmentation (i.e., multiple variables and categorical data) bring about the need for techniques for analyzing such data (i.e., discrete multivariate analysis techniques). The study of the interrelations between a set of segmentation variables has been termed segment congruence analysis. Contemporary marketing researchers and practitioners have 
advocated a variety of approaches to such analyses. However, they have stopped after what can be considered an exploratory study of multiple segmentation variables (Green and Carmone 1977, Van Auken and Ionial 1984).

The present study extends segment congruence analysis to include several other important aspects of discrete multivariate analysis. This extension includes:

- a number of alternative methods of identifying candidate segmentation variables,

- objective identification of a distinguished segmentation variables (using endogenous or exogenous descriptor variables).

- prioritization of the descriptor variables with respect to their contribution to the knowledge about the segmentation variable, and

- structural modeling of the system of segmentation and descriptor variables with the purpose of understanding the nature and magnitude of interrelations among them.

These additional questions have enhanced segment congruence analysis from a method of exploratory analysis of systems of multiple segmentation variables to a descriptive study of such systems. Particularly, the addition of structural modeling techniques addresses the nature of interrelations among these variables.

Furthermore, the introduction of exogenous descriptor variables has enabled segment congruence analysis to be applied to an actual market segmentation study. In this study, the structure of the market is studied, not only to identify a distinguished segmentation variable, but, to 
describe the relation of this variable to a set of background variables.

\section{Contribution to Market segmentation}

Contributions of this research to the general area of market segmentation is threefold. First, as mentioned before, by introducing a set of exogenous descriptor variables, segment congruence analysis can be viewed as an integral part of market segmentation. Any segmentation study, a priori, a posteriori, or hybrid, can use phase II of this study.

second, a posteriori and hybrid market segmentation studies can take advantage of the methods proposed for identifying candidates for the distinguished segmentation variable. Several researchers including Green (1977), wind (1978), and others have expressed concern about a posteriori segmentation studies with respect to the meaningfulness of their results. A segmentation variable identified by clustering based techniques may produce highly significant results analytically, but completely anomalous results practically.

The present study, can not, of course, claim the prevention of such results. However, it can help to identify a more meaningful practical (as well as analytical) segmentation variable by proposing a number of them as the 
candidates to be evaluaced for the distinguished segmentation variable.

Third, by introducing the information theoretic approach to segment congruence analysis, this study has provided a powerful tool for analysis of discrete multivariate systems, which is extremely useful for modern market segmentation analysis. Information theory has already been used to develop brand-choice models by Herniter (1973 and 1974) and Bass (1976) among others. This study introduce this approach to market segmentation studies.

\section{Contribution to Log-Linear Modeling}

In its more than four decades of history, the development of information theory has enabled the formulation of many interesting managerial concerns in the form of easy-to-understand expressions and procedures. Loglinear models in their current form are relatively new, therefore, many models, with managerial implications, have not been developed explicitly, in spite of these techniques ability to address them. Furthermore, some of the commonly used models have been misinterpreted with respect to their meaning and the hypotheses tested.

Two misinterpretations of the log-linear models, which were encountered in this study are discussed next. This discussion is followed by two log-linear based procedures to address the fifth research questions in both phases. 
The second research question in the first phase (i.e., contribution of each segmentation variable to the overall mutual association) has, traditionally, been assessed using the one-variable independence model $(e . g .,\{A\}\{B C D .$.$\} . It$ was shown, in this study, that the correct model for this research question is the mutual independence model minus the two-variable independence models (i.e., \{ABCD...\}$\{A B\}\{A C\} . .$.$) . This was done by comparing the two models$ with the information theoretic expression used for this question (i.e., $\left.T_{A}(B C D . .)=.T(A: B: C: \ldots)-T(A: B)-T(A: C)-\ldots\right)$. This was obviously a case of misinterpreting the log-linear models.

It was then shown that the one-variable independence model addresses the third research question in the first phase (i.e., the contribution of each variable to the joint variability in the system of segmentation variables). This, again was initially discovered by the fact the information theoretic model --e.g., $S(A B C . .)-.S_{A}(B C . .$.$) , generated$ identical statistics to the one-variable independence model.

The second misinterpretation relates to the third research question in the second phase (i.e, prioritize the effect of the descriptor variables on the distinguished variable). Green and Carmone (1977), briefly, claim that the coefficients of the logit model can be used to prioritize the effect of the independent variables on the dependent variable variable. This is true only in the case 
of all dichotomous independent variables. In this case each variable will have only one coefficient. (By analogy to the more commonly known dummy variable regression models, each dichotomous independent variable will have one dummy variable in the model).

However, in the case of polytomous independent variables, the model will produce several coefficients for each variable, which are absent or present, depending on which classes of the dependent variable are being considered. This problem was resolved by developing loglinear models (equivalent to Abrahamse and van Bueren, 1980) to assess the contributions of the descriptor variables to the segmentation variable.

Two log-linear procedures were developed to address two other research questions. The first procedure, which was used in addressing the $f$ ifth research question in the first phase, involved developing successive bivariate loglinear models. These models were, then, summarized in a two dimensional table (this table was, subsequently used to identify the variable with the highest number of dyadic interactions with other variables).

The second procedure was developed to construct equivalent models to Conant's (1981 and 1982) dependency analysis. The contributions of the other variables to each variable were assessed and a prioritization scheme similar to that of Abrahamse and van Bueren's (1980) was developed 
for each variable. Then, these prioritizations were used to construct the approximate structure for the multivariate system. This approach, of course, produced the same exact structural model for the data.

Contribution to Information Theoretic Modeling

Information theoretic models have been used in economics, psychology, sociology, business administration, and other social sciences. However, most of these applications, particularly those to business administration (e.g., accounting (Lev 1968, 1970), finance (Philipatos and Gressis 1975), management (Horowitz and Horowitz 1976), and marketing (e.g., Herniter 1973 and 1974, Bass 1976)), have largely been simple applications of constraint analysis. This study applies models as simple as entropy and transmission to more complicated models, such as dependency analysis.

This study represents the first application of information to segment congruence analysis. It also represents the first in-depth application of this technique to any segmentation problem. Furthermore, this research project is among the very few undertakings in marketing which utilize information theoretic models.

It is through such applications and comparisons that a researcher can understand and appreciate the efficacy of an approach. Information theory seems to demonstrate 
considerable capability in addressing the research questions addressed throughout this study.

\section{IIMITATIONS}

As any other research project, this study has certain limitations. These limitations, however, should not provide a hindrance in realizing the benefits provided by the proposed methodology.

This study is methodological, and therefore, emphasizes the techniques used, rather than the relevance of the application and the particular data analyzed (though, the analysis produced plausible results). The strongest motives for selecting the PNWRES database were the relevance of the data to the segment congruence issue, availability of the data, the large sample size, and the large number of variables measured.

Since the PNWRES data were not collected specifically for market segmentation purposes, some limitation arise. For instance, the survey was far more oriented toward the residences and their characteristics than toward the consumers. Although the residence descriptors are thought to be important precursors of individuals energy related behavior, a more detailed description of consumer characteristics would have been useful. 
All variables used in an application oriented research project must be examined for reliability and scrutinized for validity. Validity and reliability issues were not addressed, as the purpose of this study was not to assess issues in the energy industry per se, but to demonstrate the relevance of the research questions and the methodology through a readily available data set. On the other hand, since many methodological studies use data which are (a) hypothetical (i.e., contrived) and/or (b) contain small number of observations, the 1983 PNWRES data had the added advantage of being an actual survey with 4703 cases. The results of this study, therefore, are far more indicative of the efficacy of the methods used.

Finally, the only means of the initial variable selection for the study was expert opinion. Initially a large number of variables were selected (a priori) as potential segmentation and descriptor variables. These variables were presented to the executives at BPA and PP\&L. Their recommendations were accommodated and the final list of variables was compiled.

Again, it is emphasized that the analysis provided an excellent demonstration for the efficacy of the techniques used. Furthermore, the results of the application did not seem to produce counter-intuitive conclusions despite the mentioned Iimitations. 
DIRECTIONS FOR FURTHER RESEARCH

Several directions for further research can readily be identified. These directions can be categorized under (1) segment congruence analysis, (2) log-linear modeling, information theoretic modeling, and (4) other.

segment congruence analysis is a relatively new area in market segmentation. The present study attempted to develop an exploratory and, to an extent, a descriptive approach to segment congruence analysis. The natural continuation of this stream of research would involve development of models for predictive purposes. Both information theory and log-linear models offer rich prospects in this area. Also, an application oriented research project, with primary data collection, is needed in order to further establish segment congruence analysis as a practical and valuable tool.

Log-linear modeling is relatively young, particularly, in the area of structural modeling. Both researchers and practitioners would benefit from further development in this area. Continuing with comparisons between better known models, such as those offered by information theory, would provide an excellent stream of research. Information theory contains models that deal with phenomena in the context of Bayseian statistics. It would probably be fruitful to develop the log-linear approach to such models. 
Information theoretic models from simple models of marginal and conditional entropies, transmissions, interactions, and systematic entropies to the complex models for spectral analysis, dependency analysis and reconstructability analysis, offer a rich family of techniques applicable to many business problems. The bridge between business application and information theory must be strengthened and maintained. This would set the stage for synergistic developments in business problem solving and statistical modeling.

Information theoretic structural modeling was proposed by Ashby (1964) and, more recently, further developed by Klir. and and his colleagues, and several other researchers including Broekstra, Krippendorff, and conant. The present study employed Conant's (1981, 1982) dependency analysis. The other methods for structural modeling, proposed by these authors, can be incorporated in further development of segment congruence analysis.

Finally, it is highly desirable to develop an "expert system" for segment congruence analysis through the two methodologies discussed in this study. In this era of information explosion and machine intelligence, developing such a system would help reduce the immense complexity surrounding managers and decision makers, as well as taking advantage of the emerging power of machines. 


\section{BIBLIOGRAPHY}

Abrahamse, A.P.G., and W.M. Lammerts van Bueren (1980) "Nominal Variables: Dependence and Predictive Power," Report $8017 / \mathrm{S}$, Economic Institute, Erasmus University Rotterdam.

Alpert, M.I. (1971) "A Canonical Analysis of Personality and the Determinants of Automobile Choice," Proceedings, American Marketing Association, 312-316.

Andrews, F.M., and R.C. Messenger (1973) Multivariate Nominal Scale Analysis, Ann Arbor, Michigan: Survey Research Center, University of Michigan.

Andrews, Frank M., Morgan James N., and Sonquist John A., (1969) Multiple Classification Analysis, Survey Research Center, Institute for Social Research, The University of - Michigan, Ann Arbor, Michigan.

Arabie, P. (1977) "Clustering Representations of Group Overlap," Journal of Mathematical Sociology, 5, 113-28.

Arabie, P., J.D. Carroll, W. DeSarbo, and J. Wind (198I) "Overlapping Clustering: A New Method for Product Positioning," Journal of Marketing Research, XVIII, (August) 310-17.

Armstrong, J.S., and J.G. Anders (1970) "Exploratory Analysis of Markeiing Data: Trees vs. Regression," Journal of Marketing Research, VII, (November) 487-492.

Ashby, W.R. (1964) "Constraint Analysis of Many-valued Relations," General systems, 9, 99-105.

Assael, H., (1970) "Segmenting Markets by Group Purchasing Behavior: An Application of AID Technique," Journal of Marketing Research, VII, (May) 153-158.

Attneave, F. (1959) Applications of Information Theory to Psychology: A Summary of Basic Concepts, Methods, and Results, New York: Holt, Rinehart and Winston. 
Bartlett, M.S. (1955) An Introduction to stochastic Processes, Cambridge Univ. Press.

Bass, F.M. (1976) "The Theory of Stochastic Preference and Brand Switching," Journal of Marketing Research, XI (February) 1-20.

Batsell, R.R. (1980) "Consumer Resource Allocation Models at the Individual Level," Journal of Consumer Research, 7 (June) 78-87.

Batsell, R.R., and L.M. Lodish (198I) "A model and Measurement Methodology for Predicting Individual Consumer Choice," Journal of Marketing Research, 18 (February) 1-12.

Bernhardt, I. and K.D. Mackenzie (1968) "Measuring Seller Unconcentration, Segmentation and Product differentiation," Western Economic Journal, 6 (December) 395-403.

Bernhardt, K.I., and T.C. Kinnear (1976) "Categorical Regression in Marketing," Journal of Business Research, 4. (November) 297-312.

Best, R.J. (1975) The Relationship Between Joint-Space Distance and stochastic Choice: An Empirical Investigation, Ph. D. Dissertation, University of oregon, Eugene, Oregon.

Bishop, Y.M.M. (1969) "Full Contingency Tables, Logits, and split contingency Tables," Biometrics, (June) 383-399.

Bishop, Y.M.M., S.E. Fienberg, and P.W. Holland (1975) Discrete Multivariate Analysis: Theory and Practice, Cambridge, Mass.: The MIT Press.

Blalock, Hubert M., (1972) Social Statistics, McGraw-Hill Book Company.

Blattberg, R., and S. Sen (1976) "Market segmentation and Stochastic Brand Choice Models," Journal of Marketing Research , XIII, (February) 34-45.

Brillouin, L. (1956) Science and Information Theory, New York: Academic Press.

Broekstra, G. (1978) "On the Representation and Identification of structure systems," International Journal of Systems Science, 9, 1271-1293. 
Broekstra, G. (1981) "C-Analysis of C-structures: Representation and Evaluation of Reconstruction Hypotheses by Information Measures," International Journal of systems, 7, 33-6I.

Broekstra, G. (1982) "Constraint Analysis of Discrete Multivariate Systems," Proceedings 26th Annual Meeting of the Society for General Systems Research, Washington D.C., 259-265.

Caval1o, R.E. and G.J. Klir (1979) "Reconstructability Analysis of Multi-Dimensional Relations: A Theoretical Basis for Computer-Aided Determination of Acceptable Systems Models," International Journal of General Systems, 5, 143-171.

Cavallo, R.E. and G.J. Klir (1981) "Reconstructability Analysis: Evaluation of Reconstruction Hypotheses," International Journal of General Systems, 7, 7-32.

Chapman, R.G. and R. Staelin (1982) "Exploiting Rank Ordered Choice set Data Within the Stochastic Utility Model," Journal of Marketing Research, 19 (August) 288-301.

Cherry, E.C. (1952) "The Communication of Information", American Scientist, 40, 640-664.

Clogg, C.C. and J.M. Munch (1984) "Using Simultaneous Latent Structure Models to Analyze Group Differences:

Exploratory Analysis of Buying style Items," Journal of Business Research, 12, 319-336.

Clogg, C.C., and L.A. Goodman, (1985) "Simultaneous Latent Structure Analysis in Several Groups," Sociological Methodology, 59, 124-38.

Coleman, J.S. (1964) Introduction to Mathematical Sociology, New York: Free Press.

Conant, R.C. (1974) "Information Flows in Hierarchical systems," International Journal of General Systems, 1, 918 .

Conant, R.C. (1981) "Detection and Analysis of Dependency Structures," International Journal of General Systems, 7, 81-91.

Conant, R.C. (1982) "Approximating General structures with Dependency Analysis," Proceedings 26rd Annual Meeting of the Society for General Systems Research. 
Cramer, H. (1946) Mathematical Methods of Statistics, Princeton Univ. Press.

Currim, I.S., (1981) "Using Segmentation Approaches for Better Prediction and Understanding from Consumer Mode Choice Models," Journal of Marketing Research, 18 (August) 301-9.

Currim, I.S., (1982) "Predictive Testing of Consumer Choice Models That Are Not Subject to Independence of Irrelevant Alternatives," Journal of Marketing Research, 19 (May) 208-22.

Darroch, J.N. (1962) "Interactions in Multifactor Contingency Tables," Journal of the Royal statistical Society, 24, 251-263.

Davis, J.A., (1975) "Analyzing Contingency Tables with Iinear Flow Graphs: D Systems," In Sociological Methodology 1976, edited by D.R. Heise, 111-45. San Francisco: Jossey-Bass.

Dempster, A.P., N.M. Laird, and D.B. Rubin (1977) "Maximum Iikelihood From Incomplete Data Via the EM algorithm," Iournal of the Royal statistical Society, Series B, 39, $1-38$.

Desarbo, W.S., and D.K. Hildebrand (1980) "A Marketer's Guide to Log-Linear Models for Qualitative Data Analysis," Journal of Marketing, (Summer) 44, 40-51.

Fisher, R.A. (1925) "Theory of statistical Estimation," Proceedings of Cambridge Philosophical society, 22, 700725.

Flath, D. and E.W. Leonard (1979) "A Comparison of Two Logit Models in the Analysis of Qualitative Marketing Data," Journal of Marketing Research, 16 (November) 533-38.

Frank, R.E., W.F. Massy, and Y. Wind, (1972) Market Segmentation, Prentice-Hall, Inc., Englewood Cliffs, N.J.

Friedlander, D. (1961) "A Technique for Estimating a Contingency Table Given the Marginal Totals and Some Supplementary Data," Journal of the Royal statistical Society, $124,412-420$.

Fritzsche, D.J. (1981) "An Analysis of Energy Consumption Patterns by Stage of Family Life Cycle," Journal of Marketing Research, XVIII (May) 227-232. 
Garner, W.R. (1962) Uncertainty and Structure as Psychological concepts, New York: John Wiley and Sons, Inc.

Garner, W.R. and W.J. McGill (1956) "The Relation Between Information and Variance Analysis, Psychometrika, 21 (September) 219-228.

Gensch, D.H. and W.W. Recker (1979) "The Multinomial, Multiattribute Logit Choice Model," Journal of Marketing Research, 16 (February) 124-32.

Goodman, I.A, (1963) "On Methods for Comparing Contingency Tables," Journal of the Royal statistical society, Series A, 126, 94-108.

Goodman, I.A, (1964a) "Simultaneous Confidence Limits for Cross-Product Ratios in Contingency Tables," Journal of the Royal Statistical Society, Series B, 26, 86-102.

Goodman, I.A, (1964b) "Simple MEthods for Analyzing ThreeFactor Interaction in contingency Tables," Journal of the American Statistical Association, 59, 319-52.

Goodman, L.A, (1964c) "Interactions in Multidimensional Contingency Tables," Annals of Mathematical statistics, $35,632-46$.

Goodman, I.A, (1964d) Simultaneous Confidence Intervals for Multinomial Populations," Annals of Mathematical statistics, $35,716-25$.

Goodman, I.A, (1965a) "On Simultaneous Confidence Intervals for Multinomial Proportions," Technometrics, 7, 247-54.

Goodman, L.A, (1965b) "On the Statistical Analysis of Mobility Data," American Journal of Sociology, 70, 56485 .

Goodman, I.A, (1965c) "On the Multivariate Analysis of Three Dichotomous Variables," American Journal of Sociology, 7I, 290-30I.

Goodman, I.A, (1969a) "How to Ransack Social Mobility Tables and other kinds of Cross-Classification Tables," American Journal of Sociology, $75,1-40$.

Goodman, L.A, (1969b) "On Partitioning $x^{2}$ and Detecting Partial Associations in Three-Way Contingency Tables," Journal of the Royal statistical society, series B, 31, 486-98. 
Goodman, I.A, (1970) The Multivariate Analysis of Qualitative Data," Journal of the American statistical Association, 65, 226-56.

Goodman, I.A. (1971a) "The Analysis of Multidimensional Contingency Tables: Stepwise Procedures and Direct Estimation Methods for Building Models for Multiple Classifications," Technometrics, 13, 33-61.

Goodman, I.A, (1971b) "Partitioning of Chi Square, Analysis of Marginal Contingency Tables, and Estimation of Expected Frequencies in Multidimensional Contingency Tables," Journal of the American statistical Association, $66,339-44$.

Goodman, L.A. (1968) "The Analysis of Cross-Classified Data: Independence, Quasi-Independence, and Interactions in Contingency Tables with or without Missing Entries," Journal of the American statistical Association, 63, $1091-1131$.

Goodman, L.A. (1972) "A General Model for the Analysis of Surveys," American Journal of Sociology, 77, 1035-1087.

Goodman, I.A, (1973) "The Analysis of Multidimensional Contingency Tables When Some Variables are Posterior To Others: A Modified Path Analysis Approach, Biometrica, $59,579-96$.

Goodman, I.A., (1974) "Exploratory Latent Structure Analysis Using Both Identifiable and Unidentifiable Models," Biometrika, 61, 215-31.

Goodman, L.A. (1978) Analyzing Qualitative/Categorical Data, Edited by $J$. Magidson, Abt Books, Cambridge, Massachussettes.

Green, P.E. (1977) "A New Approach to Market Segmentation," Business Horizons, (February) 61-73.

Green, .P.E. (1978) "An AID/Logit Procedure for Analyzing Large Multiway Contingency Tables," Journal of Marketing Research, XV, (February) 132-36.

Green, P.E. and F.J. Carmone (1977) "Segment Congruence Analysis: A Method for Analyzing Association Among Alternative Bases for Market Segmentation," Journal of Consumer Research, 3 (March) 217-222.

Green, P.E., and V.R. Rao (1972) Applied Multidimensional Scaling: A Comparison of Approaches and Algorithms, New York: Holt, Rinehart and Winston. 
Green, P.E., F.J. Carmone, and D.P. Wachspress (1976) "Consumer Segmentation Via Latent Class Analysis," Journal of Consumer Research, 3 (December) 170-174.

Green, P.E., F.J. Carmone, and D.P. Wachspress (1977) "On the Analysis of Qualitative Data in Marketing Research," Journal of Marketing Research, XIV (February) 52-59.

Grizzle, J.E., C.F. Stamper, and G.G. Koch, (1969) Analysis of Categorical Data by Linear Models," Biometrics, 25, 489-504.

Haberman, S.J. (1972) "Iog-linear Fit for Contingency Tables," Applied statistics, 21, 218-25.

Haberman, S.J. (1982) "Analysis of Dispersion of Multinomial Response," Journal of the American statistical Association, 77, No. 379 (September) 568-580.

Harmon, R.R., J. Hosseini, and M. Zwick (1986) "Application of Information Theory to the Analysis of Market Segments when Multiple Segmentation Variables Are Used," Proceedings, American Marketing Association, Educators Meeting, Annual Conference, (August).

Haberman, S.J. (1979) Analysis of Qualitative Data, 1\&2, New York: Academic Press.

Herniter, J.D. (1973) "An Entropy Model of Brand Purchase Behavior," Journal of Marketing Research, X (November) 361-375.

Herniter, J.D. (1974) "A Comparison of the Entropy Model and the Hendry Model," Journal of Marketing Research, XI (February) 21-29.

Horowitz, A.R. and I. Horowitz (1976) "Education: The Real and Illusory Virtues of Entropy-Based Measures for Business and Economic Analysis," Decision sciences, 7, $121-136$.

Hosseini, J.C., R.R. Harmon, (1986) "Analyzing Overlapping Market Segments: An Information Theoretic Approach," Proceedings, Western American Institute for Decision Sciences, winter Conference, (March).

Hosseini, J.C., R.R. Harmon, and M. Zwick (1986) "Segment Congruence Analysis Via Information Theory," Proceedings, Society for General Systems Research, Annual Conference, (May) 
Hauser, J.R., and G.I. Urban (1977) "A Normative Methodology for Modeling Consumer Response to Innovation," operations Research, 25 (July-August) 579-619.

Jones, J.M. and F.S. Zufryden (1980) "Adding Explanatory Variables to a Consumer Purchase Behavior Model: An Exploratory Study," Journal of Marketing Research, 17 (August) 323-34.

Klir, G.J. (1976) "Identification of Generative Structures in Empirical Data," International Journal of General Systems, 3, 89-104.

Klir, G.J. (1986) "Third W. Ross Ashby Memorial Lecture 1986. Reconstructability Analysis: An offspring of Ashby's Constraint Analysis," Systems Research, 3, No. 4, 267-271.

Knoke, D., and P.J. Burke (1980) "Log-Iinear Models," Sage University Paper series on Quantitative Applications in the Social Sciences, 07-020, Beverly Hills and London: Sage Publications.

Krippendorff, K. (1979) "On the Identification of Structures in Multivariate Data by the spectral Analysis of Relations," Proceedings 23rd Annual Meeting of the Society for General Systems Research, Austin, Texas.

Krippendorff, K. (198I) "An Algorithm for Identifying structural Models of Multi-Variate Data," International Journal of General Systems, 7, 63-79.

Krippendorff, K. (1986) "Information Theory: Structural Models for Qualitative Data," Sage University Paper Series on Quantitative Applications in the Social Sciences, 07-062, Beverly Hills and London: Sage publications.

$\mathrm{Ku}, \mathrm{H.H}$. and S. Kullback (1968) "Interaction in Multidimensional Contingency Tables: An Information Theoretic Approach," Journal of Research of the National Bureau of standards, 72B (July-september) 159-99.

Kullback, s. (1959) Information Theory and Statistics, New York: John Wiley and Sons, Inc. 
Iagarce, R. (1974) "An Analysis of Second-order Interaction in Multidimensional Contingency Tables," Journal of Marketing Research, 11 (August) 343-45.

Lev, B. (1968) "The Aggregation Problem in Financial Statements: An Information Approach," Journal of Accounting Research, 6, (Autumn) 247-61.

Lev, B. (1970) "The Informational Approach to Aggregation in Firancial Statements: Extensions," Journal of Accounting Research, 8, (Spring) 78-94.

Lazarsfeld, P.F. and N.W. Henry (1942) Latent Structure Analysis, Boston, Houghton Mifflin Company.

Lewis, B.N., (1962) "On the Analysis of Interaction in Multi-Dimensional Contingency Tables," Journal of Royal Statistical Society, Series A, 125, 88-117.

Iilien, G.I. and P. Kotler (1983) Marketing Decision Making: A Model-Building Approach, New York: Harper and Row.

Malhotra, N.K. (1982a) "Information Load and Consumer Decision Making," Journal of Consumer Research, 8 (March) 419-30.

Malhotra, N.K. (1982b) "Structural Reliability and stability of Non-Metric Conjoint Analysis," Journal of Marketing Research, 19 (May) 199-207.

Malhotra, N.K., A.K. Jain, and S. Lagakos (1982) "The Information Load Controversy: An Alternative viewpoint," Journal of Marketing, 46 (Spring) 27-37.

Martin, C.R., and R.I. Wright (1974) "Profit Oriented Data Analysis for Market Segmentation: An Alternative to AID," Journal of MArketing Research, XI, (August) 399-412.

McGill, พ.J. (1954) "Multivariate Information Transmission," Psychometrica, 19, 97-116.

Miller, G.A. (1953) "What is Information Measurement?," American psychologist, 8, 3-11 
Miller, J., and M. Erickson, (1974) "On Dummy Variable Regression Analysis: A Description and Illustration of the Method," Sociological Methods and Research, 2, 40930 .

Mitra, S.K. (1955) "Contributions to the statistical Analysis of Categorical Data," N.C. Institute of statistics, Mimeo Series No. 142 (December).

Morgan James N. and Messenger Robert C., (1973) THAID: $\underline{A}$ Sequential Program for analysis of Nominal scale Dependent variables, Survey Research Center, Institute for Social Research, The University of Michigan, Ann Arbor, Michigan.

Myers, J.G. and F.M. Nicosia (1967) "New Empirical Directions in Market Segmentation: Latent structure Models", AMA Winter Conference Proceedings, Chicago, I11.: American Marketing Association.

Newman, J.W., and R. Staelin (1971) "Multivariate Analysis of Differences in Buyer Decision Time," Journal of Marketing Research, VIII, (May) 192-8.

Pearson, K. (1904) "Mathematical Contributions to the Theory of Evolution, XIII, on the Theory of Contingency and Its Relation to Association and Normal Correlation, "Drap. Co. Mem. Biom. Ser., 1 .

Perreault, Jr., W.D. and H.C. Barksdale, Jr. (1980) "A Model-Free Approach for Analysis of Complex Contingency Data in Survey Research," Journal of Marketing Research, XVII (November) 503-15.

Perreault, Jr., W.D., D.N. Behrman, and G.M. Armstrong (1979) "Alternative Approaches for Interpretation of Multiple Discriminant Analysis in Marketing Research," Journal of Business Research, 7, 151-173.

Peters, W.H. (1970) "Using MCA to Segment New Car Markets," Journal of Marketing Research, VII, (August) 360-3. 
Philippatos, G.C., and N. Gressis (1975) "Conditions of Equivalence Among $E-V, S S D$, and $E-H$ Portfolio Selection Criteria: The Case for Uniform, Normal and Lognormal Distributions," Management Science, 21, (February) 6I725.

Punj, G.N., and R. Staelin (1978) "The Choice Process for Graduate Business Schools," Journal of Marketing Research, 15 (November) 588-98.

Rao, C.R. (1952) Advanced Statistical Methods in Biometric Research, New York: John Wiley and Sons.

Rao, C.R. (1965) "Criteria of Estimation in Large Samples," 345-362 in Contributions to Statistics, New York: Pergamon Press.

Rao, V.R. and F.W. Winter (1978) "An Application of the Multivariate Probit Model to Market Segmentation and Product Design," Journal of Marketing Research, XV (August) 361-368.

Rao, V.R. and H.S. Solgaard (1977) "A Comparison of Qualitative Response Models of Consumer Choice," AMA Educator's Proceedings, Chicago, Ill.: American Marketing Association, 135-140.

Reynolds, H.T. (1977) "Analysis of Nominal Data," Sage University Paper Series on Quantitative Applications in the Social Sciences, 07-007, Beverly Hills and London: Sage Publications.

Roy, S.N. and M.A. Kastenbaum (1955) "A Generalization of Analysis of Variance and Multivariate Analysis to Data Based on Frequencies in Qualitative Categories or Class Intervals," N.C. Institute of statistics, Mimeo series No. 131 (June 1).

Russell, S.W. and M.J. Rayan (1979) "Analyzing CrossClassification Data: An Improved Method for Predicting Events," Journal of Marketing Research, XVI, (November) 539-544.

Shannon, C.E. and $W$. Weaver (1949) The Mathematical Theory of Communication, University of Illinois Press, Urbana, Chicago, London. 
Silk, A.J., and G.I. Urban (1978) "Pre-Test-Market Evaluation of New Packaged Goods: A Model and Measurement Methodology," Journal of Marketing Research, 15 (May) 171-91.

Simpson, C.H., (1951) "The Interpretation of Interaction in Contingency Tables," Journal of Roval statistical Society, Series B, 13, 238-41.

Smith, W.R. (1956) "Product Differentiation and Market Segmentation as Alternative Marketing strategies," Journal of Marketing, (July) 21, 3-8.

SPSS ${ }^{X}$ Inc., (1986) SPSS $^{x}$ User's Guide, 2nd Ed., McGraw-Hill Book Company.

Stouffer, S.A., E.A. Schuman, L.C. Devinney, S.A. Star, and R.M. Williams, Jr. (1949) The American Soldier: Adjustments During Army Life. studies in Social Psychology in World War II, 1, Princeton N.J.: Princeton University Press.

Stumpers, F.I.H.M. (1953) "A Bibliography of Information Theory; Communication Theory-Cybernetics," R.I.E., M.I.T. (February 2) IRE Trans., PGIT-2 (November).

Theil, H. (1967) Economics and Information Theory, Chicago: Rand McNally and Company.

Theil, H. (1970) " On the Estimation of Relationships Involving Qualitative Variables," American Journal of Sociology, 76, 103-154

U.S. Department of Energy, Bonneville Power Administration, (1983) PNWRES User's Guide, (Spring).

Van Auken, S., and S.C. Lonial (1984) "Assessing Mutual Association Between Alternative Market Segmentation Bases," Journal of Advertising, 13, No. 1, 11-16.

von Bertalanffy, L. (1968) General System Theory: Foundations, Development, Applications, New York, George Braziller.

Watanabe, S. (1969) knowing and Guessing, John Wiley and Sons, Inc.

Wilks, S.S. (1935) "The Iikelihood Test of Independence in Contingency Tables," Annals of Mathematical

Statistics, 6, 190-196. 
Wilks, S.S. (1943) Mathematical Statistics, Princeton Univ. Press.

Wilner, D.M., R.P. Walkley, and S.W. Cook (1955) Human Relations in Interracial Housing: A Survey of the Contact Hypothesis, Minneapolis: University of Minnesota Press.

Wind, Y. (1978) "Issues and Advances in Segmentation Research," Journal of Marketing Research, XV, (August) ; 317-337.

Yule, G.U. and M.G. Kendall (1937) An Introduction to the Theory of Statistics, llth ed., Iondon: Charles Griffin.

Zeisel, H. (1968) Say it with Figures, Fifth Edition, Revised, New York: Harper \& Row.

Zwick, M. (1979) "Information Theoretic Definition of "Leading Part"," Working Paper, System Science Program, Portland state University. 
APPENDIX I

GENERAL LOG-IINEAR MODELS

There are two types of log-linear models; the general log-linear model and the logit model. In the general loglinear model variables are treated similarly with respect to their interdependence (i.e., no distinction is made based on dependence/independence between variables). In the logit model, the objective is prediction of a dependent variable from a set of independent variables.

The main vehicle of the log-linear models is the oddsratio. TABLE 1 represents a $2 \times 2$ contingency tables. The odds ratio for this table is calculated as $\left(F_{11} / F_{21}\right) /\left(F_{12} / F_{22}\right)$, or equivalently, $\left(F_{11} F_{22} / F_{12} F_{21}\right)$. While an odds-ratio of one indicates no association, this measure takes on any positive value. An odds-ratio smaller than one indicates an inverse relationship between the odds of $F_{11}$ to $F_{21}$ vs. those of $F_{12}$ to $F_{22}$ (i.e., a negative correlation), while an odds-ratio greater than one would mean a positive correlation in the above sense. Assessment of any cross-tabulation through the loglinear models requires the odds-ratios as the basic form of 
variation which needs to be explained. The general loglinear model develops a number of "reconstruction hypotheses! (or models) for the frequencies in a contingency table. Then the model which has the simplest form (to be defined later) and reconstructs the cell frequencies to an acceptable precision (as determined by the chi-square test of significance) is selected as the model for the data. For instance, the "saturated model" (i.e., the model which includes all possible interactions) would always reconstruct the data perfectly. A two-variable version of this model is discussed next with the purpose of demonstrating the essence of the general log-linear model.

The Saturated Model for a $2 \times 2$ contingency Table

Consider a $2 \times 2$ table with $f_{i j}$ representing the actual frequencies (see TABLE XXXX (a)). The saturated model for this contingency table (i.e., the model which represents all effects, such as the row effects, the column effects, and all interaction effects) would propose a set of expected cell frequencies, $F_{i j}$ 's, (see Table XXXX (b)) exactly the same as the actual cell frequencies, $F_{i j}$. The reconstruction hypothesis is then stated as:

$$
F_{i j}=y^{*} y_{i}{ }^{A_{*} y_{j}}{ }^{B_{\star y}} y_{i j}{ }^{A B}
$$

where;

$$
\begin{aligned}
& y=\left(F_{11} \cdot F_{12} \cdot F_{21} \cdot F_{22}\right)^{1 / 4} \\
& Y_{1}^{A}=\left(Y_{2}{ }^{A}\right)^{-1}=\left(F_{11} \cdot F_{12}\right)^{1 / 2} / Y
\end{aligned}
$$




$$
\begin{aligned}
Y_{1}{ }^{B}=\left(Y_{2}{ }^{B}\right)^{-1} & =\left(F_{11} \cdot F_{21}\right)^{1 / 2} / Y \\
Y_{11}{ }^{A B}=Y_{22}{ }^{A B} & =\left(Y_{12}{ }^{A B}\right)^{-1}=\left(Y_{21}{ }^{A B}\right)^{-1} \\
& =\left(F_{11} \cdot F_{22} / F_{12} \cdot F_{21}\right)^{1 / 4}
\end{aligned}
$$

TABIE XXXX

ACTUAL AND EXPECTED CELL FREQUENCIES FOR A $2 \times 2$ CONTINGENCY TABIE

a) ACTUAL CEIL FREQUENCIES:

\begin{tabular}{|l|l|}
\multicolumn{2}{c}{$Y$} \\
\hline$f_{11}$ & $f_{12}$ \\
\hline$f_{21}$ & $f_{22}$ \\
\hline
\end{tabular}

b) EXPECTED CELI FREQUENCIES:

$\mathbf{Y}$

$x \quad$\begin{tabular}{|l|l|}
\hline$F_{11}$ & $F_{12}$ \\
\hline$F_{21}$ & $F_{22}$ \\
\hline
\end{tabular}

C) A NUMERICAI EXAMPIE:

$$
\mathbf{Y}
$$

$\mathbf{x}$

\begin{tabular}{|l|c|}
\hline 50 & 80 \\
\hline 20 & 100 \\
\hline
\end{tabular}

A numerical example of this model, based on Table $\mathrm{XXXX} .(c)$, is presented below:

$$
\begin{aligned}
y & =53.183, \\
y_{1}{ }^{A} & =1.189, \quad y_{2}{ }^{A}=0.841,
\end{aligned}
$$




$$
\begin{aligned}
Y_{1}{ }^{B} & =0.595, Y_{2}{ }^{B}=1.681 \\
Y_{11}{ }^{A B} & =1.330, Y_{12}{ }^{A B}=0.752, \\
Y_{21}{ }^{A B} & =0.752, Y_{22}{ }^{A B}=1.330
\end{aligned}
$$

Then:

$$
\begin{aligned}
& F_{11}=y^{*} y_{1}{ }^{A}{ }_{Y}{ }_{1}{ }^{B} y_{11}{ }^{A B}=50 \\
& F_{12}=y^{*} y_{1}{ }^{A} y_{2}{ }^{B} * y_{12}{ }^{A B}=80 \\
& F_{21}=Y^{*} Y_{2}{ }^{A} * Y_{1}{ }^{B} y_{21}{ }^{A B}=20 \\
& F_{22}=y^{*} y_{2}{ }^{A} * y_{2}{ }^{B} * y_{22}{ }^{A B}=100
\end{aligned}
$$

It can be shown that $Y, Y_{I}{ }^{A}, Y_{1}{ }^{B}$, and $Y_{I I}{ }^{A B}$ are all based on the odds-ratio. For example, the row-effects odds ratio is stated as:

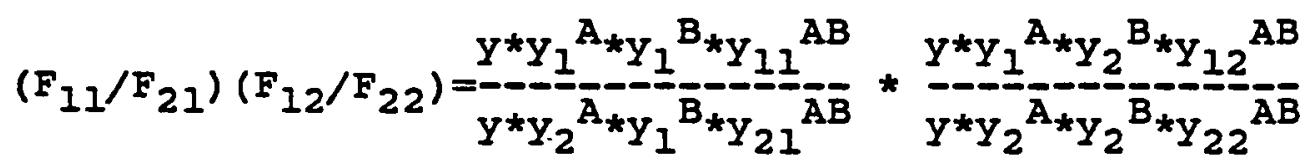

$$
\begin{aligned}
& =\left(Y_{1}{ }^{A} / Y_{2}{ }^{A}\right)^{2}=\left(Y_{1}{ }^{A}\right)^{4}=\left(1 / Y_{2}{ }^{A}\right)^{4}
\end{aligned}
$$

therefore;

$$
\begin{aligned}
Y_{1}^{A}=1 / Y_{2}^{A} & =\left(F_{11} F_{12} / F_{21} F_{22}\right)^{I / 4} \cdot\left(F_{11} F_{12} / F_{11} F_{11}\right)^{1 / 4} \\
& =\left(F_{11} F_{12}\right)^{1 / 2} / F_{11} F_{12} F_{21} F_{22}=\left(F_{11} F_{12}\right)^{1 / 2} / Y
\end{aligned}
$$

\section{other Models}

The saturated models assumes that all effects (i.e., column, row, effects and joint two-way, three-way, etc. interaction effects) are present in a model. It is, 
however, conceivable to eliminate some of these effects by setting them equal to one $(\mathrm{e} . \mathrm{g} \cdot$, in the case of interactions, setting $y_{11} A B=1$ would follow that $y_{i j} A B=1$ for all $i$ and $j$ which means $A$ and $B$ are independent). Then, this model would be acceptable if the generated expected frequencies (i.e., Fij's) are statistically the same as the actual frequencies (i.e., using the chi-square test for independence). Thus for the two dimensional contingency table (TABLE 2) the following models can be hypothesized:

$$
\begin{aligned}
& F_{i j}=y^{*} y_{i}{ }^{A} y_{j} B_{* y_{i j}}^{A B} \\
& F_{i j}=y^{*} y_{i}{ }^{A} y_{j} B \\
& F_{i j}=y^{*} Y_{i}^{A} \\
& F_{i j}=y^{*} y_{j}^{B} \\
& F_{i j}=y
\end{aligned}
$$

If these models are converted to their log-linear form, their interpretation becomes easier. For instance, taking the natural logarithm of all terms in the saturated model, $F_{i j}=y^{*} y_{i}{ }^{A}{ }_{y_{j}}{ }^{B}{ }_{*} y_{i j}{ }^{A B}$, will yield:

(29) $\log (F i j)=\log y+\log y_{i}^{A}+\log y_{j}^{B}+\log Y_{i j}{ }^{A B}$ which can be re-written as:

(30) $\log F_{i j}=u+u_{i}^{A}+u_{j}^{B}+u_{i j} A B$

The above expression can be given a regression-like interpretation with $u$ as the intercept, $u_{i}{ }^{A}$ as the effect of 
variable $A$ alone, $u_{j}{ }^{B}$ as the effect of variable $B$ alone, and $u_{i j} A B$ as the joint effect of variables $A$ and $B$.

\section{Hierarchical Log-Iinear Models}

A very common version of the general log-linear model known as the hierarchical structure assumes that each model having a specific interaction term (e.g., $u_{i j}{ }^{A B}$ ) includes all of the lower level terms $\left(e . g ., u, u_{i}{ }^{A}, u_{j}{ }^{B}\right.$ ). In this type of structure, the notation can be simplified. For example, the saturated two-variable model can be represented by $\{A B\}$. Similarly a multivariate model such as:

$$
\begin{aligned}
& \log F_{i j}=u+u_{i}{ }^{A}+u_{j} B+u_{k} C+u_{1} D \\
& +u_{i j} A B+u_{i k} A C+u_{i l} A D+u_{j k} B C+u_{j l} B D \\
& +u_{k l} C D+u_{i j k} A B C+u_{i j l} A B D+u_{j k l} B C D
\end{aligned}
$$

can be represented by $\{A B C\}\{A B D\}\{B C D\}$. In addition to simplifying the representation of a certain model, the above notation designates the subtables that need to be analyzed instead of the original contingency table.

\section{Generating the Expected Frequencies}

For a $2 \times 2$ table such TABLE 2, the expected frequencies are easily calculated. However, for more complicated tables, the log-linear models fall into two categories: Those with explicit formulae for generating expected frequencies (the direct or closed formed) and those without 
such formulae (the indirect or open formed) . (Reynolds, 1977).

In order to detect whether a model is closed or open formed, Reynolds (1977) offers a five step procedure (and also both Broekstra, 1981, and Krippendorff, 1981, offer a similar procedure when encountering the same problem in the information theoretic context). Then, to generate the expected frequencies for the open formed models an iterative method is selected --e.g., the Newton-Raphson or the DemingStephen algorithms (Knoke and Burke, 1980). After the expected frequencies are calculated for the proposed models, they are tested for independence using chi-square. The model with the lowest chi-square is then selected as the best model, as it reconstructs the data most closely. 
APPENDIX II

ENTROPY AND INFORMATION THEORETIC CONCEPTS

As is well known, the foundations of mathematical information theory were laid down by Shannon (Shannon and Weaver 1949) during his work on the optimization of communication systems. Since then, this concept has made its way into the literature of Economics (Theil 1967), Psychology (Attneave 1959, Miller 1953, Garner 1962), Marketing (Herniter 1973, 1974 Bass 1976), Accounting (Lev 1968, 1970), Finance (Philipatos and Gressis 1975), and other disciplines.

It has been well established that the amount of potential information obtained from observing any state $\mathrm{x}$ of some phenomenon $x$ can be measured by $-\log P_{x} ;$ where $P_{X}$ is the probability of $\mathrm{x}$ being in state $\mathrm{x}$. In general, however, all states of a variable do not have equal prior probabilities. Therefore, the amount of potential information to be gained from state $x$ has to be weighted by the probability of its occurrence. If successive states are chosen independently, with fixed probabilities $P_{x}$, then a 
convenient measure of the average amount of information gained with the knowledge of each state is:

$$
H(X)=-\sum_{X=1}^{N} P_{X} \log P_{x} \cdot
$$

Equation (32) is known as the amount of information or the entropy of the source, because of the formal similarity of (32) with the thermodynamic definition of entropy (Shannon and weaver 1949). The choice of a logarithmic base for Equation (32) is arbitrary. Commonly, in information theory, logarithms to base two are used, and information units are then measured in BInary digiTs (or BITs). Equation (32) can be extended to include both the multivariate case and the case with continuous distribution. In the multivariate case, (32) can be rewritten as:

$$
H(A, B, \ldots, X)=-\sum_{a \varepsilon A} \sum_{b \varepsilon B} \ldots \sum_{x \in X} P_{a b} \ldots x \log P_{a b} \ldots x^{\prime}
$$

if the variable is continuous, then (32) is:

$$
H(f(x))=-\int f(x) \log f(x) d x .
$$

The entropy of a continuous variable behaves in much the same way as does the entropy of a discrete variable. The entropy $H(X)$ can be interpreted as a measure of the variability (scatter, spread, uncertainty, behavioral 
The entropy $\mathrm{H}(\mathrm{X})$ can be interpreted as a measure of the variability (scatter, spread, uncertainty, behavioral freedom) of the variable. This measurement is a "unique, unambiguous" criterion based on the intuitive notion that a broad distribution represents more uncertainty than does a narrow, sharply peaked distribution (Herniter 1973). It is a non-negative quantity applicable to metric and non-metric variables. A zero value for $\mathrm{H}(\mathrm{X})$ indicates the availability of only one state for $x$ (i.e., $\log _{2} 1=0$ ): thus, $x$ would have no "choice" or "behavioral alternative." Maximum freedom of choice is obtained when $H(X)$ is calculated from a uniform distribution (i.e., when all the states are equally probable), in which case $H(X)=\log _{2} N ; N=$ the number of possible states for $X$.

In a similar vein, a system with two variables, $x$ and $Y$, can be conceived as having total (joint) behavioral freedom, indicated by $H(X, Y)$. Intuition suggests that this quantity, $H(X, Y)$, is maximum if variables $X$ and $Y$ behave independently. In fact, this maximum possible value; denoted as $H_{\max }(X, Y)$, can be shown to be equal to the sum of the individual freedoms for variables $X$ and $Y$ (Broekstra 1981). That is:

$$
H_{\max }(X, Y)=H(X)+H(Y)
$$


On the other hand, if the two variables are mutually associated, their joint behavioral freedom is reduced, as they impose behavioral constraints upon one another.

We will next discuss what is known as conditional entropy and "transmission." For a system consisting of two variables $X$ and $Y$, say $A=\{X, Y\}$, the variability of $A$, which is not accounted for in some subset of $A, e . g .,\{Y\}$, is measured by the conditional entropy defined as (Broekstra 1981):

$$
H_{Y}(X)=H(X, Y)-H(Y)
$$

This quantity, $\mathrm{H}_{Y}(\mathrm{X})$, is a measure of one's average uncertainty as to the state of $x$, given the knowledge about the state of $Y$. Its value ranges from 0 , when $Y$ completely determines $x$, to $H(x)$ for complete independence between $x$ and $Y$. One may also note the well known fact that:

$$
H_{Y}(X) \leqslant H(X)
$$

to which an intuitive meaning may be attached. That is, knowledge about the state of $Y$ will, in general, not increase the uncertainty existing about the state of $x$. In expression (37), equality holds if and onl $Y$ if $X$ and $Y$ are independent, while $H_{Y}(X)$ vanishes if and only if the state of $\mathrm{X}$ is completely determined once the state of $\mathrm{Y}$ is known. As pointed out by Krippendorff (1979), whenever the 
probabilities of joint events are the product of the probabilities of individual events, entropies are additive, and the lack of additivity signifies interdependence. Thus, if $X$ and $Y$ are independent, $H(X, Y)=H(X)+H(Y)$. The difference between $H(X, Y)$ (i.e., the actual entropy) and $H(X)+H(Y)$ (the maximal entropy) is then a measure of interdependence.

In developing information theory into a multivariable calculus, Garner and McGill (1956) and Ashby (1964) have generalized Shannon's (Shannon and Weaver 1949) binary notion of "association" into a multivariate expression known as "transmission" or amount of "transmitted information," denoted by $T$. For two variables $X$ and $Y$, for instance, the degree of association between $X$ and $Y$, denoted by $T(X: Y)$, may be expressed as the difference between the marginal and the joint variability. That is, transmission $T(X: Y)$ is given by:

$$
\begin{aligned}
T(X: Y) & =H_{m a X}(X, Y)-H(X, Y) \\
& =H(X)+H(Y)-H(X, Y) \\
& =H(X)-H_{Y}(X) .
\end{aligned}
$$

The above expressions measure the amount of statistical dependence (or constraint or relatedness) of a pair of variables $X$ and $Y$. Transmission is symetric, i.e., $T(X: Y)$ $=T(Y: X)$, and non-negative. It is equal to zero if and only if the variables are statistically independent, and equal to the smaller of $\{H(X), H(Y)\}$ if and only if the knowledge of 
the state of one variable completely reveals the state of the other variable. (Broekstra 1981).

owing to this behavior of the transmissions with respect to its range, $O \leqslant T(X: Y) \leqslant \operatorname{Min}\{H(X), H(Y)\}$, its numerical value may be thought of as expressing the strength of the interdependence between $X$ and $Y$. This measure is completely decomposable, which can be partitioned into variability between and variability within sets of variables, allowing for ANOVA-like analysis of variables (Garner and McGill 1956, Horowitz and Horowitz 1976). For the multivariate case, expression (38) can easily be shown as (Garner and McGill 1956, Horowitz and Horowitz 1976):

$$
T(A: B: C: \ldots: X)=H(A)+H(B)+H(C)+\ldots+H(X)-H(A, B, C, \ldots, X)
$$

Equation (39) represents the total amount of interdependence or constraint existing within the system as a whole. If this quantity is equal to zero, the variables are thought to be jointly independent. The, maximum for equation (39) is defined as $H(i)-\max \{H(i)\} ; i=A, B, C, \ldots, X$.

Another important type of transmission function remains to be discussed. That is the information transmitted from one variable to another where a third is already known (or held constant). For instance, information shared by $A$ and $B$ when $X$ is held constant is denoted by 
$\mathrm{T}_{\mathrm{X}}(\mathrm{A}: \mathrm{B})$. This measure can be calculated as (Garner and MCGill 1956, Ashby 1964, Krippendorff 1979):

$$
\mathrm{T}_{\mathrm{X}}(\mathrm{A}: \mathrm{B})=\mathrm{T}(\mathrm{A}: \mathrm{B}: \mathrm{X})-\mathrm{T}(\mathrm{A}: \mathrm{X})-\mathrm{T}(\mathrm{B}: \mathrm{X}) ;
$$

where $\mathbf{T}_{X}(\mathrm{~A}: \mathrm{B})$ is the conditional transmissions which measures statistical dependence between the pair of variables $A$ and $B$, while a third variable, $x$, is held constant. In general (Garner and McGill 1956, Ashby 1964, Krippendorff 1979):

$$
\text { (41) } T_{X}(A: B: C: \ldots)=T(A: B: \ldots: X)-T(A: X)-T(B: X)-T(C: X)-\ldots
$$

Also, the uncertainty of a variable in a multivariate system can be decomposed into the portion explained by other variables (i.e., transmissions between that variable and the remaining variables), and the portion not represented by any other variable (i.e., the uncertainty specific to the variable under consideration) (Abrahamse and van Bueren 1980, Krippendorff 1986). For instance, in a four variable system $\{W X Y Z$, the following breakdown of the uncertainty for the variable $w$ may result:

$$
\mathrm{H}(\mathrm{W})=\mathrm{T}(\mathrm{W}: \mathrm{X})+\mathrm{T}_{\mathrm{X}}(\mathrm{W}: \mathrm{Y})+\mathrm{T}_{\mathrm{XY}}(\mathrm{W}: \mathrm{Z})+\mathrm{H}_{\mathrm{XYZ}}(\mathrm{W})
$$

In Equation (42), $T_{X}(W: Y)$ and $T_{X Y}(W: Z)$ are conditional transmissions, which have been defined above, and the conditional entropy, $\mathrm{H}_{\mathrm{XYZ}}(\mathrm{W})$, is the amount of uncertainty remaining in variable $W$ when the states of $X, Y$, and $z$ are known. 
Equation (42) is useful in prioritizing the contributions to the reduction of the uncertainty in a criterion variable (e.g., w) by a number of explanatory variables (e.g., $X, Y$, and $z$ ) through sequentially selecting the highest contributors. For instance, in choosing the highest contributor to the reduction of uncertainty in $w$, $\operatorname{MAX}(T(W: i), i=x, y$, or $z\}$ is selected. When selecting the second highest contributor, $\operatorname{MAX}\left\{T_{i}(W: j), j=X, Y\right.$, or $Z$, $j=i\}$ is selected, and so on. This operation first identifies the highest contributor, then, assuming that this variable is given, identifies the next highest contributor, thus eliminating the overlap between the two explanatory variables in reducing the uncertainty in the criterion variable. Therefore, each variable's incremental effect on uncertainty reduction of the criterion variable is measured in each successive step and the highest contributor is selected.

Another quantity which is useful in assessing different structural models (as will be addressed in detail in the next section) is the amount of "interaction." Interaction is a measure of the uniqueness of the relationship within a set of variables, attributed only to the n-way effects among them. For instance, the interaction between three variables, denoted by $Q(A, B, C)$, has a unique value, which may be defined in one of the following equivalent ways (Broekstra 1981): 
(43)

$$
\begin{aligned}
Q(A, B, C) & =T_{A}(B: C)-T(B: C) \\
& =T_{B}(A: C)-T(A: C) \\
& =T_{C}(A: B)-T(A: B)
\end{aligned}
$$

Note that interaction term $Q$ can be either positive or negative since transmissions and conditional transmissions are non-negative quantities. 
Northwest Electric Power Planning and Conservation Act of 1980 (Public Law 96-501).

\title{
PACIFIC NORTHWEST RESIDENTIAL ENERGY CONSUMPTION SURVEY
}

\author{
Spring 1983
}

CODE BOOK

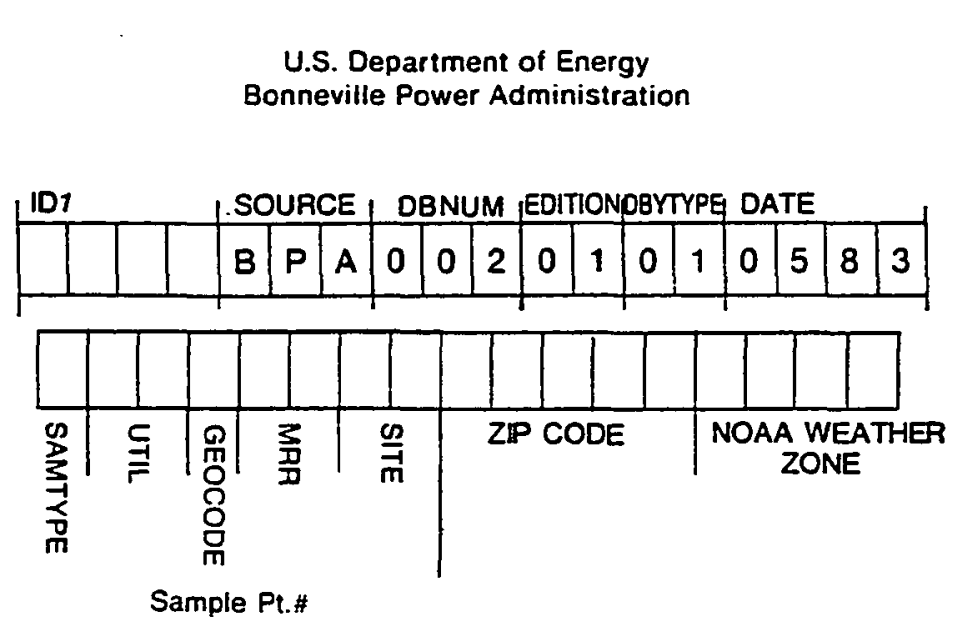


ERRATA

Due to an oversight, the SPSS Data Definition (DD) Files for the NOAA Weather Data files were improperly specified. The DD File contained in the computer tape is formatted to read only one record per case. However, the actual data contains four records per case, each record containing the data for one of the four sets of heating degree days (HDD) and cooling degree days (CDD) base temperatures.

Please note that to read the data properly, the user must alter the format statement and the list of variables. Following is a copy of the DD file contained on the tape:

ID,CZ,HHDBASE, CDDBASE, HDD001 TO HDD020,CDD001 TO CDD020

$(4 F 5.0,40 F 8.0)$

This DD is used for both the electricity billing weather data and the gas billing weather data.

For electricity billing weather data, the DD file should read as follows:

EID1,ECD1, EHDD1B ,ECDD1B,EHDD1001 to EHDD1020,ECDD1001 to ECDD1020,

EID2,ECD2, EHDD2B ,ECDD2B ,EHDD2001 to EHDD2020,ECDD2001 to ECDD2020,

EID3, ECD3, EHDD3B, ECDD3B, EHDD3001 to EHDD3020,ECDD3001 to ECDD3020,

EID4,ECD4,EHDD4B, ECDD4B, EHDD4001 to EHDD4020,ECDD4001 to ECDD4020,

$(4 F 5.0,40 F 8.0 / 4 F 5.0,40 F 8.0 / 4 F 5.0,40 F 8.0 / 4 F 5.0,40 F 8.0)$

For the gas billing weather data, the DD file should read as follows:

GID1,GCD1, GHDD1B, GCDD1B, GHDD1001 to GHDD1020,GCDD1001 to GCDD1020, GID2, GCD2, GKDD2B, GCDD2B , GHDD2001 to GHDD2020, GCDD2001 to GCDD2020, GID3, GCD3, GHDD3B, GCDD3B ,GHDD3001 to GHDD3020,GCDD3001 to GCDD3020, GID4,GCD4, GHDD4B,GCDD4B, GHDD4001 to GHDD4020,GCDD4001 to GCDD4020, $(4 F 5.0,40 F 8.0 / 4 F 5.0,40 F 8.0 / 4 F 5.0,40 F 8.0 / 4 F 5.0,40 F 8.0)$ 
1a. Is there any apartment, guest house, or other type of separate living quarters served by the same electric meter you are connected with?

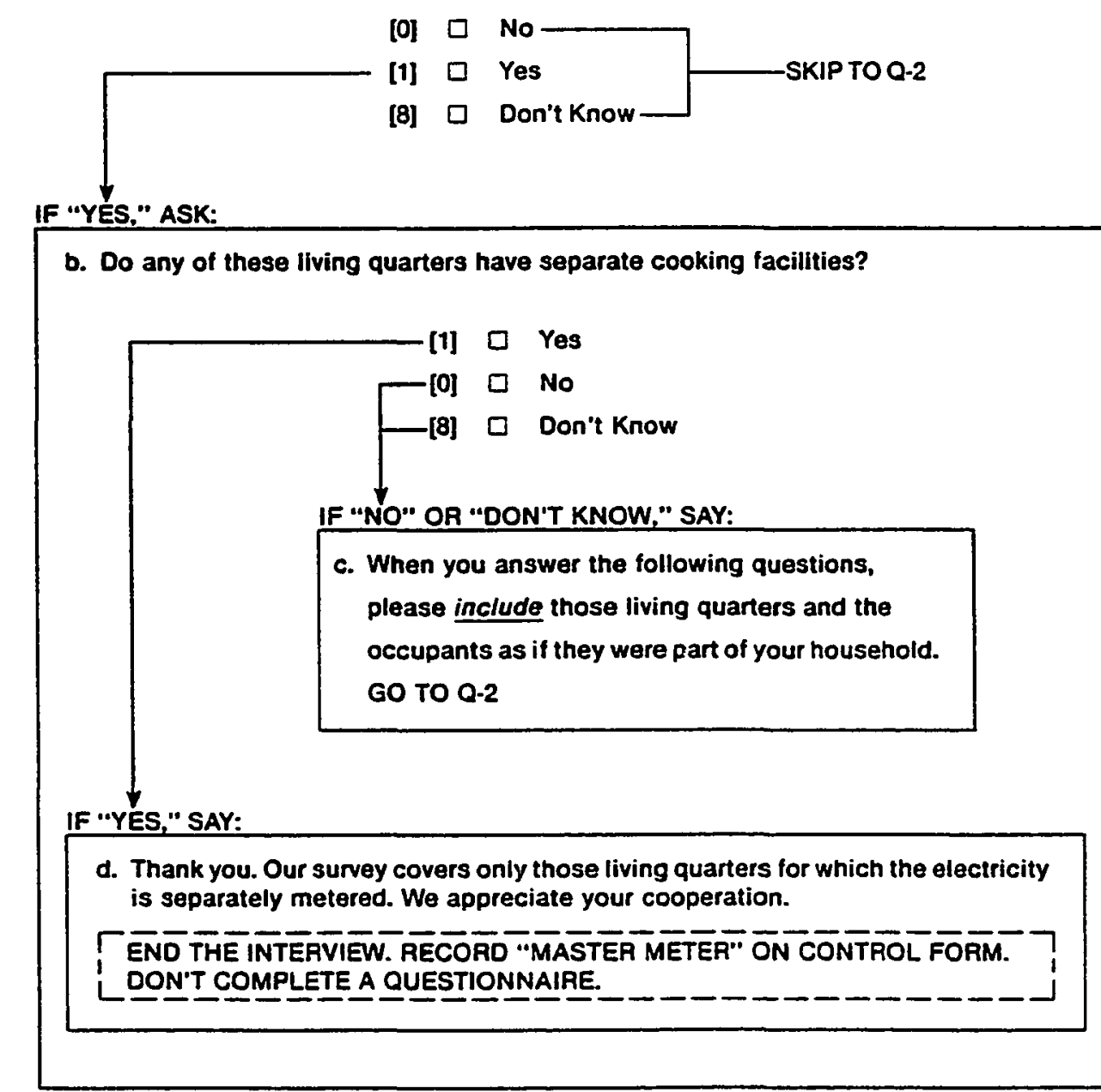




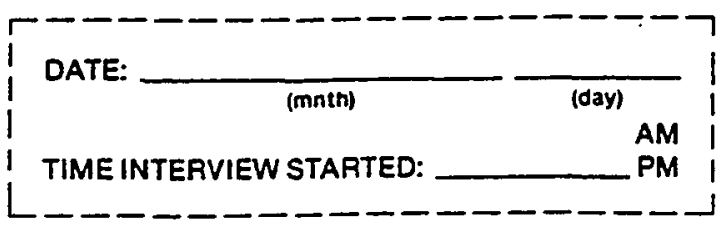

2. Record type of dwelling. If in doubt, verify with respondent, using Exhibit \#2.

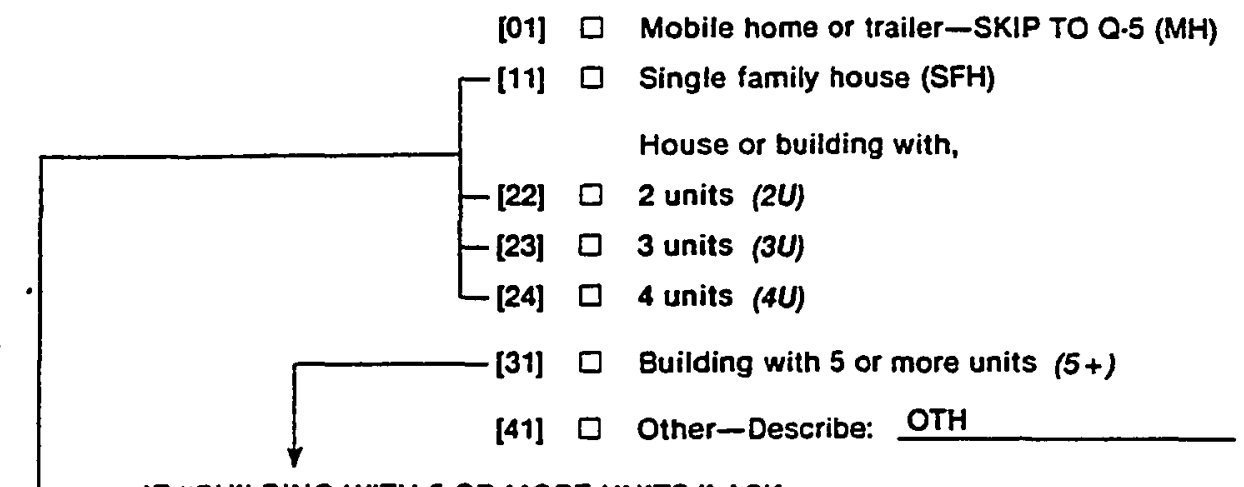

IF "BUILDING WITH 5 OR MORE UNITS," ASK:

3. How many units does this building contain? _(Units)

006

$\checkmark$ SKIP TOQ Q.5

IF "SINGLE FAMILY" OR "BUILDING WITH 2 TO 4 UNITS," ASK:

4. Record the following. If in doubt, verify with respondent. House or building is...
[1] Detached
[2] $\square$ Attached on one side
[3] $\square$ Attached on two sides
[7] $\square$ Not applicable

007

5. Do you or members of your household own your home or do you rent?

[1] $\square$ Own or Buying-SKIP TO Q-7

[2] $\square$ Rent

[3] $\square$ Occupied without payment of rent

008

IF "RENT" OR "OCCUPIED WITHOUT RENT," ASK:

6. Which of the following are furnished as part of the house (apartment)? READ LIST

$\begin{array}{lccc} & \begin{array}{c}\text { No } \\ \text { [0] }\end{array} & \text { Yes } & \text { NIA } \\ & {[1]} & {[]} \\ \text { Refrigerator ....... } & \square & \square & \square \\ \text { Stove top/burners . } & \square & \square & \square \\ \text { Oven ........... } & \square & \square & \square \\ \text { Clothes Washer .... } & \square & \square & \square \\ \text { Clothes Dryer ...... } & \square & \square & \square \\ \text { Olshwasher........ } & \square & \square & \square\end{array}$


7. In what year did you first move into this house (apartment)?

Actual year

IF "DON'T KNOW," ASK:

$$
\text { [998] } \square \text { Don't Know }
$$

8. Would you mind reviewing Exhibit \#8 and indicating the category which you think best describes your estimate of the time when you first moved into this house (apartment)?
[01] $\square$ (a) Before 1940
[06] $\square$ (f) $1975-1978$
[02] $\square \quad$ (b) $1940-1949$
[07] $\square \quad$ (g) $1979-1981$
[03] $\square \quad$ (c) $1950-1959$
[08] $\square \quad$ (h) 1982-1983
[04] $\square$ (d) $1960-1969$
[98] $\square$ Don't Know
[05] $\square \quad$ (e) $1970-1974$
[97] $\square \quad$ Not Applicable

9. Did any of the current members of your household move into this house (apartment) before you moved in?

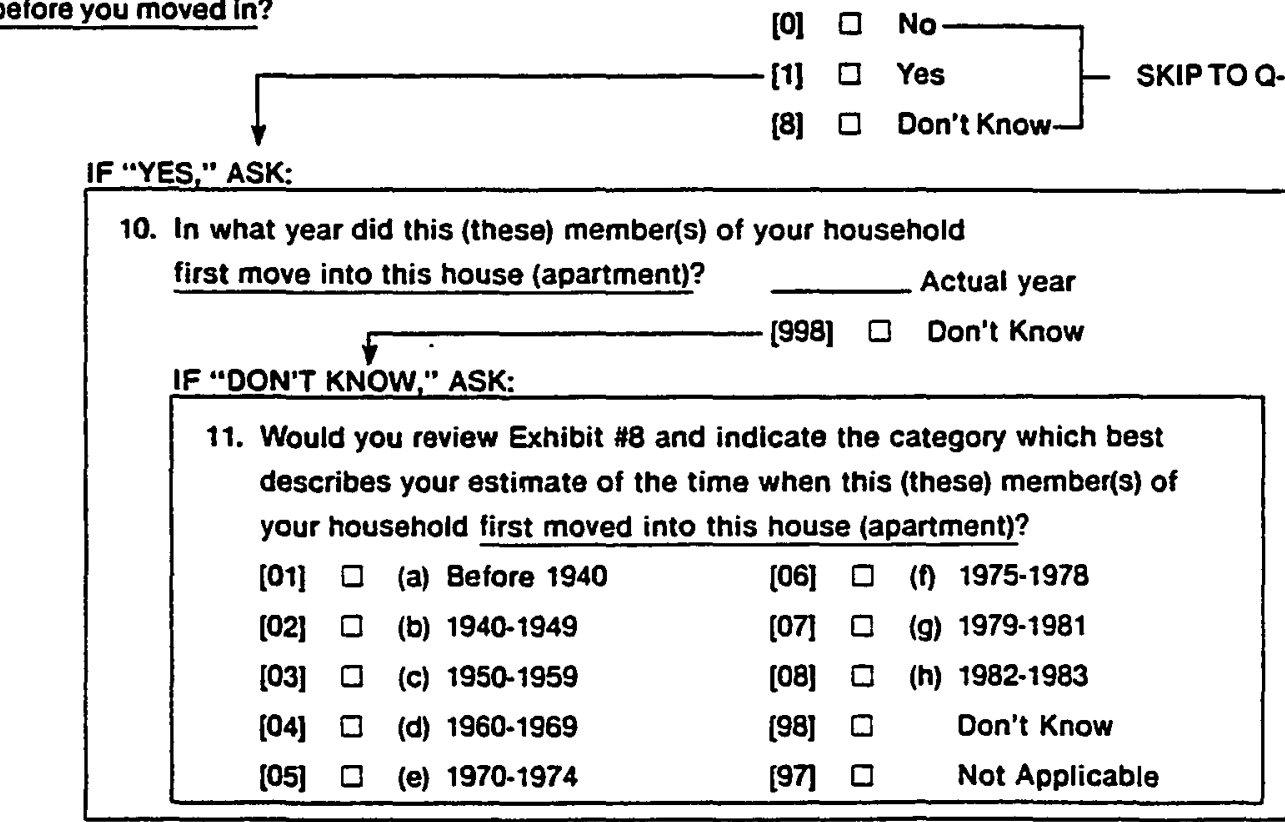

IF “1981," "1982" OR "1983," ASK:

12. In which year and month did you (this/these member[s]) first move in? (Circle the applicable months.)

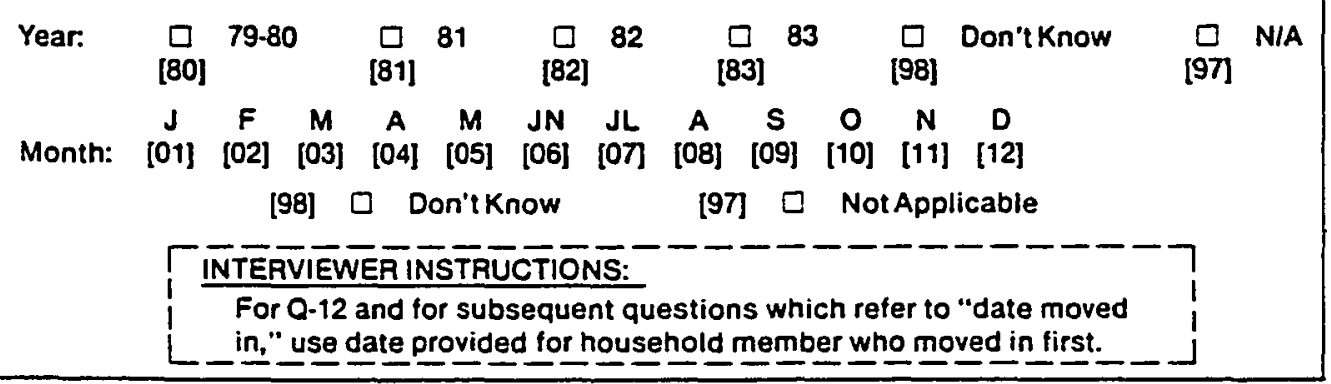

BDA ISM PAGE A 
13. Was this house (building) newly constructed when you (member[s] of your household) first moved in?

$$
\begin{array}{lll}
{[0]} & \square & \text { No } \\
{[1]} & \square & \text { Yes } \\
{[8]} & \square & \text { Don't Know/Don't Remember }
\end{array}
$$

14. In what year was this house (building) constructed?

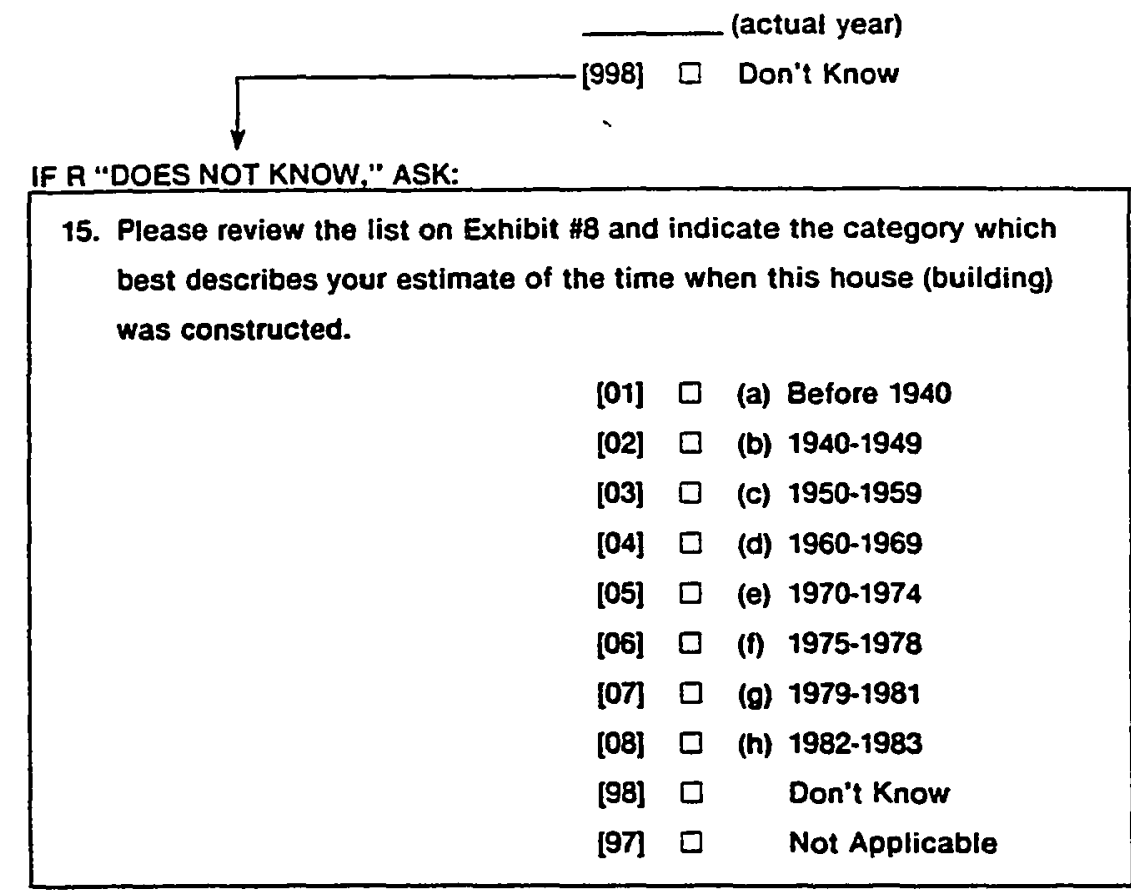

16. Has this house (building) been substantially renovated or remodeled since you (member[s] of your household) tirst moved in? That is, has work costing at least $\$ 5,000$ been done on the house, and including such things as the addition of a new room; the rearrangement of internal walls; the installation of new windows or complete overhaul of existing windows? Redecoration of the house is not included-for example new paint or wallpaper, new carpets or floors, or new cabinets.

$$
\begin{aligned}
& \text { [0] } \square \text { No } \\
& \text { [1] } \square \text { Yes } \\
& \text { [8] } \square \text { Don't Know }
\end{aligned}
$$


17. Exhibit \#17 contains a list of different types of rooms which you may have in your home. Please tell me how many of each of these types of rooms there are in your home.

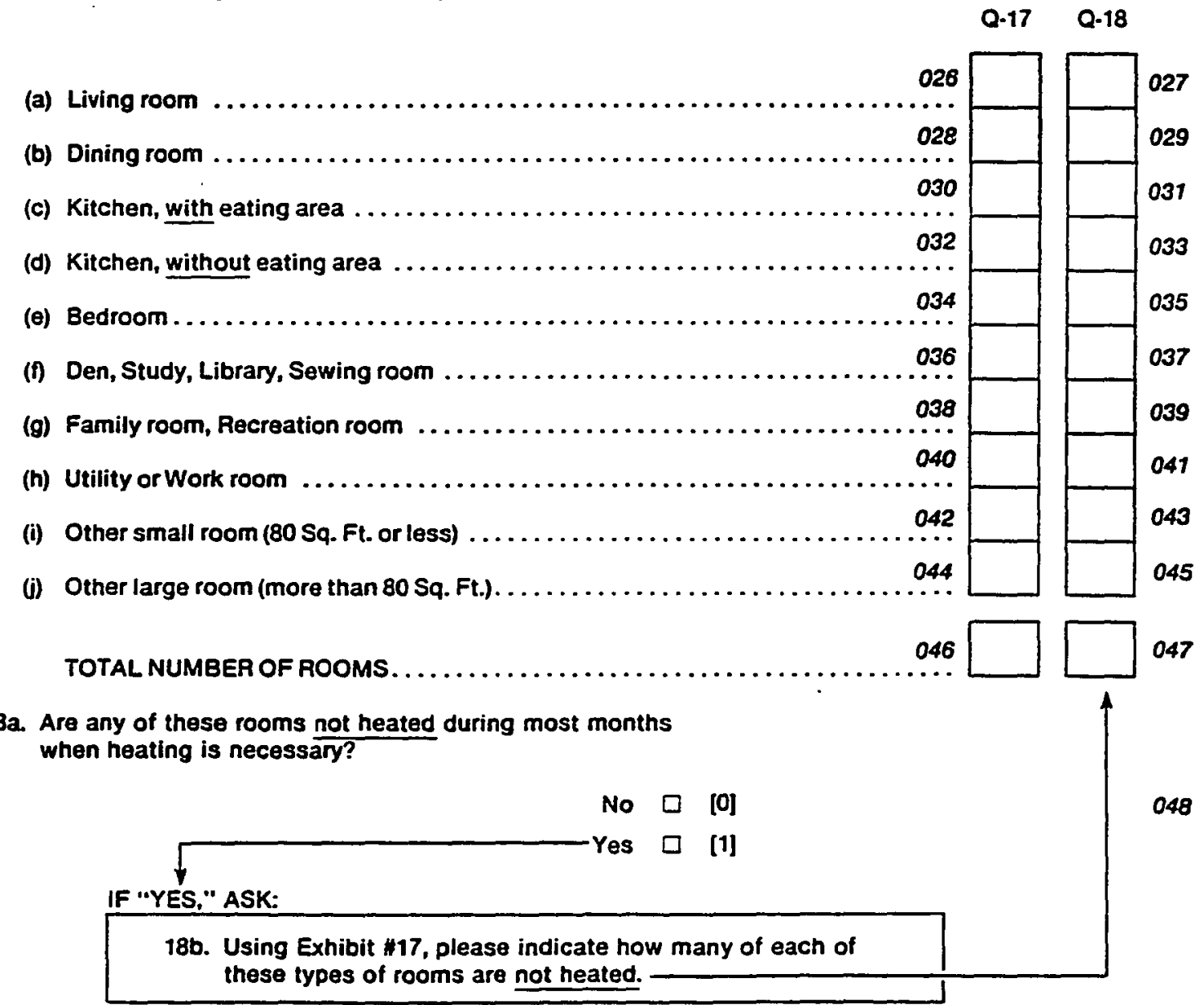

INTERVIEWER INSTRUCTIONS:
Q-17: Do not count bathrooms, entryways, hallways, unheated porches,
unfinished attics, or unfinished basements.
Count living room/dining room combinations (no partitioning wall
between) as a single room and code as "Living room." Probe to
determine whether there is an attic and, if so, whether it is heated.
Q-18: Count a room as not heated if the door is closed and the heat is
shut off or vents are closed. The room may still be used (for sleeping
for example), if the door is closed and the heat is off while the room
is in use.
If there is an attic, probe to determine whether it is heated.


19. Please review the list on Exhibit $\$ 19$ and indicate which of the statements best describes how you feel about the energy efficiency of your home?
[1] $\square$ (a) The home is about as energy efficient as it can be
[2] (b) A little improvement can be made
[3] $\square$ (c) Moderate improvement can be made
[4] $\square$ (d) A lot of improvement can be made
[8] $\square$ Don't Know
[9] $\square$ Refused

20. I am going to read you a series of items. Each item refers to an issue which some people may consider to be a problem in their state. We would like to know your thoughts about how serious each of these issues is today in your state. Exhibit \#20 contains a list of the answers you may choose from. As I read each item, please tell me whether you consider the item to be not serious at all, slightly serious, moderately serious or very serious.

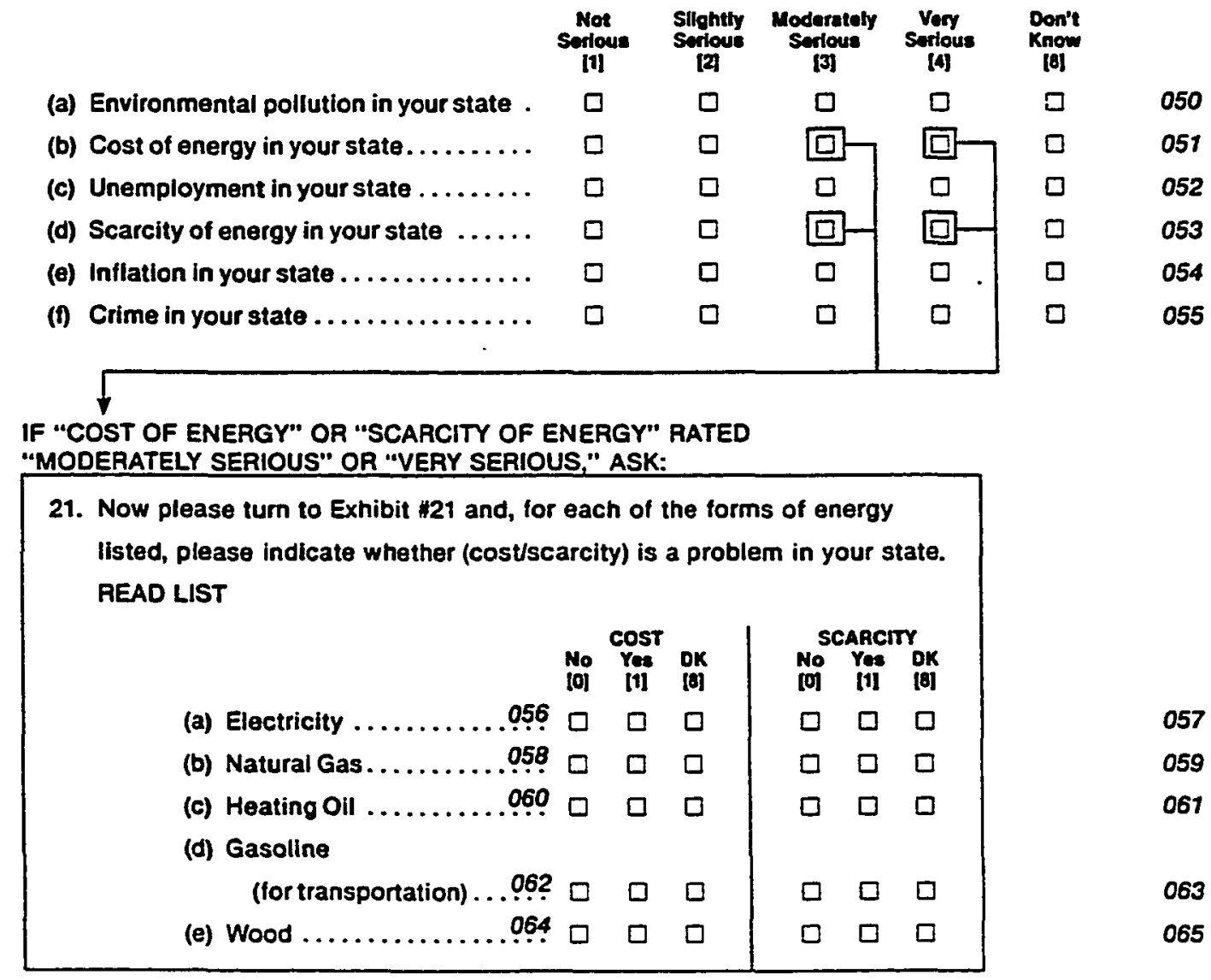


22. If you would please turn to Exhibit \#22 you will find a list of statements. I am going to read each of these statements to you. For each statement, please tell me how strongly you agree or disagree with the statement. At the bottom of Exhibit \#22 there is a list of the answers you may choose from. Let us begin with the first statement.

(a) In the winter, I find it difficult to be comfortable when the temperature in my home is $68^{\circ} \mathrm{F}$ or less. . . . . . . . . . . . . . .

(b) Reducing the temperature of the water heater from $140^{\circ} \mathrm{F}$ to $120^{\circ} \mathrm{F}$ saves enough energy to make it worth doing. . .

(c) The main reason to conserve energy is to save money. ....................

(d) During the winter, when no one will be home for 2 hours or more, turning the temperature down is worthwhile. .......

(e) It's hard for me to get around to making my home more energy efficient. ........

(f) People have a right to use as much energy as they want and can pay for. ....

(9) The price I first pay for the appliance is more important to me than the energy savings. .......................

(h) To conserve enough energy to make a difference in my bills I would have to change my lifestyle.

$$
\text { Strong }
$$

Strongly
Agrise
$\square$
$\square$

Somowhat Agree

Undeclded

Somowhat Strongly Disagres Disagree

$\square$

$\square$

$\square$

$\square$

066

067

(1)

$\square$

$\square$

$\square$

$\square$

067

$\square$

$\square$

$\underset{\mathfrak{1}}{\square}$

$\square$

068

$\square$

069

$\square$

$\underset{12}{\square}$

$\square$

$\square$

$\underset{9}{\square}$

070

$\square$

$\square$

$\square$

$\square$

$\square$

$\square$

071

[1]

$\underset{12}{\square}$

$\square$

[5]

072

(1)

$\square$

$\square$

$\square$

$\square$

$\square$

$\square_{\mathfrak{I}}$

$\square$

073

23. Please review the list on Exhibit $\$ 23$ and, for each item, indicate whether you have heard or read anything about the item. READ LIST

(a) Federal income tax credit for home weatherization ............... Q74

$$
\text { No } \begin{aligned}
& \text { Q-23 } \\
& \text { Yes }
\end{aligned}
$$

(b) State tax benefit for home weatherization

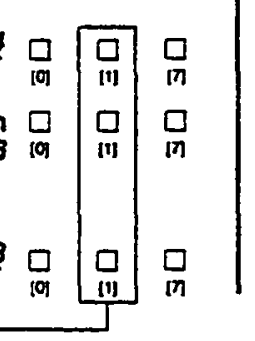

$$
\begin{array}{lll}
\square & \square & \square \\
\square & \square & \square \\
\square & \square & \square
\end{array}
$$

(c) Low or no interest loan from electric or gas utility or fuel oil supplier for home weatherization ............... o7?

\section{FOR EACH "YES," ASK:}

24. Have you made use of ? 
25. To the best of your knowledge, has an energy professional made a physica! inspection of your home, including measurement of insulation levels, and advised you on ways to make your home more energy efficient?

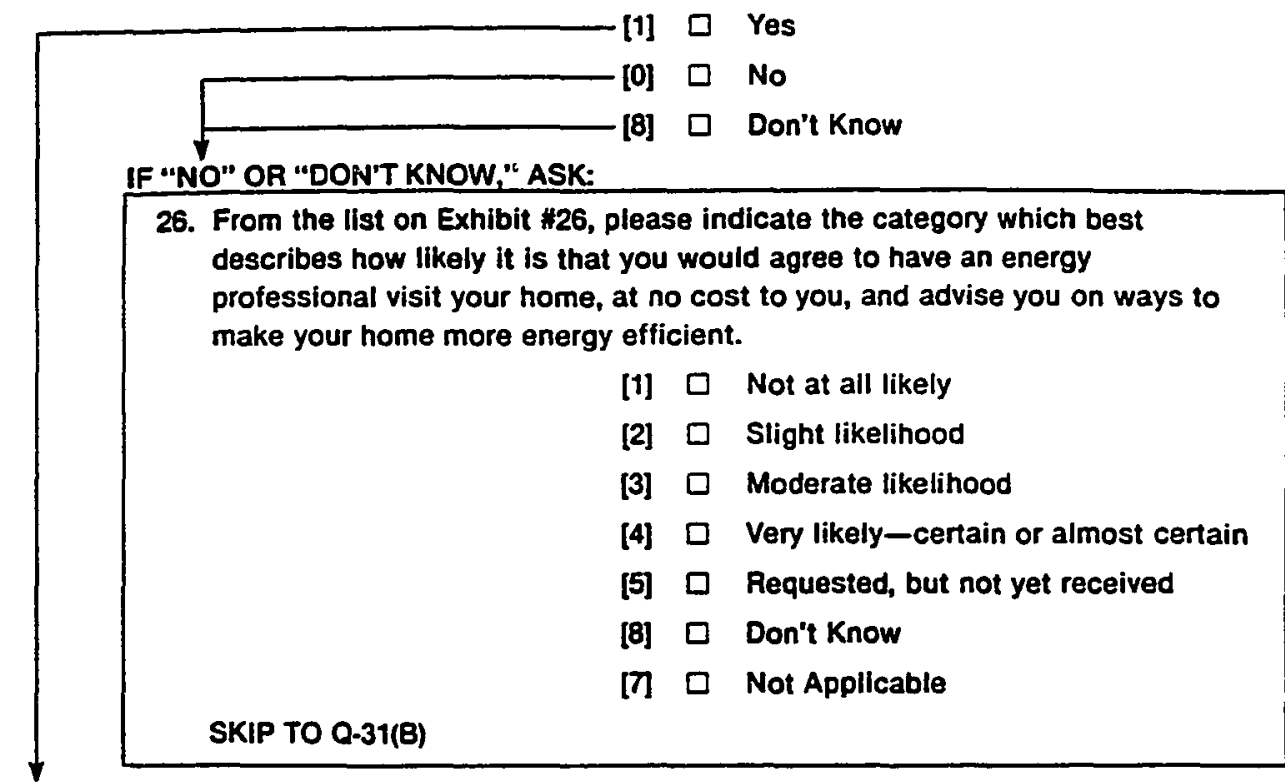

080

IF "YES," ASK:

27. When was your home last inspected by an energy professional? [If month is DK, Code "OO"] Month Year IF AESPONDENT "DOES NOT KNOW," ASK:

[98] $\square$ Don't Know-Month \& Year

[97] $\square$ Not Applicable

28. Would you mind turning to Exhibit \#28 and indicating your best estimate of the time when your home was last inspected by an energy professional?

[1] $\square$ Before 1/1/78

[2] $\square$ Between 1/1/78 and 12/31/79

[3] $\square$ Between 1/1/80 and 12/31/81

[4] $\square$ After $1 / 1 / 82$

084

[8] $\square$ Don't Know

[7] $\square$ Not Applicable

29. Was the inspection conducted by a representative of your electric utility, your gas utility, a heating oil company or by a private contractor?

[1] $\square$ Electric Utility

[2] $\square$ Gas Utility

[3] $\square$ Heating Oil Company

[4] $\square$ Private Contractor

[8] $\square$ Don't Know

(7) $\square$ Not Applicable 
30a. Exhibit \#30/31 contains a list of energy saving measures. Please review the list and, for each measure, indicate whether it was included in the energy professional's recommendations of measures to be taken to improve the energy efficiency of your home. READ EACH ITEM

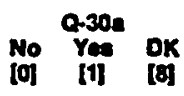

31a. Now, would you please indicate which actions, if any, you had taken before the inspection was completed, but within the last 3 years, that is, since May 1980 ?

IF "NO ENERGY INSPECTION" (Q-25), ASK:

31b. Would you please review the list on Exhibit \#30/31 and indicate which actions you have taken in the last three years - that is, since May 1980? 
32. To the best of your knowledge, which of the categories on Exhibit \#32 best describes the percentage of the outside walls of your home that currently contain insulation?

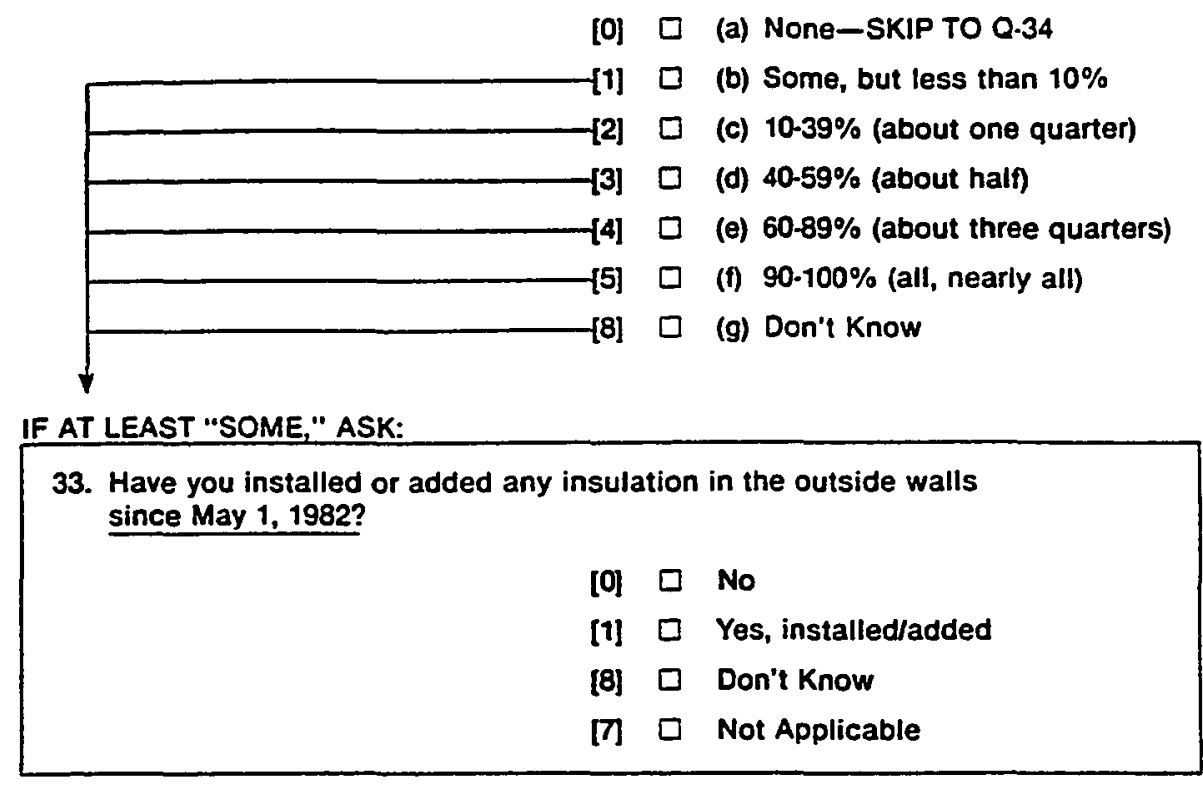

34. To the best of your knowledge, which of the categories on Exhibit \#32 best describes the percentage of the roof, ceiling, or attic of your home that currently contains insulation?

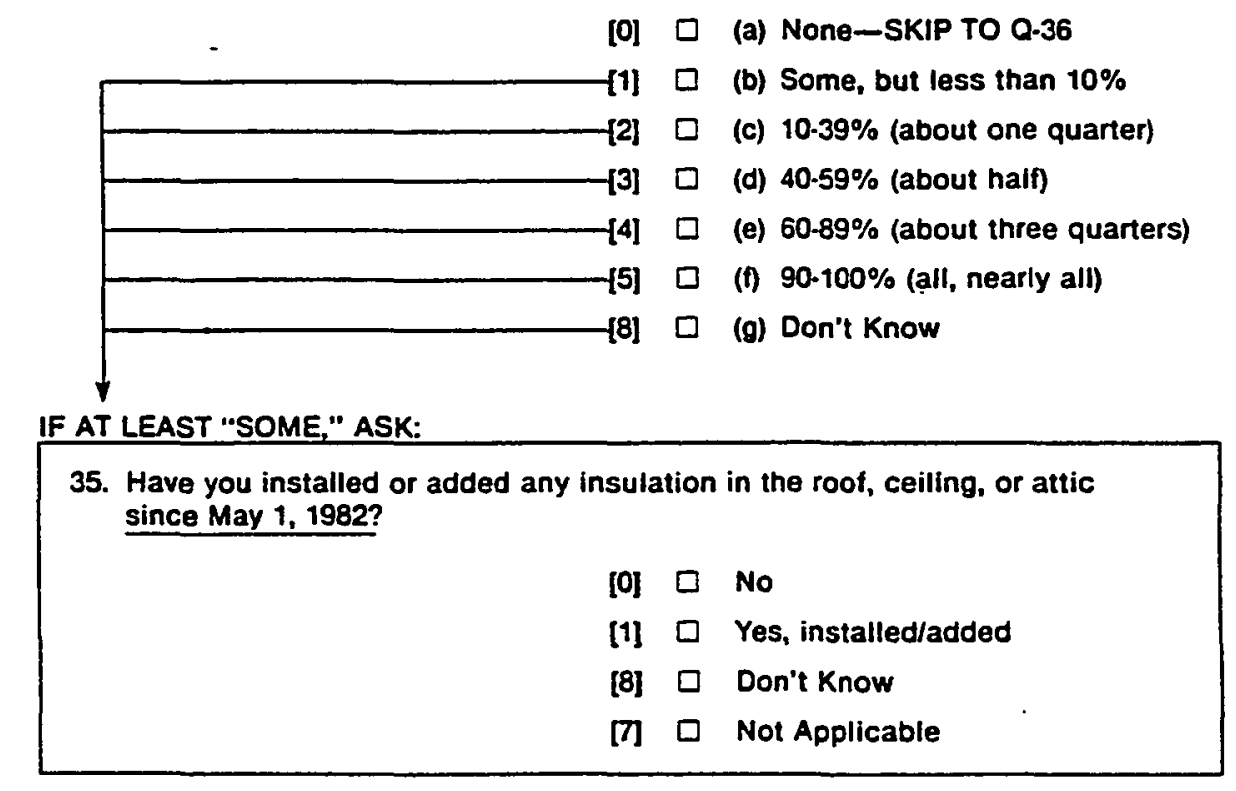


IF "ONE-FAMILY HOME" OR “MOBILE HOME," ASK:

36. Please turn to Exhibit $\$ 36$ and indlcate the categories which best describe your home. Please indicate all that apply.

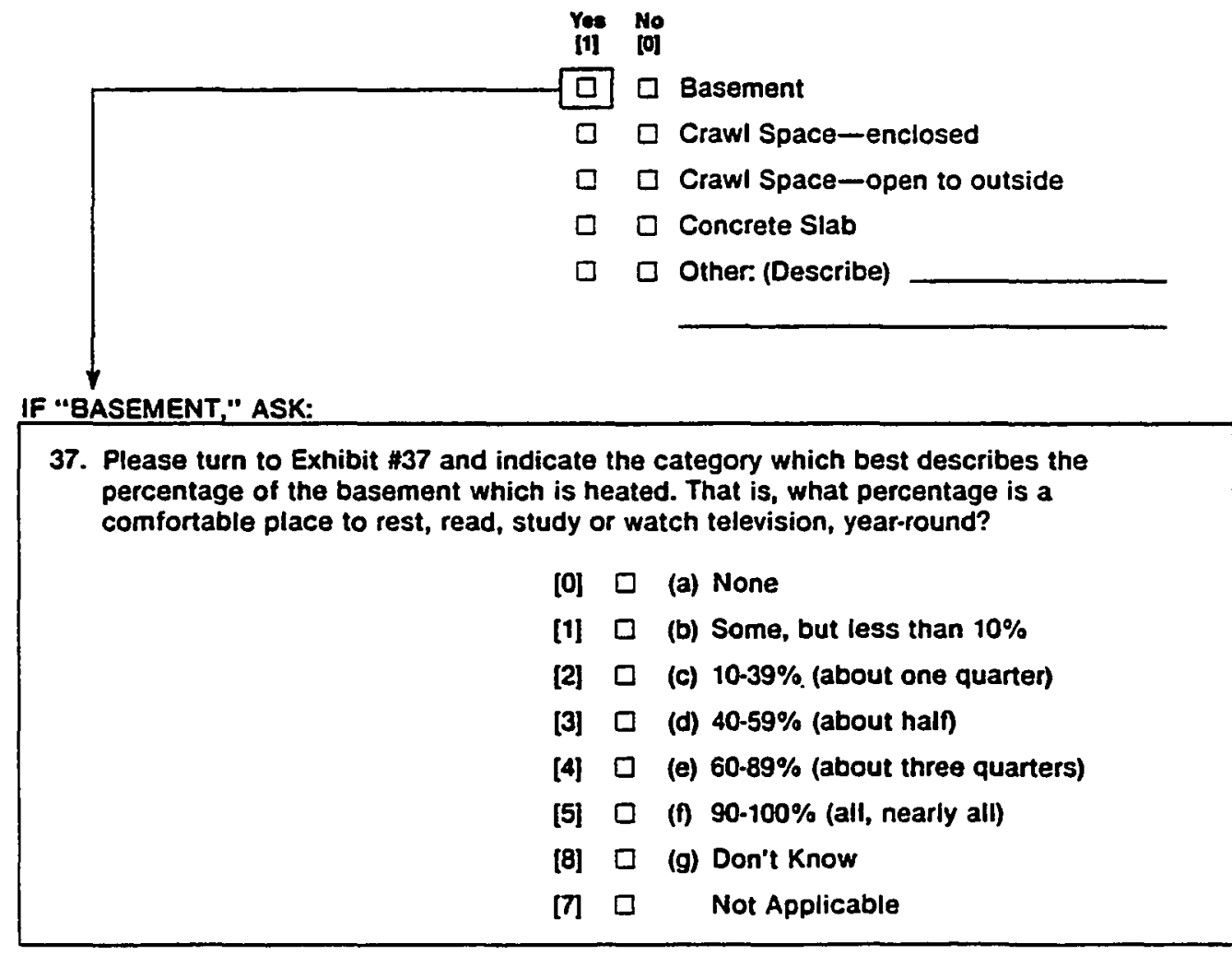




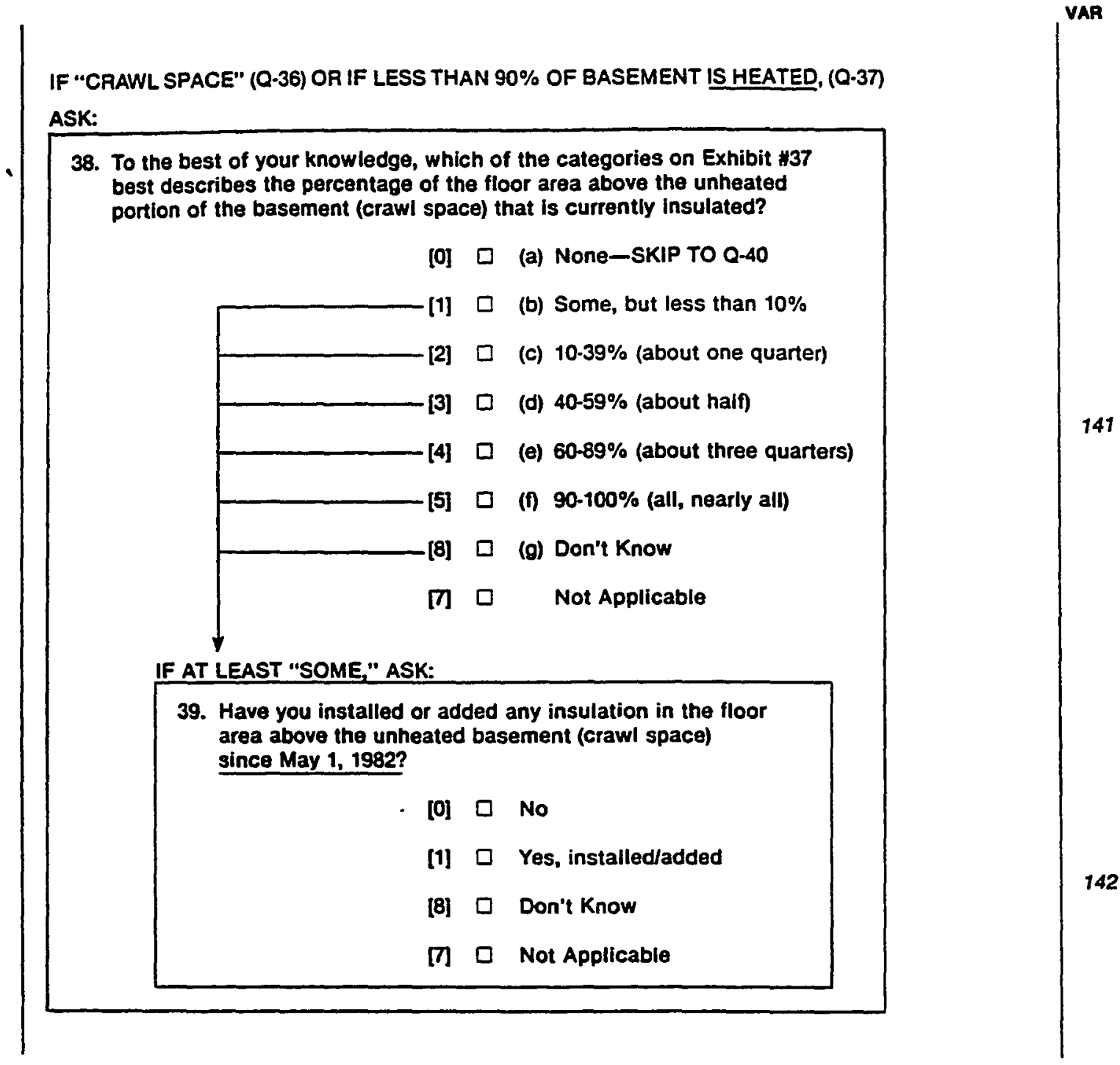


IF BETWEEN "SOME" AND "ALL" OF THE BASEMENT IS HEATED, (Q-37) ASK:

VAR

40. To the best of your knowledge, which of the categories on Exhibit \#37 best describes the percentage of the walls (of the heated portion) of the basement that currently contain insulation?

[0] $\square$ (a) None-SKIP TO Q-42

[1] $\square$ (b) Some, but less than $10 \%$

[2] $\square$ (c) $10-39 \%$ (about one quarter)

[3] $\square$ (d) $40-59 \%$ (about half)

[4] $\square \quad$ (e) $60-89 \%$ (about three quarters)

143

[5] $\square$ (f) $90-100 \%$ (all, nearly all)

[8] $\square$ (g) Don't Know

[7] $\square$ Not Applicable

IF AT LEAST "SOME" ASK:

41. Have you installed or added any insulation to the basement walls since May 1, 1982?
[0] $\square$ No
[1] $\square$ Yes, installed/added
[8] Don't Know
[7] $\square$ Not Applicable 
42. Excluding sliding glass doors, how many doors are there in your home that lead directly from a heated area to the outside or to an unheated porch or unheated garage?

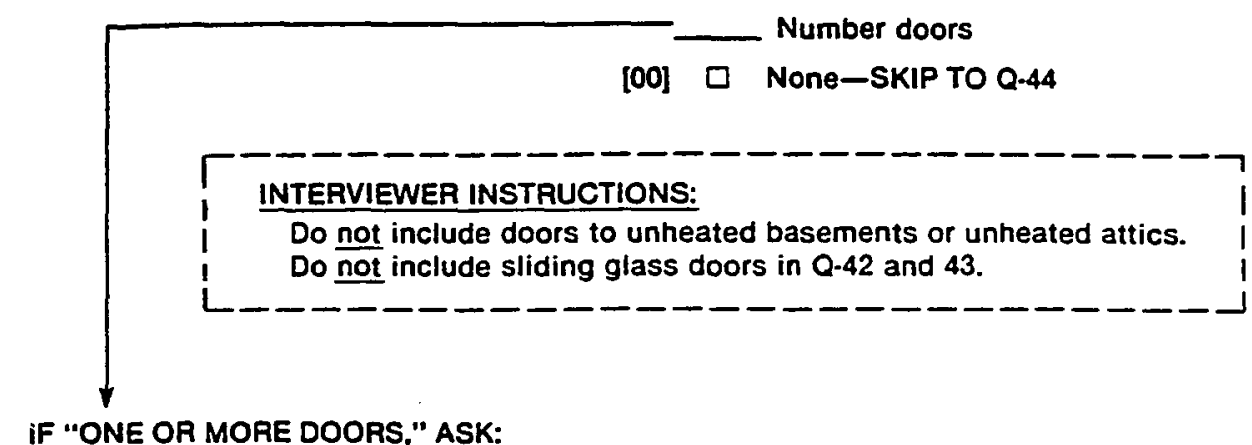

145

IF "ONE OR MORE DOORS" ASK:

43. How many of these doors have storm doors installed during the months when heating is required?

[00] $\square$ None

[97] $\square$ Not applicable

44. How many sliding glass doors are there in your home that lead directly from a heated area to the outside or to an unheated porch or unheated garage?

Number sliding glass doors

147

[00] $\square$ None-SKIP TO Q-46

IF "ONE OR MORE SLIDING GLASS DOORS" ASK:

45. How many of these sliding glass doors contain insulating glass or have storm doors installed during the months when heating is required?

Number insulated glass or storm doors

148

[00] $\square$ None

[97] $\square$ Not applicable 
46. Please reveiw Exhibit \#46 and indicate which of the categories on the list apply to the windows in your home during the months when heating is required. READ LIST

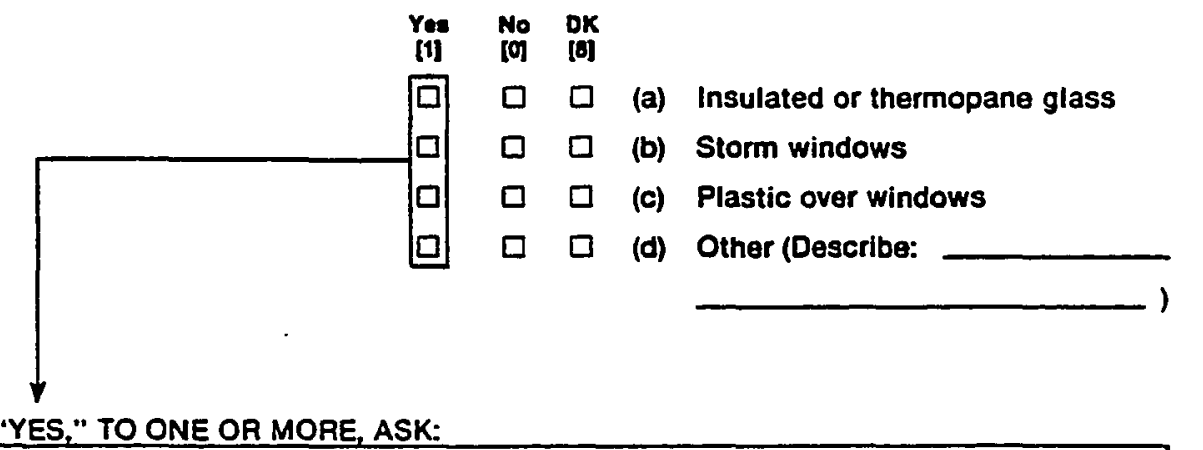

IF "YES," TO ONE OR MORE, ASK:

47. Now would you please look at Exhibit \#47 and indicate the category which best describes the percentage of windows which have... READ EACH ITEM

(a) Insulated/thermopane glass.

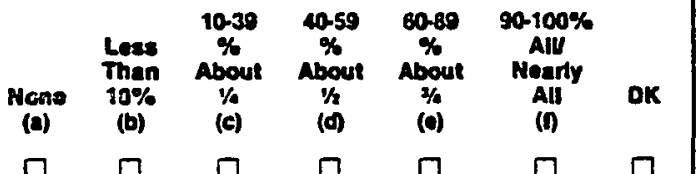

(b) Storm windows

(c) Plastic over windows...... $\quad \square \quad \square \quad \square \quad \square \quad \square \quad \square \quad \square \quad \square \quad \square \quad \square \quad 155$

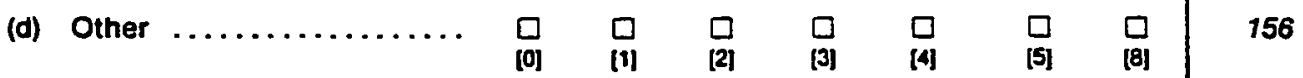


Now I would like to ask you a few questions about how you heat your home.

VAR

48. Would you please turn to Exhibit \#48 and indicate which one of the fuels listed is used most of the time to heat your home?

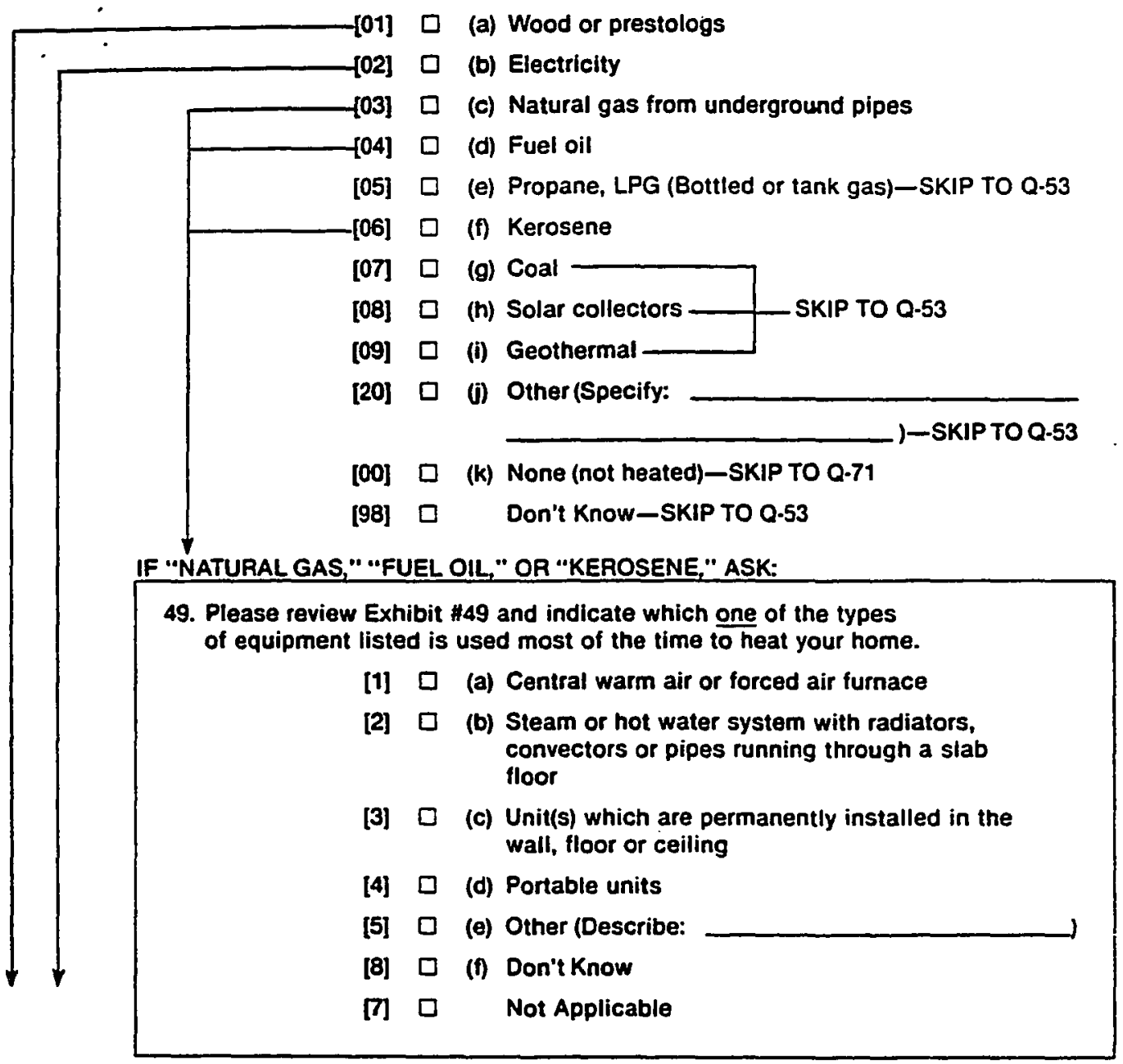




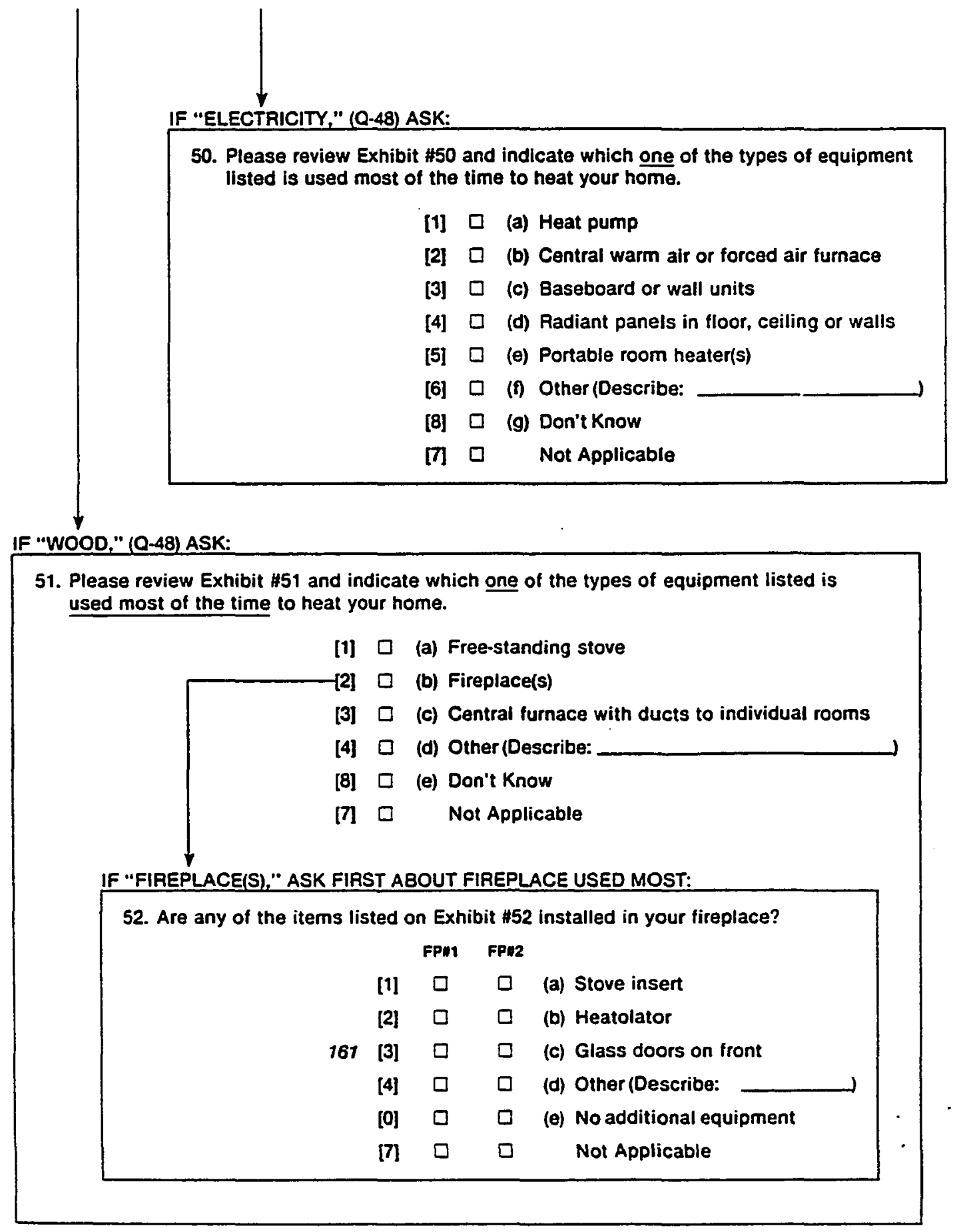


IF 2 OR MORE HOUSING UNITS IN BUILDING, ASK:

53. Is your home heated by a central system for your building (or group of buildings) or is the main heating equipment for your living quarters only?

[1] $\square$ Central system for building(s)

[2] $\square$ Main heat equipment for these living quarters only

[8] $\square$ Don't Know

[7] $\square$ Not Applicable

\section{ASK EVERYONE:}

54. Since September 1981 have you changed the fuel type you use most of the time to heat this house (apartment)?

[0] $\square$ No

164

[1] $\square$ Yes

[7] $\square$ No Fuel Used

[8] $\square$ Don't Know

IF "YES," ASK:

55. In what month and year did you make this change? [If month is DK, code "00."]

$\begin{array}{ll} & \text { Month } \\ \text { Year } \\ {[97] \quad \text { Don't Know-Month and Year }}\end{array}$

56. Please look at Exhibit \#56 and indicate which fuel was used most of the time to heat this home before September 1981?

$\begin{array}{lll}{[01]} & \square & \text { (a) Wood or prestologs } \\ {[02]} & \square & \text { (b) Electricity } \\ {[03]} & \square & \text { (c) Natural gas from underground pipes } \\ {[04]} & \square & \text { (d) Fuel oil } \\ {[05]} & \square & \text { (e) Propane, LPG (Bottled or tank gas) } \\ {[06]} & \square & \text { (f) Kerosene } \\ {[07]} & \text { (g) Coal } \\ {[08] \square} & \text { (h) Solar collectors } \\ {[09] \square} & \text { (i) Geothermal } \\ {[20] \square} & \text { (j) Other (Describe: } \\ {[00] \square} & \text { (k) None (not heated) } \\ {[98] \square} & \text { Don't Know } \\ {[97] \square} & \text { Not Applicable }\end{array}$


57. Was the heating equipment you currently use most of the time already installed in this home when you (member[s] of your household) first moved in?

$$
\begin{aligned}
& \text { [0] } \square \text { No } \\
& \text { [1] } \square \text { Yes } \\
& \text { [8] } \square \text { Don't Know }
\end{aligned}
$$

58. In what year was the heating equipment you now use most of the time installed in this home?

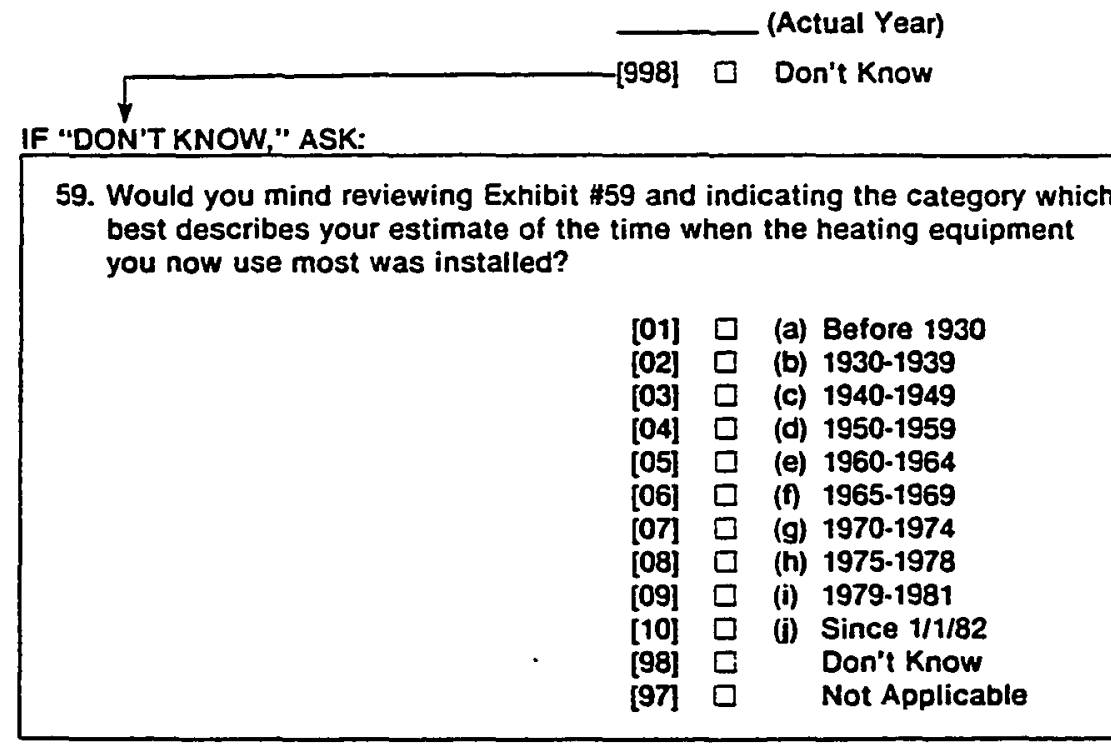


60. You have already mentioned the fuel which you use most of the time to heat your home. Do you use any other fuels to heat your home in addition to the fuel you use most of the time?

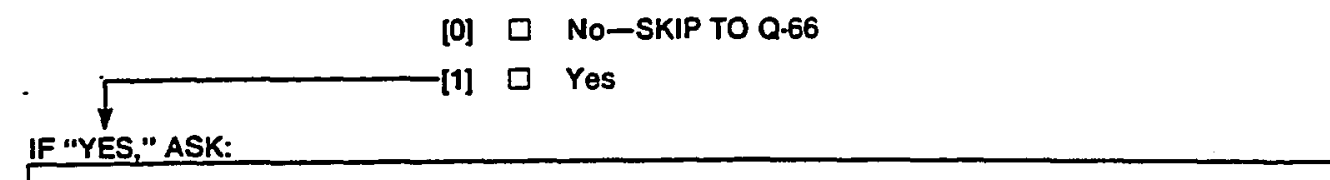$$
\text { [0] } \square \text { No-SKIP TO Q.66 }
$$$$
\text { IF "YES," ASK: }[1] \square \text { Yes }
$$

61. Please look at Exhibit \#61 and indicate which fuels you use for heating this home in addition to the fuel used most of the time.

Please indicate all of the fuels used.

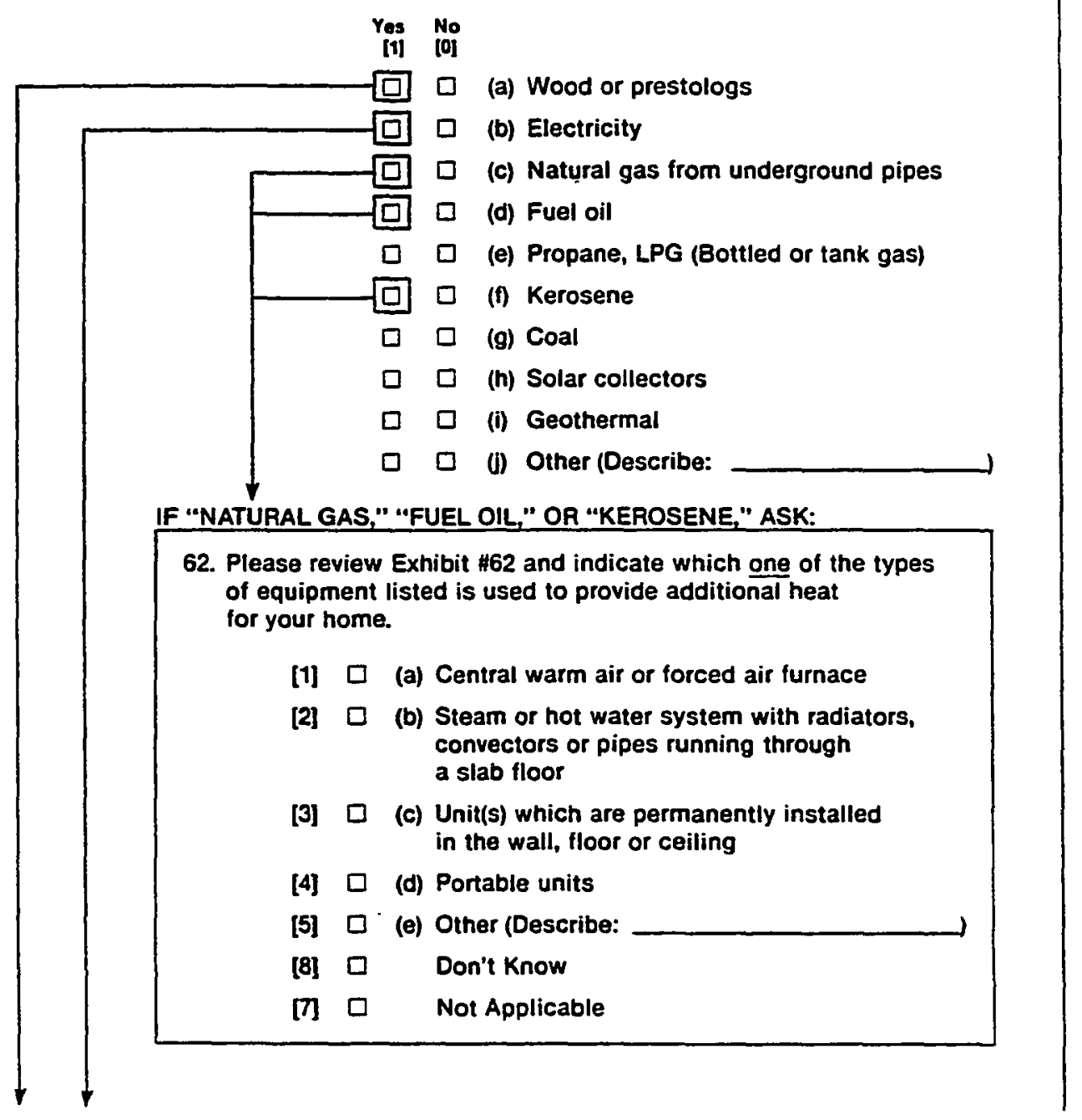




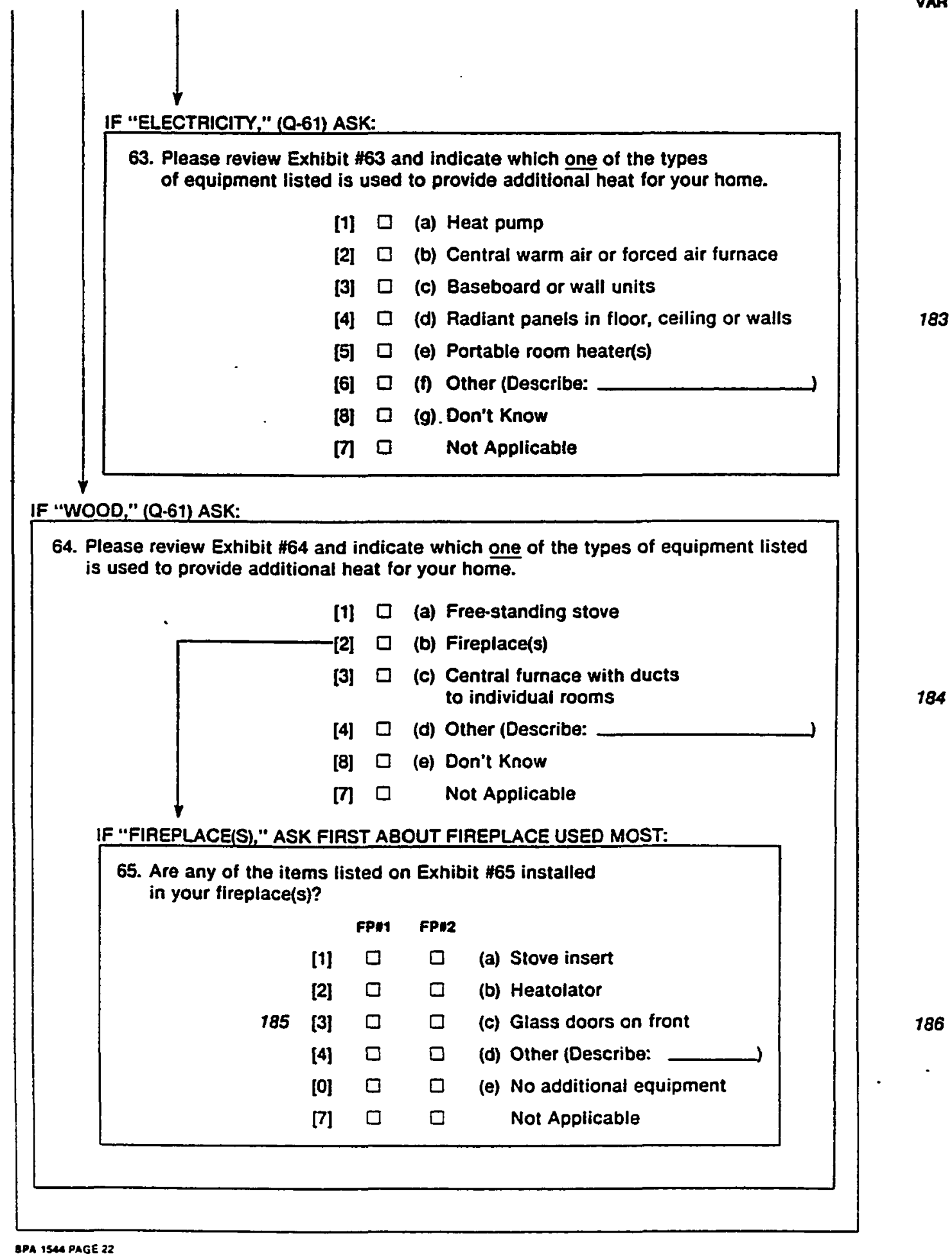


66. Have you burned any wood or prestologs in your home in the past 12 months? [Probe for type.]

[0] $\square$ No-SKIP TO Q-70

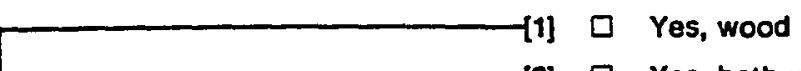

IF "WOOD," ASK:

68. Using the illustration on Exhibit 668 , would you please estimate how many cords of wood you burned in the past 12 months?

Number of cords

[9998] $\square$ Don't Know

[9997] $\square$ Not Applicable

INTERVIEWER INSTRUCTIONS:

Probe for respondent's best estimate of number of cords burned.

Record answer to nearest fraction of a cord $-.25,1.00,1.50,3.00$,

$6.00,12.00$.

69. Now please turn to Exhibit $\# 69$ and indicate the category that best describes your estimate of the percentage of this wood that you purchased.
[0] $\square$ (a) None
[1] $\square$ (b) Some, but less than $10 \%$
(2) $\square$ (c) $10-39 \%$ (about one quarter)
[3] $\square$ (d) $40-59 \%$ (about half)
[4] $\square$ (e) $60-89 \%$ (about three quarters)
[5] $\square$ (f) $90-100 \%$ (all, nearly all)
[8] $\square$ Don't Know
[7] $\square \quad$ Not Applicable 
70. Do you use a thermostat, radiator valve, on-off switch or some other device to control the temperature in your home most of the time during the months when heating is required?

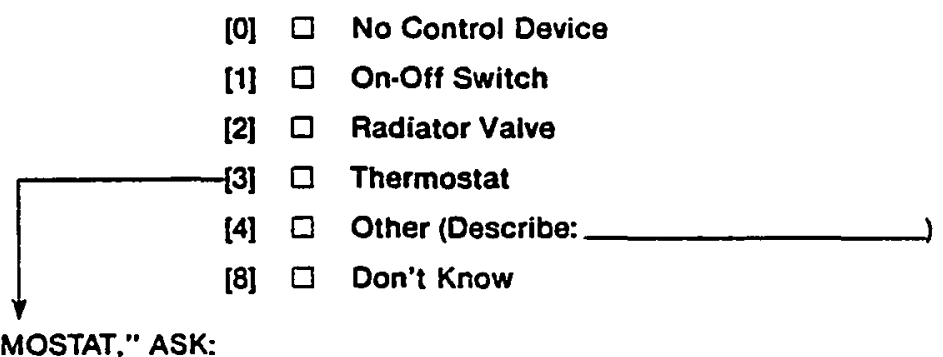

IF "THERMOSTAT," ASK:

71. Is this an automatic clock thermostat or does the thermostat have a timer?
[0] $\square$ No
[1] Yes, clock
[2] $\square$ Yes, timer
[8] $\square$ Don't Know
[7] $\square$ Not Applicable

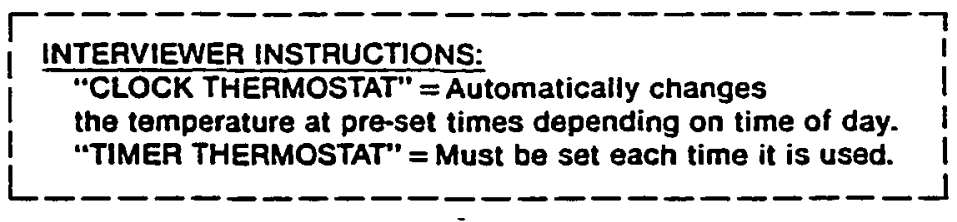


72. Now l'm going to ask you a few questions about the temperature you usually keep your home during the months when heating is required. Just your estimate or a range of temperatures will be fine. Please also te!l $m e$ if you turn the heat off.

(a) When someone is at home during the day or evening in the heating months? (SEE INSTRUCTION BELOW.)

$$
194 \begin{aligned}
& - \text { Degrees Fahrenheit } \\
& {[97] \text { to } \square \text { Heat turned off }} \\
& {[98] \square \text { Don't Know }}
\end{aligned}
$$

(b) When no one is at home during the day or evening in the heating months? (SEE INSTRUCTION BELOW.)

$197 \begin{aligned} & \text { Degrees Fahrenheit } \\ & {[97] \quad \square \quad \text { Heat turned off }} \\ & {[98] \square \text { Don't Know }}\end{aligned}$

(C) During sleeping hours in the heating months? (SEE INSTRUCTION BELOW.)

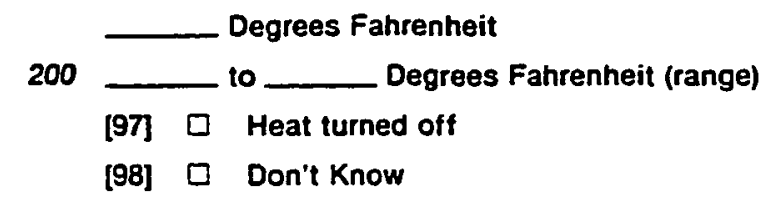


73. During the months when heating is required, is someone usually home and the house heated at

VAR least half of the time on weekdays between the hours of 9:00 a.m. and 5:00 p.m.?

$$
\begin{aligned}
& \text { [0] } \square \text { No } \\
& \text { [1] } \square \text { Yes } \\
& \text { [9] } \square \text { Refused }
\end{aligned}
$$

74. In order to help us better understand how you use energy in your home, would you mind telling us whether, since September 1981, there have been periods of at least one week when you did not use any of your appliances, heating or cooling equipment, or any other large equipment, because you were away on vacation, on business, were hospitalized, or for some other reason?

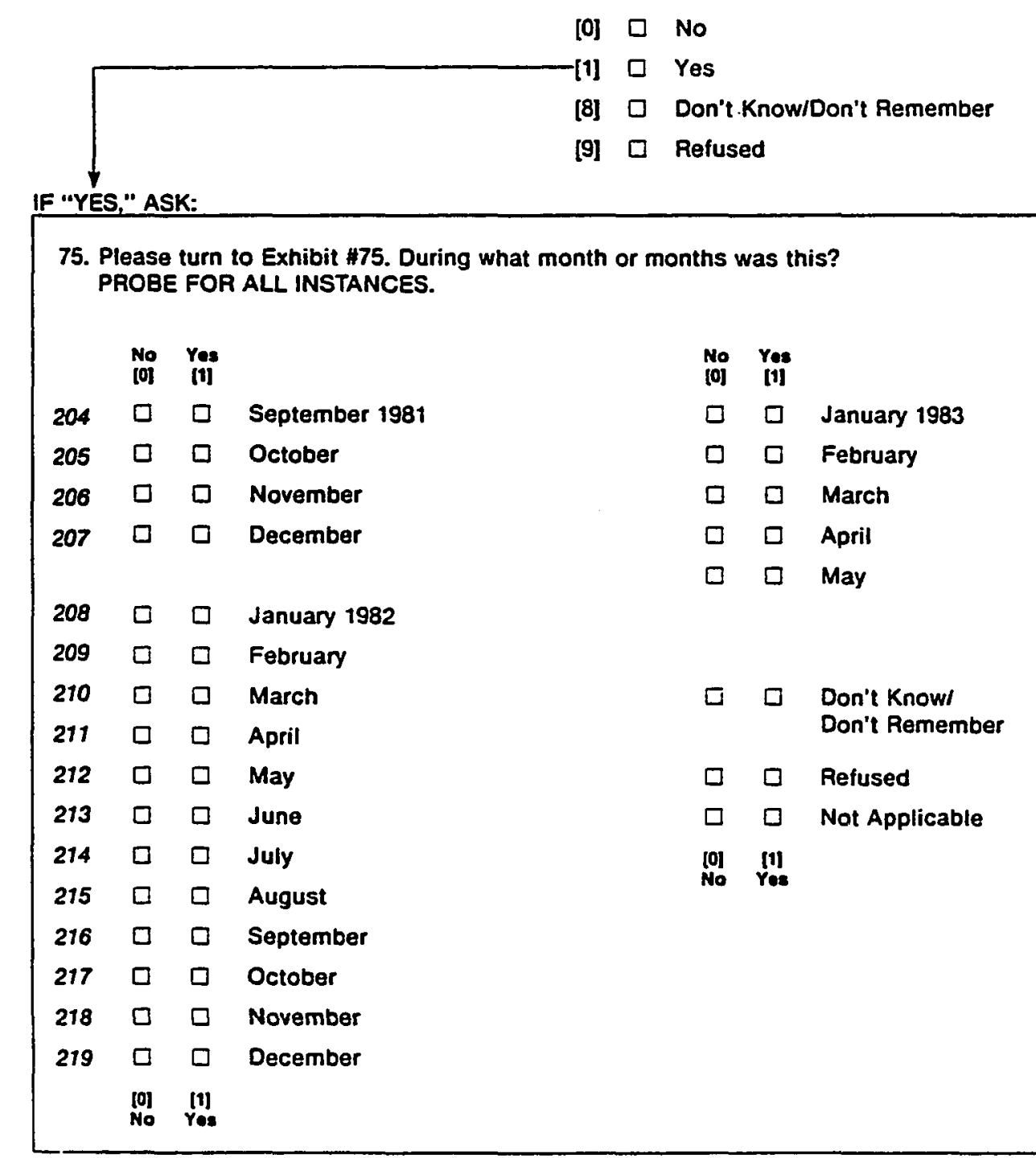


76. Now I would like to ask you some questions about how your water is heated.

VAR

Please turn to Exhibit \#76 and indicate which fuel is used most for heating water in your home.
[01] $\square$ (a) Wood
[02] $\square$ (b) Electricity
[03] $\square$ (c) Natural gas from undergound pipes
[04] $\square$ (d) Fuel oil
[05] $\square$ (e) Propane, LPG (Bottled or tank gas)
[06] $\square$ (f) Kerosene
[08] $\square \quad$ (g) Solar collectors
[10] $\square$ (h) Heat pump
[20] $\square$ (i) Other (Describe:
[00] $\square$ (j) None-no hot water-SKIP TO Q-91
[98] $\square$ Don't Know

IF 2 OR MORE HOUSING UNITS IN BUILDING, ASK:

77. Is your hot water supplied by a central system for your building

(or group of buildings) or is the water heater for your living quarters only?
[1] $\square$ Central system for building(s)
[2] $\square$ For these living quarters only
[8] Don't Know
[7] Not Applicable

78. Was the water heating equipment you currently use most already installed in your home when you (member[s] of your household) first moved in?
[0] $\square$ No
[1] $\square$ Yes
[8] $\square$ Don't know

$\left\{\begin{array}{l}\text { INTERVIEWER INSTRUCTIONS: } \\ \text { Installation of a new hot water tank is considered } \\ \text { "new water heating equipment." } \\ \text { Installation of a now burner only is not considered } \\ \text { "new water heating equipment." }\end{array}\right.$


79. In what year was the water heating equipment you currently use most installed in your home? (Actual Year)

\begin{tabular}{|c|c|c|c|c|c|}
\hline \multicolumn{6}{|c|}{$\begin{array}{l}\text { 80. Would you mind looking at Exhibit } \# 80 \text { and indicating which of the categories } \\
\text { best describes the time when the water heating equipment was installed? }\end{array}$} \\
\hline [01] & $\square$ & (a) Before 1960 & [06] & $\square \quad(n)$ & $1979-1981$ \\
\hline [02] & $\square$ & (b) $1960-1964$ & {$[07]$} & $\square \quad(g)$ & Since $1 / 1 / 82$ \\
\hline [03] & $\square$ & (c) $1965-1969$ & [98] & $\square$ & Don't Know \\
\hline [04] & $\square$ & (d) $1970-1974$ & {$[97]$} & $\square$ & Not Applicable \\
\hline [05] & $\square$ & (e) $1975-1978$ & & & \\
\hline
\end{tabular}

81. Is the water heater located in an area that is normally heated-or an area normally not heated?

$$
\begin{aligned}
& \text { [1] } \square \text { Heated area } \\
& \text { [2] } \square \text { Not heated area } \\
& \text { [8] } \square \text { Don't Know }
\end{aligned}
$$

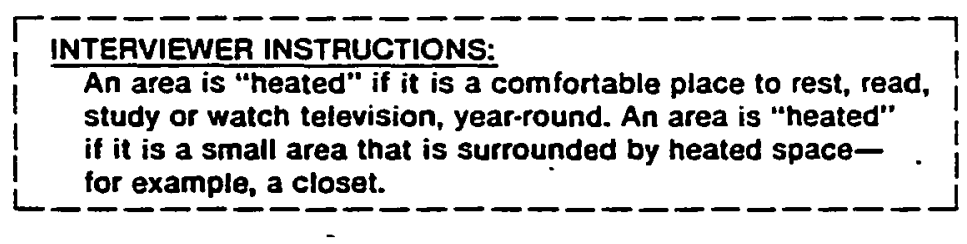

82. Does that water heater have an extra layer of insulation wrapped around the outside of the tankin the form of a mat or jacket or blanket?

IF "YES," ASK:

83. Was the wrap (will the wrap be) provided by your fuel supplier (utility), did you purchase it yourself, or was it obtained from some other source?
[1] $\square$ Supplier
[2] $\square$ Self
[3] $\square$ Other
[8] Don't Know 
84. In addition to your main fuel, do you use any other fuel for heating water?

VAR

[0] $\square$ No-SKIP TO Q-86

[1] $\square$ Yes

- IF "YES," ASK:

85. Please indicate what this additional fuel is, using Exhibit $\# 85$.

[01] $\square \quad$ (a) Wood

[02] $\square$ (b) Electricity

[03] $\square$ (c) Natural gas from underground pipes

[04] $\square$ (d) Fuel oil

[05] $\square$ (e) Propane, LPG (Bottled or tank gas)

[06] $\square$ (f) Kerosene

237

[08] $\square \quad$ (g) Solar collectors

[10] $\square$ (h) Heat pump

[20] $\square$ (i) Other (Describe:

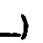

[98] $\square$ Don't Know

[97] $\square \quad$ Not Applicable 
86. How many shower facilities, if any, are there in your home? Include all showers, even those not used.

Number of showers

[0] $\square$ None-SKIP TO Q-88

IF "ONE OR MORE SHOWERS," ASK:

87. How many of these showers, if any, have a flow restrictor on the outlet, either low-flow showerheads or low-flow inserts?

Number, low-flow showerheads
Number, low-flow inserts
INT $\square$ Don't Know
Probe for usage.

88. How many of the sink faucets in your home have low-flow devices installed?

$\overline{[00] \square}$ None

89. Have you ever permanently removed any low-flow shower or faucet devices in your home?

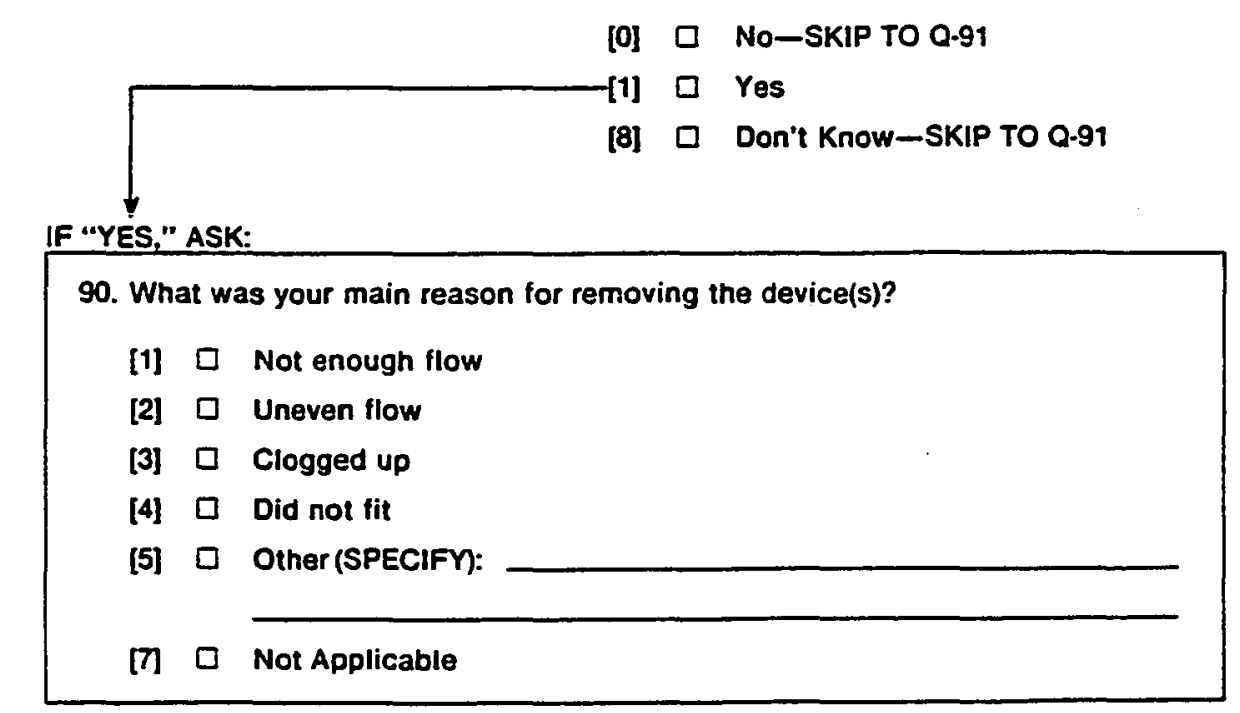



IF "YES," ASK:

92. From the list on Exhibit \$92, please indicate what type of air conditioning system you have in your home.

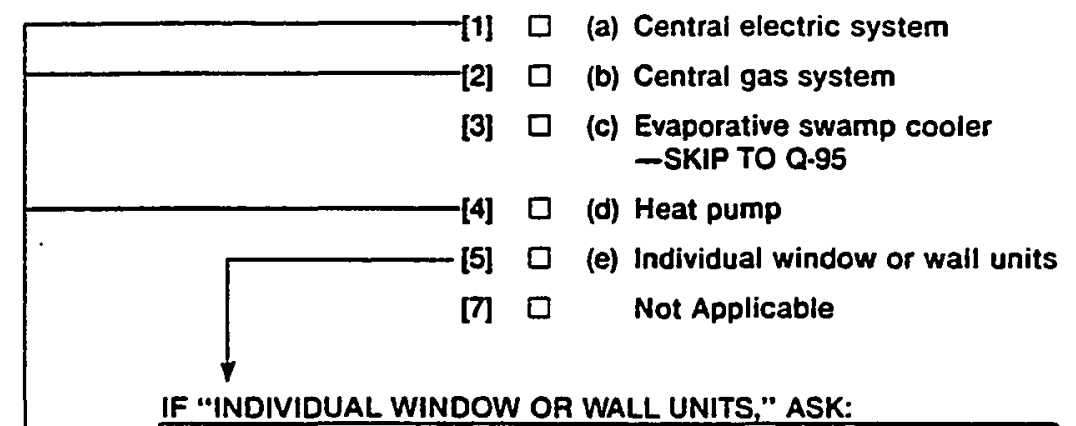

93. How many window or wall units do you have in your home?

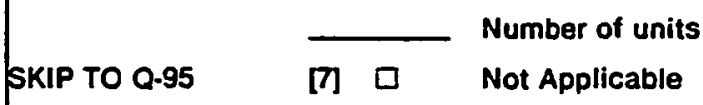


VAR

INTERVIEWER INSTRUCTIONS:

For each of the following questions, treat "Central System" as "AC\#1." Treat "Window or Wall Unit" which is used most as "AC\#1" and, if present, the second most used as "AC"\#2."

(1)

95. Which of the statements on Exhibit \#95 best describes the way you used your air conditioner(s) during the months when cooling may be required?

Did not use at all ......................................

[0]

249

(b) Turned on only a few days or nights when really needed ........... $\square \square$ [1]

(c) Turned on a moderate amount of time during the cooling season ...... $\square \quad \square \quad$ [2]

(d) Turned on just about all of the cooling season ................ $\square \square \square$

(e) Other (Describe: ___ _,$\square \quad \square[4]$

Not Applicable .............................. $\square \square[$ [

96. Was this air conditioning equipment already installed in your home when you (member[s] of your household) first moved in?

$$
\begin{array}{llll}
\text { No } \ldots \ldots \ldots \ldots . .59 & \square & \square & {[0]} \\
\text { Yes } \ldots \ldots \ldots \ldots . & \square & \square & {[1]} \\
\text { Not Applicable.. } & \square & \square & {[7]}
\end{array}
$$

97. Please turn to Exhibit $\$ 97$ and indicate the category which best describes how many years old this air conditioning equipment is.

(a) Less than 1 year .. 25?? $\square \quad \square \quad[1]$

(b) 1 to 2 years $\ldots \ldots \ldots \square \square \square$ [2]

(c) 3 to 5 years $\ldots \ldots \ldots \square \square \square$ [3]

(d) 6 to 10 years $\ldots \ldots \ldots \square \square \square \quad[4]$

(e) More than 10 years ... $\square \quad \square$ [5]

Don't know ......... $\square \quad \square \quad[8]$

Not Applicable ...... $\square \square$ [7] 
Now I would like to ask you some questions about the appliances you have here in your home.

The first few questions are about your televisions.

98. How many televisions do you have that you use here in your home, at least occasionally?

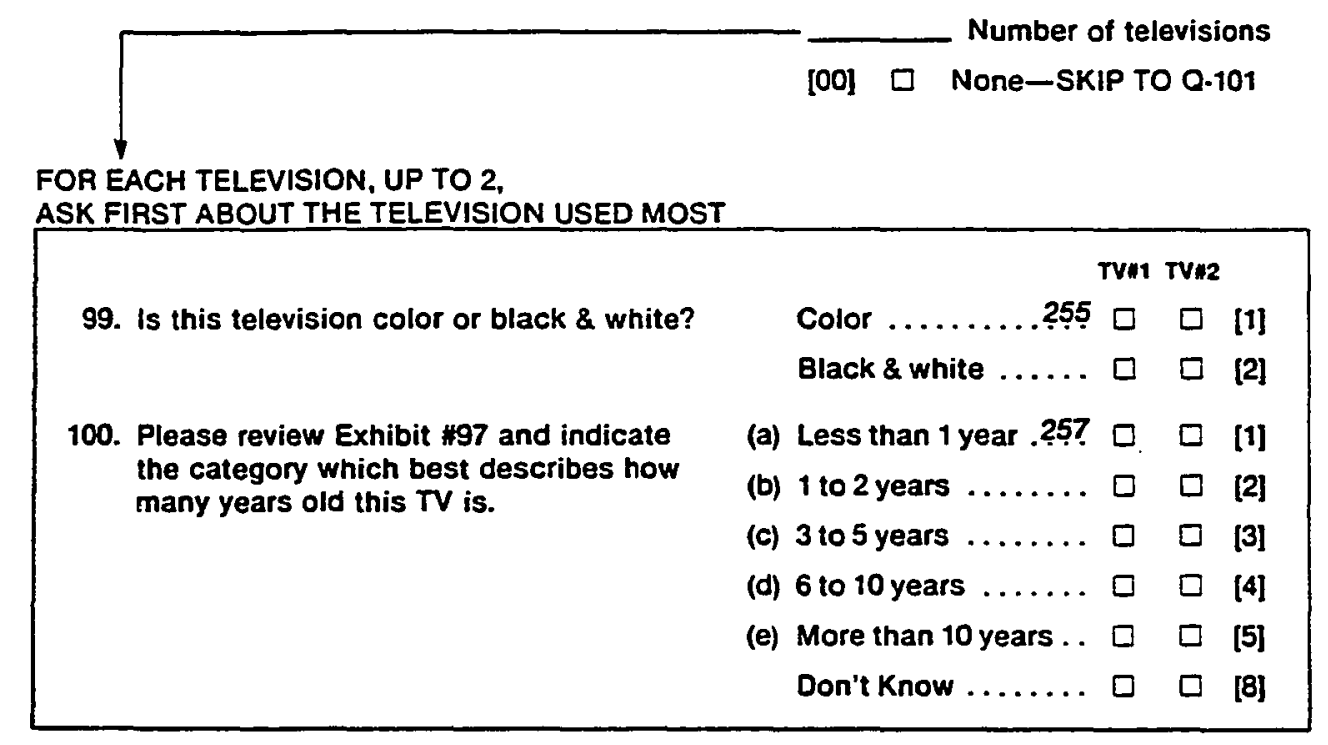


The next few questions are about the appliances you use for cooking.

101. What fuel do your stove top burners use?
[01] $\square$ Wood
[02] $\square$ Electricity
[03] $\square$ Natural gas from underground pipes
[04] $\square$ Propane, LPG (Bottled or tank gas)
[06] $\square$ Kerosene
[20] $\square$ Other (Describe):
[00] $\square$ None-no burners-SKIP TO Q.104

102. Would you please look at Exhibit \#102 and indicate the category which best describes when the stove top burners were installed in your home.
[1] (a) Already installed when I moved in
[2] $\square$ (b) Brought with me when I moved in
[3] $\square$ (c) Purchased by me when I moved in or since I moved in
[4] $\square$ (d) Purchased by someone else when I moved in or since I moved in
[7] $\square \quad$ Not Applicable

103. Now would you please furn to Exhibit \#103 and indicate which of the categories best describes your estimate of how many years old your stove top burners are?
[1] $\square$ (a) Less than 1 year
[2] $\square \quad$ (b) 1 to 2 years
[3] $\square$ (c) 3 to 5 years
[4] $\square \quad$ (d) 6 to 10 years
[5] $\square \quad$ (e) More than 10 years
[8] $\square$ Don't Know
[7] $\square \quad$ Not Applicable 
104. How many ovens, including microwave or convection ovens, do you have that you use for

VAR cooking, at least occasionally? Please consider each oven separately, regardless of whether they are combined in a single unit. For example, a double-oven unit counts as two ovens. Please do not include toaster ovens.

Number of ovens

[0] $\square$ None-SKIP TO Q-111

$\checkmark$

FOR EACH OVEN, UP TO 2.

ASK FIRST ABOUT THE OVEN USED MOST

Oven "1 Oven "2

105. Is this oven electric, gas, or does it use some other fuel?

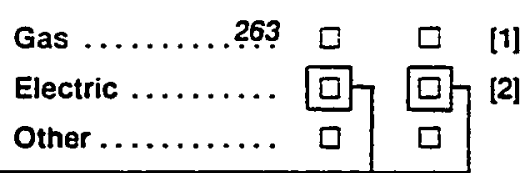

$\checkmark$

IF "ELECTAIC," ASK:

106. Is it a microwave oven?

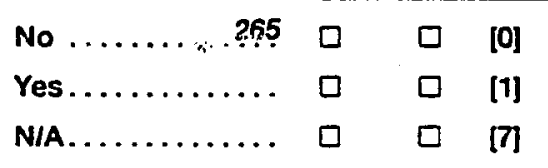

266

107. Is this oven separate or is it combined with your stove top burners in a single

Separate ..... 26? $\square \quad \square \quad$ [1]

Combined .........

[2] unit?

108. Please look at Exhibit \#108 and indicate the category which best describes when the oven was installed in your home.

(a) Already installed when I moved in

$n \ldots \ldots \ldots \ldots+\ldots \ldots$

(b) Brought with me when I moved in

$\square \quad \square \quad[1]$

(c) Purchased by me when I moved in or since I moved in .....

$\square \quad$ [2]

(d) Purchased by someone else when I moved in or since I

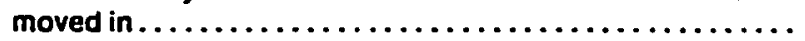

109. Now would you please review Exhibit \#109 and indicate the category which best describes your estimate of how many years old the oven is?

(a) Less than 1 year . 27?!

110. Is it a self-cleaning oven?
(b) 1 to 2 years $\ldots \ldots \ldots$.
$\square \quad$ [2]
(c) 3 to 5 years ....... $\square \quad \square \quad$ [3]
(d) 6 to 10 years ...... $\square \quad \square \quad$ [4]
(e) More than 10 years .. $\square \quad \square \quad$ [5]
Don't know ........ $\square \quad \square \quad[8]$
No.........?? $\square$ ? $\square$ [1]

$\square \quad[1]$ 
Now I would like to ask you a few questions about your refrigerators and freezers.

111. How many refrigerators do you have that are presently in use?

VAR Number presently in use

[0] $\square$ None-SKIP TO Q-115

FOR EACH REFRIGERATOR, UP TO 2

ASK FIRST ABOUT THE REFRIGERATOR USED MOST

112. How large is this refrigerator?

Rotito $* 1$ Rofrig $* 2$

- Large (17 cubic feet or more) 276

- Medium (10 to 16 cubic feet) ....

- Small (less than 10 cubic feet) ..

113. Which of the following best describes the location of the freezer section?

$\begin{array}{llll}\text { - Side-by-side ......??ళ } & \square & \square & \text { [1] } \\ \text { - Top ............... } & \square & \square & \text { [2] } \\ \text { - Bottom............ } & \square & \square & {[3]} \\ \text { - Inside.............. } & \square & \square & {[4]} \\ \text { - No freezer section ... } & \square & \square & {[5]}\end{array}$

114. Is this refrigerator "frost-free" or must it be manually defrosted?
- Frost-free....... 2890
- Manual defrost
- No freezer section ...

115. How many freezers which are separate from your refrigerator do you have that are presently in use?

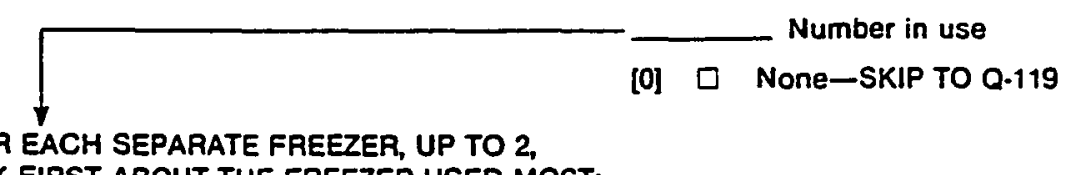

Freezer 11 Freezer 12

116. How large is this freezer?

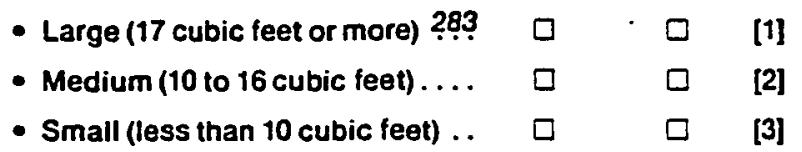

117. Is this an upright freezer or a horizontal (chest-type) freezer?

- Upright........ 28̣5 $\quad \square \quad \square \quad[1]$

- Horizontal (chest) .... $\quad \square \quad \square \quad$ [2]

118. Is the freezer "frost-free" or is it "manual defrost"?

- Frost-free.......28? $\square \quad \square \quad[1]$ 
Next, I would like to know about some of the major home appliances which you use here in your home. ,

119. Would you please review the list of appliances on Exhibit \#119. For each of the appliances listed, please indicate whether you use the appliance on your own home electric or gas meter lines.

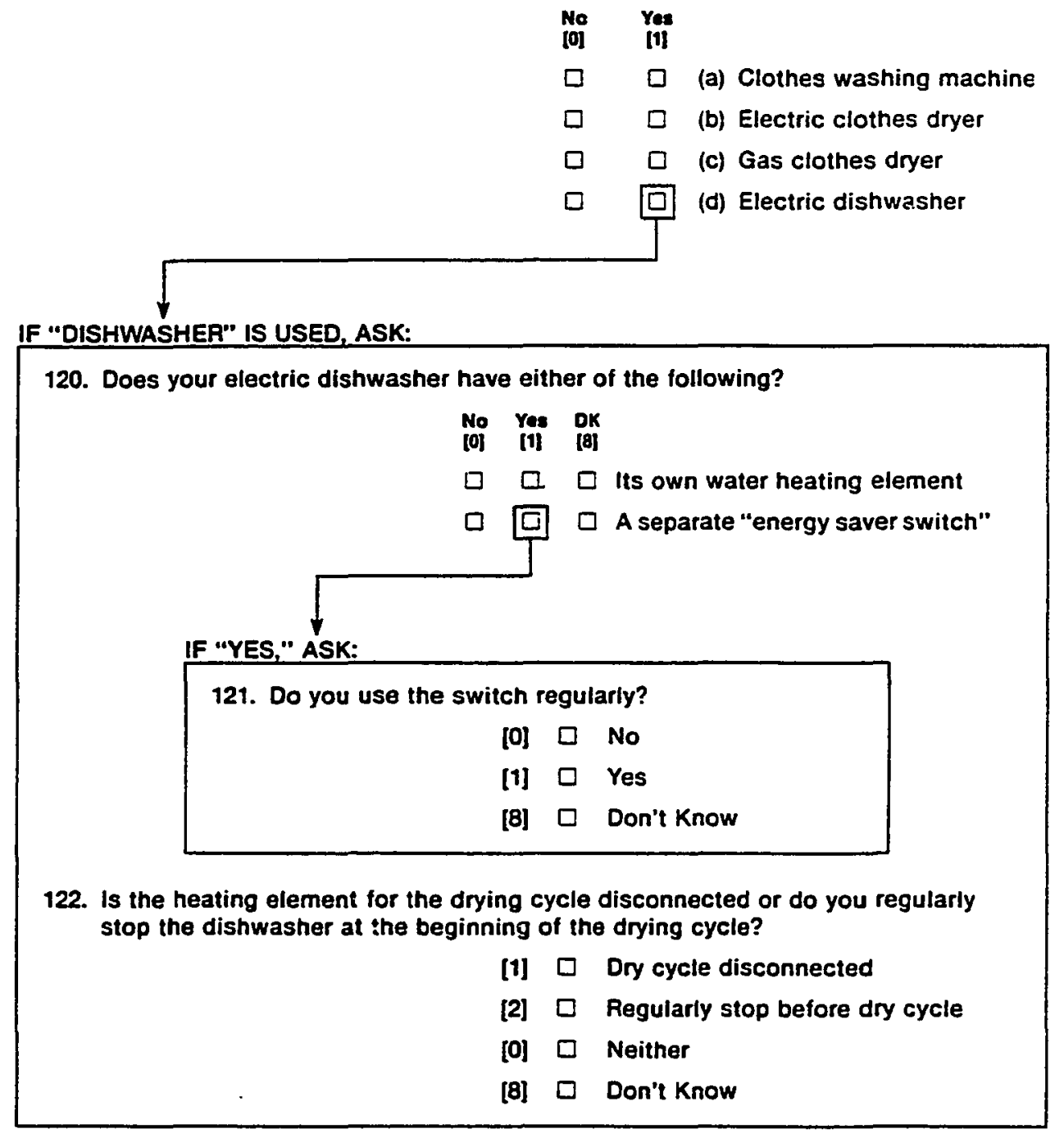

F "DISHWASHER" IS USED, ASK: 


\section{IF MOBILE HOME OR SINGLE FAMILY DWELLING, ASK:}

123. Do you have your own swimming pool, hot tub or Jacuzzi? MARK ALL THAT APPLY.

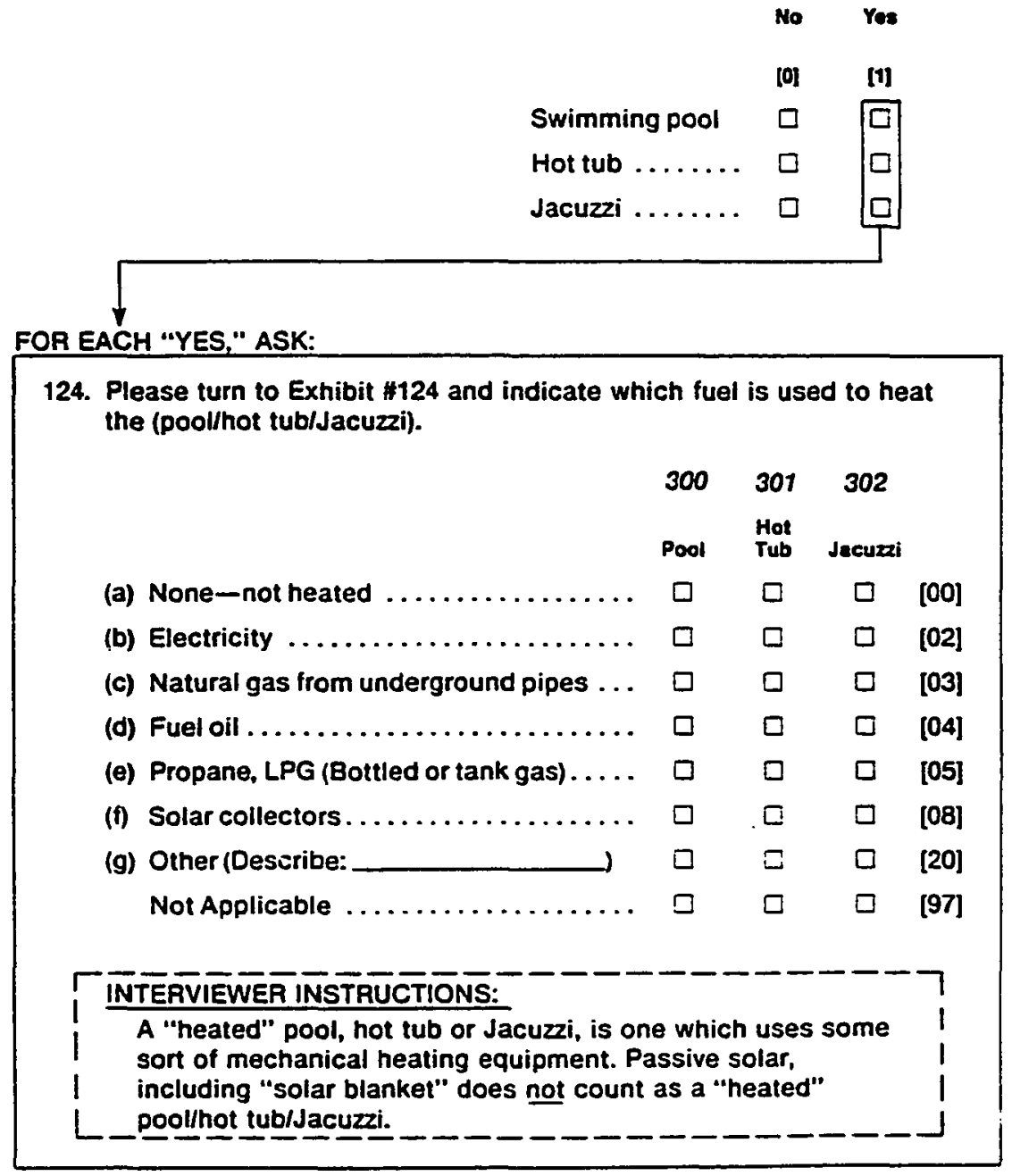


125. How many waterbed heaters do you have which are presently in use?

126. Would you please review the list of equipment on Exhibit \#126 and indicate whether you regularly use any of these types of equipment on your household electric or gas meter lines.

$x \cdot \cos$

\begin{tabular}{|c|c|c|}
\hline $\begin{array}{l}\text { No } \\
\text { [01 }\end{array}$ & $\begin{array}{l}\text { Yes } \\
\text { [1] }\end{array}$ & \\
\hline$\square$ & $\square(\mathrm{a})$ & Special large health or medical equipment \\
\hline$\square$ & $\square$ (b) & Photography laboratory equipment \\
\hline ᄃ & $\square(c)$ & Woodworking equipment (including saw, planers, etc.) \\
\hline$\square$ & $\square$ (d) & Electric ceramic kiln \\
\hline$\sqsubset$ & $\square(e)$ & Gas ceramic kiln \\
\hline ᄃ & $\square(f)$ & Home computer or video game \\
\hline$\square$ & $\square(g)$ & Electric office equipment \\
\hline$\square$ & $\square$ (h) & Metalworking equipment (including welding) \\
\hline 5 & $\square(\mathbf{i})$ & Lighting or heating for greenhouse \\
\hline$\square$ & $\square(\mathbf{j})$ & Water pumps for irrigation \\
\hline & $\square(\mathbf{k})$ & Electric well water pump for househol \\
\hline
\end{tabular}

304

305

306

307

308

309

310

311

312

313

314

127. Do any of your household electric or gas bills inctude charges for fuel used for purposes other than your own living quarters, such as farm buildings, or machinery, the house or apartment of another household, a business or office, or any other large uses?

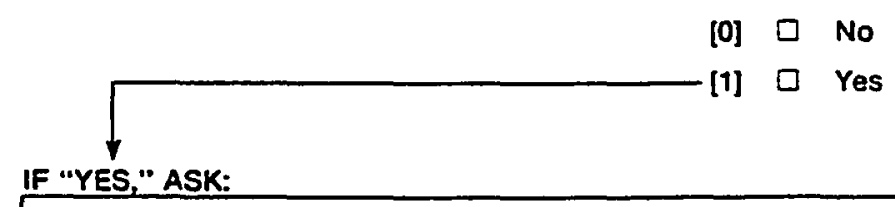

128. Which of your fuel bills include charges for fuel used for purposes other than your own living quarters?

\begin{tabular}{|c|c|c|}
\hline $\begin{array}{l}\text { No } \\
{[0]}\end{array}$ & $\begin{array}{l}\text { Yos } \\
\text { [1] }\end{array}$ & \\
\hline$\square$ & $\square$ (a) & Electricity \\
\hline$\square$ & $\square$ (b) & Natural gas from underground pipes \\
\hline
\end{tabular}


In order to help us better understand your answers to the questions about your home and how you use energy, and to help the Bonneville Power Administration to better design programs to fit the needs of different people, I have a few questions about yourself and the other people who live here.

129. First, how many persons, including yourself, normally reside herethat is, those who live here for more than half of the year?

Number of residents

130. Now, would you please tell me who the people are who live here-just in relation to yourself. I would also like to know the ages of each of the people.

If you prefer, you may use the list of age categories on Exhibit \#130.

\begin{tabular}{|c|c|c|c|c|c|}
\hline \multirow{2}{*}{$\begin{array}{c}\text { Porson } \\
\text { Number }\end{array}$} & \multirow{2}{*}{$\begin{array}{l}\text { Relietionshlp } \\
\text { to Respondent }\end{array}$} & \multirow{2}{*}{ Code } & Gendor & \multicolumn{2}{|c|}{ Age } \\
\hline & & & [0] [1] & Actual & Catogory \\
\hline 1 & PAIMARY RESPONDENT & $\begin{array}{ll}319 & \\
& 1\end{array}$ & $\begin{array}{cc}320 & \\
\square & \square\end{array}$ & 321 & 322 \\
\hline 2 & & 323 & $\begin{array}{r}324 \\
\square \quad \square\end{array}$ & 325 & 326 \\
\hline 3 & & 327 & $\begin{array}{cc}328 & \\
\square & \square\end{array}$ & 329 & 330 \\
\hline 4 & & 331 & $\begin{array}{c}332 \\
\square \quad \square\end{array}$ & 333 & 334 \\
\hline 5 & & 335 & $\begin{array}{cc}336 & \\
\square \quad \square\end{array}$ & 337 & 338 \\
\hline 6 & & 339 & $\begin{array}{l}340 \\
\square \quad \square\end{array}$ & 341 & 342 \\
\hline 7 & & 343 & $\begin{array}{c}344 \\
\square \quad \square\end{array}$ & 345 & 346 \\
\hline 8 & & 347 & $\begin{array}{c}348 \\
\square \quad \square\end{array}$ & 349 & 350 \\
\hline 9 & & 351 & $\begin{array}{cc}352 & \\
\square \quad \square\end{array}$ & 353 & 354 \\
\hline 10 & & 355 & $\begin{array}{c}356 \\
\square \quad \square\end{array}$ & 357 & 358 \\
\hline
\end{tabular}

BPA ISH PAGE \&O

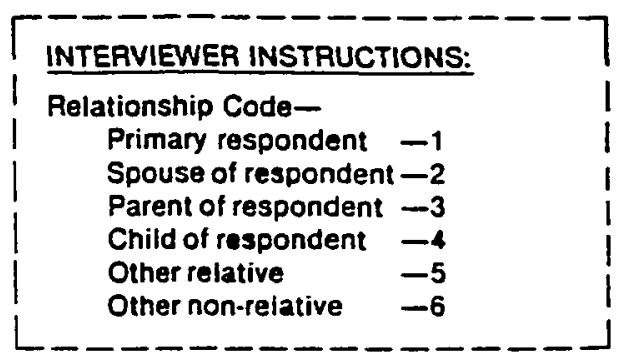


131. RECORD: Did HH\#2 actively participate in the interview?
[0] $\square$ No, not present
[1] $\square$ No, present but did not participate
[2] $\square$ Yes, part time
[3] $\square$ Yes, full time

132. Please review Exhibit \#132 and indicate which category of race and ethnic origin best describes (HOUSEHOLDER \#1). What about (HOUSEHOLDER \#2)?

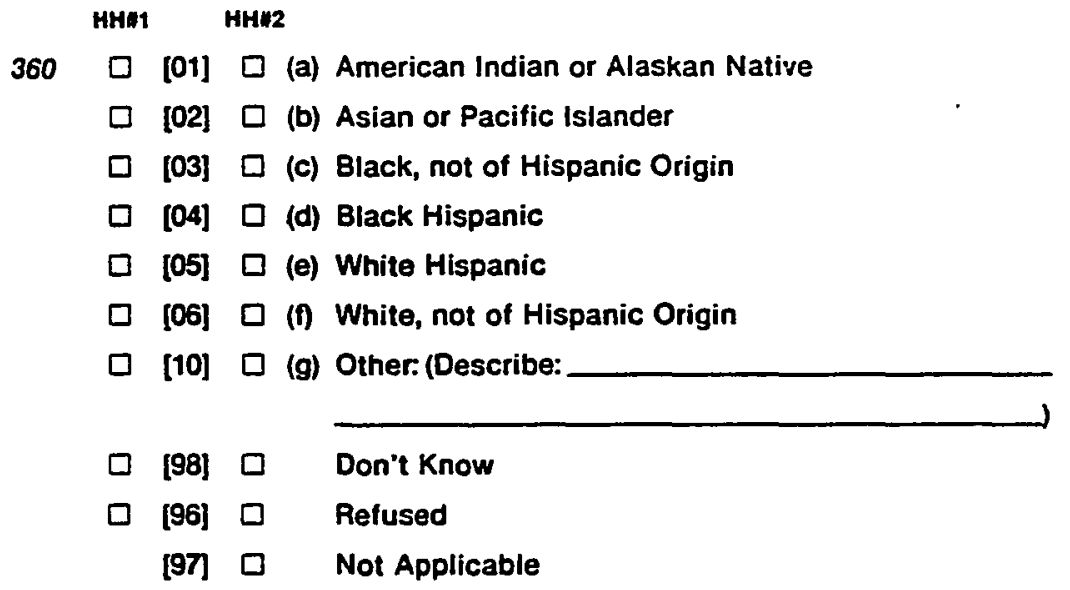

133. Please turn io Exhibit \#133 and indicate the category which best describes how much formal education (HOUSEHOLDER \#1) has received? Please just tell me the letter corresponding to the category which includes the last grade (or year) of school completed by (HOUSEHOLDER \#1). What about (HOUSEHOLDER "2)?

HHแ1 НHะ2

[01] $\square$ (a) Never attended school

$\square[02] \square$ (b) Some elementary school

$\square$ [03] $\square$ (c) Completed elementary school

$\square$ [04] $\square$ (d) Some high school

$\square$ [05] $\square$ (e) Completed high school

$\square$ [06] $\square$ (f) Completed trade/vocational school

ㅁ [07] $\square$ (g) Some college

$\square$ [08] $\square$ (h) Completed college

$\square \quad[09] \square$ (i) Some post-graduate

$\square$ [10] $\square$ (j) Completed post-graduate

[ [98] $\square$ Don't Know

$\square$ [99] $\square \quad$ Refused

[97] $\square \quad$ Not Applicable 
134. Now please turn to Exhibit \#134 and indicate the letter of the category which best describes the total combined income before taxes for your household for 1982.

(a) $\square$ [01]

(b) $\square \quad$ [02]

EXHIBIT \#134

(c) $\square$ [03]

(d) $\square$ [04]

(e) $\square$ [05]

(f) $\square$ [06]

364

(a) UNDER $\$ 5,000$

(g) $\square \quad$ [07]

(b) $\$ 5,000 \cdot \$ 7,999$

(h) $\square \quad$ [08]

(i) $\square \quad$ [09]

(c) $\$ 8,000-\$ 9,999$

(j) $\square$ [10]

(k) $\square$ [11]

(d) $\$ 10,000$ - $\$ 11,999$

(I) $\square$ [12]

(m) $\square$ [13]

(e) $\$ 12,000-\$ 13,999$

(n) $\square$ [14]

(o) $\square$ [15]

(f) $\$ 14,000-\$ 15,999$

(p) $\square$ [16]

Don't Know..... $\square$ [98]

(g) $\$ 16,000 \cdot \$ 17,999$

Refused ...... $\square$ [96]

(h) $\$ 18,000 \cdot \$ 19,999$

(i) $\$ 20,000 \cdot \$ 24,999$

(j) $\$ 25,000 \cdot \$ 29,999$

(k) $\$ 30,000-\$ 34,999$

(l) $\$ 35,000 \cdot \$ 39,999$

(m) $\$ 40,000 \cdot \$ 49,999$

(n) $\$ 50,000 \cdot \$ 59,999$

(o) $\$ 60,000 \cdot \$ 74,999$

(p) $\$ 75,000$ OR OVER

IDA ISM PAGE 42 
135. We may have covered some of these points before, but just to be sure, please look at Exhibit \#135 and indicate whether these fuels are used for these purposes in your household.

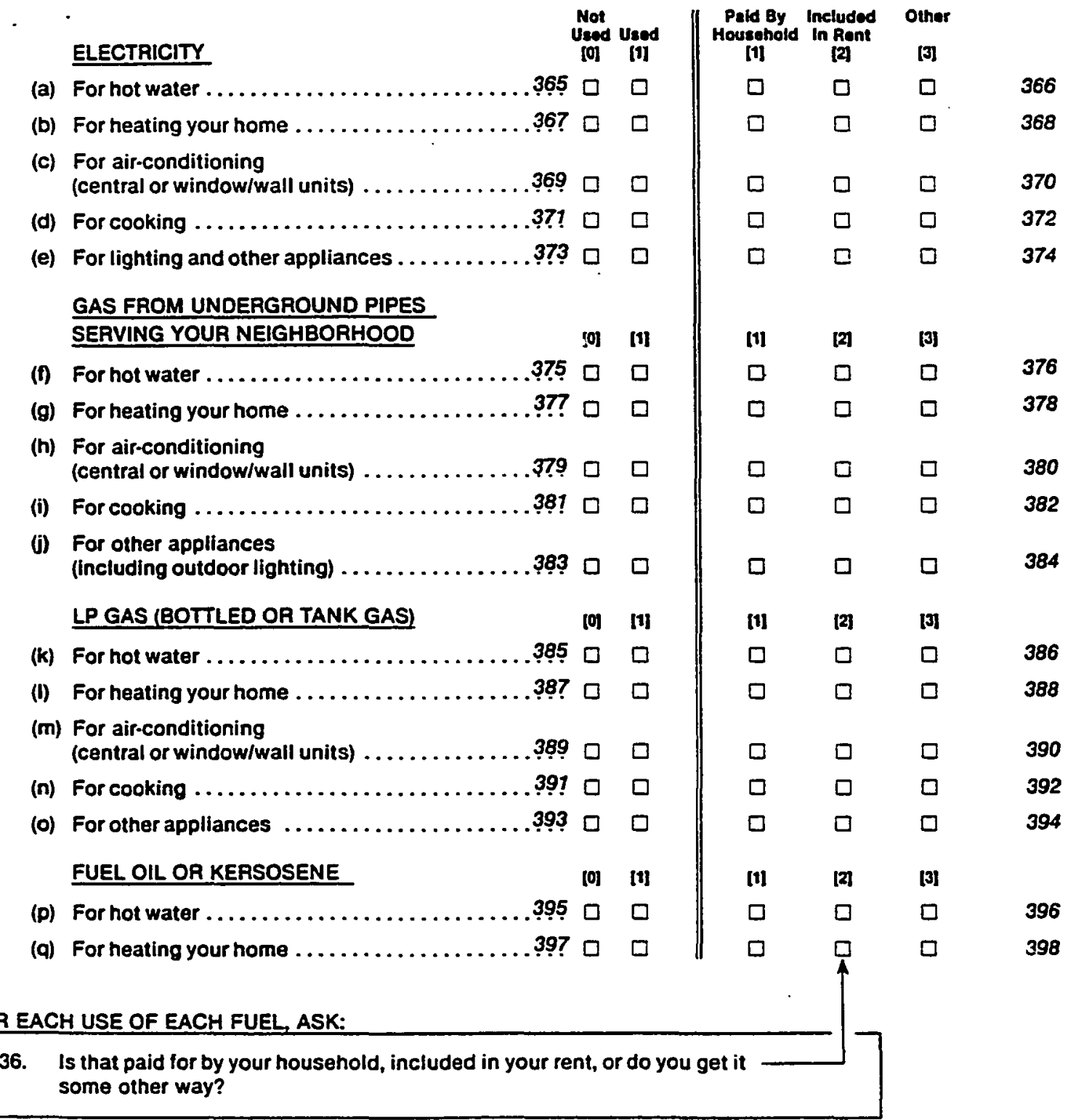


IF UNDERGROUND GAS IS NOT USED, ASK:

137. Is gas from underground pipes available in this neighborhood?

$$
\begin{array}{lll}
{[0]} & \square & \text { No } \\
{[1]} & \square & \text { Yes } \\
{[8]} & \square & \text { Don't Know }
\end{array}
$$

\section{IF ANY FUEL BILLS ARE PAID BY HOUSEHOLD, ASK:}

138. Is your electricity billed on a "budget" basis? That is, is your electricity bill the same each month?

$$
\begin{array}{lll}
{[0]} & \square & \text { No (varies) } \\
{[1]} & \square & \text { Yes (same) } \\
{[7]} & \square & \text { Not Applicable }
\end{array}
$$

IF “NATURAL GAS" USED, ASK:

139. Is your natural gas billed on a "budget" basis?

$$
\begin{array}{lll}
{[0]} & \square & \text { No (varies) } \\
{[1]} & \square & \text { Yes (same) } \\
{[7]} & \square & \text { Not Applicable }
\end{array}
$$




\title{
PACIFIC NORTHWEST \\ RESIDENTIAL ENERGY CONSUMPTION SURVEY
}

\author{
AUTHORIZATION FORM FOR \\ UTILITY CONSUMPTION INFORMATION
}

Department of Energy

Bonneville Power Administration

P.O. Box 3621

Portiand, Oregon 97208

I hereby give permission to the company or companies listed below to provide information to Louis Harris and Associates for use in connection with their survey for the Bonneville Power Administration.

This authorization covers the quantity and price of electricity and natural gas purchased by my household beginning September 1981 and continuing through December 1982. Companies are authorized to provide this information by monthly or bimonthly periods, whichever applies.

A photocopy of this authorization may be accepted with the same authority as the original.

I understand that this energy quantity and price information will be linked with my answers to the interview questions. $I$ also understand that the energy information and interview answers may be provided to my local electric utility or natural gas company (where applicable), and that these companies may be able to identify me. However, this information shall be used for statistical purposes only. My name will never be published as a participant in this survey and I will never be contacted for advertising or promotional purposes. The electric utility or natural gas company agrees to limit access to this information and to treat me no differently than they do all of their residential customers.

I understand that I may be contacted in future surveys conducted for the Bonneville Power Administration. My participation in those future surveys will be completely voluntary and independent of my participation in this survey.

I have read and understood the above statements and agree to their provisions.

Date: Signature:

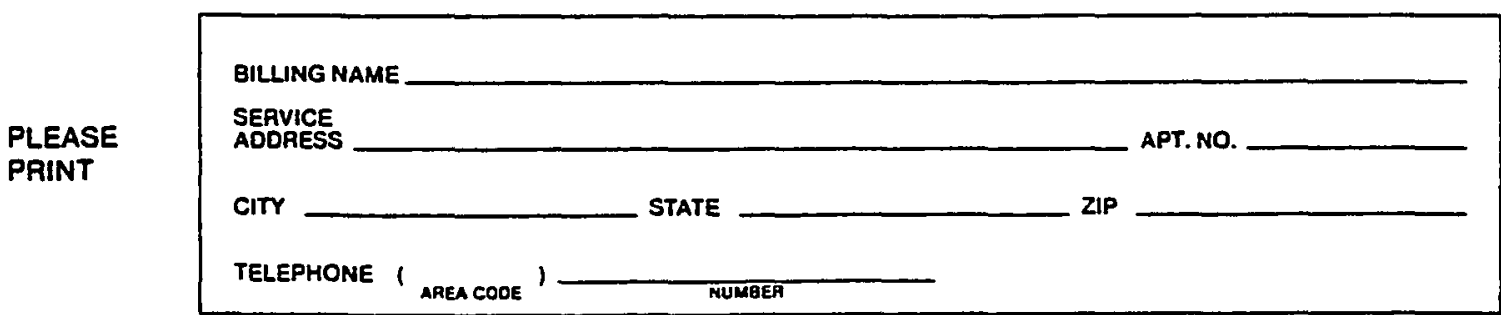

PLEASE COMPLETE ONE BLOCK BELOW FOR EACH FUEL USED BY YOUR HOUSEHOLD.

ELECTRICITY
\begin{tabular}{|l|l|l|}
\hline PRINT FULL NAME OF ELECTAIC COMPANY & ACCOUNT MUMBER \\
\hline PRINT FULL NAME OF GAS COMPANY & ACCOUNT NUMBER \\
FATURAL GAS \\
GROUNO PIPES
\end{tabular}

IDA ISAO PAGE 40 


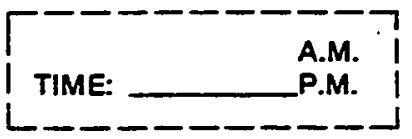

VAR

0-1. In order to complete this interview, there are two other tasks that 1 would like to perform. First, would you mind if I measure the temperature of the "hot" tap water in your home? I can do this at any sink which has a "hot" water tap.

$$
\begin{aligned}
& \text { RECORD: Temperature of hot water } \\
& \qquad \begin{aligned}
\text { [997] } & \square \text { No hot water } \\
{[996] } & \square \text { Refused }
\end{aligned}
\end{aligned}
$$

0-2. Has any hot water been used in the last hour for things like clothes washing, dishwashing, bathing or other tasks which use large amounts of hot water?

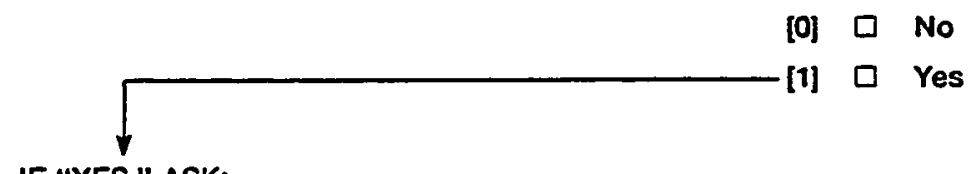

IF "YES," ASK:

0-3. What was it used for?

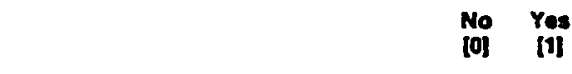

a. Clothes washing........ $\square \quad \square$

b. Dish washing ......... $\square \quad \square \quad 405$

c. Bathing ............. $\square \quad \square \quad 406$

d. Other............. $\square \quad \square \quad 407$

0-4. Finally, one of the most important things affecting people's energy use is the size of their home. With your permission, I would like to measure your home. Your assistance in this task would be appreciated.

In order to accurately measure your home, I would like to know about a few of the features of your home.

0.5. First, how many floors are used as living quarters?

$\begin{array}{llllll}\text { [01] } & \square & \text { One floor } & \text { [06] } & \square & \text { More than } 3 \text { floors } \\ \text { [02] } \square & 11 / 2 \text { floors } & {[11]} & \square & \text { Split level-2 levels } \\ \text { [03] } \square & 2 \text { floors } & {[12]} & \square & \text { Split level-3 levels } \\ \text { [04] } \square & 21 / 2 \text { floors } & {[13]} & \square & \text { Split level-More than } 3 \text { levels } \\ \text { 105] } \square & 3 \text { floors } & {[21]} & \square & \text { Other-Describe: }\end{array}$


0.6. How many floors that are used as living quarters are completely below ground?
[0] $\square$ None
[2] $\square$ One floor
[1] $\square$ Partial floor
[3] $\square$ More than one floor

0-7. How many floors that are used as living quarters are partially below ground-for example, a daylight basement?
[0] $\square$ None
[2] $\square$ One floor
[1] $\square$ Partial floor
[3] $\square$ More than one floor

IF "BUILDING WITH 2 OR MORE UNITS," ASK:

0-8 On what floor is this unit located, starting with the first floor which contains dwelling units?

Floor Number

0-9. What type of material is primarily used on the outside walls of this house (building)?

IF TWO MATERIALS ARE USED ABOUT THE SAME AMOUNT, RECORD BOTH MATERIALS.

\begin{tabular}{|c|c|c|}
\hline $\begin{array}{c}\text { Not } \\
\text { Used } \\
\text { [0] }\end{array}$ & $\begin{array}{l}\text { Used } \\
\text { [1] }\end{array}$ & \\
\hline$\square$ & $\square$ & Wood \\
\hline$\square$ & $\square$ & Brick \\
\hline$\square$ & $\square$ & Stone \\
\hline$\square$ & $\square$ & Concrete \\
\hline$\square$ & $\square$ & Stucco \\
\hline$\square$ & $\square$ & Aluminum Siding \\
\hline$\square$ & $\square$ & Steel Siding \\
\hline$\square$ & $\square$ & Composition Siding (Asbestos, Vinyl) \\
\hline$\square$ & $\square$ & Glass \\
\hline$\square$ & $\square$ & Other (Describe: \\
\hline
\end{tabular}


INTERVIEWER INSTRUCTIONS:

In general, measure all parts of the housing unit which are used as year-round living quarters.

1-- - - - - - - - - - - - - - - - - - - - - - - - - -

- Basements

Include basements in one-family houses if at least some portion is heated (refer to 0.37).

Include basement space in buildings with 2 to 4 housing units, if at least some portion is heated and it is for the exclusive use of the household interviewed.

Exclude basements in one-family houses and buildings with 2 to 4 units if no portion of the basement is heated.

Exclude basements in buildings with 5 or more units.

Exclude crawl spaces.

- Attics

include attics if heated or finished. Check appropriate box on corresponding diagram (refer to Q-17/18).

Exclude attics if unheated and also unfinished.

- Garages, sheds or barns

Include garages only if attached to house, enclosed from the weather and heated.

Exclude garages, sheds, or barns if not attached to house or open to the weather or unheated.

- Porches

Include porches if enclosed from the weather and heated.

Exclude porches if open to the weather or unheated.

- Buildings with 2 or more housing units

Measure only the space used exclusively by the interviewed household. Do not measure the entire building. Do not measure entryways, hallways, or any other areas which are not for the exclusive use of the interviewed household.

- - - - - - - - - - - - - - - - - - - - - - - - - - - - - - USE BACKS OF MEASUREMENT PAGES FOR ADDITIONAL SPACE AS NEEDED, FOR SKETCHES AND MEASUREMENTS. 
START HERE,

if this household has a basement or cellar (see instruction on

facing page for

basements and

cellars)
RECORD MEASUREMENTS ON DIAGRAM TO NEAREST FOOT

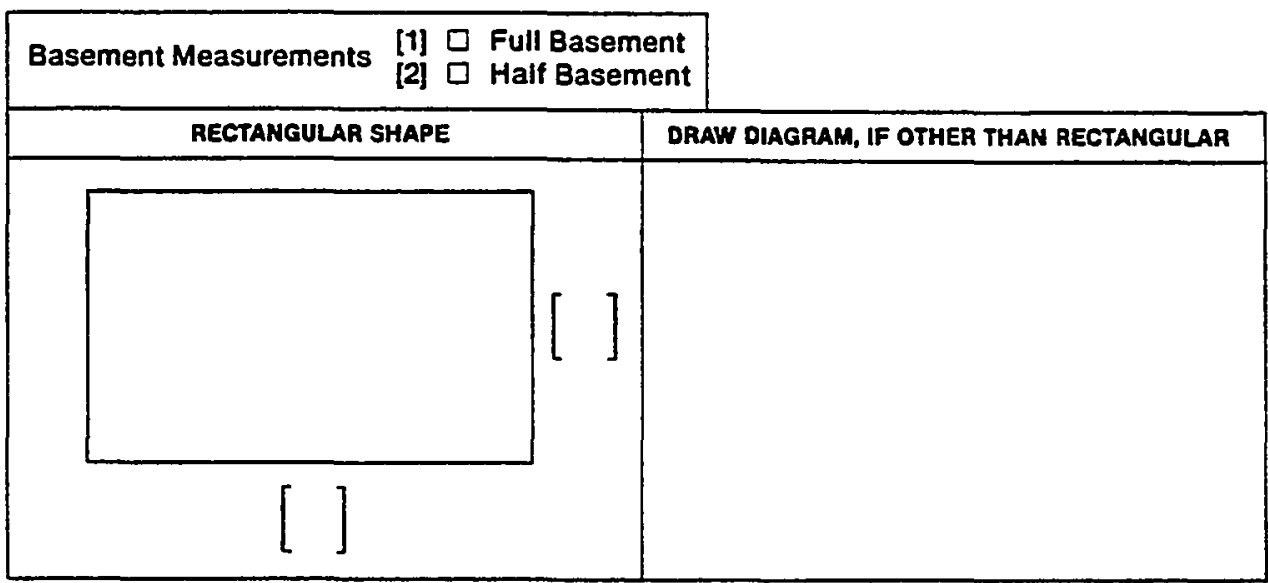

\section{START HERE}

If this household does not have a besement or cellar

\section{First Story Measurements [1] $\square$ Full Story \\ [2] $\square$ Half Story}

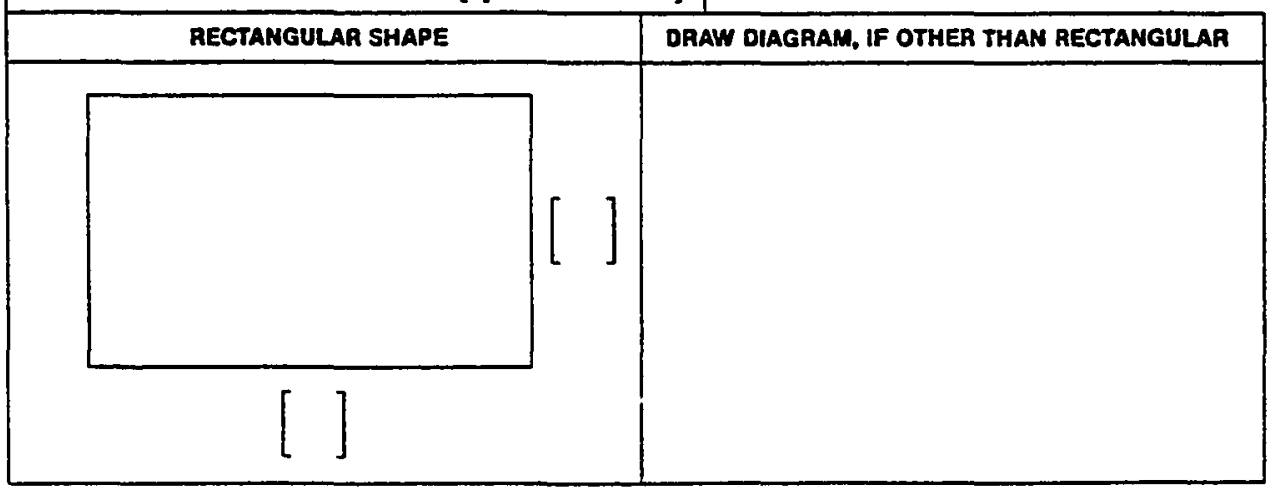

CONTINUE ON PAGE 51

FOR SECOND AND

THIRD STORIES

FOR OFFICE USE ONLY

B

\begin{tabular}{|c|c|c|c|c|c|c|c|c|c|c|}
\hline $\begin{array}{c}\text { Full. } \\
\text { Hall }\end{array}$ & \multicolumn{2}{|c|}{ Unit A } & \multicolumn{2}{c|}{ Unit B } & \multicolumn{2}{c|}{ Unit C } & \multicolumn{2}{c|}{ Unit D } & Unit X & $\begin{array}{c}\text { of of } \\
\text { Units }\end{array}$ \\
\hline 422 & 423 & 424 & 425 & 426 & 427 & 428 & 429 & 430 & 431 & 432 \\
\hline & & & & & & & & & & \\
\hline 433 & 434 & 435 & 436 & 437 & 438 & 439 & 440 & 447 & 442 & 443 \\
\hline & & & & & & & & & & \\
\hline
\end{tabular}

IPA ISM PAGE SO 
IF NO SECOND OR THIRD STORY TO MEASURE, GO TO Q-R-1

RECORD MEASUREMENTS ON DIAGRAM TO NEAREST FOOT

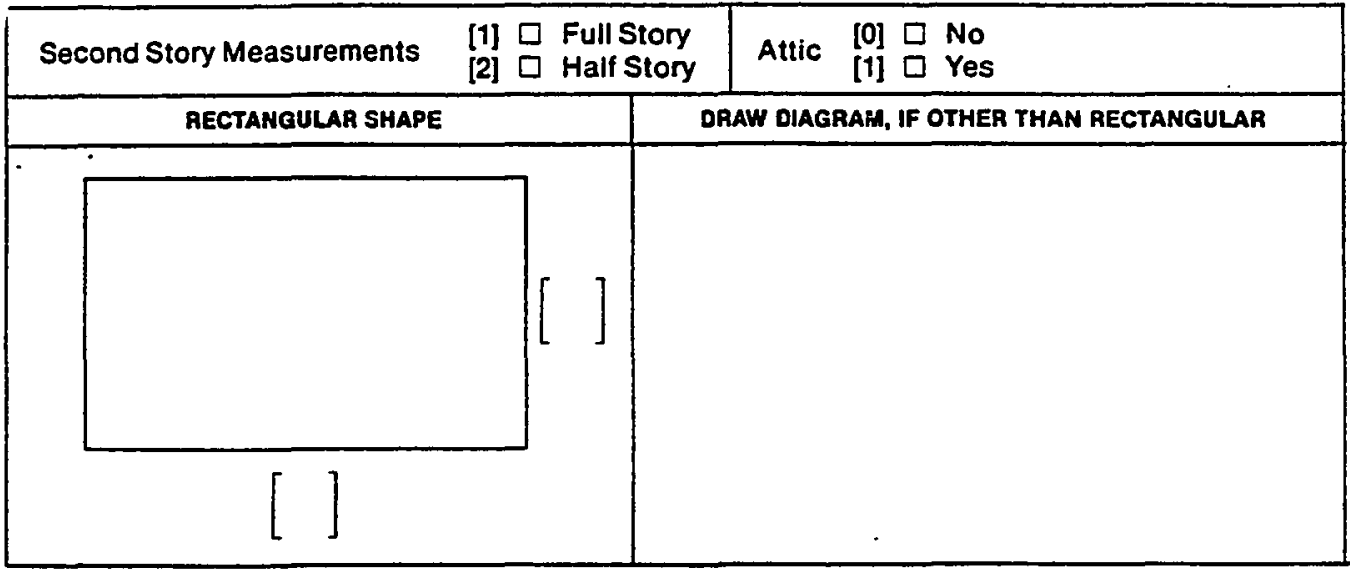

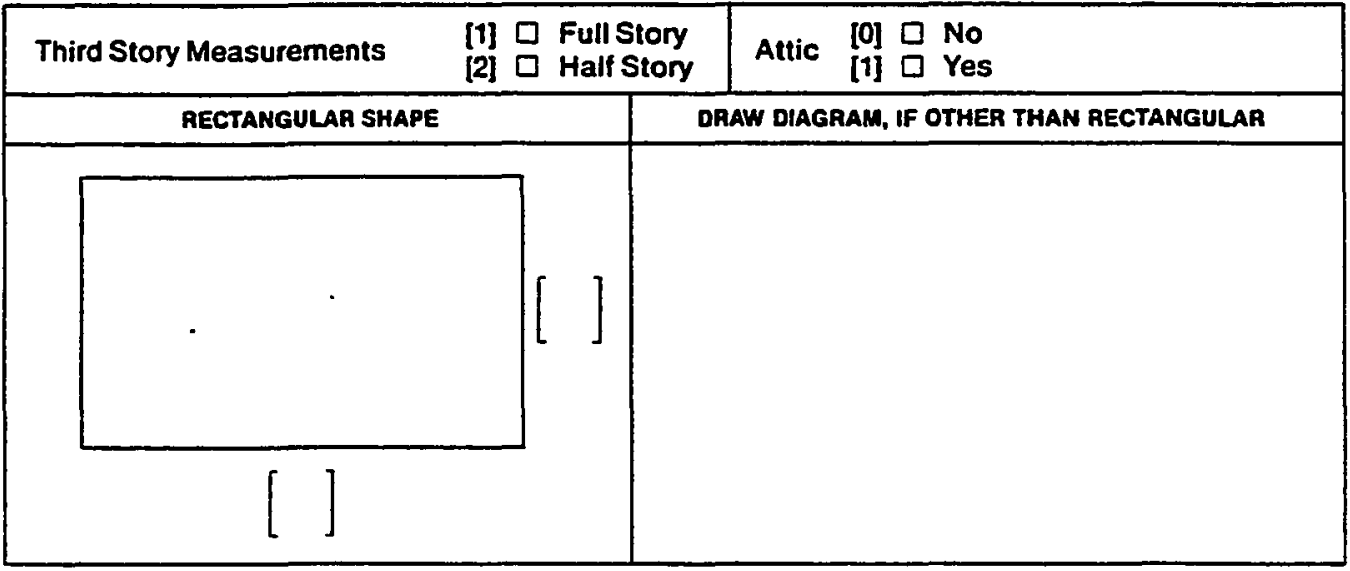

FOR OFFICE USE ONLY

2

\begin{tabular}{|c|c||c|c|c|c|c|c|c|c|c||c|}
\hline $\begin{array}{c}\text { Fult. } \\
\text { Halt }\end{array}$ & Attic & \multicolumn{2}{|c|}{ Unit A } & \multicolumn{2}{|c|}{ Unit B } & \multicolumn{2}{|c|}{ Unit C } & \multicolumn{2}{|c|}{ Unit D } & Unit X & $\begin{array}{c}\text { of of } \\
\text { Units }\end{array}$ \\
\hline 444 & 445 & 446 & 447 & 448 & 449 & 450 & 451 & 452 & 453 & 454 & 455 \\
\hline & & & & & & & & & & & \\
\hline 456 & 457 & 458 & 459 & 460 & 461 & 462 & 463 & 464 & 465 & 466 & 467 \\
\hline & & & & & & & & & & & \\
\hline
\end{tabular}

TOTALS

\begin{tabular}{|c|c|c|c|c||c|}
\hline Besomont & First & Second & Third & Mise. & Grand \\
\hline 468 & 469 & 470 & 471 & 472 & 473 \\
\hline & & & & & \\
\hline
\end{tabular}


INTERVIEWER REPORT ON MEASUREMENT OF DWELLING UNIT:

R-1. HOW WERE MEASUREMENTS OBTAINED?

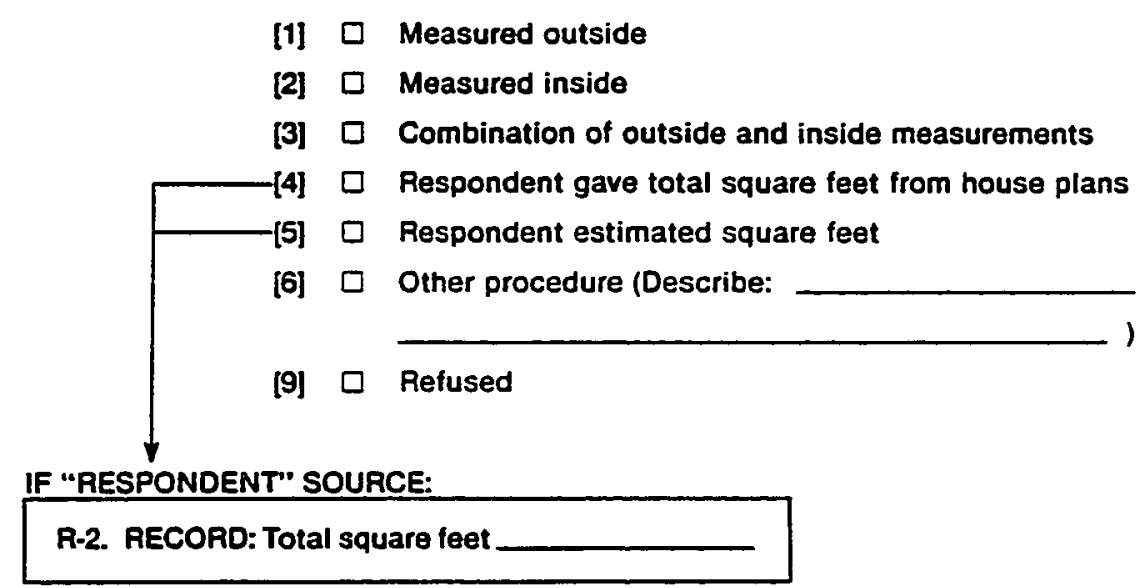

R-3. WHAT PROBLEMS, IF ANY, DID YOU HAVE IN MEASUAING THIS DWELLING UNIT?

R-4. WHAT EFFECT, IF ANY, DID THESE PROBLEMS HAVE ON THE ACCURACY OF YOUR MEASUREMENTS?

DPA ISEN PAGE 52 
DATE:

VAR

Day

475

476

TIME:

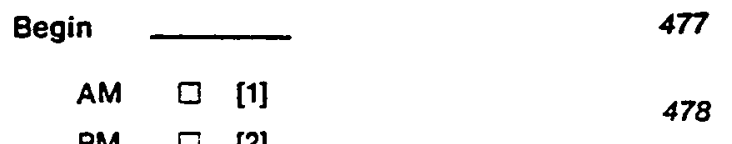

End Interview/

A:A $\square$ [1]

PM $\square$ [2]

480

End Measure

481

AM $\square \quad$ [1]

PM $\square \quad$ [2]

482

INTERVIEWER:

ID \#

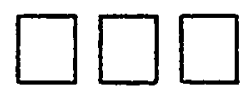

483

Signature 


\section{BILLING HISTORY DATA}

The billing history data file is divided into two parts. The first part of the data file contains the electricity billing histories data, and the natural gas billing data is contained in the second part of the file.

Each part of the file contains the basic data base and case identification information, and is directly comparable to the identification information contained in the interview data file.

The format of the two billing history portions of the file are directly comparable to one another. To assist the user, the labeling and numbering schemes for the variables are equivalent except for a preceding " $E$ " (electricity) or "G" (natural gas). Thus "EVAR701" denotes the "Beginning Date" for electricity billing period 1, while "GVAR701" denotes the "Beginning Date" for gas billing period 1 .

To conserve space, a generic list of the variables is included here; the preceding " $E$ " or " $G$ " has been onitted from the Referant or "VAR Name". Please note that in using the data, it is necessary to include these prefixes. 


\section{VARIABLE LIST}

Referant (VAR Name)

ID

SOURCE

DBNUM

EDITION

DBTYPE

DATE

SAMPTI:

UTIL

GEO

MRR

SITE

UBILL

BILLPD
Definition

Serial case identification number

Data Base Number

Sample Point Number

Fuel and Unit Identification

$$
\begin{aligned}
& 1=\text { Electricity, KWh } \\
& 2=\text { Gas, Therms } \\
& 3=\text { Gas, BTU }
\end{aligned}
$$

$$
\begin{aligned}
\text { Billing Period } \\
1=\text { Monthly } \\
2=\text { Bimonthly } \\
3=\text { Tri-monthly } \\
4=\text { Quarterly } \\
5=\text { Semi-annually } \\
6=\text { Annualiy }
\end{aligned}
$$


Referant (VAR Name)

VAR701 to VAR720

VAR721 to VAR740

VAR741 to VAR760

VAR761

VAR762

VAR763

VAR764

VAR765

VAR766

\section{Definition}

Beginning Date, Billing Period 1 to Period 20

Consumption, Billing Period 1 to Period 20

Cost, Billing Period 1 to Period 20

End Date, Last Valid Billing Period

Whether Data Provided

1 = Data Present

2 = Invalid or no waiver

3 = Data not available

4 = Other

Whether Billed on a Budget Basis

1 = Budget Basis

$2=$ Billed as Budget, Actual Readings Recorded

3 = Actual Basis

Whether Utility has Provided an Energy Audit

1 = Audit Provided

2 = Audit Requested, not yet Provided

3 = No Data Available in Utility Records

4 = No Audit Provided or Requested

Type of Assistance

1 = Zero Interest Loan From Utility

2 = BPA Buyback Program Participant

3 = Water Heater Wrap

4 = Grant

5 = BPA Weatherization Program Participant

6 = Other Weatherization Assistance

7 = No Assistance Provided

8 = Other

Servicing Gas Company 


\section{NOAA WEATHER DATA}

Heating and cooling degree days have been calculated using National Oceanographic and Atmospheric Administration (NOAA) data for climatological divisions (CD). The degree days have been calculated separately for electricity billing periods and natural gas billing periods, and are divided into two distinct computer files.

For each fuel, heating degree day (HDD) and cooling degree day (CDD) data are provided for each billing period for which consumption data are available. The applicable time frame (September 1981 through May 1983) has been divided into 20 such periods.

The data for each case are divided into four parts, each corresponding to a pair of HDD/CDD Bases, as follows:

Heating Degree Days

$\begin{array}{lll}1 . & 55 & 65 \\ 2 . & 60 & 70 \\ 3 . & 65 & 75 \\ 4 . & 70 & 80\end{array}$

Each record contains the serial identification code, the CD code, and the relevant $\mathrm{HDD}$ and $\mathrm{CDD}$ base temperatures, in addition to the actual HDD and CDD data for each applicable fuel billing period.

The format of the two files is identical, as is the format of each of the four records per case. As with the billing history data definitions, the labels for the electricity billing perlods weather data employ a preceding " $E$ " while the labels for the natural gas weather data employ a preceding "G".

Referant (VAR Name)

ID

CD

HDD1B

CDD1B

HDD1001 - HDD1020

$\operatorname{CDD} 1001-\operatorname{CDD} 1020$

\section{Definition}

Serial case identification number

NOAA Climatological Division Code

Heating Degree Day Base, 非 (55 -degree F)

Cooling Degree Day Base, \#1 (65 degree F)

HDD Data for Base \#1 for all Billing Periods

CDD Data for Base \#1 for all Billing Periods

This information is then repeated for each of the four degree day bases. 
APPENDIX IV

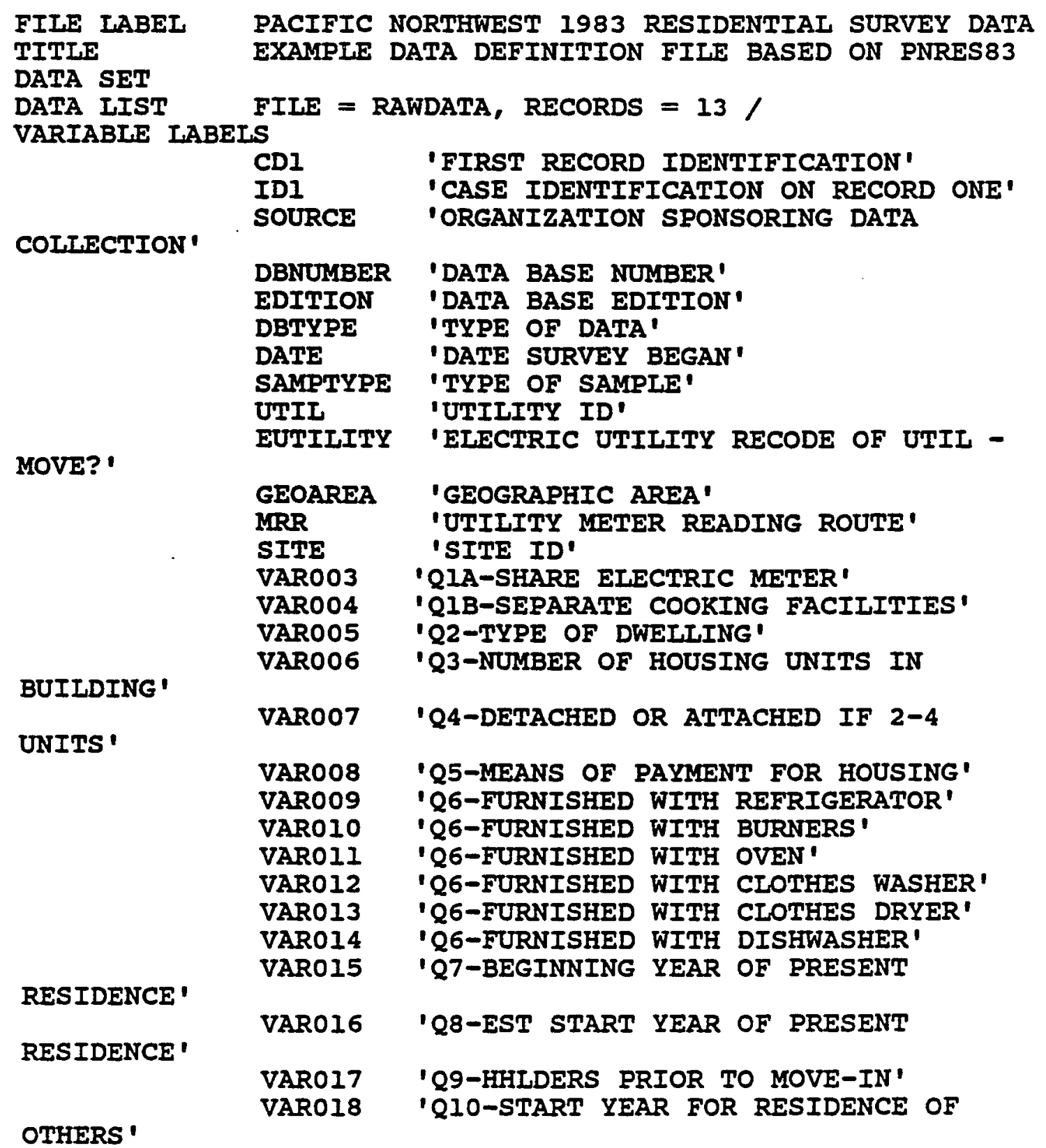


OTHERS '

RESIDENCE'

RENOVATED'

EATING'

EATING'

ROOMS '

POILUTION '
VAR019

VARO2O

VARO21

VAR022

VAR023

VAR024

VAR025

CD2

ID2

VAR026

VAR027

VAR028

VAR029

VAR0 30

VAR031

VAR032

VAR033

VAR034

VAR035

VAR036

VAR037

VAR038

VAR039

VAR040

VAR04 1

VAR042

VAR0 43

VAR044

VAR045

VAR04 6

VAR047

VAR048

VAR049

VAR050

VAR05I

VAR052

VAR053

VAR054

VAR055

VAR056

VAR057

VARO58

VAR059

VAR0 60

VAR061
'Q11-EST YEAR FOR RESIDENCE OF

'Q12-IF 1981-1983 THEN YEAR'

'QI2-BEGINNING MONTH OF PRESENT

'Q13-NEW HOUSE WHEN FIRST OCCUPIED'

'Q14-YEAR DWEILING BUILT'

'Q15-YEAR DWELLING BUILT-ESTIM'

'Q16-DWELIING SUBSTANTIALLY

' CARD NUMBER 2 '

'SERIAL CASE ID2'

' Q17-\# OF ROOMS-IIVING ROOM'

'Q18-NO HEAT-\# LIVING ROOMS'

'Q17-\# OF ROOMS-DINING'

'Q18-NO HEAT-\# DINING ROOM'

'Q17-\# OF ROOMS-RITCHEN WITH EATING'

'Q18-NO HEAT-\# KITCHEN WITH EATING'

'Q17-\# OF ROOMS-KITCHEN WITHOUT

'Q18-NO HEAT-\# KITCHEN WITHOUT

'Q17-\# OF ROOMS-BEDROOM'

'Q18-NO HEAT-\# BEDROOM'

'Q17-\# OF ROOMS-DEN'

'Q18-NO HEAT-\# DEN'

'Q17-\# OF ROOMS-FAMILY ROOM'

'Q18-NO HEAT-\# FAMILY ROOM'

'Q17-\# OF ROOMS-UTIIITY ROOM'

'Q18-NO HEAT-\# UTILITY ROOM'

'Q17-\# OF ROOMS-OTHER SMALL ROOMS'

'Q18-NO HEAT-\# OTHER SMALI ROOMS'

'Q17-\# OF ROOMS-OTHER LARGE ROOMS'

'Q18-NO HEAT-\# OTHER LARGE ROOMS'

'Q17-NUMBER OF ROOMS IN IIVING SPACE'

'Q18-NUMBER OF CLOSED OFF-UNHEATED

'Q18A-ANY ROOMS NOT HEATED'

'Q19-ENERGY EFFICIENCY OF HOME'

'Q20-THOUGHTS ON ENVIRONMENTAL

'Q20-THOUGHTS ON COST OF ENERGY'

'Q20-THOUGHTS ON UNEMPLOYMENT'

' Q20-THOUGHTS ON SCARCITY OF ENERGY'

'Q20-THOUGHTS ON INFLATION'

'Q20-THOUGHTS ON CRIME'

'Q21-COST PROBLEM - ELECTRICITY'

'Q21-SCARCITY PROBIEM - ELECTRICITY'

'Q2I-COST PROBLEM - NATURAL GAS'

'Q21-SCARCITY PROBLEM - NATURAL GAS'

'Q21-COST PROBLEM - HEATING OII'

'Q21-SCARCITY PROBLEM - HEATING OIL' 


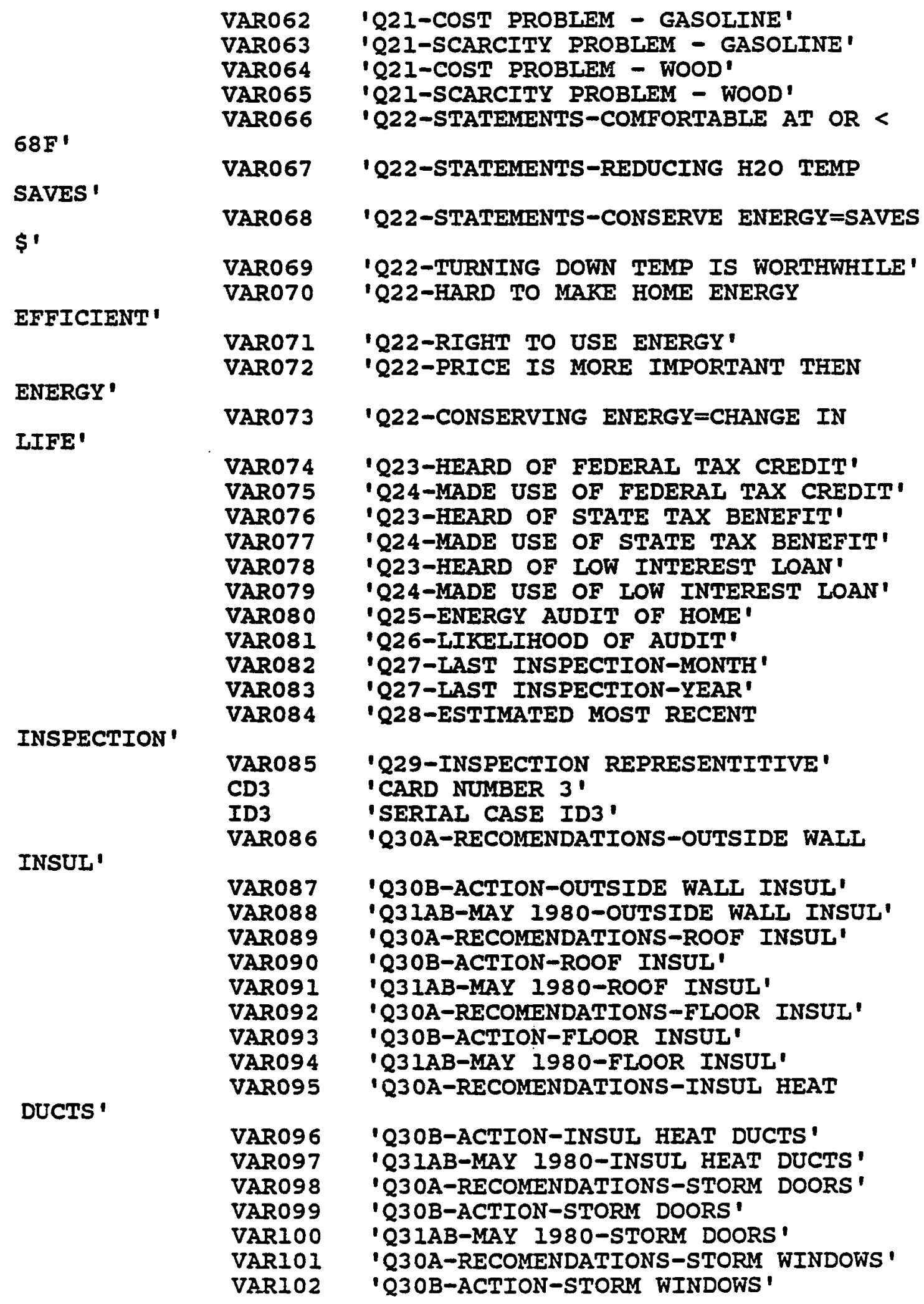




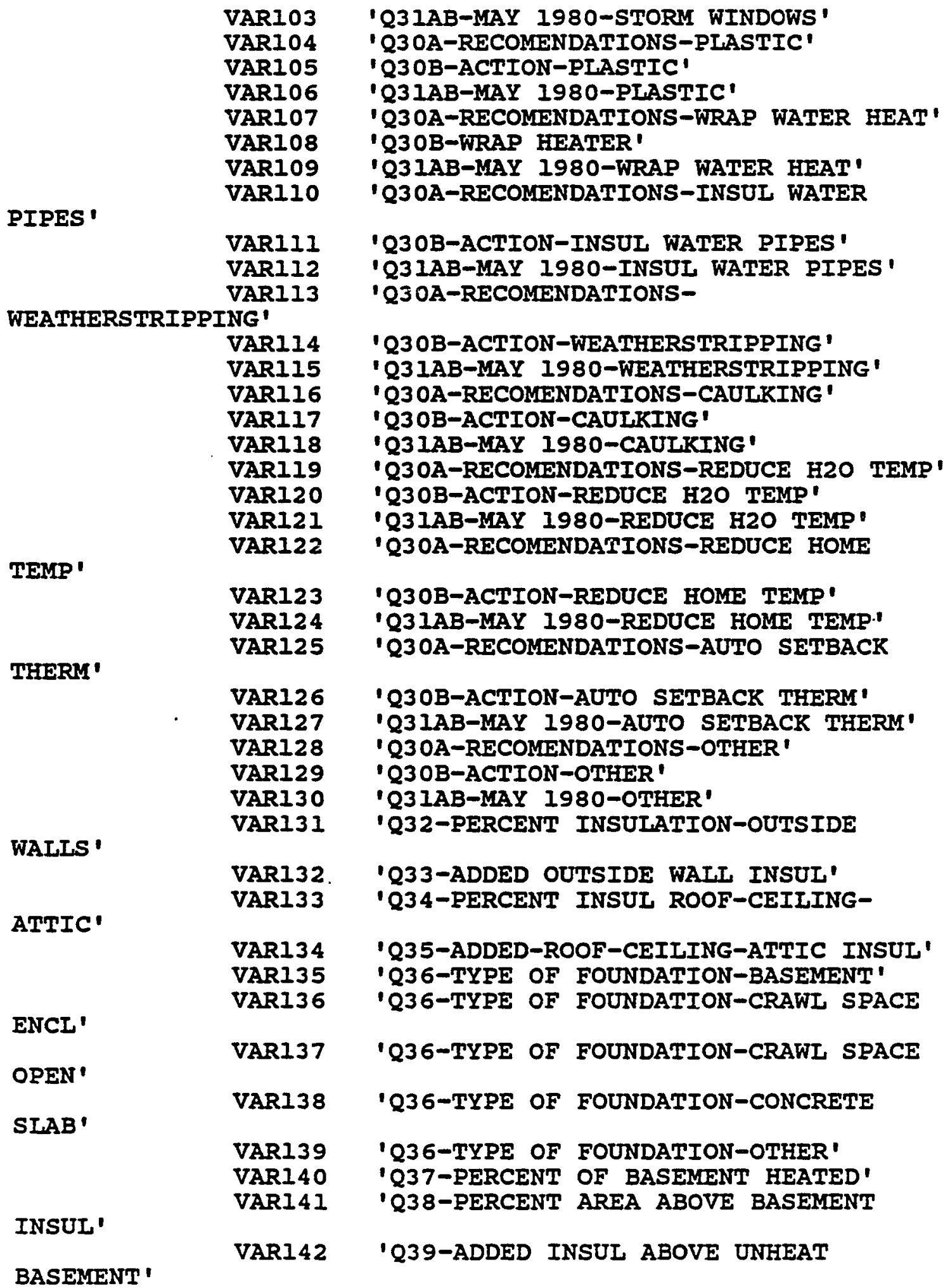


INSUL '

AREA !

GLASS '

GAS '

ELEC

WOOD'

PAST YR'

OII'.

KEROSENE'

GEOTHERMAL'
VAR143 'Q40-PERCENT HEATED WALIS-BASEMENT

VAR144

CD4

ID4

VAR145

VARI4 6

VARI47

VARI48

VARI49

VAR150

VAR151

VAR152

VAR153

VAR154

VAR155

VARI56

VARI57

VARI58

VAR159

VARI 60

VAR161

VARI62

VARI63

VARI64

VARI 65

VARI 66

VARI 67

VARI68

VARI69

VARI70

VARI71

VARI72

VARI73

VAR174

VARI75

VARI76

VARI77

VARI78

VAR179

VAR180

VARI81

'Q41-ADDED INSULATION BASEMENT WALLS'

' CARD NUMBER 4'

' SERIAI CASE ID 4 '

'Q42-NUMBER OF DOORS TO UNHEATED

'Q43-NUMBER OF OUTSIDE STORM DOORS' 'Q44-NUMBER OF SIIDING GLASS DOORS'

'Q45-DOORS WITH INSULATING GLASS'

'Q46-WINDOWS-INSULATED OR THERMOPANE'

'Q46-STORM WINDOWS'

'Q46-PLASTIC OVER WINDOWS'

'Q46-OTHER'

'Q47-PERCENT INSULATED-THERMOPANE

'Q47-PERCENT STORM WINDOWS'

'Q47-PERCENT PIASTIC OVER WINDOWS'

'Q47-PERCENT OTHER'

'Q48-TYPE OF FUEL USED MOST'

'Q49-EQUIP USED MOST TO HEAT HOME-

'Q50-EQUIP USED MOST TO HEAT HOME-

'Q51-EQUIP USED MOST TO HEAT HOME-

'Q52-EQUIPMENT INSTALIED ON FP\#l'

'Q52-EQUIPMENT INSTALIED ON FP\#2'

'Q53-OWN OR SHARE HEATING SYSTEM'

'Q54-HEATING SYSTEM CHANGE DURING

'Q55-MONTH OF CHANGE'

'Q55-YEAR OF CHANGE'

'Q56-FORMER TYPE OF HEATING FUEL'

'Q57-HEAT EQUIP ALREADY INSTALLED'

'Q58-YEAR EQUIPMENT INSTALIED'

' Q59-ESTM YEAR HEAT EQUIP INSTALIED'

'Q60-USE OF OTHER HEATING FUELS'

'Q61-ADDITIONAL HEATING FUEL-WOOD'

'Q61-ADDITIONAL HEATING FUEL-ELEC'

'Q61-ADDITIONAL HEATING FUEL-GAS'

'Q61-ADDITIONAL HEATING FUEL-FUEI

'Q61-ADDITIONAI HEATING FUEL-PROPANE'

'Q61-ADDITIONAT HEATING FUEL-

'Q6I-ADDITIONAL HEATING FUEL-COAL'

'Q6I-ADDITIONAL HEATING FUEL-SOLAR'

'Q61-ADDITIONAL HEATING FUEL-

'Q61-ADDITIONAL HEATING FUEL-OTHER' 
KEROSENE '

$\mathbf{Y R}^{\prime}$

$Y^{\prime}{ }^{\prime}$

YR'

SYSTEM '

HEATED'

1981'
VAR182

VAR183

VARI84

VAR185

VAR186

VARI87

VAR188

VAR189

VAR190

VARI91

VARI92

CD5

ID5

VAR193

VAR194

VAR195

VARI96

VAR197

VAR198

VAR199

VAR200

VAR201

VAR202

VAR203

VAR204

VAR2 05

VAR2 06

VAR207

VAR208

VAR209

VAR210

VAR211

VAR2 12

VAR2 I3

VAR2 I4

VAR2 15

VAR2 16

VAR2 17

VAR2 18

VAR2 19

VAR2 20

VAR22I

VAR2 22

VAR223

VAR224
'Q62-TYPE OF EQUIP GAS OII OR

'Q63-TYPE OF EQUIP IF ELECTRICITY'

'Q64-TYPE OF EQUIP IF WOOD'

'Q65-EQUIP FIREPLACE \#1'

'Q65-EQUIP FIREPLACE\#2'

'Q66-BURNED WOOD OR PRESTOLOGS PAST

'Q67-NUMBER OF LOGS BURNED IN IAST

'Q68-NUMBER OF CORDS BURNED IN LAST

'Q69-PERCENT OF WOOD PURCHASED'

'Q70-THERMOSTAT ADJUSTS HEATING TEMP'

'Q71-TYPE OF THERMOSTAT ON HEATING

' CARD NUMBer 5'

'SERIAL CASE ID 5'

'Q72A-TEMP OF HOUSE-SOMEONE HOME'

'Q72-LOW TEMP-SOMEONE HOHE'

'Q72-HIGH TEMP-SOMEONE HOME'

'Q72B-TEMP OF HOUSE-NO ONE HOME'

'Q72-LOW TEMP-NO ONE HOME'

'Q72-HIGH TEMP-NO ONE HOME'

'Q72C-TEMP OF HOUSE-SLEEPING HOURS'

'Q72-LOW TEMP-SIEEPING HOURS'

'Q72-HIGH TEMP-SLEEPING HOURS'

'Q73-SOMEONE HOME 9-5 AND HOUSE

'Q74-PERIODS OF NO USE SINCE SEPT

'Q75-PERIOD OF NO USE-SEPT 1981'

'Q75-PERIOD OF NO USE-OCT 1981'

'Q75-PERIOD OF NO USE-NOV 198I'

'Q75-PERIOD OF NO USE-DEC 1981'

'Q75-PERIOD OF NO USE-JAN 1982'

'Q75-PERIOD OF NO USE-FEB 1982'

'Q75-PERIOD OF NO USE-MARCH 1982'

'Q75-PERIOD OF NO USE-APRII 1982'

'Q75-PERIOD OF NO USE-MAY 1982'

'Q75-PERIOD OF NO USE-JUNE 1982'

'Q75-PERIOD OF NO USE-JULY 1982'

'Q75-PERIOD OF NO USE-AUG 1982'

'Q75-PERIOD OF NO USE-SEPT $1982^{\prime}$

'Q75-PERIOD OF NO USE-OCT $1982^{\prime}$

'Q75-PERIOD OF NO USE-NOV 1982'

'Q75-PERIOD OF NO USE-DEC 1982'

'Q75-PERIOD OF NO USE-JAN 1983'

'Q75-PERIOD OF NO USE-FEB 1983'

'Q75-PERIOD OF NO USE-MAR $1983^{\prime}$

'Q75-PERIOD OF NO USE-APRII 1983'

'Q75-PERIOD OF NO USE-MAY 1983' 


\begin{tabular}{|c|c|c|}
\hline & $\begin{array}{l}\text { VAR2 } 25 \\
\text { VAR2 } 26 \\
\text { VAR227 } \\
\text { VAR228 }\end{array}$ & $\begin{array}{l}\text { 'Q75-PERIOD OF NO USE-NOT KNOWN' } \\
\text { 'Q75-PERIOD OF NO USE-REFUSED ANSWER' } \\
\text { 'Q75-PERIOD OF NO USE-NA' } \\
\text { 'Q76-TYPE OF FUEL USED FOR HEATING }\end{array}$ \\
\hline WATER' & $\begin{array}{l}\text { AR2 } 29 \\
\text { AR2 } 30\end{array}$ & $\begin{array}{l}\text { 'Q77-SOURCE OF WATER HEATER' } \\
\text { 'Q78-HOT WATER EQUIP INSTALLED AT }\end{array}$ \\
\hline $\begin{array}{l}\text { MOVE IN' } \\
\text { ACTUAL' }\end{array}$ & VAR231 & 'Q79-HOT WATER EQUIP INSTALIED- \\
\hline & $\begin{array}{l}\text { VAR2 } 32 \\
\text { VAR233 }\end{array}$ & $\begin{array}{l}\text { 'Q80-HOT WATER EQUIP INSTALIEDD-ESTM' } \\
\text { ' Q81-WATER HTR IN HEATED OR UNHEATED }\end{array}$ \\
\hline $\begin{array}{l}\text { AREA' } \\
\text { INSULATION' }\end{array}$ & VAR234 & 'Q82-WATER HTR IS WRAPPED IN \\
\hline & $\begin{array}{l}\text { VAR2 } 35 \\
\text { VAR236 }\end{array}$ & $\begin{array}{l}\text { 'Q83-WRAP PROVIDED BY FUEL SUPPLIER' } \\
\text { 'Q84-OTHER TYPES OF FUEL FOR WATER }\end{array}$ \\
\hline HTR' & $\begin{array}{l}\text { VAR237 } \\
\text { VAR238 }\end{array}$ & $\begin{array}{l}\text { 'Q85-ADDITIONAI FUEL FOR WATER HTR' } \\
\text { ' Q86-NUMBER OF SHOWER FACIIITIES IN }\end{array}$ \\
\hline $\begin{array}{l}\text { HOME' } \\
\text { RESTRICTORS ' }\end{array}$ & VAR239 & 'Q87-\# OF SHOWERS WITH FLOW \\
\hline INSERTS ' & VAR240 & 'Q87-\# OF SHOWERS WITH LOW-FLOW \\
\hline $\begin{array}{l}\text { DEVICE' } \\
\text { DEVICE' }\end{array}$ & $\begin{array}{l}\text { VAR24I } \\
\text { VAR242 }\end{array}$ & $\begin{array}{l}\text { 'Q88-\# OF FAUCETS WITH LOW-FLOW } \\
\text { 'Q89-PERMANENTLY REMOVED LOW-FLOW }\end{array}$ \\
\hline & VAR243 & 'Q90-REASON FOR REMOVING LOW-FLOW \\
\hline & $\begin{array}{l}\text { CD6 } \\
\text { ID6 } \\
\text { VAR244 } \\
\text { VAR245 } \\
\text { VAR246 } \\
\text { VAR247 } \\
\text { VAR248 } \\
\text { VAR249 } \\
\text { VAR250 } \\
\text { VAR251 } \\
\text { VAR252 } \\
\text { VAR253 } \\
\text { VAR254 } \\
\text { VAR255 } \\
\text { VAR256 } \\
\text { VAR257 } \\
\text { VAR258 } \\
\text { VAR259 } \\
\text { VAR260 } \\
\text { VAR261 } \\
\text { VAR262 }\end{array}$ & $\begin{array}{l}\text { 'CARD NUMBER 6' } \\
\text { 'SERIAL CASE ID 6' } \\
\text { 'Q91-IS HOME AIR CONDITIONED' } \\
\text { 'Q92-TYPE OF AIR CONDITIONING SYSTEM' } \\
\text { 'Q93-NUMBER OF AC WALI UNITS IN HOME' } \\
\text { 'Q94-TYPE OF CENTRAL AC SYSTEM' } \\
\text { 'Q95-USE OF AC \#I' } \\
\text { 'Q95-USE OF AC\#2' } \\
\text { 'Q96-AC\#I INSTAIIED AT MOVE IN' } \\
\text { 'Q96-AC\#2 INSTAIIED AT MOVE IN' } \\
\text { 'Q97-AGE OF AIR CONDITIONER \#I' } \\
\text { 'Q97-AGE OF AIR CONDITIONER \#2' } \\
\text { 'Q98-NUMBER OF TELEVISIONS' } \\
\text { 'Q99-TEIEVISION \#I-COLOR OR BW' } \\
\text { 'Q99-TELEVISION \#2-COLOR OR BW' } \\
\text { 'Q100-TELEVISION \#I-AGE' } \\
\text { 'Q100-TELEVISION \#2-AGE' } \\
\text { 'Q101-TYPE OF FUEL-STOVE TOP BURNERS' } \\
\text { 'Q102-STOVE TOP BURNERS INSTALIED' } \\
\text { 'Q103-AGE OF STOVE TOP BURNERS' } \\
\text { 'Q104-\# OF OVENS USED FOR COOKING' }\end{array}$ \\
\hline
\end{tabular}


BURNER '

BURNER'

HOME '

REFRIGERATOR \#I'

REFRIGERATOR \#2'

VAR280

VAR281

VAR282

VAR283

VAR284

VAR285

VAR286

VAR2 87

VAR28 8

CD7

ID7

METER '

METER '

ELEMENT'

REGULARLY '
VAR289

VAR290

VAR29I

VAR292

VAR293

VAR294

VAR295

VAR296

VAR297

VAR298

VAR299

VAR300

VAR301

VAR302

VAR303
'Q105-TYPE OF FUEI FOR OVEN \# 1 '

'Q105-TYPE OF FUEL FOR OVEN \#2'

'Q106-IS OVEN \#I A MICROWAVE'

'Q106-IS OVEN \#2 A MICROWAVE'

'Q107-IS OVEN \#I SEPARATE FROM TOP

'Q107-IS OVEN \#2 SEPARATE FROM TOP

'Q108-OVEN NUMBER I INSTaLIED'

'QI08-OVEN NUMBER 2 INSTALIED'

'QI09-AGE OF OVEN NUMBER 1 '

'QI09-AGE OF OVEN NUMBER 2 '

'QI10-IS OVEN \#I SELF-CLEANING'

'QIIO-IS OVEN \#2 SELF-CLEANING'

'QIII-NUMBER OF REFRIGERATORS IN

'Q112-SIZE OF REFRIGERATOR NUMBER I' 'Q112-SIZE OF REFRIGERATOR NUMBER 2'

'Q113-IOCATION OF FREEZER-

'Q113-LOCATION OF FREEZER-

'Q114-IS REFRIGERATOR \#I FROST FREE'

'Q114-IS REFRIGERATOR \#2 FROST FREE'

'Q115-HOME HAS SEPARATE FOOD FREEZER'

'QII6-SIZE OF SEPARATE FREEZER \#I"

'QI16-SIZE OF SEPARATE FREEZER \#2'

'QII7-TYPE OF SEPARATE FREEZER \#I'

'QI17-TYPE OF SEPARATE FREEZER \#2'

'Q118-TYPE OF FREEZER \#1-FROST FREE'

'Q118-TYPE OF FREEZER \#2-FROST FREE'

' CARD NUMBER 7 '

'SERIAL CASE ID7'

'Q119-CLOTHES WASHING MACHINE-HOME

'Q119-ELECTRIC CLOTHES DRYER-HOME

'Q119-GAS CLOTHES DRYER-HOME METER'

'Q119-ELECTRIC DISHWASHER-HOME METER'

'Q120-DISHWASHER-SEPARATE HEATING

'Q120-DISHWASHER-ENERGY SAVER SWITCH'

'Q121-USE ENERGY SAVER SWITCH

'Q122-HEATING ELEMENT DISCONNECTED'

'Q123-HOME HAS SWIMIING POOL'

'Q123-HOME HAS HOT TUB'

'Q123-HOME HAS JACUZZI'

'Q124-FUEL USED TO HEAT POOL'

'Q124-FUEL USED TO HEAT HOT TUB'

'Q124-FUEL USED TO HEAT JACUZZI'

'Q125-NUMBER OF WATERBED HEATERS' 


METER '
METER'
METER'
METER'
METER'
METER '
METER '
METER '
METER '
METER '
METER'

VAR304

VAR305

VAR306

VAR307

VAR308

VAR309

VAR310

VAR3 II

VAR3 12

VAR3 13

VAR3 14

VAR3 15

VAR3 16

VAR3 17

CD8

ID8

VAR3 18

VAR319

VAR320

VAR32I

VAR322

VAR323

VAR324

VAR325

VAR326

VAR327

VAR328

VAR329

VAR3 30

VAR3 31

VAR3 32

VAR3 33

VAR3 34

VAR3 35

VAR3 36

VAR3 37

VAR3 38

VAR3 39

VAR3 40

VAR3 41

VAR3 42
'Q126-MEDICAL EQUIP ON HOUSEHOLD

'Q126-PHOTO EQUIP ON HOUSEFOLD METER'

'Q126-WOODWORKING EQUIP ON HOUSE

'Q126-ELEC CERAMIC KIIN ON HOUSE

'Q126-GAS CERAMIC KIIN ON HOUSE

'Q126-HOME COMPUTER ON HOUSEHOLD

'Q126-OFFICE EQUIP ON HOUSEHOLD

'Q126-WELDING EQUIP ON HOUSEHOLD

'Q126-GREENHOUSE LIGHTS ON HOUSE

Q126-IRRIGATION PUMPS ON HOUSEHOID

'Q126-ELEC WEII WATER PUMP ON HOUSE

'Q127-OTHER EQUIP ON ELEC OR GAS

'Q128-OTHER EQUIP ON EIECTRIC BILL'

'Q128-OTHER EQUIP ON GAS BILI'

- CARD NUMBER 8'

'SERIAL CASE ID 8 '

'Q129-TOTAL NUMBER OF RESIDENTS'

'Q130-PERSON \#1-RESPONDENT CODE'

'Q130-PERSON \#1-GENDER'

'Q130-PERSON \#1-ACTUAL AGE'

'Q130-PERSON \# 1-AGE CATEGORY'

'Q130-PERSON \#2-RESPONDENT CODE'

'Q130-PERSON \#2-GENDER'

'Q130-PERSON \#2-ACTUAI AGE'

'Q130-PERSON \#2-AGE CATEGORY'

'Q130-PERSON \# 3-RESPONDENT CODE'

'Q130-PERSON \#3-GENDER'

'Q130-PERSON \#3-ACTUAL AGE'

'Q130-PERSON \#3-AGE CATEGORY'

'Q130-PERSON \#4-RESPONDENT CODE'

'Q130-PERSON \#4-GENDER'

'Q130-PERSON \#4-ACTUAL AGE'

'Q130-PERSON \#4-AGE CATEGORY'

'Q130-PERSON \#5-RESPONDENT CODE'

'Q130-PERSON \#5-GENDER'

'Q130-PERSON \#5-ACTUAL AGE'

'Q130-PERSON \#5-AGE CATEGORY'

'Q130-PERSON \#6-RESPONDENT CODE'

'Q130-PERSON \#6-GENDER'

'Q130-PERSON \#6-ACTUAL AGE'

'Q130-PERSON \#6-AGE CATEGORY' 


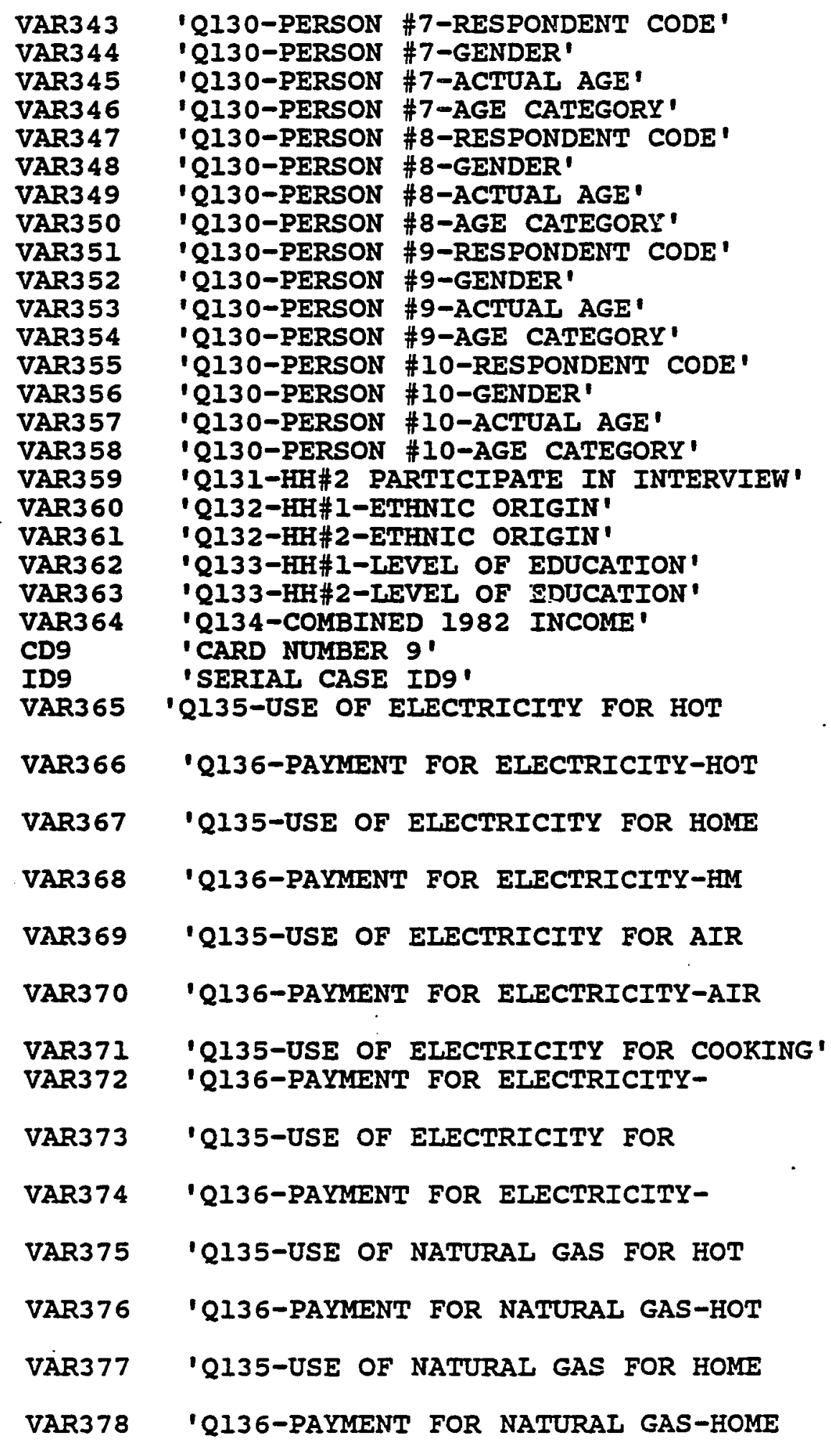


COND'

COND'

COOKING '

PURPOSES '

PURPOS '

HEATING '

HEATING '

CONDITION '

CONDITION '

PURPOSES '

PURPOSES '

HEATING '

GEATING '

CLOTHES '

DISHES '

QUARTERS '

GROUND'
VAR379 'Q135-USE OF NATURAL GAS FOR AIR

VAR380 'Q136-PAYMENT FOR NATURAI GAS-AIR

VAR381 'Q135-USE OF NATURAI GAS FOR COOKING'

VAR382 'Q136-PAYMENT FOR NATURAL GAS-

VAR383 'Q135-USE OF NATURAL GAS,MISC

VAR3 84

VAR385

VAR386

VAR387

VAR388

VAR389

VAR390

VAR391

VAR392

VAR393

VAR394

!

VAR395

VAR396

VAR397

VAR398

VAR399L

VAR400

VAR4 01

VAR402

VAR4 03

VAR404

VAR405

VAR406

VAR407

VAR4 08

VAR409

VAR4 10

VAR4 II

VAR4 12

VAR4 13

'Q136-PAYMENT FOR NATURAL GAS-MISC

'Q135-USE OF TANK GAS FOR HOT WATER'

'Q136-PAYMENT OF TANK GAS-HOT WATER'

'Q135-USE OF TANK GAS FOR HOME

'Q136-PAYMENT FOR TANR GAS-HOME

'Q135-USE OF TANK GAS FOR AIR

'Q136-PAYMENT FOR TANR GAS-AIR

'Q135-USE OF TANK GAS FOR COOKING'

'Q136-PAYMENT FOR TANK GAS-COOKING'

'Q135-USE OF TANK GAS FOR MISC

'Q136-PAYMENT FOR TANK GAS-MISC

'Q135-USE OF FUEL OIL FOR HOT WATER'

'Q136-PAYMENT FOR FUEL OIL-HOT WATER'

'Q135-USE OF FUEL OIL FOR HOME

'Q136-PAYMENT FOR FUEL OIL-HOME

'Q137-PIPE GAS AVAILABLE IN AREA'

'Q138-ELECTRICITY BILLED ON BUDGET'

'Q139-NATURAI GAS BILIED ON BUDGET'

'QO-1-TEMPERATURE OF HOT WATER'

'Q0-2-HOT WATER USED IN ILAST HOUR'

'Q0-3-USED HOT WTR IN IST HR-WASH

'QO-3-USED HOT WTR IN LST HR-WASH

'Q0-3-USED HOT WTR IN LST HR-BATHING'

'Q0-3-USED HOT WTR IN IST HR-OTHER'

'Q0-5-\# OF FLOORS USED AS LIVING

'Q0-6-\# OF FLOORS USED BELOW GROUND'

'Q0-7-\# OF FLOORS USED PART BELOW

'Q0-8-FLOOR THIS UNIT IS LOCATED'

'Q0-9-TYPE OF MATERIAL OUTSIDE-WOOD'

'Q0-9-TYPE OF MATERIAI OUTSIDE-BRICK' 
CONCRETE '

STUCCO'

SIDE'

SIDE '

SIDE
VAR4 14 VAR4 15

VAR4 16

VAR417

VAR4 18

VAR4 19

VAR420

VAR42I

CDIO

ID10

VAR422

VAR423

VAR424

VAR425

VAR426

VAR427

VAR428

VAR429

VAR430

VAR431

VAR432

VAR433

VAR434

VAR435

VAR4 36

VAR437

VAR4 38

VAR439

VAR440

VAR441

VAR4 42

VAR443

CDII

IDII

VAR444

VAR445

VAR446

VAR447

VAR4 48

VAR449

VAR450

VAR45I

VAR452

VAR453

VAR454

VAR455

VAR456
'Q0-9-TYPE OF MATERIAL OUTSIDE-STONE'

'Q0-9-TYPE OF MATERIAL OUTSIDE-

'Q0-9-TYPE OF MATERIAL OUTSIDE-

'Q0-9-TYPE OF MATERIAL OUTSIDE-ALUM

'Q0-9-TYPE OF MATERIAL OUTSIDE-STEEL

'Q0-9-TYPE OF MATERIAI OUTSIDE-COMP

'Q0-9-TYPE OF MATERIAL OUTSIDE-GIASS' 'Q0-9-TYPE OF MATERIAI OUTSIDE-OTHER'

- CARD NUMBER 10'

'SERIAL CASE IDIO'

'QB-BASEMENT TYPE'

'QOB-BASE UNIT A-LENGTH'

' OOB-BASE UNIT A-WIDTH'

'QOB-BASE UNIT B-LENGTH'

'QOB-BASE UNIT B-WIDTH'

'QOB-BASE UNIT C-LENGTH'

'QOB-BASE UNIT C-WIDTH'

'QOB-BASE UNIT D-IENGTH'

'QOB-BASE UNIT D-WIDTH'

'QOB-BASE UNIT X'

'QOB-NUMBER OF UNITS - BASEMENT'

'QOB-FIRST FLOOR TYPE'

'QOB-FIRST UNIT A-LENGTH'

'QOB-FIRST UNIT A-WIDTH'

'QOB-FIRST UNIT B-LENGTH'

'QOB-FIRST UNIT B-WIDTH'

'QOB-FIRST UNIT C-IENGTH'

'QOB-FIRST UNIT C-WIDTH'

'QOB-FIRST UNIT D-TENGTH'

'QOB-FIRST UNIT D-WIDTH'

'QOB-FIRST UNIT X'

'QOB-\# OF UNITS'

- CARD NUMBER il'

'SERIAI CASE IDII'

'QOB-SECOND FLOOR TYPE'

'QOB-SECOND FLOOR ATTIC'

'QOB-SECOND UNIT A-LENGTH'

'QOB-SECOND UNIT A-WIDTH'

'QOB-SECOND UNIT B-IENGTH'

'QOB-SECOND UNIT B-WIDTH'

'QOB-SECOND UNIT C-LENGTH'

'QOB-SECOND UNIT C-WIDTH'

'QOB-SECOND UNIT D-LENGTH'

'QOB-SECOND UNIT D-WIDTH'

'QOB-SECOND UNIT X'

'QOB-NUMBER OF UNITS - SECOND FLOOR'

'QOB-THIRD FLOOR TYPE' 


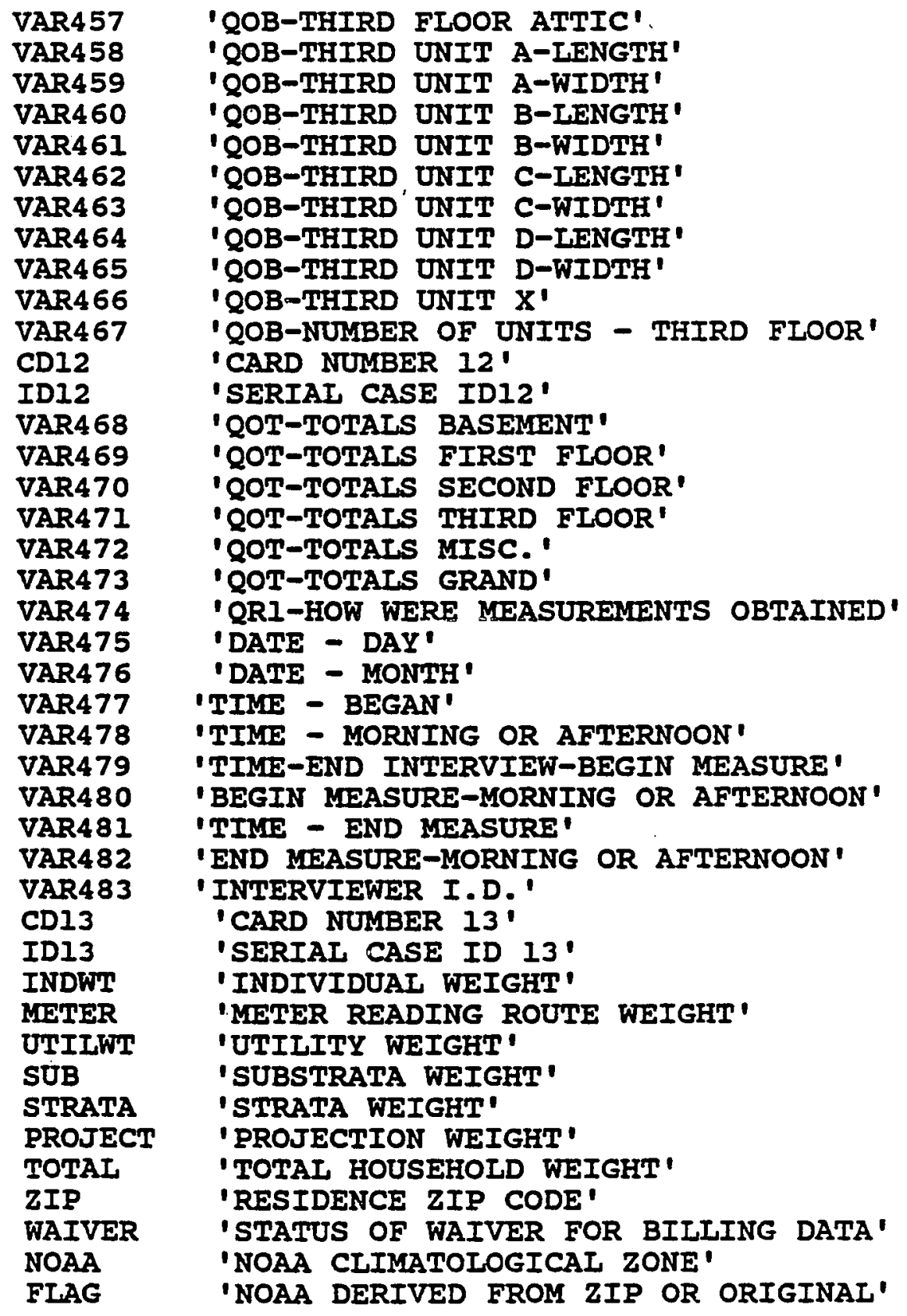




\section{APPENDIX V}

\section{A SAMPLE OUTPUT FOR THE INFORMATION THEORETIC PROGRAM, SYSENT}

This appendix presents the output for SYSENT, the program developed in order to perform the information theoretic calculations. The input consisted of cell frequencies for a contingency table of the six segmentation variables used in this study. These variables were GENATT, ENRATT, PERCEPT, AWARE, BEHAVE, and KWHUSE. The information theoretic analyses in Phase I and parts (a) and (b) of Phase II were performed using this output.

The information theoretic analyses for part (c) of Phase II were performed via a similar output, using a different contingency table. The variables for that contingency table consisted of KWHUSE, the distinguished segmentation base, and CLIMGEO, TYPDWEL, RENTOWN, DEMOG, and INSUL. 


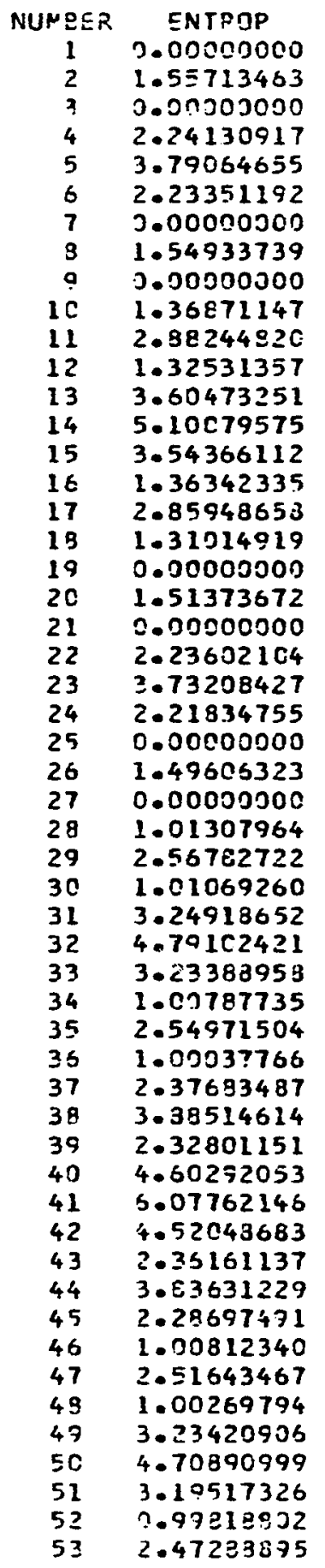

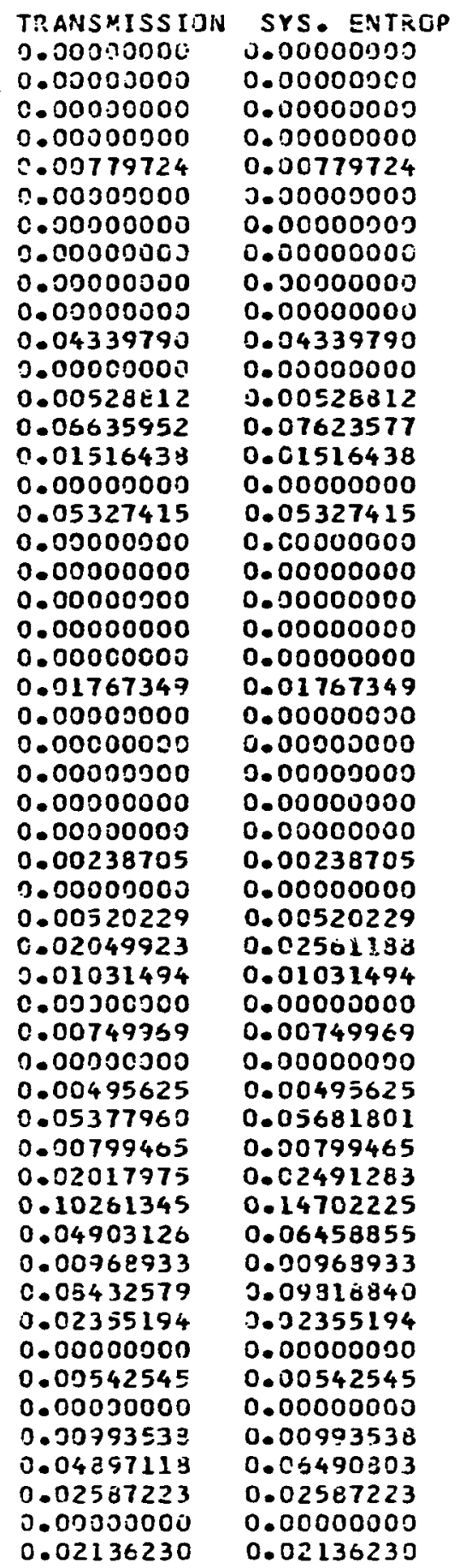

\begin{tabular}{|c|c|c|c|c|c|c|}
\hline \multicolumn{4}{|c|}{ O=ASSNT. } & \multicolumn{2}{|c|}{$I=P R E S}$, & $2=G$ IVEN \\
\hline ) & 0 & 0 & 0 & 0 & 0 & \\
\hline 1 & I & 0 & 0 & 0 & 0 & \\
\hline 2 & 0 & 0 & 0 & 0 & 0 & \\
\hline 0 & $\mathbf{1}$ & 0 & 0 & 0 & 0 & \\
\hline 1 & 1 & 0 & 0 & 0 & 0 & \\
\hline 2 & 1 & 0 & 0 & 0 & 0 & \\
\hline 0 & 2 & 0 & 0 & 0 & 0 & \\
\hline 1 & 2 & 0 & 0 & 0 & c & \\
\hline 2 & 2 & 0 & 0 & 0 & 0 & \\
\hline 0 & 0 & 1 & 0 & 0 & 0 & \\
\hline $\mathbf{L}$ & 0 & 1 & 0 & 0 & 0 & \\
\hline 2 & 9 & 1 & 0 & 0 & 0 & \\
\hline 0 & 1 & 1 & 0 & 0 & 0 & \\
\hline 1 & 1 & 1 & 0 & 0 & 0 & \\
\hline 2 & 1 & 1 & 0 & 0 & 0 & \\
\hline 0 & 2 & 1 & 0 & 0 & 0 & \\
\hline 1 & 2 & 1 & 0 & 0 & 0 & \\
\hline 2 & 2 & 1 & 0 & 0 & 0 & \\
\hline 0 & 0 & 2 & 0 & 0 & 0 & \\
\hline 1 & 0 & 2 & 0 & 0 & 0 & \\
\hline 2 & 0 & 2 & 0 & 0 & 0 & \\
\hline 0 & $\mathbf{l}$ & 2 & 0 & 0 & 0 & \\
\hline 1 & $\mathbf{1}$ & 2 & 0 & 0 & 0 & \\
\hline 2 & 1 & 2 & 0 & 0 & 0 & \\
\hline 0 & 2 & 2 & 0 & c & 0 & \\
\hline 1 & 2 & 2 & 0 & 0 & 0 & \\
\hline 2 & 2 & 2 & 0 & 0 & 0 & \\
\hline 0 & 0 & 0 & 1 & 0 & 0 & \\
\hline 1 & 0 & 0 & 1 & 0 & 0 & \\
\hline 2 & 0 & 0 & 1 & 0 & 0 & \\
\hline 0 & I & 0 & 1 & 0 & 0 & \\
\hline 1 & 1 & 0 & $\vec{i}$ & 0 & 0 & \\
\hline 2 & 1 & 0 & 1 & 0 & 0 & \\
\hline 0 & 2 & 0 & 1 & 0 & 0 & \\
\hline 1 & 2 & 0 & 1 & 0 & 0 & \\
\hline 2 & 2 & 0 & 1 & 0 & 0 & \\
\hline 0 & c & 1 & 1 & 0 & 0 & \\
\hline 1 & 0 & $i$ & $i$ & 0 & 0 & \\
\hline 2 & 0 & 1 & 1 & 0 & 0 & \\
\hline 0 & 1 & 1 & 1 & 0 & 0 & \\
\hline 1 & 1 & 1 & 1 & 0 & 0 & \\
\hline 2 & 1 & 1 & 1 & 0 & 0 & \\
\hline 0 & 2 & 1 & 1 & 0 & 0 & \\
\hline 1 & 2 & 1 & 1 & 0 & 0 & \\
\hline 2 & 2 & 1 & 2 & 0 & 0 & \\
\hline 0 & 0 & 2 & 1 & 0 & 0 & \\
\hline 1 & 0 & 2 & 1 & 0 & 0 & \\
\hline 2 & o & 2 & 1 & 0. & 0 & \\
\hline 0 & 1 & 2 & 1 & 0 & 0 & \\
\hline 1 & 1 & 2 & 1 & 0 & c & \\
\hline 2 & 1 & 2 & 1 & 0 & 0 & \\
\hline 0 & 2 & 2 & 1 & 0 & 0 & \\
\hline 1 & 2 & 2 & 1 & 0 & 0 & \\
\hline
\end{tabular}




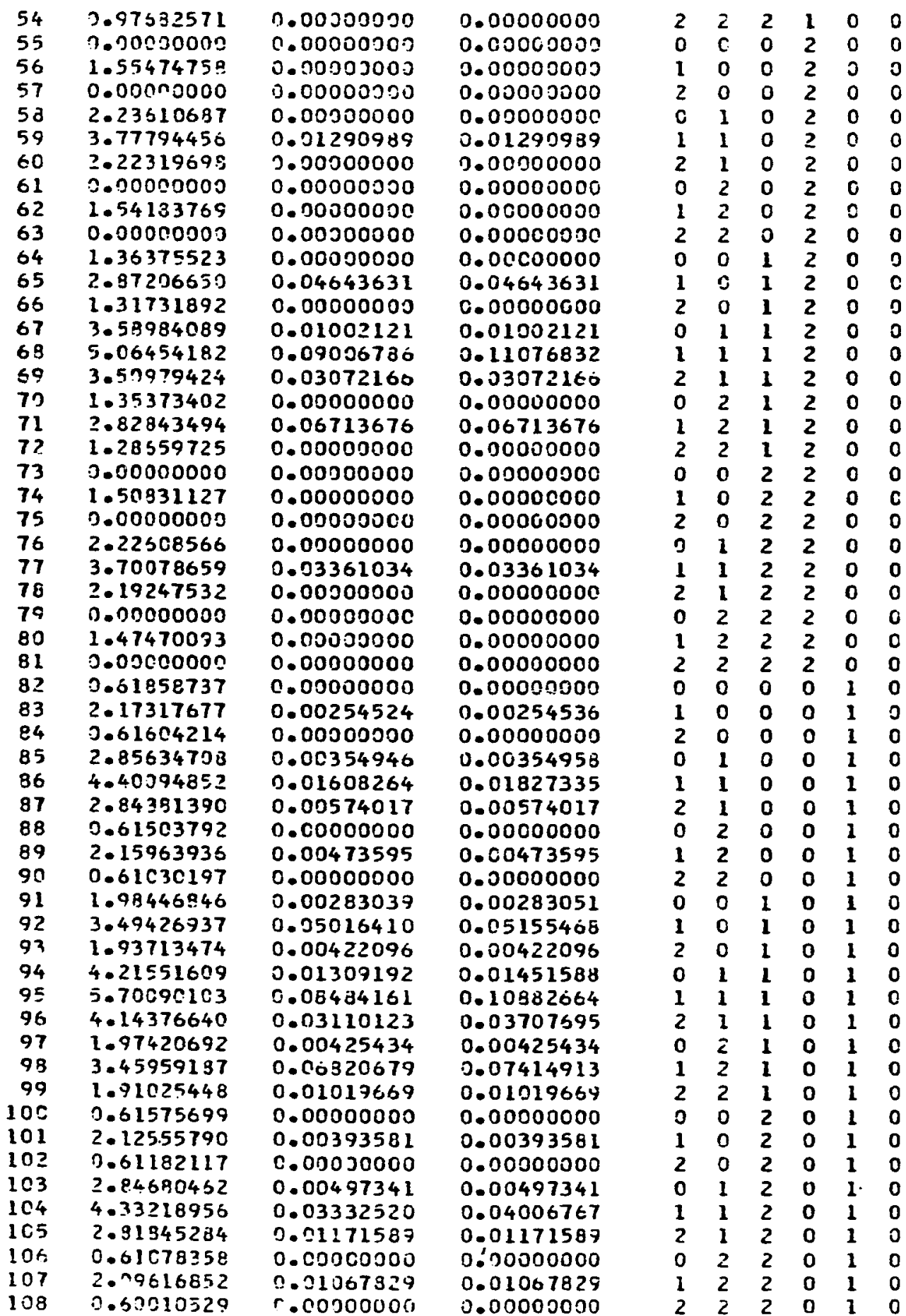


0.39350493 0.39906299 0.39413071 0.40194976 0.42664039 0.41391087 0.39319801 0.41009140 0.39785575 0.39994228 0.45516574 0.40683550 0.42413604 0.52466381 0.46953639 0.41009617 0.50282669 0.43731689 0.39215565 0.40398121 0.39461794 0.41206129 0.46819115 0.44115639 0.39615250 0.43560836 0.40356827 0.00000000 0.00317097 0.00000000 0.00324249 $0.025546 \mathrm{C7}$ 0.00746522 0.00000000 0.00939367 0.00000000 0.00148106 0.05431747 0.00471020 0.02047253

0.11361324 0.75009008 0.00720832 0.09243955 0.01590919 0.00000000 0.00540011 0.00000000 0.00897020 0.06067467 c. 02066422 0.00060000 $0.01809400^{\circ}$ 0.00000000 0.00503000
0.39350498 0.39968572 0.3941307 : 0.40164280 0.44232655

0.41763592

0.39319801

0.41474915

0.39735576

0.39859295

0.46254826

0.40732479

0.43724442

0.60525799

0.50473022

0.41305065

0.53575993

0.44302940

0.39215565

0.40644550

0.39461994

0.41505814

0.50723457

0.45010471

0.39615250

0.44302464

0.40356827

0.00000000

0.00317097

0.00000000

0.00324249

0.03176880

0.00946522

0.00000000

0.00939369

0.00000000

0.00148100

0.05754662

0.00471020

0.02620029

0.16543579

0.06729507

0.00720882

0.10114002

0.01590919

0.00000000

0.00640011

0.00000000

0.00697020

0.07236862

0.02066422

0.00000006

0.01809406

0.00000000

0.00000050

$\begin{array}{llllll}0 & 0 & 0 & 1 & 1 & 0 \\ 1 & 0 & 0 & 1 & 1 & 0 \\ 2 & 0 & 0 & 1 & 1 & 0 \\ 0 & 1 & 0 & 1 & 1 & 0 \\ 1 & 1 & 0 & 1 & 1 & 0 \\ 2 & 1 & 0 & 1 & 1 & 0 \\ 0 & 2 & 0 & 1 & 1 & 0 \\ 1 & 2 & 0 & 1 & 1 & 0 \\ 2 & 2 & 0 & 1 & 1 & 0 \\ 0 & 0 & 1 & 1 & 1 & 0 \\ 1 & 0 & 1 & 1 & 1 & 0 \\ 2 & 0 & 1 & 1 & 1 & 0 \\ 0 & 1 & 1 & 1 & 1 & 0 \\ 1 & 1 & 1 & 1 & 1 & 0 \\ 2 & 1 & 1 & 1 & 1 & 0 \\ 0 & 2 & 1 & 1 & 1 & 0 \\ 1 & 2 & 1 & 1 & 1 & 0 \\ 2 & 2 & 1 & 1 & 1 & 0 \\ 0 & 0 & 2 & 1 & 1 & 0 \\ 1 & 0 & 2 & 1 & 1 & 0 \\ 2 & 0 & 2 & 1 & 1 & 0 \\ 0 & 1 & 2 & 1 & 1 & 0 \\ 1 & 1 & 2 & 1 & 1 & 0 \\ 2 & 1 & 2 & 1 & 1 & 0 \\ 0 & 2 & 2 & 1 & 1 & 0 \\ 1 & 2 & 2 & 1 & 1 & 0 \\ 2 & 2 & 2 & 1 & 1 & 0 \\ 0 & 0 & 0 & 2 & 1 & 0 \\ 1 & 0 & 0 & 2 & 1 & 0 \\ 2 & 0 & 0 & 2 & 1 & 0 \\ 0 & 1 & 0 & 2 & 1 & 0 \\ 1 & 1 & 0 & 2 & 1 & 0 \\ 2 & 1 & 0 & 2 & 1 & 0 \\ 0 & 2 & 0 & 2 & 1 & 0 \\ 1 & 2 & 0 & 2 & 1 & 0 \\ 2 & 2 & 0 & 2 & 1 & 0 \\ 0 & 0 & 1 & 2 & 1 & 0 \\ 1 & 0 & 1 & 2 & 1 & 0 \\ 2 & 0 & 1 & 2 & 1 & 0 \\ 0 & 1 & 1 & 2 & 1 & 0 \\ 1 & 1 & 1 & 2 & 1 & 0 \\ 2 & 1 & 1 & 2 & 1 & 0 \\ 0 & 2 & 1 & 2 & 1 & 0 \\ 1 & 2 & 1 & 2 & 1 & 0 \\ 2 & 2 & 1 & 2 & 1 & 0 \\ 0 & 0 & 2 & 2 & 1 & 0 \\ 1 & 0 & 2 & 2 & 1 & 0 \\ 2 & 0 & 2 & 2 & 1 & 0 \\ 0 & 1 & 2 & 2 & 1 & 0 \\ 1 & 1 & 2 & 2 & 1 & 0 \\ 2 & 1 & 2 & 2 & 1 & 0 \\ 0 & 2 & 2 & 2 & 1 & 0 \\ 1 & 2 & 2 & 2 & 1 & 0 \\ 2 & 2 & 2 & 2 & 1 & 0 \\ 0 & 0 & 0 & 0 & 2 & 0 \\ & & & & & \end{array}$




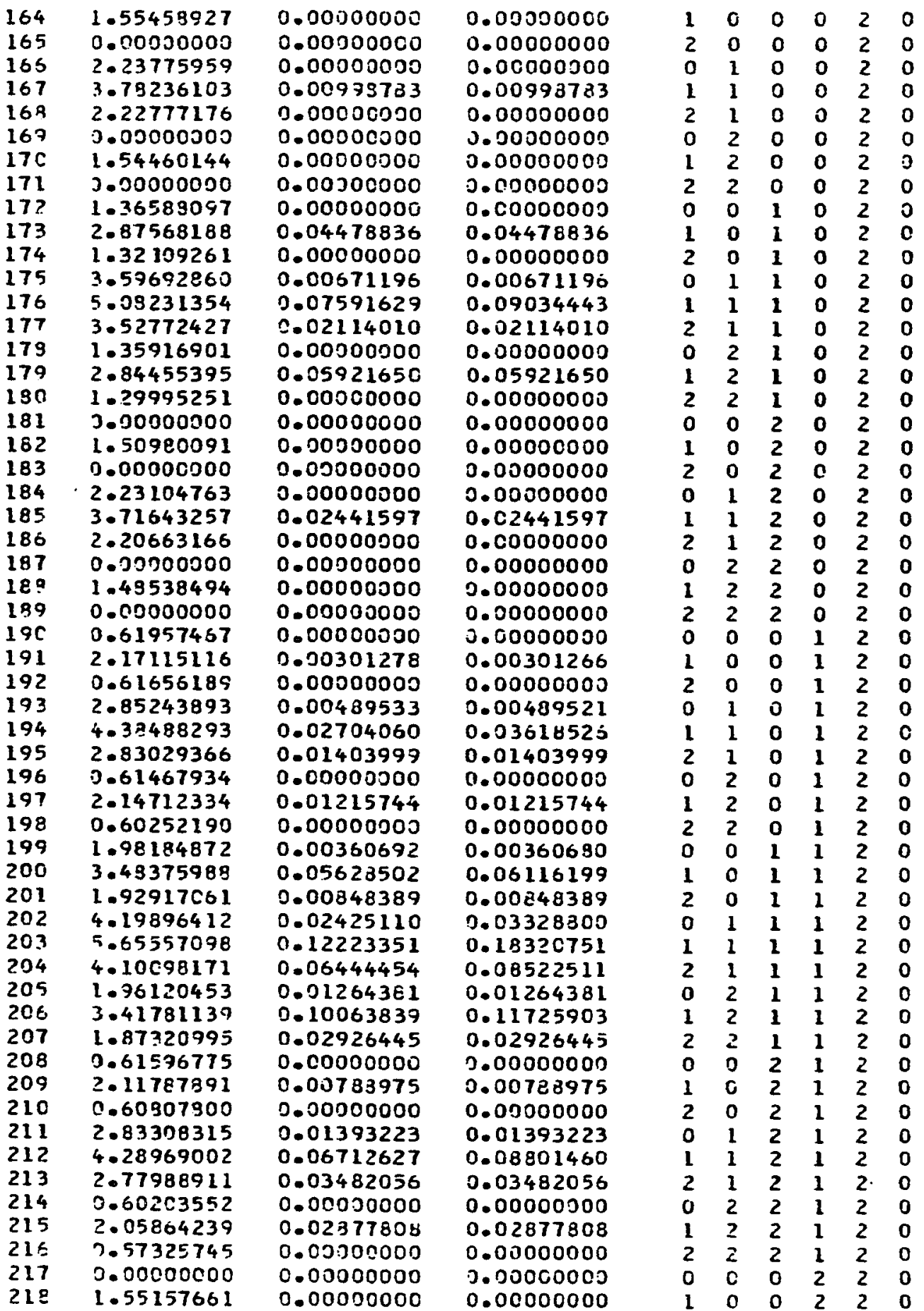


0.00000000

c. 00000000

0.01913261

0.00000000

0.00000003

0.00000000

0.00000000

0.00000000

0.04966545

0.00000200

0.01574898

0.11071873

0.04192060

0.00000000

0.07533714

0.00300000

0.00000000

0.00200000

0.00000000

0.00000000

0.04530430

0.00000000

0.00000000

0.00000000

0.00000000

0.00000000

0.00135517

0.00000000

0.00763607

0.02991357

0.02076626

0.00500000

0.01448536

0.00000000

0.00591373

0.05724716

0.01249409

0.02926636

0.12669563

0.07414532

0.01634216

0.10597420

0.03821468

0.00000000

0.00793552

0.00000000

0.01806450

0.07209587

0.04648685

0.00000000

0.03635788

0.00000000

0.00063038

0.01022053

0.00647831

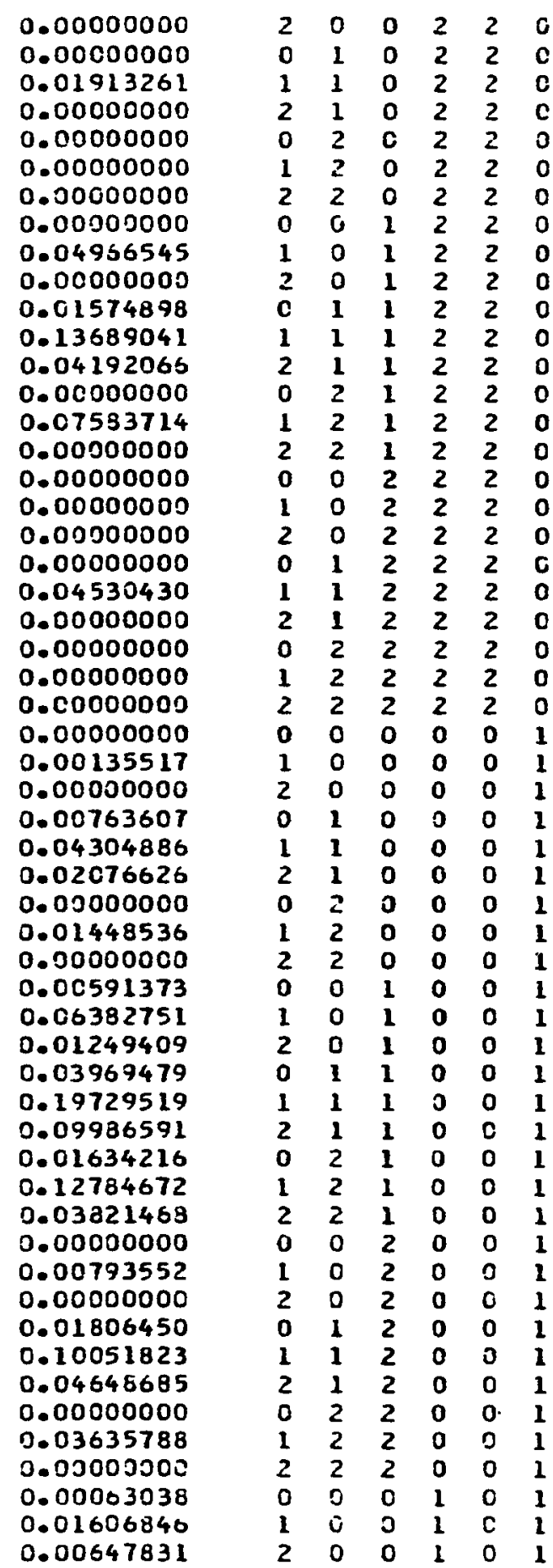




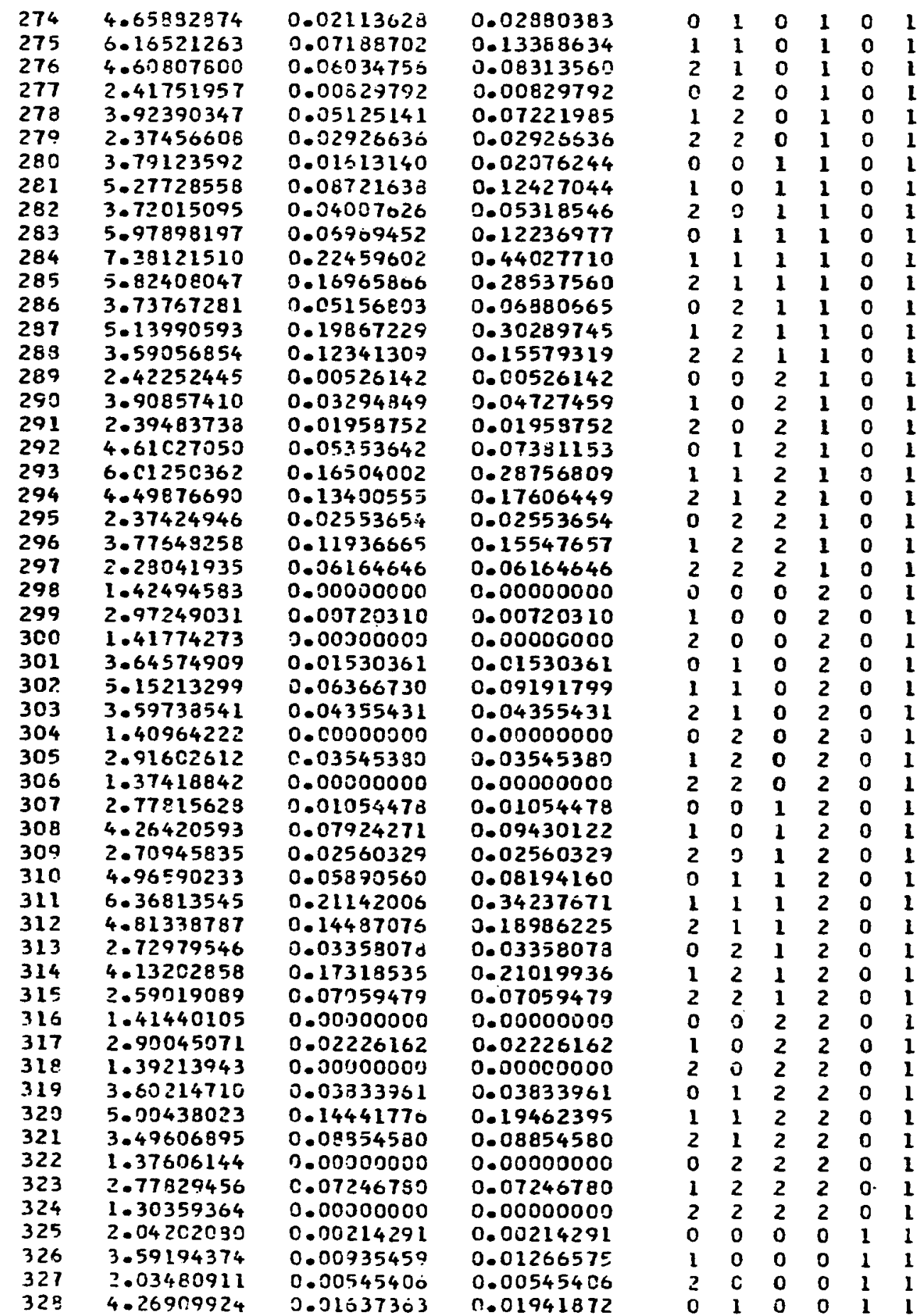


FILE= SHANON OUT
Al PORTLANO STATE UNIVERSITY VM/SP R4 03/27/87

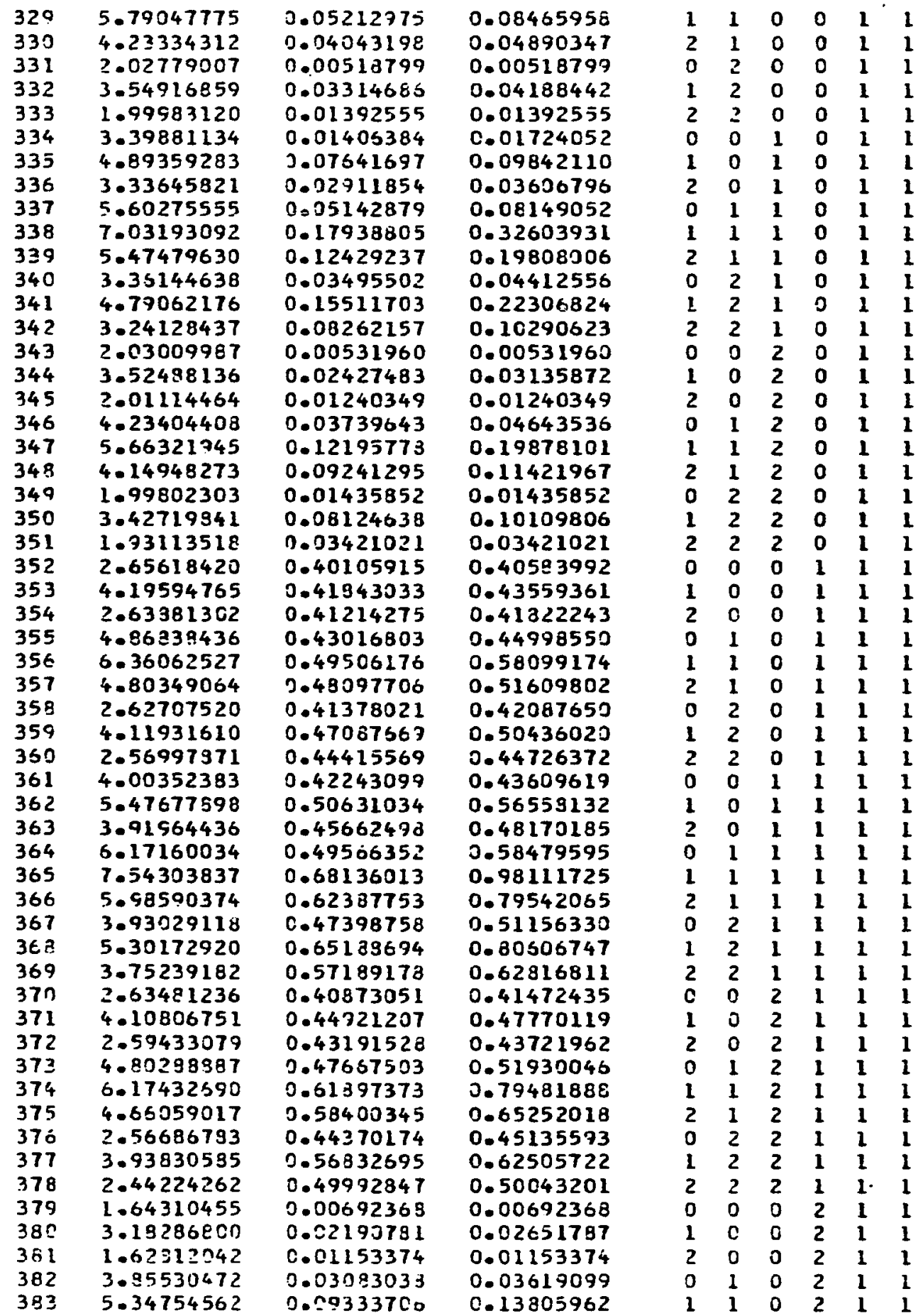


FILE: SHANCN CUT
A1 PORTLAND STATE UNIVERSITY VM/SP R4 03/27/87 
FILE: SHANON OUT
Al PORTLAND STATE UNIVERSITY VM/SP 24 03/27/37

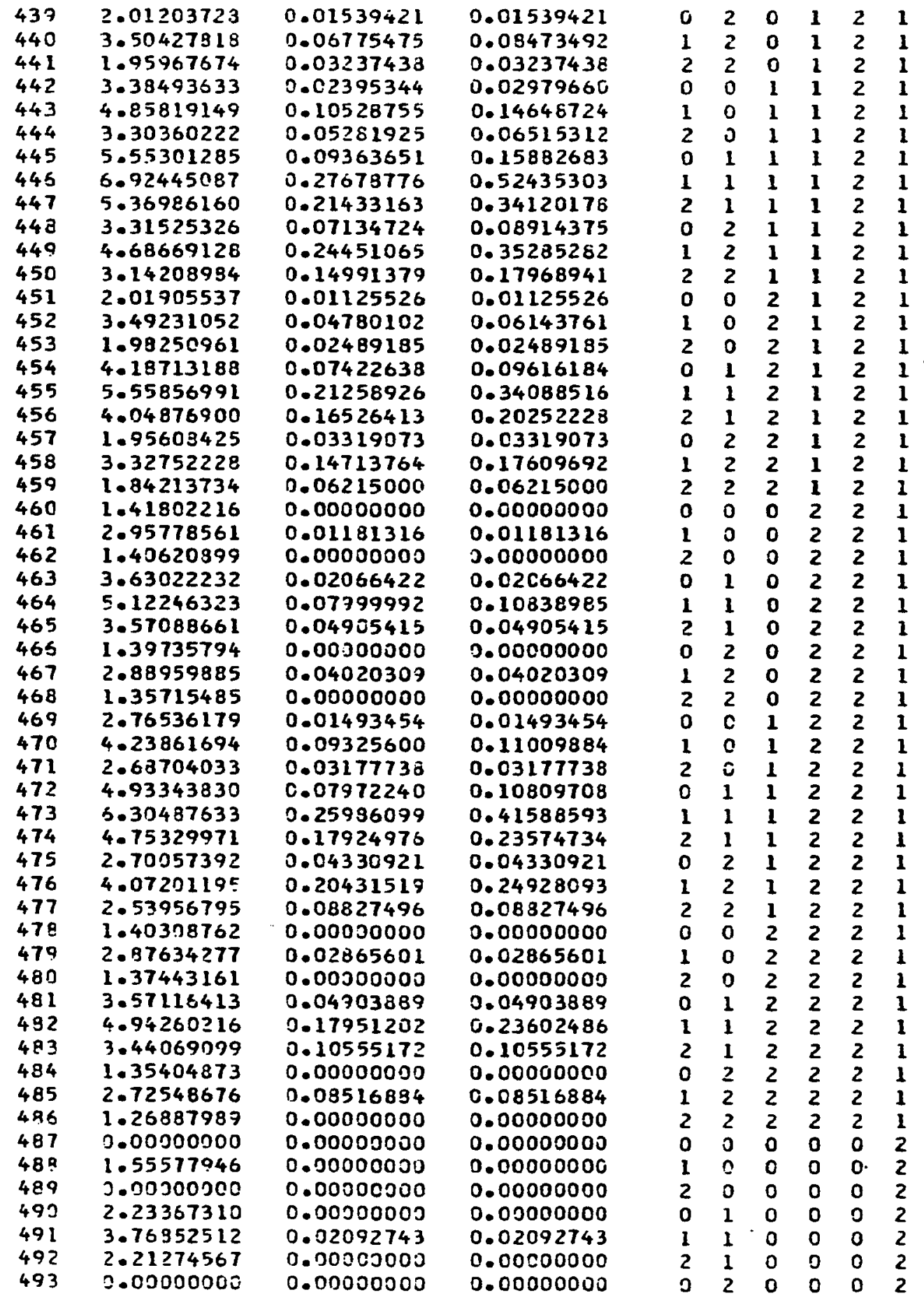


FILE: SHANON DUT
Al PORTLAND STATE UNIVERJITY VH/SP R4 $03 / 27 / 97$

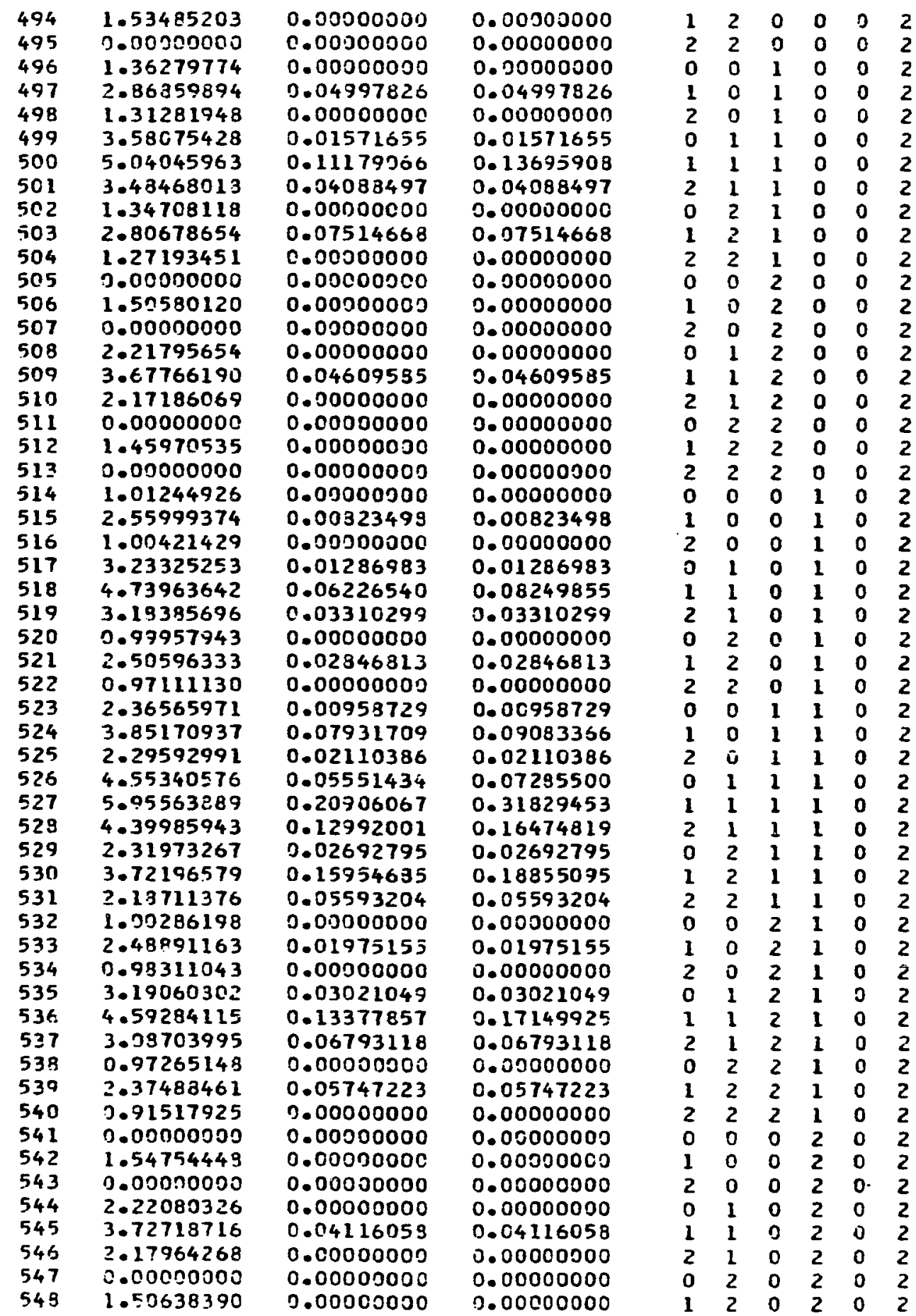


FILE: 3HANON DUT
AL PORTLAND STATE UNIVERSITY VM/SP R4 03/27/3T

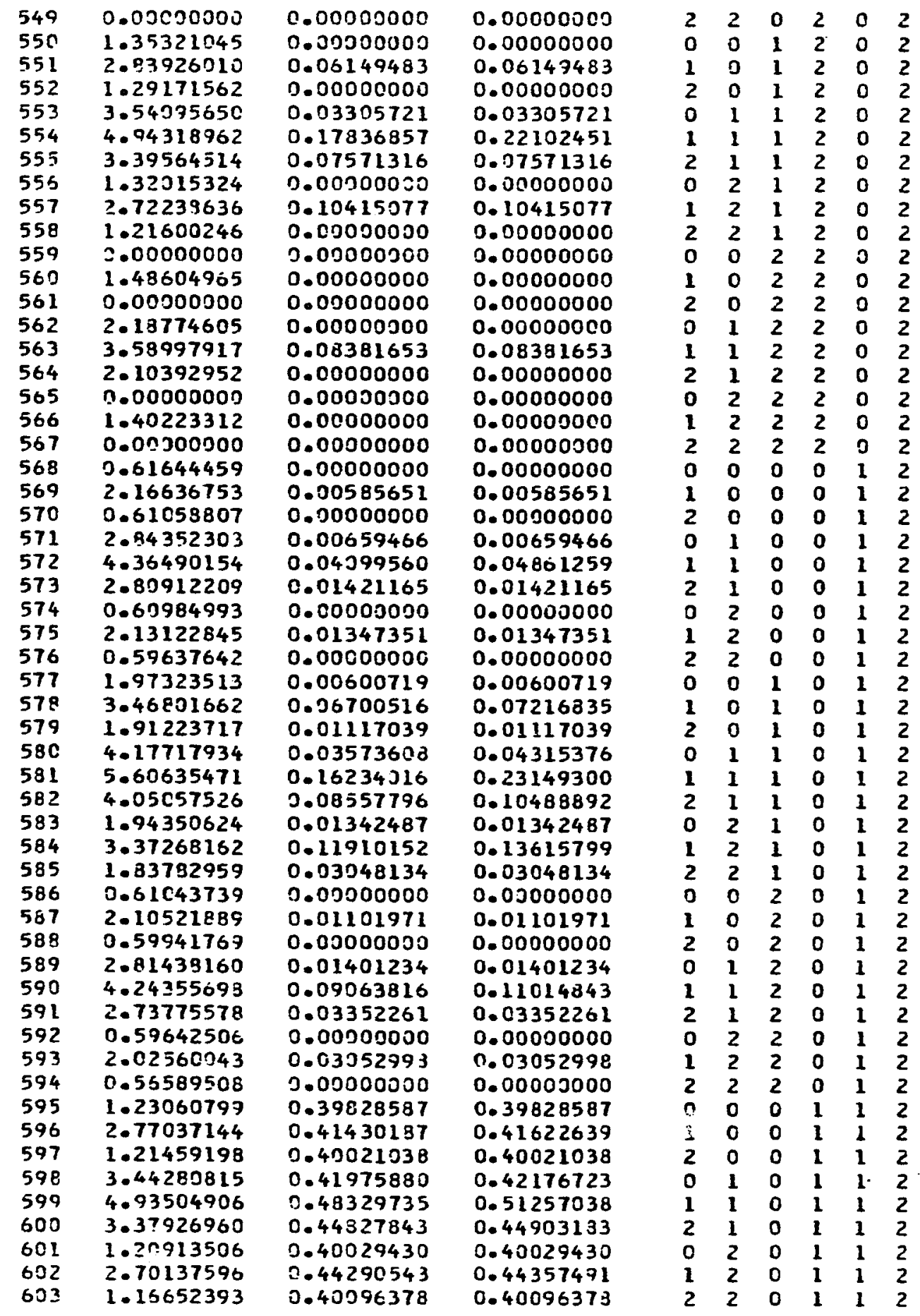


FILE: SHANON DUT
Al PORTLAHD STATE UNIVERSITY VM/SP R4 $03 / 27 / 37$

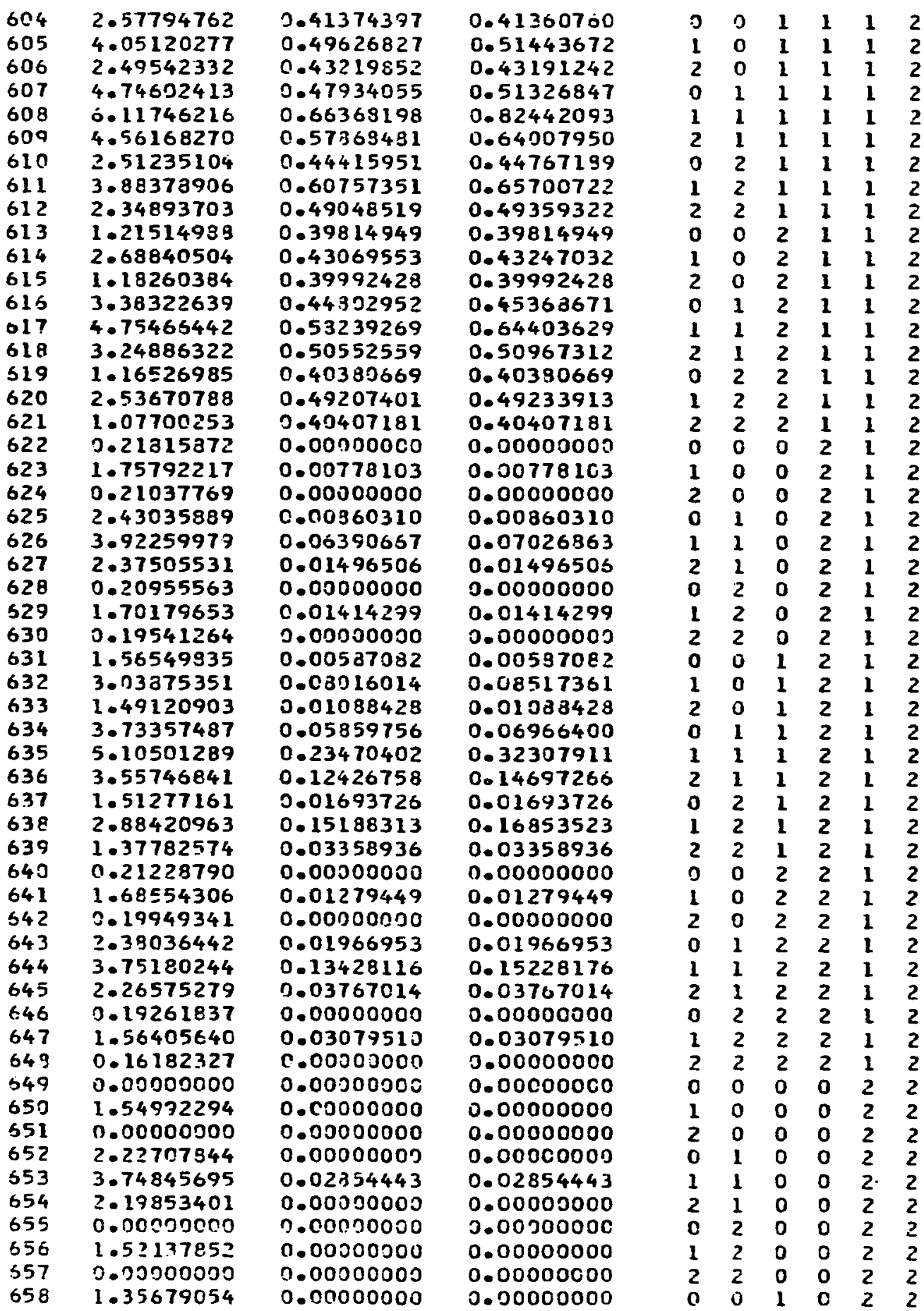




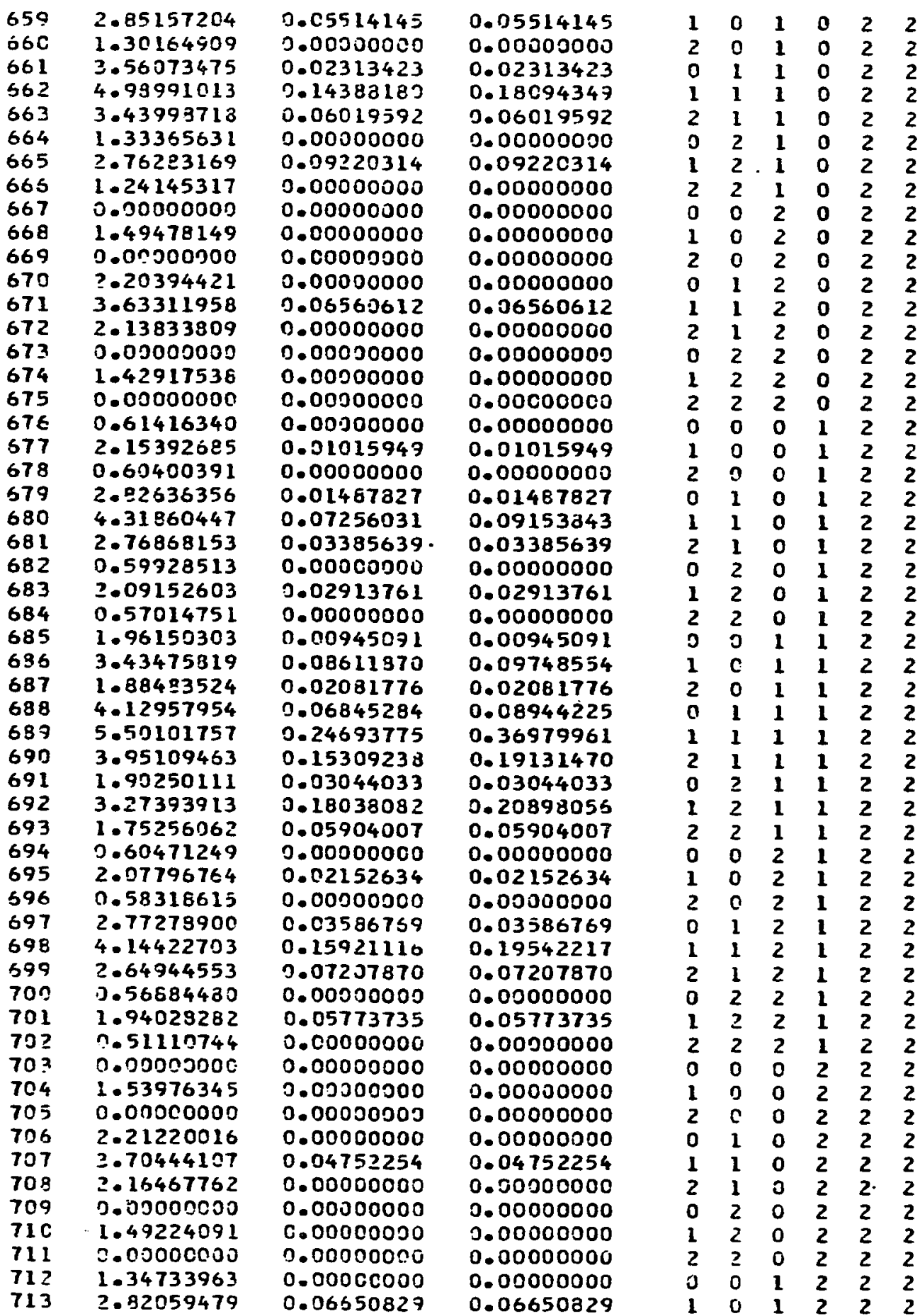




$\begin{array}{ll}714 & 1.28083134 \\ 715 & 3.51541615 \\ 716 & 4.88685417 \\ 717 & 3.34709072 \\ 718 & 1.30321593 \\ 717 & ? .67465401 \\ 720 & 1.18241310 \\ 721 & 0.0090000 \\ 722 & 1.47325516 \\ 723 & 0.00000000 \\ 724 & 2.16807652 \\ 725 & 3.53951454 \\ 726 & 2.06625938 \\ 727 & 0.00000000 \\ 728 & 1.37143903 \\ 729 & 0.00000000\end{array}$

$$
\begin{aligned}
& 0.00300000 \\
& 0.04412365 \\
& 0.21244907 \\
& 0.03841824 \\
& 0.00000000 \\
& 0.12080288 \\
& 0.00000000 \\
& 0.00000000 \\
& 0.00000000 \\
& 0.00000000 \\
& 0.00000000 \\
& 0.10181713 \\
& 0.00000000 \\
& 0.00000000 \\
& 0.00006000 \\
& 0.00000000
\end{aligned}
$$

0.00000000

0.04412365

0.26674366

0.09841824

0.00000000

0.12080288

0.00000000

0.00000000

0.00000000

0.00000000

0.00000000

0.10181713

0.00000000

0.00000000

0.00000000

0.00000000

$\begin{array}{llllll}2 & 0 & 1 & 2 & 2 & 2 \\ 0 & 1 & 1 & 2 & 2 & 2 \\ 1 & 1 & 1 & 2 & 2 & 2 \\ 2 & 1 & 1 & 2 & 2 & 2 \\ 0 & 2 & 1 & 2 & 2 & 2 \\ 1 & 2 & 1 & 2 & 2 & 2 \\ 2 & 2 & 1 & 2 & 2 & 2 \\ 0 & 0 & 2 & 2 & 2 & 2 \\ 1 & 0 & 2 & 2 & 2 & 2 \\ 2 & 0 & 2 & 2 & 2 & 2 \\ 0 & 1 & 2 & 2 & 2 & 2 \\ 1 & 1 & 2 & 2 & 2 & 2 \\ 2 & 1 & 2 & 2 & 2 & 2 \\ 0 & 2 & 2 & 2 & 2 & 2 \\ 1 & 2 & 2 & 2 & 2 & 2 \\ 2 & 2 & 2 & 2 & 2 & 2\end{array}$

